\title{
Ion and Surfactant Induced Wetting Transition
}

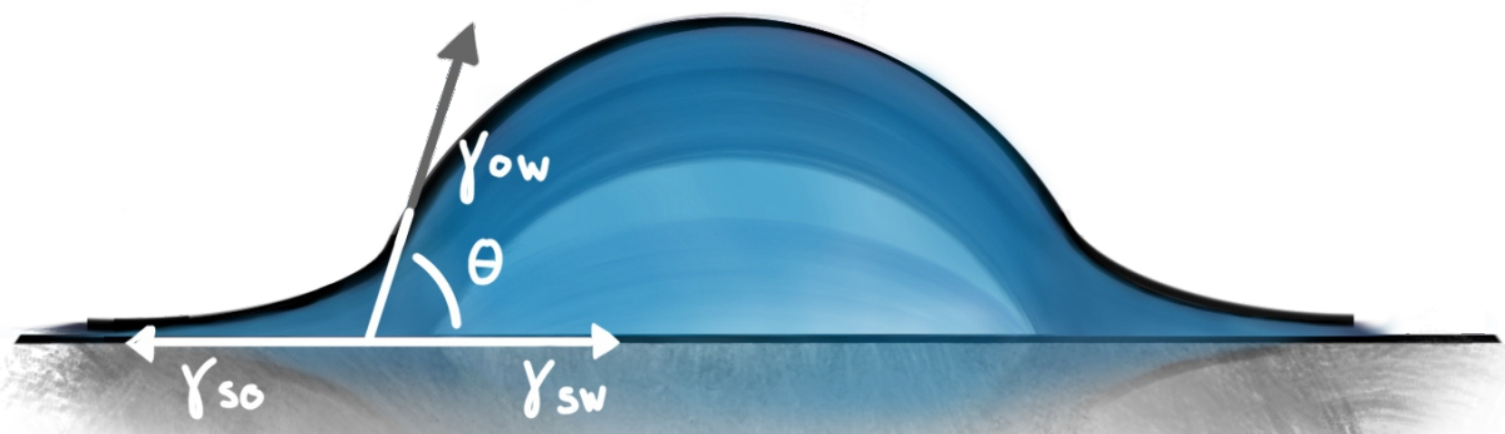

Bijoyendra Bera 


\section{Ion and Surfactant Induced Wetting Transition Bijoyendra Bera}


Graduation committee:

Prof. dr. ir. J.W.M. Hilgenkamp University of Twente, Chairman

Prof. dr. F. Mugele

University of Twente, Promotor

Prof. dr. M.A. Cohen-Stuart

Dr. M.H.G. Duits

University of Twente, Assistant promotor

University of Twente, Assistant promotor

Members:

Prof. A. Muggeridge

Prof. dr. R. van Roij

Prof. dr. D. Bonn

Prof. dr. ir. D.C. Nijmeijer

Prof. dr. ir. J. Huskens

Imperial College, London, UK

Utrecht University

University of Amsterdam

University of Twente

University of Twente
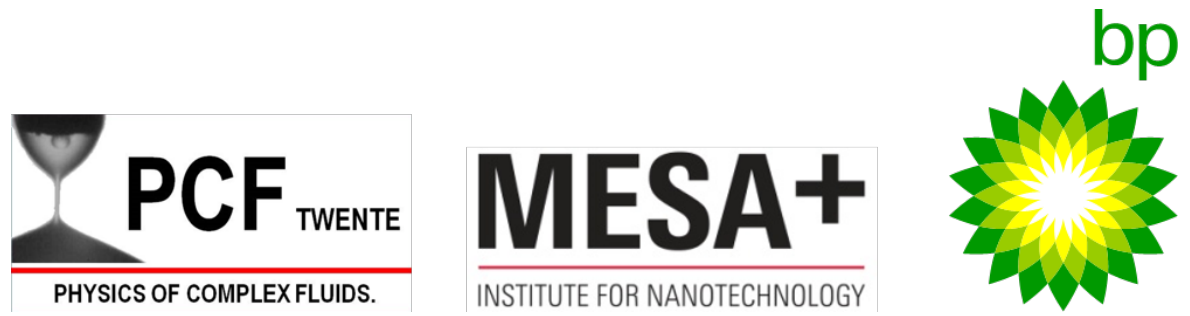

The research for this dissertation was carried out at:

Physics of Complex Fluids (PCF) Group

University of Twente

P.O. Box 217, NL - 7500 AE Enschede

DOI: $10.3990 / 1.9789036540490$

ISBN: 978-90-365-4049-0

Printed by GVO Drukkers en Vormgevers, Niels Bohrstraat 36, Ede

Cover fractal designed by Aram Klaassen and Arjen Pit

Copyright (C Bijoyendra Bera 2016

This work is supported by British Petroleum (BP plc.) within the Exploratory Research (ExploRe) program 


\title{
ION AND SURFACTANT INDUCED WETTING TRANSITION
}

\author{
PROEFSCHRIFT
}

ter verkrijging van

de graad van doctor aan de Universiteit Twente,

op gezag van de rector magnificus,

Prof. dr. H. Brinksma,

volgens besluit van het College voor Promoties,

in het openbaar te verdedigen

op vrijdag 12 februari 2016 om 16:45 uur

door

Bijoyendra Bera

geboren op 20 November 1987

te Jagacha, India 
Dit proefschrift is goedgekeurd door:

Prof. dr. F. Mugele (Promotor)

Prof. dr. M.A. Cohen-Stuart (Assistant promotor)

Dr. M.H.G. Duits (Assistant promotor) 
"To strive, to seek, to find, and not to yield"

- AlFRED, LORD TENNYSON 


\section{Summary}

One of the major challenges of modern society is the increasing demand for energy and conventional resources such as oil and natural gas are crucial for meeting this challenge. Hence it is of paramount importance to enhance the recovery process of these resources with a sustainable and environmentfriendly approach. The inherent pressure in an oil reservoir produces only a small fraction of the oil reserve (known as primary oil recovery), and even injecting water as a second phase (waterflooding) in the reservoir only increases the output to about half of the maximum reservoir capacity. A further incremental output is achieved via Enhanced Oil Recovery (EOR), which encompasses understanding the fundamental scientific processes during such an output enhancement. One of these processes is wettability alteration, where turning a oil-wet reservoir-rock into a water-wet surface releases oil droplets and ensures an incremental recovery.

This thesis focusses on wettability alteration, or wetting transition, on a solid phase from oil-wet to water-wet or vice versa. During oil recovery, the relative affinity between rock, water and oil governed by the ionic species plays a crucial role in such wetting transition. In Chapter 4, we investigate the competitive wetting of aqueous salt solution and an oil (decane) on mineral surfaces, where we observe a transition from a (nearly) complete wetting of monovalent salt $(\mathrm{NaCl}, \mathrm{KCl})$ solutions on mica, to a partial wetting state when divalent salt $\mathrm{CaCl}_{2}$ at high concentration and $\mathrm{pH}$ is present in the aqueous solution. We investigate the microscopic wetting films, where a thick to thin transition is observed as monovalent cations are replaced with divalent cations. In order to explain the phenomenon, we propose (and confirm with AFM) a mechanism, where adsorption of divalent cations at high concentration leads to a charge-reversal at usually negatively charged micawater interface, while the oil-water interface remains negatively charged.

In Chapter 5, we theoretically investigate the intermolecular interactions in our system, especially the electrostatics, which led to the wetting transi- 
tions. We calculate interaction energies including standard expressions from DLVO theory for van der Waals and electrostatic interactions and an empirical expression for short-range chemical forces. Furthermore, we extend the model to explore the consequences of charge regulation for the wettability of such systems. We address four generic shapes of the interface potential, corresponding to complete wetting, partial wetting, pseudo-partial wetting, and a metastable wetting film. We discuss the occurrence of these four scenarios in the parameter space that is given by the surface charge densities and the so-called (charge) regulation parameters of the two interfaces involved.

After investigating the electrostatic interactions extensively in the first part of the thesis, we focus on the more subtle influence of van der Waals and hydration interactions upon wetting in Chapter 6. A transition from (nearly) complete wetting to partial wetting is found in a mica-water-alkane system, if the cation-size is increased while keeping the valency same. In doing so, we present a hitherto unreported cationic adsorption/wettability sequence which resembles the famous Hofmeister series of protein crystallization. We identify the contributions of dispersion forces, surface hydration etc. to such an adsorption behavior or wetting transition, and discuss why charge-reversal is no longer required when all these factors play a role.

Surface active amphiphilic molecules (with hydrophilic as well as hydrophobic groups, also known as surfactants) in the crude oil are crucial in determining mineral wettability. Binding these surfactants to the surface of reservoir-rock is often aided by other ionic species. In Chapter 7, we investigate the effects of surface-active fatty acid in ambient oil on aqueous drops wetting mica and silica. A spontaneous increase in contact angle is observed (known as autophobing) in presence of divalent cations in the aqueous phase at a high $\mathrm{pH}$. We confirm that surfactants form a reaction-product with the divalent cations at the oil-water interface but is subsequently deposited on the solid by the retracting contact line. This leads to a gradient in the hydrophobicity of the substrate and a concomitant increase in contact angle. We develop an adsorption and transport based semi-quantitative model which assumes instantaneous mechanical equilibrium during the autophobing process, and a Langmuir-Blodgett type adsorption on the solid phase. We introduce two fitting parameters to capture the contact angle evolution, where one represents the relation between interfacial tension and coverage, and the other denotes the time-scale of autophobing. 


\section{Samenvatting}

Eén van de uitdagingen in onze samenleving ligt in de nog altijd toenemende vraag naar energie uit conventionele grondstoffen zoals olie en aardgas. Het is daarom noodzakelijk om de winning van deze brandstoffen te vergroten, en daarbij ook een zo duurzaam en millieuvriendelijke benadering te kiezen. De oorspronkelijke druk in een oliereservoir produceert slechts een klein deel van de totale oliereserve (primary oil recovery) en zelfs het inspuiten van (zee)water in het reservoir in een tweede fase (waterflooding) verhoogt de productie tot niet meer dan vijftig procent. Een verdere productieverhoging kan worden bereikt via Enhanced Oil Recovery (EOR). In deze fase van oliewinning wordt de samenstelling van de ingepompte vloeistof vaak beter afgestemd op de (verwachte) lokale situatie in het reservoir. Om de mate van succes in deze stap te verhogen, is een beter begrip van de fundamentele fysisch-chemische processen in het oliereservoir nodig. Een voorbeeld hiervan is de zogenaamde bevochtigingsovergang, waarbij een overgang van olie- naar een water-bevochtigd gesteente optreedt. Dit maakt het makkelijker om de aanwezige olie te verdrijven met de waterfase.

Dit proefschrift concentreert zich op bevochtigingsovergang op een vaste fase (het gesteente); van olie naar water-bevochtigd of andersom. Tijdens oliewinning, wordt de relatieve affiniteit van het gesteente met water en olie, bepaald door de verschillende ionen in het water; dit speelt een belangrijke rol in de overgang. In Hoofdstuk 4 onderzoeken wij de competitieve bevochtiging tussen zoutoplossingen en olie (decaan) op mineraaloppervlakken (bijv. mica). Wij ontdekken een overgang van een (bijna) complete bevochtiging met monovalente zoutoplossingen $(\mathrm{NaCl}, \mathrm{KCl})$, naar een gedeeltelijke bevochtiging met divalente zoutoplossing $\left(\mathrm{CaCl}_{2}\right)$ bij hoge concentratie en $\mathrm{pH}$. Wij bestuderen ook de microscopische bevochtigingslaag, waar een overgang van een dikke naar dunne laag wordt waargenomen als het kation wordt veranderd van mono- tot divalent kation. Om deze waarneming te verklaren, stellen wij een mechanisme voor (en bewijzen het met 
een atomic force microscope) waarbij de adsorptie van divalente kationen bij hoge concentratie en $\mathrm{pH}$ de lading van mica-water grensvlak omkeert (van negatief naar positief) terwijl het olie-water grensvlak negatief geladen blijft.

In Hoofdstuk $\mathbf{5}$ onderzoeken wij de interacties tussen de grensvlakken (vooral de elektrostatische) die de bevochtiginsovergang veroorzaken, door middel van een theoretisch model. Wij bereken de interactiepotentiaal inclusief elektrostatische en Van der Waals componenten (zoals in de DLVO theorie) en een empirisch vastgestelde uitdrukking voor chemische interacties. Wij breiden het model ook uit om de invloed van ladingsregulatie (aanpassing van de oppervlaktelading aan de afstand tussen de grensvlakken) te onderzoeken. Wij onderzoeken daarbij vier bevochtigingstoestanden: compleet, gedeeltelijk, pseudo-gedeeltelijk en metastabiel; en de bijbehorende potentiaalprofielen. Wij bediscussiëren deze toestanden in een parameteranalyse met oppervlakteladingsdichtheid en regulatieparameter.

$\mathrm{Na}$ het uitgebreide onderzoek naar de elektrostatische interacties in het eerste deel van het proefschrift, besteden wij aandacht aan de meer subtiele invloed van Van der Waals interacties en hydratatie op de bevochtiging in Hoofdstuk 6. Een overgang van (bijna) compleet naar gedeeltelijk bevochtiging wordt gevonden in een mica-water-alkaan systeem, indien een groter kation is gekozen zonder de kationvalentie te veranderen. Wij vinden een nieuwe rangschikking van kationen, analoog aan de klassieke Hofmeister serie voor de kristallisatie van eiwitten, maar nu voor de bevochtiging van waterdruppels op mica in olie. Wij herkennen onder andere de bijdragen van dispersie-interacties en oppervlaktehydratatie als de bron van deze adsorptieserie en bediscussiëren dat omkeren van oppervlaktelading niet strikt noodzakelijk is voor een bevochtigingsovergang.

Amfipolaire moleculen (met zowel hydrofiele als hydrofobe groepen, ook wel surfactants genoemd), in de aardolie spelen een belangrijke rol in de bevochtiging van oliehoudend gesteente. Hun adsorptie aan het gesteente wordt daarbij mede gecontroleerd door de aanwezigheid van specifieke ionen. In Hoofdstuk 7 onderzoeken wij de invloed van zowel de ionsamenstelling in het water, als van toegevoegd vetzuur in de olie, op de bevochtiging van twee mineraal substraten (mica en silica). Een spontane verhoging van de contacthoek (autophobing) wordt gevonden als de waterdruppels een voldoende hoge $\mathrm{pH}$ hebben en divalente kationen bevatten. Wij bevestigen dat de surfactants aan het olie-water grensvlak worden gevormd en ver- 
volgens neerslaan op de vaste fase via de (terugtrekkende) contactlijn. Dit leidt tot een gradient in hydrofobiciteit op het substraat, en daarmee ook tot autophobing. Dit verschijnsel wordt verder gemodelleerd met een wiskundige beschrijving van de adsorptie en transport, daarbij aannemende dat de momentane contacthoek wordt bepaald door de bekende wet van Young, en dat de depositie van surfactant verloopt zoals bij Langmuir-Blodgett transfer. Wij introduceren twee fitparameters om de contacthoek evolutie vast te leggen: één vertegenwoordigt het verband tussen grensvlakspanning en bedekking, de andere de tijdschaal van autophobing. Met dit model is een semi-kwantitative beschrijving van de experimenten mogelijk. 



\section{Contents}

1 Introduction 1

1.1 Enhanced Oil Recovery . . . . . . . . . . . . . . . . . . . . . 2

1.1.1 Oil Reservoirs . . . . . . . . . . . . . . . . . . 2

1.1.2 Phases of Oil Recovery . . . . . . . . . . . . . . . . 4

1.1.3 Waterflooding . . . . . . . . . . . . . . 4

1.2 Low Salinity Waterflooding . . . . . . . . . . . . . 4

1.2.1 Scientific Challenges . . . . . . . . . . . . . . 5

1.2.2 Competitive Wettability . . . . . . . . . . . . 6

1.3 Thesis Scope . . . . . . . . . . . . . . . . . . . . . . . . . 8

1.4 Thesis Outline . . . . . . . . . . . . . . . . . . . . . . . . 10

2 Theoretical Background 17

2.1 Introduction . . . . . . . . . . . . . . . . 18

2.2 Theory of Capillarity . . . . . . . . . . . . . . . . . . 18

2.2.1 Surface Tension . . . . . . . . . . . . . . . . . . . . 19

2.2 .2 Laplace Pressure . . . . . . . . . . . . . . . 20

2.3 Wetting . . . . . . . . . . . . . . . . . 21

2.3 .1 Young's Contact Angle . . . . . . . . . . . . . . 22

2.3.2 Spreading Parameter . . . . . . . . . . . . . . . . 23

2.4 Adsorption and Wetting . . . . . . . . . . . . . . 23

2.5 Basics of Adsorption . . . . . . . . . . . . . . . . 26

2.5.1 Langmuir Adsorption . . . . . . . . . . . . . . 26

2.5.2 Adsorption and Surface Tension . . . . . . . . . . 28

2.5.3 Rate Determining Process . . . . . . . . . . . . . . . . 29

2.6 Wetting and Disjoining Pressure . . . . . . . . . . . 31

2.6.1 Electric Double Layer . . . . . . . . . . . . . . . . . 31

Debye Hückel (DH) Approximation . . . . . . . . . . . . 33

Grahame's Equation . . . . . . . . . . . . . . . . 34

DH Equation and Grahame . . . . . . . . . . . . . . . . 34

2.6.2 Van der Waals Contribution . . . . . . . . . . . . . . . 35

2.6.3 Solvation and Steric Contribution . . . . . . . . . . 35 
$2.7 \quad$ Nature of $\Phi(h)$ and $\Pi(h) \ldots \ldots \ldots \ldots$

2.8 Wetting Configurations . . . . . . . . . . . . . . . . . 39

2.8.1 Wetting Transition . . . . . . . . . . . . . . 41

2.9 Surfactants . . . . . . . . . . . . . . . . . . . 41

2.10 Concluding Remarks . . . . . . . . . . . . . . . . 42

3 Experimental Methods 45

3.1 Introduction . . . . . . . . . . . . . . . . 46

3.2 Contact Angle Goniometry _. . . . . . . . . . . . . 46

3.2.1 Precision of Goniometry Measurements . . . . . . . . 46

3.2 .2 Magnification . . . . . . . . . . . . . . . 48

3.2 .3 Illumination . . . . . . . . . . . . . . . . . . 48

3.2 .4 Baseline . . . . . . . . . . . . . . . . . 48

3.2.5 Spherical Cap Fit . . . . . . . . . . . . . . . 50

3.2 .6 Reproducibility . . . . . . . . . . . . . . . 51

3.2.7 Final Remarks . . . . . . . . . . . . . . . . . . . 52

3.3 Pendant Drop Measurements . . . . . . . . . . . . . . . . 53

3.4 Ellipsometry . . . . . . . . . . . . . . . . . . 54

3.5 Streaming Potential Measurements . . . . . . . . . . . . 55

3.6 Langmuir Blodgett Trough . . . . . . . . . . . . . . . . 57

4 Ion-induced Wetting Transition in Mineral/Water/Oil systems $\quad 61$

4.1 Introduction . . . . . . . . . . . . . . . 62

4.2 Experimental Methods . . . . . . . . . . . . . . . . . 63

4.2 .1 Materials . . . . . . . . . . . . . . . . 63

4.2.2 Optical Goniometry . . . . . . . . . . . . . 63

4.2 .3 Imaging Ellipsometry . . . . . . . . . . . . . . 64

4.2.4 Streaming Potential Measurements . . . . . . . . . . . . 65

4.2 .5 Atomic Force Microscopy . . . . . . . . . . . . . . . 65

4.3 Results . . . . . . . . . . . . . . . . . 66

4.3.1 Cation-valency Influenced Transition . . . . . . . . 66

4.4 Mechanistic Insight . . . . . . . . . . . . . . . . . . . . . 68

4.4.1 Molecularly Thin Film . . . . . . . . . . . . . . 71

4.4.2 Surface Charge Measurement . . . . . . . . . . . . . 74

4.5 Concluding Remarks . . . . . . . . . . . . . . . . . 78

5 Electrostatic Modelling of Ion Adsorption Induced Wetting Transition $\quad 85$

5.1 Introduction . . . . . . . . . . . . . . 86

5.2 Complexation Model . . . . . . . . . . . . . . . . . . . . 87 
5.3 Constant Charge Model . . . . . . . . . . . . . . . . . . . . 92

5.4 Linearized Charge Regulation Model . . . . . . . . . . . . . . . 94

5.4.1 Wetting Phase-Space Analysis . . . . . . . . . . . 99

5.4.2 Constant Charge and Constant Charge-like Interfaces $-\Delta_{1,2}<0 \ldots \ldots \ldots \ldots$. . . . . . . . . 100

5.4.3 Constant Potential and Constant Potential-like Interfaces $-\Delta_{1,2}>0 \ldots \ldots \ldots \ldots \ldots 10 \ldots \ldots$

5.4.4 Comparison of Two Approaches . . . . . . . . . . . 102

5.5 Concluding Remarks . . . . . . . . . . . . . . . . . 103

5.6 Appendix . . . . . . . . . . . . . . . . . . . . . 104

5.6.1 Non-dimensionalization of Charge Regulation Model . 104

5.6.2 Calculation of Electrostatic Potential . . . . . . . . . 105

5.6.3 Disjoining Pressure and Interaction Potential . . . . . 107

6 Cationic Hofmeister Series of Wettability in Mica-water-alkane Systems

6.1 Introduction . . . . . . . . . . . . . . . . . . . 112

6.2 Experimental Methods . . . . . . . . . . . . . . . . . . . . 114

6.2 .1 Materials . . . . . . . . . . . . . . . . . . . . . 114

6.2.2 Optical Goniometry . . . . . . . . . . . . . . . . . 114

6.2 .3 Atomic Force Microscopy . . . . . . . . . . . . . . 115

6.3 Results . . . . . . . . . . . . . . . . . . . . 115

6.3.1 Order of Monovalent Cations . . . . . . . . . . . . . 115

6.3.2 Order of Divalent Cations . . . . . . . . . . . . . . 117

6.3.3 Influence of Anion, Alkane and $\mathrm{pH}$. . . . . . . . 120

6.4 Mechanistic Insight . . . . . . . . . . . . . . . . . . . . 121

6.4.1 Ion-specific Adsorption . . . . . . . . . . . . . . 123

6.4 .2 Force Measurements . . . . . . . . . . . . . . 126

6.4 .3 Discussion . . . . . . . . . . . . . . . . . . 128

6.5 Concluding Remarks . . . . . . . . . . . . . . . . . . . . 131

7 Surfactant Induced Autophobing 139

7.1 Introduction . . . . . . . . . . . . . . . . . . . . . 140

7.2 Experimental Methods . . . . . . . . . . . . . . . . . 141

7.2.1 Chemicals and Materials . . . . . . . . . . . . . . . . . . 141

7.2.2 Optical Goniometry . . . . . . . . . . . . . . . . . 142

7.2.3 Atomic Force Microscopy . . . . . . . . . . . . . . . . . 142

7.3 Results . . . . . . . . . . . . . . . . . . . . 143

7.3.1 Autophobing . . . . . . . . . . . . . . . . 143 
7.3.2 Extent of Wettability Alteration . . . . . . . . . . . . . 144

7.3.3 Evolution of Contact Angle . . . . . . . . . . . . 146

7.3.4 Generality of the Phenomenon . . . . . . . . . . . 147

7.4 Autophobing Mechanism . . . . . . . . . . . . . . 148

7.4.1 Surfactant Adsorption . . . . . . . . . . . . . . . 148

7.4.2 Surfactant Deposition . . . . . . . . . . . . . . 150

7.5 Theoretical Model . . . . . . . . . . . . . . . . . . . . . . 152

7.5.1 Comparison of Experiment \& Model . . . . . . . . . . 156

7.5.2 Rate Determining Step . . . . . . . . . . . . . . 157

7.5.3 Completion of Autophobing . . . . . . . . . . . . 159

7.6 Autophobing for Saturated Drops . . . . . . . . . . . . . 160

7.7 Concluding Remarks . . . . . . . . . . . . . . . . . . . . . 161

7.8 Appendix I: Autophobing Extended Experiments . . . . . . 163

7.8.1 Various Surfactant Concentrations . . . . . . . . . . 163

7.8.2 Influence of Fatty Acid-Chain Length . . . . . . . . 164

7.8.3 Influence of Alkane-Chain Length . . . . . . . . . 164

7.8.4 Cationic Surfactant . . . . . . . . . . . . . . . 167

7.9 Appendix II: Calculation for Autophobing Modeling . . . . . 168

7.10 Appendix III: Wettability Alteration on Langmuir-Blodgett Lay-

ers . . . . . . . . . . . . . . . . . . 173

7.10 .1 Chemicals and Materials . . . . . . . . . . . . . . 173

7.10 .2 Langmuir-Blodgett Method . . . . . . . . . . . . . 174

7.10 .3 Dynamic Contact Angle Behavior . . . . . . . . . . 174

8 Conclusions and Outlook 181

8.1 Thesis Contributions . . . . . . . . . . . . . . . . . . . 181

8.2 Future Possibilities . . . . . . . . . . . . . . . . . . . 183

8.2.1 Lab-scale Low Salinity Waterflooding . . . . . . . . . 183

8.2.2 Wettability Alteration \& Microfluidics . . . . . . . . . 184

8.2.3 Wetting at Reservoir Temperature . . . . . . . . . . 184

8.2.4 Wetting at Reservoir Pressure . . . . . . . . . . . 185

$\begin{array}{ll}\text { Contributions } & 187\end{array}$

$\begin{array}{ll}\text { About the Author } & 189\end{array}$

$\begin{array}{ll}\text { Acknowledgements } & 191\end{array}$ 


\section{CHAPTER 1}

\section{Introduction}

\section{Abstract}

The ever-increasing demand for energy in our society calls for more efficient and sustainable recovery of fossil fuels. Enhanced Oil Recovery (EOR) deals with improving the recovery of oil from reservoirs. It encompasses not only the large-scale engineering enterprise, but also the fundamental understanding and discovery of the relevant physicochemical processes. The motivation for this thesis stems from this fundamental interest in EOR, more particularly from a specific method of EOR known as low salinity waterflooding. Conventional waterflooding involves flooding the oil reservoir with water (off-shore reservoirs with locally available brine) for improved oil recovery and in recent years, the influence of the composition of this injected aqueous phase on the incremental output of oil has drawn attention. Wettability of the reservoir-rock is a significant property influenced by low salinity waterflooding and is believed to determine the success of the production. Various processes governing the wettability for this specific example have been investigated and discussed over decades, albeit without universal agreement. This is because of the complex interplay of ions, polar components, rock or mineral surface constitution over multiple lengh-scales. We are interested in this specific parameter, wettability, in this thesis from an ion adsorption and interaction perspective. In this chapter, the broad motivation of the work is first introduced, and subsequently, the fundamental questions to be addressed are discussed. 


\subsection{Enhanced Oil Recovery}

The new century has seen an exponential upsurge in the demand for energyresources in order to keep pace with the industrial usage as well as with a city-centric lifestyle. Renewable energy resources have been identified as the long-term solution for such ever-increasing energy demands. But our society lacks the infrastructure for harnessing such a huge amount of energy from solar, wind or other renewable resources, which makes the per capita cost for production and distribution of renewable energy quite high. In developed countries, the process of switching to renewable energy resources is more robust, e.g., in 2015, 33\% of electrical energy in Germany was produced by renewables (the biggest source) with $18 \%$ wind resources and $8 \%$ photovoltaics. Nonetheless, conventional resources remain the major provider of energy in our society by far while presently, only $1 \%$ of all electric power in the world is generated by photovoltaic devices. The reserves for conventional fossil fuel resources, on the other hand, are plenty to meet the demand for another half a century. With its existing infrastructure, oil and natural gas are going to play a significant role in our search of a stable energy-economy. It is crucial to recover these resources in an efficient and environment-friendly manner while we slowly switch to a different energy environment. In this thesis, the main research questions are motivated by more efficient recovery methods of oil reserves, known as Enhanced Oil Recovery $[1,2]$. Before formulating the research questions, however, we will describe the oil reservoir and the scientific challenges of oil recovery in brief.

\subsubsection{Oil Reservoirs}

Oil is formed as a result of the influence of time and pressure on organic materials. Geological products containing organic materials from trees and animals are buried well underneath the earth-surface over a span of millions of years and slowly turned into crude oil or 'petroleum'. The word originates from a combination of latin 'petra' (rock) and 'oleum' (oil), implying oil available from rock. Figure 1.1 shows a schematic of a typical oil reservoir. The oil is formed in source rock, i.e., a layer which is strong enough to sustain such long influence of temperature and pressure. Once the crude oil is formed, it permeates to the porous bed of mineal rock structure known as reservoir rock. Oil-bearing reservoir rocks are a few kilometers in length and up to a few kilometers deep from the earth's surface, while the width varies from a few tens of meters to a few hundred meters. They can be found 


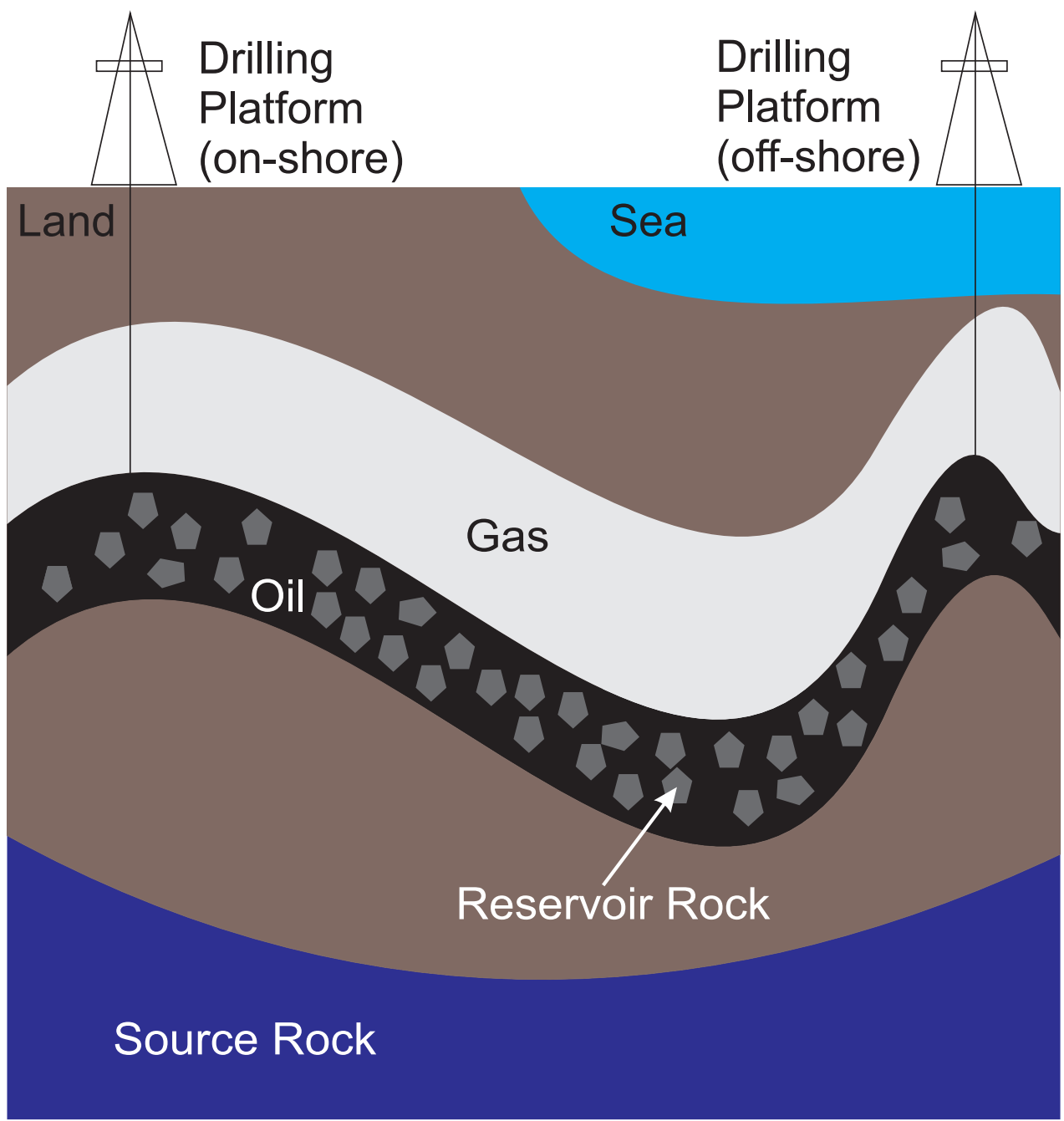

Figure 1.1: Schematic of an oil reservoir with on-shore and off-shore drilling opportunities. Various length scales are present together in the schematic, from kilometer scale of the entire reservoir to the $\mu \mathrm{m}$ scale of porous reservoir rock.

underneath land (on-shore reservoirs) or seas (off-shore). The mineral surfaces of reservoir-rock consist of silica, alumina, feldspar etc., since the reservoirrock is usually sandstone or carbonate. The porosity of the oil reservoir depends on the specific type of rock that is present and can vary from 10-35\% [3]. 


\subsubsection{Phases of Oil Recovery}

The primary phase of oil recovery involves drilling at a reservoir-site, where about $10 \%$ of the oil present is recovered by the inherent reservoir pressure. This stage is known as 'primary oil recovery'. This is followed by the 'secondary' phase of recovery, where a second fluid phase is introduced in the reservoir. For off-shore reservoirs, brine is used for this purpose, and the method is called waterflooding. This introduced phase maintains the pressure inside the reservoir, resulting in an increased output, often up to $50 \%$ of the total resource [1]. But even this number is quite low. Further methods involve improving the conditions for a secondary recovery method or introducing a gaseous phase or surfactants in the reservoir $[4,5,6]$, and are referred as 'tertiary oil recovery'. The total oil reserve of our planet Earth is estimated at 200 billion $\mathrm{m}^{3}$ and the chances of discovering a new oil reservoir are becoming smaller. Hence, even a fractional increase in the recovery percentage is extremely desirable. Among the various methods of oil recovery, in this thesis we are interested in 'Waterflooding'.

\subsubsection{Waterflooding}

During the last half a century, waterflooding has been the most used secondary oil recovery method. The popularity of water lies in the easy availability of it. A significant volume of water is injected in the reservoir and some of this water (produced alongside oil) is re-injected into the reservoir for increasing secondary oil recovery [7]. Usually, the phase introduced in the reservoir is seawater, which contains $\sim 500 \mathrm{mM} \mathrm{NaCl}, 50 \mathrm{mM} \mathrm{MgCl}_{2}, 10$ $\mathrm{mM} \mathrm{CaCl} 2$ and other salts in relatively lower quantities [8]. It has been observed in preceding years $[9,10]$ that reducing the salt content in brine yields more oil. This phenomenon is called low salinity waterflooding (LSW) and will be one of the central themes of our experimental investigation.

\subsection{Low Salinity Waterflooding}

In the past couple of decades, low salinity waterflooding has gained popularity because the method is able to increase the production of oil up to $25 \%$ in laboratory-scale waterflooding especially in sandstone rocks (Figure 1.2) $[9,10]$. Many mechanisms have been proposed to explain this phenomenon. Mobilization of fines (very small clay particles) [11] assumes that polar components of crude oil are readily adsorbed onto fines on the pore walls. Dur- 
ing low salinity flooding, these polar components are desorbed, leading to moving fines and along with it, the crude oil. The theory of cation bridging $[11,12,13]$ focusses on the abundance of multivalent cations in the brine solution (high-salinity) and their ability to turn the reservoir-rock oil-wet by enhancing the binding of polar oil molecules to the mineral surface. Other speculations such as the $p H$ effect, the lowering of interfacial tension [14] and particle-stabilized interfaces [15] have been investigated widely in this context. The $\mathrm{pH}$ effect implies an increase in $\mathrm{pH}$ due to carbonate dissolution leading to a decrease of oil-water interface tension, which in turn facilitates water-wettability of rock surface. Particle stabilized interfaces are another option, where clay particles are adsorbed onto the rock surface enhancing the oil wettability of rock. In spite of the experimental evidence partially supporting these various theories, consensus about one underlying mechanism of low salinity waterflooding is still missing.

\subsubsection{Scientific Challenges}

In order to delve deeper into oil recovery mechanisms, one has to appreciate the broad scope of the problem. The relevant physical and chemical phenomena occur over multiple length-scales, starting from macroscopic flow at the kilometer-scale of the entire reservoir, to the $\mathrm{mm}$-scale of fine migration, where the relative displacement of oil and water plays a crucial role. These incidents are, in turn, a result of pore-scale (a few hundred nm to micron-scale) phenomena such as capillarity. Finally, there is the molecular scale, where adsorption and molecular interactions result in incidents which might contribute to changes in wettability. We will focus mainly on these last two length-scales in this thesis, since we have identified wetting as the central theme of our work. It is important to underline the two principal factors affecting wetting on this scale: (i) geometry or structural properties of the reservoir and (ii) physicochemical phenomena. The geometry of the pore space is important because, wettability is inherently related to the heterogeneity of the substrate. In this case, the structural heterogeneity originates from the labyrinthine pore-space in the reservoir. Usually, the pore space is represented as a combination of discrete spherical pores connected by cylindrical constrictions or throats $[17,18]$. A large volume work has been dedicated to modeling the pore space of a reservoir-rock [19, 20,21,22], and modeling the effects on reservoir wettability [23]. On the other hand, not every aspect of wettability is dependent on the geometry, but rather is governed by a complicated interplay of ion interaction and adsorption, contributing to 


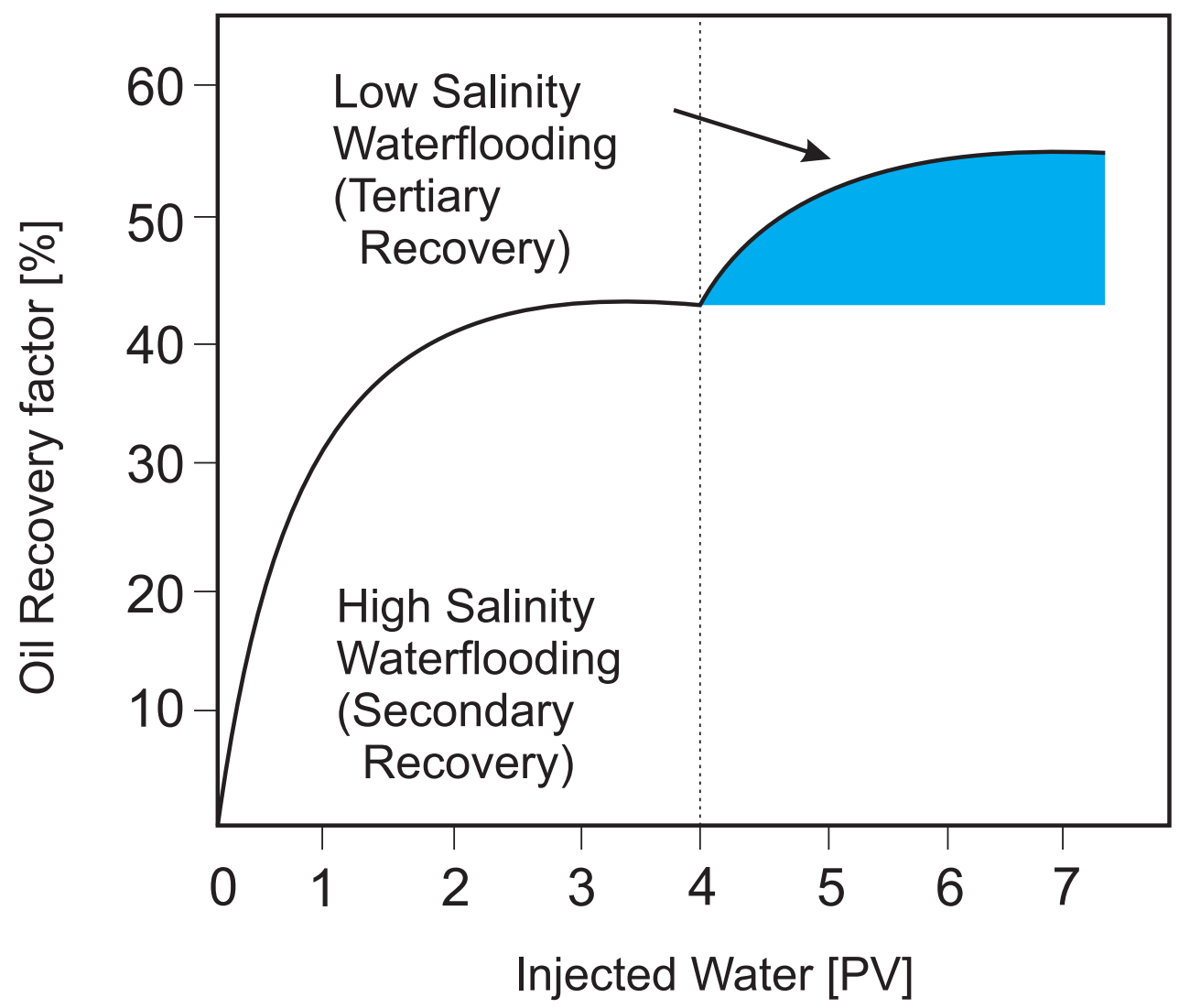

Figure 1.2: A standard representation of the succes of low salinity waterflooding through 5-10\% increase in the output. Figure adapted from [16].

the chemical heterogeneity of the substrate. We will dedicate our attention to these aspects in this thesis.

\subsubsection{Competitive Wettability}

Even though the possibilities about the governing process are diverse, and the implications of many physiochemical processes in an oil-reservoir are much debated, there is more solidarity among researchers about the effect of wettability change during this process. It is important to note here, that by the term 'wettability', we mean the physical picture of adhesion involving contact angle, and not the empirical measures of relative wetting often used in petroleum engineering. In order to observe an incremental oil produc- 
tion, the reservoir rock material has to turn water-wet from oil-wet [24, 25]. This invokes the question whether the water or the oil phase exhibits a preferential adhesion to the rock surface. If one of these two phases does have a stronger affinity to the rock surface, then the point of interest is: what is the contact angle for this phase and is there a way to release this phase by increasing the contact angle? This is a typical problem of wetting transition for a physical chemist, where individual interactions between wetting or non-wetting phases and the relevant molecules can be investigated in order to achieve such a feat. The basic concepts of wetting and how they are influenced by molecular interactions are discussed in standard textbooks or review articles [26, 27]. In the case of waterflooding, the basic concepts must be appended by special considerations for ion-ion correlation, charge effects, ion-specific effects, etc. The influence of charged species (on the rock surface or in the fluid phase i.e., oil and water) on wetting is related via the welldefined concept of disjoining pressure, but in a dynamic system such as an oil reservoir, the changes in surface charge influenced by the adsorption of multivalent ions and polar components must be taken into account as well. The advent of force measurement and atomic resolution imaging techniques using atomic force microscopy (AFM) provides hitherto unimaginable insight into adsorption and surface charge [28, 29, 30, 31]. Non-electrostatic effects, such as ion-specific effects, are also important to explain the aggregation of dissolved macromolecules (e.g., polar components in the oil), a phenomenon known as the 'Hofmeister Effect'. The effect is important since it determines the preferential adsorption of an ionic component onto a surface. Furthermore, such effects become extremely relevant in the presence of multi-component crude oil and clay particles.

Figure 1.3 depicts a schematic impression of the waterflooding process, where the injected water phase maintains the pressure in the pore-space for improved recovery. However, the goal is to achieve much more than a mechanical maintenance of pressure. We have highlighted a part of the rocksurface in Figure 1.3, where the relative wettability of the rock surface to water and oil dictates the retention/release of the adjacent oil drop. Hence, the various species present in the aqueous phase, the oil phase as well as the rock surface are all crucial for triggering the release of the oil. A set of complex interactions between these species are responsible for determining the switch from an oil-wet to a water-wet rock-surface. 


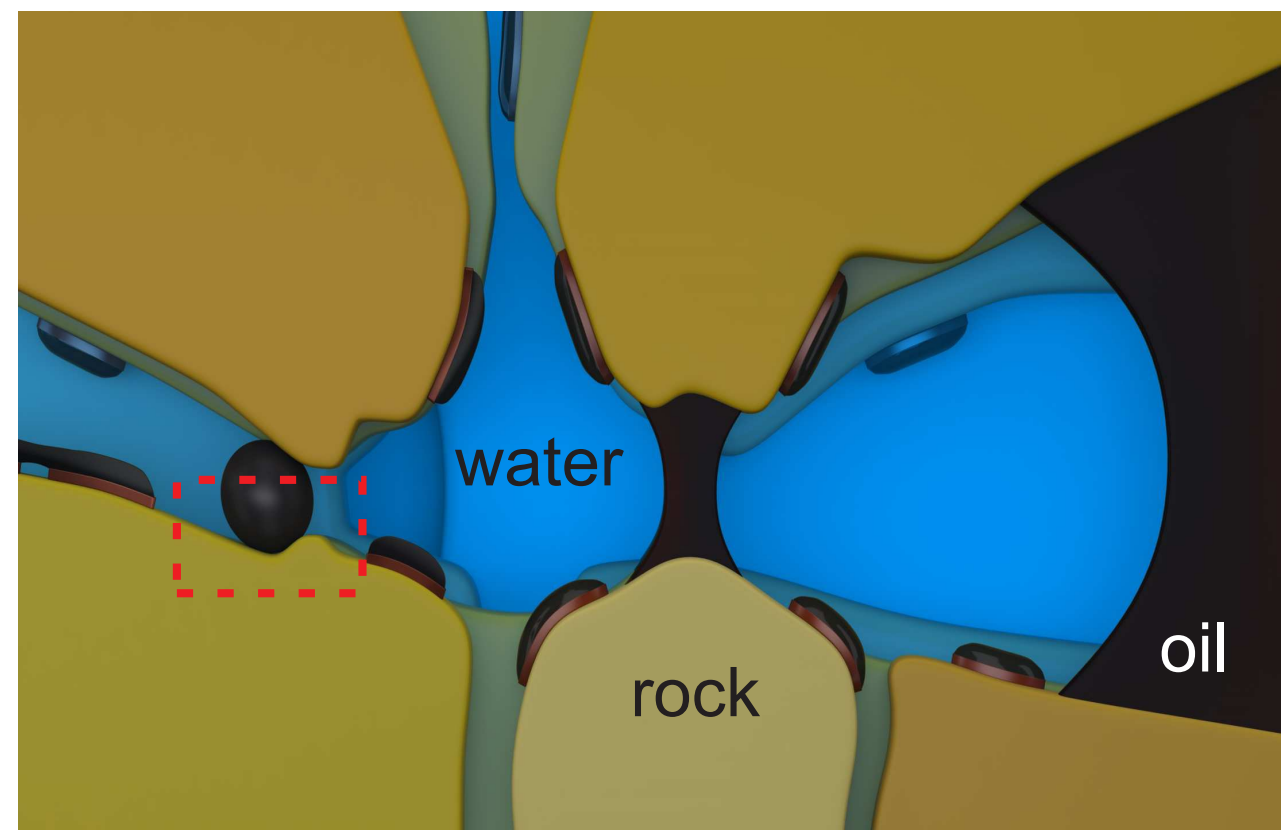

Figure 1.3: Schematic impression of waterflooding with the area of interest for this thesis highlighted.

\subsection{Thesis Scope}

The role of multivalent cations in reservoir wettability and adsorption of polar molecules is much discussed in literature [11, 15, 25]. But most of these studies are performed on coreflooding set-ups, where the insight about the physicochemical processes exists only through some empirical relations. There has been a few attempts to investigate the influence of ionic composition (in the aqueous phase) upon relevant mechanisms. Investigations have focussed on oil-water interfaces and the influence of ion concentration or polar components in either of these phases [8], but the subsequent effects on wettability have only been speculated. Some of the wetting experiments have been done with electrowetting [32], where the consequences of contact angle change using an electric field have been studied in a morphological representation of the pore-space or in a microchannel [33], but electrowetting has its shortcomings in mimicking the wetting phenomena of an oil-reservoir, since it cannot completely replace geometrical and chemical influences. Hence, a detailed investigation of mineral wettability without 


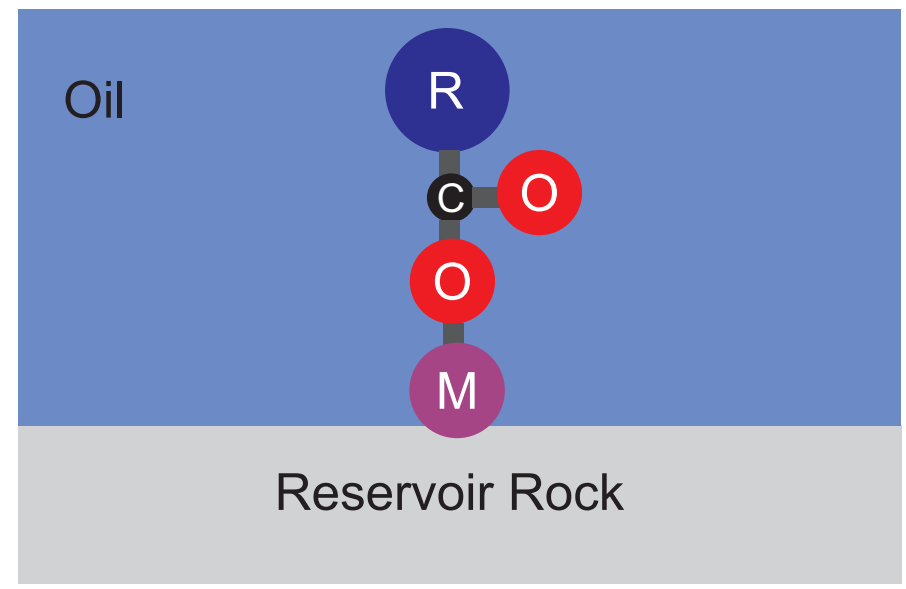

Figure 1.4: Schematic of polar molecule (in this case an organic acid R-COOH) binding to the mineral of the rock surface aided by cation $(\mathrm{M})$ bridging.

the influence of an external electric field is a necessity. The goal of such an investigation will be to systematically verify specific effects by choosing idealized model systems. Although these idealized systems eliminate much of the complexity of a real system, they allow us to demonstrate certain mechanisms very clearly. Such a study does not only stem from an oil recovery motivation, but also reflects upon fundamental processes in a colloidal system, especially at interfaces, e.g., brine solution for oil recovery only contains a few specific types of ion depending on the seawater. However, the entire range of alkali metals and earth alkali metals offer many exciting physicochemical possibilities to observe and understand, as seen in the Hofmeister series. We want to examine the wetting phenomena in a broader sense, to gain a better understanding of the fundamental physical processes. Last but not least, polar molecules add a very different dimension to the adsorption problem. This is depicted in Figure 1.4, where the affinity of an organic headgroup to the mineral surface (or the clay particles attached to it) is illustrated. This affinity is also influenced significantly by the type of ions present in the aqueous phase. In a recent work [34], the effect of specific cations on the stability of organic layers on a mineral surface is investigated. In this thesis, we are more interested in the in-situ binding of these polar molecules to the mineral surface, and the role of cations thereupon. 


\subsection{Thesis Outline}

Wetting is the central theme of this thesis. We investigate wetting properties of a system representative of the oil recovery problem, and subsequently trace the roots of these properties to the fundamental interactions between adsorbing molecules. We focus on sandstones, which usually consist of quartz (silicate) and feldspar (alluminosilicate). Hence, we choose negatively charged mineral surfaces such as silica and mica. The brine of waterflooding is represented by aqueous salt solutions. Instead of only using the ions relevant for simulating oil recovery, we will investigate a broader set of ionic species and systems. In reality, crude oil contains various alkanes along with napthenes and asphaltenes. However, we simplify the system and only consider alkanes in the wetting problem. The polar components of the crude oil have been represented by organic acids ( $\mathrm{R}-\mathrm{COOH})$, with various aliphatic chain lengths. The wetting transition is usually studied with change of temperature, but in recent literature, the role of electrolyte interactions in wetting transition has been identified [35,36]. We investigate wetting transition by just varying the various ionic species in the system. Changing the ionic species results in a change in various short and long range interactions, which we study in a systematic manner, modelling the various aspects of the experimental observations.

In Chapter 2, we review the basic concepts of wetting and adsorption. Following the line of classic textbooks and publications in the area, we introduce the macroscopic concepts related to wetting, such as surface tension, Laplace pressure etc. Thereafter, we explain the thermodynamic origin of wetting starting from adsorption, disjoining pressure. The various contributions in the disjoining pressure are reviewed, namely the van der Waals attraction, hydration repulsion but most importantly, the electric charge contributions. The basics of different types of wetting (complete, partial) are discussed with the emphasis upon the conditions to switch from one to another.

In Chapter 3, we discuss the experimental techniques used in this work. A significant amount of this thesis deals with the measurement of small contact angles using optical methods. Here, we explain in detail the additional steps and care taken, in order to create identical experimental conditions during the measurements, which allows us to measure relative trends in very small contact angles. 
Chapter 4 addresses the competitive wetting of water and oil on a mineral surface in ambient oil (alkane). Here, we address the basic question of wettability alteration on mineral surfaces without the influence of any polar components in the oil. We vary the ionic composition of the aqueous phase and study the subsequent changes in wettability. Subsequently, we focus on the microscopic origins of the observed phenomena. We build a consistent scenario leading from ion adsorption at the solid-liquid interface to charge reversal on the mineral surface and from there to wettability alteration. The wetting films are probed (using ellisometry) for looking into various wetting configurations. The reversal of surface-charge is investigated using streaming potential measurements and atomic force microscopy (AFM). Our results provide a clear mechanistic picture of adsorption and wetting for an oil-reservoir like system, including in particular the relevance of divalent cations, clays and $\mathrm{pH}$.

Chapter 5 continues in the same direction of investigation, but with a theoretical approach. We derive a model that provides a quantitative description of the experimentally observed contact angles. The model is based upon a mean-field electrostatics (Poisson-Boltzmann, PB) approach, but other contributions in the disjoining pressure are included as well. The PB equation is solved numerically with constant charge boundary conditions. Subsequently, we apply a linearized charge regulation model, which is justified for several systems of scientific interest, and allows a unified description of different solid substrates. We obtain analytical expressions for the threshold conditions for a switch from an oil-wet to a water-wet behavior, and the value of contact angles for the finite wettability configurations. This approach describes each interface with only two parameters, which have a clear physical meaning and is shown to be related to the chemistry of each substrate.

In Chapter 6, we proceed further in our investigation of non-electrostatic interactions contributing to wetting alteration. In Chapter 4, the major focus in wetting experiments lies in the valency of the ion-species and the possibility of charge-reversal. For the theoretical model in Chapter 5, standard expressions for van der Waals and hydration forces are used in order to calculate the disjoining pressure. The specific interaction of ions with macromolecules and solid-liquid interfaces is of crucial importance not only in oil recovery but also in biochemistry, colloid science, and engineering. Here, we 
vary the types of cations in the salt (and two anions), their concentrations as well as $\mathrm{pH}$ to investigate a change in wetting properties even though the cation-valency remains same, i.e., a change governed by specific ion effects. Subsequently, we discuss and rationalize the experimental results from a specific adsorption point of view. The majority of investigations into the Hofmeister series talks about monovalent alkali metal adsorption and it is acknowledged that the binding of divalent cations deserves more attention. We attempt to do that by looking at the wettability alteration in our system for divalent earth alkali cations.

Until Chapter 6, we investigate wetting of a mineral substrate due to adsorption from an aqueous phase in pure alkanes as the ambient phase, without the presence of any organic polar molecules. However, while setting the scene in this chapter, we have pointed out the crucial role that the polar components of oil play through their affinity to clay particles, or spontaneous adsorption to the mineral surface or cation bridging etc. In Chapter 7 , we introduce polar molecules to the system in the form of surfactants. The surfactants chosen for the experiments are organic fatty acids, which are a simplified representation of the polar molecules in crude oil. We investigate how the wettability alteration process depends on the adsorption of these amphiphilic molecules. Often a wettability alteration in the presence of such polar components involves the spontaneous retraction of the contact line, leading to an increase of contact angle which is known as autophobing. We investigate autophobing and the influences thereupon of the experimental parameters such as the concentration of the amphiphilic molecules and the salt, nature of alkane and the solid. In order to support the interpretations of our experimental observations, we also develop a model involving the kinetics and final equilibirum stage of the autophobing drop. We attempt to capture the time-scale and final contact angle of the autophobing experiments using this model.

In Chapter 8, we describe the contribution of this thesis in brief and lay out the structure of possible extensions based on this work. The effect of wettability change observed in this work can be implemented suitably in lab-scale oil recovery experiments as well as microfluidic devices. The premises of such possible investigations are explored. 


\section{Bibliography}

[1] L. W. Lake. Enhanced oil recovery. Prentice Hall, 1989.

[2] E. C. Donaldson, G. V. Chilingarian, and T. F. Yen. Enhanced Oil Recovery, I: Fundamentals and Analyses. Elsevier Science, 1985.

[3] T. J. Mays. A new classification of pore sizes, volume 160, pages 57-62. Elsevier, 2007.

[4] D. W. Green and G. P. Willhite. Enhanced Oil Recovery, volume 6. SPE Textbook Series, USA, 1998.

[5] M. J. Rosen, H. Wang, P. Shen, and Y. Zhu. Ultralow interfacial tension for enhanced oil recovery at very low surfactant concentrations. Langmuir, 21(9):3749-3756, 2005.

[6] S. G. Sayegh and D. B. Fisher. Enhanced oil recovery by $\mathrm{CO}_{2}$ flooding in homogeneous and heterogeneous 2D micromodels. Journal of Canadian Petroleum Technology, 48(8), 2009.

[7] A. Muggeridge, A. Cockin, K. Webb, H. Frampton, I. Collins, T. Moulds, and P. Salino. Recovery rates, enhanced oil recovery and technological limits. Philosophical Transactions of the Royal Society aMathematical Physical and Engineering Sciences, 372(2006), 2014.

[8] R. de Ruiter, R. W. Tjerkstra, M. H. G. Duits, and F. Mugele. Influence of cationic composition and ph on the formation of metal stearates at oil-water interfaces. Langmuir, 27(14):8738-8747, 2011.

[9] H. Aksulu, D. Hamso, S. Strand, T. Puntervold, and T. Austad. Evaluation of low-salinity enhanced oil recovery effects in sandstone: Effects of the temperature and ph gradient. Energy \& Fuels, 26(6):3497-3503, 2012.

[10] J.J. Sheng. Critical review of low-salinity waterflooding. Journal of Petroleum Science and Engineering, 120:216 - 224, 2014. 
[11] G. Tang and N. R. Morrow. Influence of brine composition and fines migration on crude oil/brine/rock interactions and oil recovery. Journal of Petroleum Science and Engineering, 24(2-4):99-111, 1999.

[12] D. J. Ligthelm, J. Gronsveld, J. Hofman, N. Brussee, F. Marcelis, and H. van der Linde. Novel waterflooding strategy by manipulation of injection brine composition. Society of Petroleum Engineers, Amsterdam, The Netherlands, June 2009.

[13] A. Lager, K. J. Webb, C. J. J. Black, M. Singleton, and K. S. Sorbie. Low salinity oil recovery - an experimental investigation1. Petrophysics, 49:28-35, 2006.

[14] P. L. McGuire, J. R. Chatham, F. K. Paskvan, D. M. Sommer, and F. H. Carini. Low salinity oil recovery: An exciting new EOR opportunity for alaska's north slope. Society of Petroleum Engineers, Irvine, California, March 2005.

[15] J. S. Buckley and N. R. Morrow. Characterization of crude oil wetting behavior by adhesion tests. Society of Petroleum Engineers, April 1990.

[16] General Electronics. Water injection technology and process solutions, 2014.

[17] X. Zhao, M. J. Blunt, and J. Yao. Pore-scale modeling: Effects of wettability on waterflood oil recovery. Journal of Petroleum Science and Engineering, 71(3-4):169-178, 2010.

[18] M. J. Blunt and H. Scher. Pore-level modeling of wetting. Physical Review E, 52(6):6387-6403, 1995. PRE.

[19] B. Bera, N. S. K. Gunda, S. K. Mitra, and D. Vick. Characterization of nanometer-scale porosity in reservoir carbonate rock by focused ion beam-scanning electron microscopy. Microscopy and Microanalysis, 18(1):171-178, 2012.

[20] B. Bera, S. K. Mitra, and D. Vick. Understanding the micro structure of berea sandstone by the simultaneous use of micro-computed tomography (micro-CT) and focused ion beam-scanning electron microscopy (FIB-SEM). Micron, 42(5):412-418, 2011. 
[21] A. C. Gunde, B. Bera, and S. K. Mitra. Investigation of water and $\mathrm{CO}_{2}$ (carbon dioxide) flooding using micro-CT (micro-computed tomography) images of berea sandstone core using finite element simulations. Energy, 35(12):5209-5216, 2010.

[22] N. S. K. Gunda, B. Bera, N. K. Karadimitriou, S. K. Mitra, and S. M. Hassanizadeh. Reservoir-on-a-chip (ROC): A new paradigm in reservoir engineering. Lab on a Chip - Miniaturisation for Chemistry and Biology, 11(22):3785-3792, 2011.

[23] M. J. Blunt. Flow in porous media - pore-network models and multiphase flow. Current Opinion in Colloid \& Interface Science, 6(3):197-207, 2001.

[24] J. S. Buckley, Y. Liu, and S. Monsterleet. Mechanisms of wetting alteration by crude oils. SPE Journal, 3, 1998.

[25] N. R. Morrow, G. Q. Tang, M. Valat, and X. Xie. Prospects of improved oil recovery related to wettability and brine composition. Journal of Petroleum Science and Engineering, 20(3-4):267-276, 1998. Export Date: 4 November 2015.

[26] P.G. de Gennes. Wetting: statics and dynamics. Rev. Mod. Phys., 57(3):827-863, 1985.

[27] D. Bonn, J. Eggers, J. Indekeu, J. Meunier, and E. Rolley. Wetting and spreading. Reviews of Modern Physics, 81(2):739-805, 2009.

[28] I. Siretanu, D. Ebeling, M. P. Andersson, S. L. S. Stipp, A. Philipse, M.A. Cohen Stuart, H.T.M. van den Ende, and F. Mugele. Direct observation of ionic structure at solid-liquid interfaces: a deep look into the stern layer. Scientific Reports, 4:4956, 2014.

[29] T. Fukuma, M. J. Higgins, and S. P. Jarvis. Direct imaging of lipidion network formation under physiological conditions by frequency modulation atomic force microscopy. Physical Review Letters, 98:106101, 2007.

[30] T. Fukuma, T. Ichii, K. Kobayashi, H. Yamada, and K. Matsushige. True-molecular resolution imaging by frequency modulation atomic force microscopy in various environments. Applied Physics Letters, 86(3), 2005. 
[31] M. Ricci, P. Spijker, and K. Voïtchovsky. Water-induced correlation between single ions imaged at the solid-liquid interface. Nature Communications, 5, 2014.

[32] R. de Ruiter. Manipulation of drops with electrowetting: From morphological transitions to microfluidics. Thesis, 2014.

[33] R. de Ruiter, A. M. Pit, V. M. de Oliveira, M. H. G. Duits, D. van den Ende, and F. Mugele. Electrostatic potential wells for on-demand drop manipulation in microchannels. Lab on a Chip, 14(5):883-891, 2014.

[34] N. Kumar, L. Wang, I. Siretanu, M. Duits, and F. Mugele. Salt dependent stability of stearic acid langmuir-blodgett films exposed to aqueous electrolytes. Langmuir, 29(17):5150-5159, 2013.

[35] I. Ibagon, M. Bier, and S. Dietrich. Wetting in electrolyte solutions. The Journal of Chemical Physics, 138(21):214703, 2013.

[36] I. Ibagon, M. Bier, and S. Dietrich. Order of wetting transitions in electrolyte solutions. The Journal of Chemical Physics, 140(17):174713, 2014.

[37] Y. Li. Oil recovery by low salinity water injection into a reservoir: A new study of tertiary oil recovery mechanism. Transport in Porous Media, 90(2):333-362, 2011. 


\section{CHAPTER 2}

\section{Theoretical Background}

\section{Abstract}

In this chapter, we provide a concise overview of the basic concepts of adsorption and wetting. We introduce the parameters related to macroscopic wetting such as surface tension and Young's angle, and explain the molecular origin of these parameters. The molecular approach is further elaborated when we describe wetting from an adsorption perspective, with a detailed discussion about the well-known theory of electric double layers, short range interaction between adsorbing molecules etc. 


\subsection{Introduction}

We introduced wetting in Chapter 1 from a very specific point of view, i.e., the relative wettability of mineral surfaces in an oil-bearing reservoir to water and oil. But the concept of wettability is very general, because wetting (or non-wetting) is omnipresent in our world. While driving a car to work, the windshield needs to be free of any previously fallen raindrop. So the pane is designed in a way to repel the scattered water droplets. However, the same sheet of glass is wetted by the soap solution during cleaning. What is special about adding soap to water which makes it stick to the same piece of glass? At the same time, such wetting examples are from a macroscopic perspective. If we think of the same problem from a microscopic point of view, new questions arise. The drop being repelled from the glass surface implies that the molecules in the aqueous phase do not like to be at the interface with the glass. On the other hand, from the theory of adhesion, or from van der Waals types of interaction, some of the molecules must get adsorbed at the interface. In this chapter, we focus upon the basics of wetting in a systematic manner, first from macroscopic interpretations and then from an adsorption based outlook.

\subsection{Theory of Capillarity}

A gaseous phase consists of molecules at a temperature where the thermal energy of the molecules is greater than the intermolecular attractions. As a result, the molecules are in a 'free' state, running to/away from each other and the gaseous phase does not conform to any specific shape or volume. If we lower the temperature or introduce more molecules (higher density) at the same temperature, then the cohesive energy between the molecules is stronger compared to the kinetic energy of the molecules, and the same phase becomes dense, or condensed. This phase is still disordered and we call it liquid. Both liquids and gases can flow because of the disordered nature of their molecules, and they are called fluids. The competition between thermal disorder and intermolecular attraction play an important role in the bulk of a solid/liquid/gas, but the role of the latter is far more significant when one phase meets another, i.e., at the boundary of two phases called the interface. Capillarity deals with the study of such an interface, between two liquids or a liquid and a gas phase. The concept of surface tension forms one of the cornerstones of capillarity. 


\subsubsection{Surface Tension}

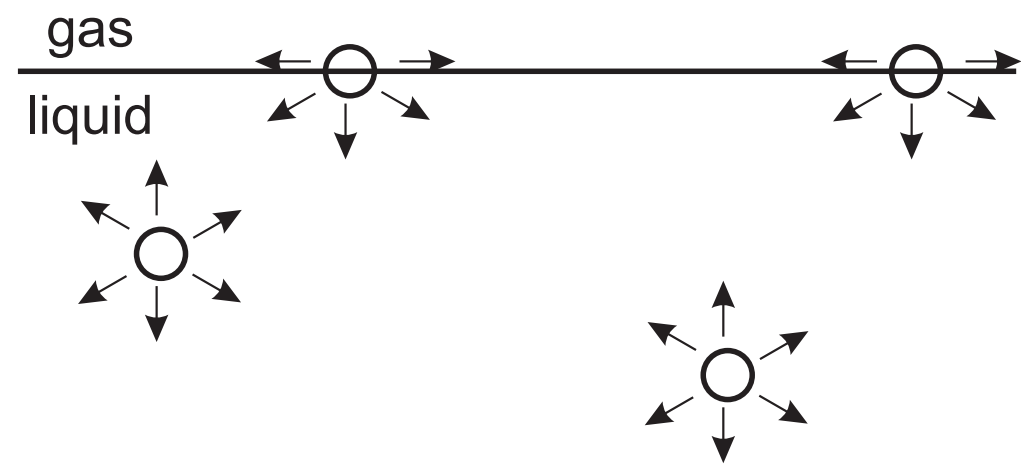

Figure 2.1: Molecular origin of surface/interface tension: molecules in the bulk have uniform interaction around them, while molecules at the boundary miss half of the cohesive interaction from neighboring molecules.

Our hair, when dry, is a thick and full volume. However, after taking a shower, or getting wet in heavy rain, the same mass of hair becomes a drooping flat volume. In almost every aspect of daily life such as this example of dry or wet hair, surface tension plays an important role. The molecules in a bulk condensed phase have uniform cohesive interactions around them, since these forces are distributed equally in the spatial directions from a certain molecule. A molecule in the interface, on the other hand, misses half of this interaction (Figure 2.1), since it has only half of the neighboring molecules compared to in bulk. This situation is portrayed in Figure 2.1, where the phase under consideration has a few molecules at the interface lacking half of their neighbors. This is the reason why a liquid phase always tries to minimize its surface area, so that there is a minimum number of molecules present in the phase with the 'discomfort' of having half of their neighboring molecules. Hence, it requires external work in order to increase the surface area of a liquid phase, and this work/energy per unit area is called the surface tension, usually denoted by $\gamma$. Hence, if the cohesive energy for a molecule in the bulk is represented by $\mathrm{U}$, then the cohesive energy for an identical molecule at the surface will be given by $\frac{U}{2}$. If we assume $a$ as the size of the molecule, and $a^{2}$ as the exposed area of the molecule at the surface, then the excess energy (per unit area) required to bring this molecule from the bulk to the surface (i.e. create the extra bit of surface) is given by: 


$$
\gamma=\frac{U}{2 a^{2}}
$$

Hence, if we ignore entropy, then the surface tension is directly proportional to the cohesive attraction between the molecules of a phase. This is the reason why oils have a lower surface tension, since the cohesive interaction between molecules in oil are of van der Waals type, i.e., $\mathrm{U} \sim \mathrm{k}_{B} \mathrm{~T}$ [1]. Water molecules, on the other hand, have hydrogen bonds between them, increasing the cohesive energy significantly where, $\mathrm{U} \sim 39 \mathrm{k}_{B} \mathrm{~T}$. Hence, the surface tension of water is considerably higher $(\sim 72 \mathrm{mN} / \mathrm{m}$ compared to typical oils with $\sim 20 \mathrm{mN} / \mathrm{m}$ ). To be more precise, molecules at an interface have more freedom since the interface has more entropy. Therefore $\gamma$ depends on $T$, and usually decreases as $T$ increases. A detailed discussion of the relation between surface tension and van der Waals interaction /hydrogen bonding can be found in Lyklema's book [2]. We mention oil and water in this context, since these two phases will be of main importance throughout this thesis.

Surface tension is usually the free energy (reversible work) required to form a surface in air. Interfacial tension (IFT) is the equivalent parameter, when a phase is brought next to a second phase other than air, e.g., the energy required to increase the 'interface' between oil and water is called oil-water interfacial tension $\left(\gamma_{o w}\right)$. We will mainly discuss IFTs throughout this work.

\subsubsection{Laplace Pressure}

The fact that we need to perform some amount of work in order to create a surface/interface implies that there must exist a pressure as we cross a curved interface from one phase to the side of the other phase. This pressure is called the Laplace pressure. We can form an impression about the nature of this pressure by considering the simple case of a spherical oil drop in water [1] (Figure 2.2). The oil drop has an area of $A=4 \pi R^{2}$, where $R$ is the radius of the drop, and the drop volume is $V_{o}=\frac{4}{3} \pi R^{3}$. Any change in the oil drop volume is then replenished by an equal an opposite change in the water volume, i.e., $d V_{o}=4 \pi R^{2} d R=-d V_{w}$. If the o/w interface is stretched outward by an amount $d R$ in an attempt to increase the interface area, then the total amount of work done is: 


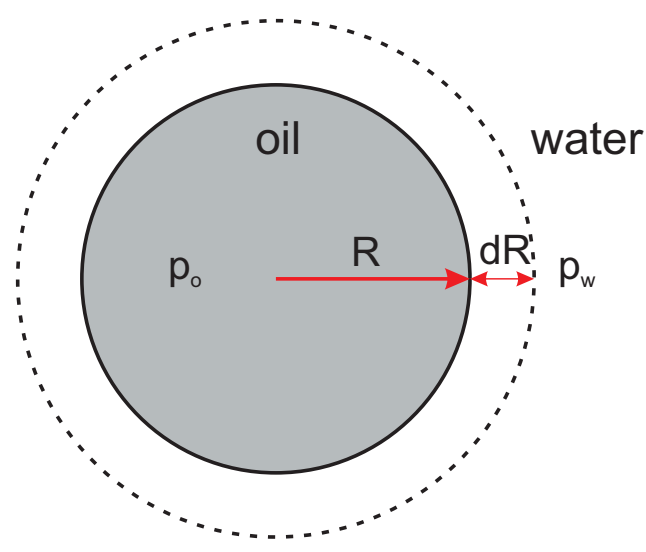

Figure 2.2: Laplace pressure or the excess pressure explained for a spherical oil drop in water. Figure adapted from [1].

$$
\begin{aligned}
\delta W & =\gamma_{o w} d A-p_{o} d V_{o}-p_{w} d V_{w}=0 \\
\text { hence, } \Delta \mathrm{p} & =\left(p_{o}-p_{w}\right)=\frac{2 \gamma_{o w}}{R}
\end{aligned}
$$

As we see, the smaller the drop is, the higher the Laplace pressure becomes and for a typical drop of $1 \mu \mathrm{m}$ radius, the Laplace pressure is of the order of atmospheric pressure. Here we have considered the simplification of a spherical drop. For a similar oil drop in water with a randomly curved interface, the Laplace pressure is given by:

$$
\Delta p=\gamma_{o w}\left(\frac{1}{R_{1}}+\frac{1}{R_{2}}\right)
$$

where $R_{1}$ and $R_{2}$ are the radii of curvature of the interface.

\subsection{Wetting}

We have now introduced the concepts of surface tension, the work required to form an interface and the relevant pressure once this interface is formed. The next question we are interested in follows automatically: what happens when a drop of a liquid (meaning a liquid-air interface or a liquid-liquid interface in case of a liquid ambient phase) is brought in contact with a solid 
phase? The answer can be broadly classified into two categories: either the drop completely spreads on the surface, or does not quite spread on the surface completely. In the latter situation the drop can have many possible shapes (and so can the spreading drop in the former case, we will discuss these in the following sections), but each of these must have a macroscopically visible drop sitting on the solid surface. Such a macroscopic drop in equilibrium has a contact angle, which we will define next.

\subsubsection{Young's Contact Angle}

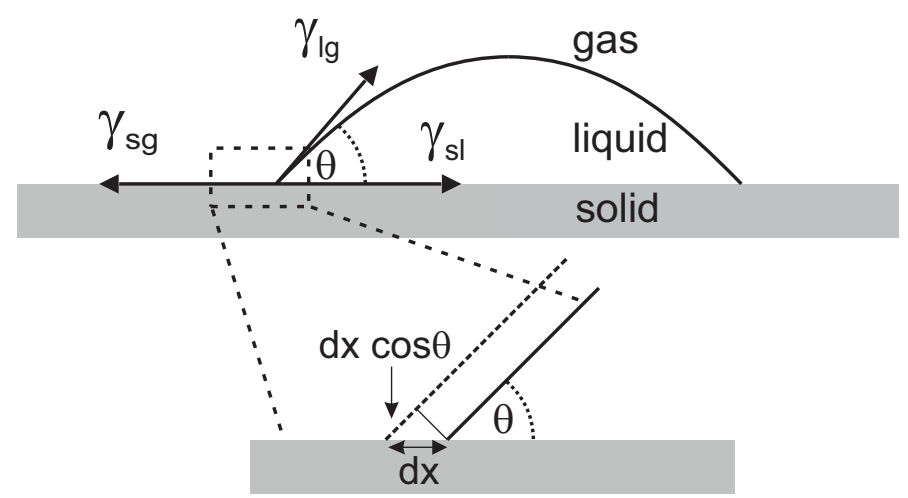

Figure 2.3: A liquid drop on a solid substrate in ambient gaseous phase with a magnified view of the three-phase contact line shown below, where a small change in contact line $d x$ is depicted. The contact angle $\theta$ follows from the energy considerations.

The way this drop behaves on an undeformable solid surface is governed by the surface energies of all the three phases concerned. In Figure 2.3, such a drop on a solid (in ambient air) is drawn with all relevant interfacial tensions shown. If there is a small change in the contact line position by $d x$, then the total energy change $d E$ is given by:

$$
d E=\left(\gamma_{s l}-\gamma_{s g}\right) d x+\gamma_{l g} \cos \theta d x
$$

where, $\gamma_{a b}$ denotes the interfacial tension between phases a and b; In our example, $s, l$ and $g$ are representative of solid, liquid and gas phases, respectively. Since at thermodynamic equilibrium, the energy change $d E=0$, we obtain:

$$
\cos \theta=\frac{\gamma_{s g}-\gamma_{s l}}{\gamma_{l g}}
$$


The angle $\theta$ is named after Thomas Young following his famous work on this topic [3]. The vertical component of the liquid-gas interfacial tension $\left(\gamma_{l g} \sin \theta\right)$ is nullified by the tensile strength of the solid surface on which the drop rests. It is important to realize that at equilibrium, the solid, liquid and the gas phases all co-exist at the three-phase contact line, but this is a special case. For a drop when the spreading is ongoing, the concept of spreading parameter (or the spreading tension) is more appropriate.

\subsubsection{Spreading Parameter}

For a spreading drop, we are interested in the driving force as well as the speed of this spreading process. The rate of the process is directly governed by the driving force (unbalanced sum of the interfacial tension contributions) and any force resisting the spreading. For our system introduced in Figure 2.3, this unbalanced sum of interfacial tension is called the spreading parameter $(\mathrm{S})$, which means, that at a point when the drop has spread considerably (i.e., $\theta \sim 0$ ), the spreading parameter is given by:

$$
\begin{aligned}
S & =\gamma_{s g}-\gamma_{s l}-\gamma_{l g} \\
\text { or }, S & =\gamma_{s g}-\left(\gamma_{s l}+\gamma_{l g}\right)
\end{aligned}
$$

Hence, if $S>0$, then $\gamma_{s g}>\left(\gamma_{s l}+\gamma_{l g}\right)$, and the solid surface, which always prefers the phase(s) with minimizing contribution of surface energy, prefers a layer (film) of the liquid phase on it, rather than forming the solidgas interface which costs more energy. This situation is associated with the complete wetting case and occurs on high-energy surfaces. On the other hand, if $S<0$, then $\gamma_{s g}<\left(\gamma_{s l}+\gamma_{l g}\right)$, then it is favorable for the solid phase to remain in the vicinity of the gas phase. In this case, the liquid phase forms a lens-like drop on the solid surface with a macroscopic contact angle and the situation is associated with partial wetting.

\subsection{Adsorption and Wetting}

Until now, we have discussed the concept of wetting and spreading from a macroscopic point of view i.e., along the line of global energy parameters such as surface tension. Very close to the three phase contact line, the local contact angle is affected by molecular interactions. Although the local contact angle always follows Young's equation, in this case the interfacial 
tensions themselves depend on the contributions of various molecular interactions. We are interested in investigating these molecular interactions which are relevant at colloidal scale (van der Waals or double layer forces, e.g.) and their influence on wetting. In order to discuss the basic concepts required for this investigation, we will revisit wetting in the following section from an adsorption point of view.

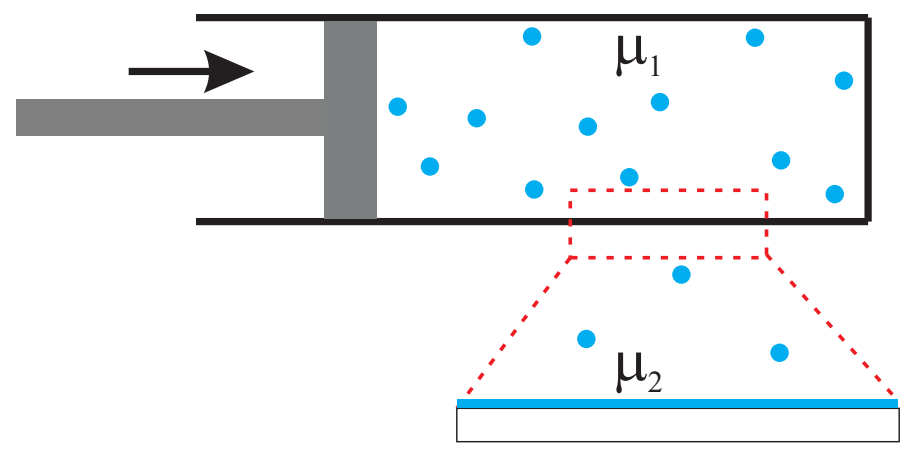

Figure 2.4: A schematic of adsorption induced wetting: a container with solid wall and a piston (of same material as the solid wall) contains gas molecules at chemical potential $\mu_{1}$. If $\mu_{1}$ is increased using the piston, then gas molecules adsorb (enlarged schematic) at solid interface and a liquid film $\left(\mu_{2}\right)$ might form.

Figure 2.4 shows a container, where the wall of the container is made of the solid phase of interest. One of the walls can be used as a piston, so that the pressure in the container can be altered. We introduce a known amount of gas (we call it phase ' 1 ') at a specific temperature $T$ in this container, which gives rise to a chemical potential $\mu_{1}$ (or alternatively, a pressure $P$ ). Here, chemical potential $\mu_{i}$ is defined as the ratio of Gibbs' free energy change in the system and change in number of molecules: $\mu_{i}=\frac{\delta G}{\delta N_{i}}$. There is attractive interaction between the molecules of the gas and the solid of the wall, and hence adsorption can take place at the solid/gas molecules forming the interface. Due to the adsorption, a dense phase (liquid or solid) will appear on the wall of the container. For the rest of our discussion, we will only consider a temperature where a liquid (phase ' 2 ') film appears on the solid wall due to the adsorption process. If we increase the chemical potential $\mu$ in the container (by controlling the piston i.e., by changing the pressure), then co-existence between the bulk gas and the liquid is approached $\left(\mu_{1}=\mu_{2}\right.$, henceforth referred to as $\mu_{s a t}$ ). Hence, two possibilities arise: firstly, layer thickness grows indefinitely as $\mu_{2}$ is increased up to $\mu_{2}=\mu_{1}$ and becomes 
macroscopically thick. This situation is shown in Figure 2.5 by the curve CW. The second possibility is that as $\mu_{2}$ is increased, adsorption $\Gamma$ can reach a finite limiting value $\left(\Gamma_{0}\right)$. Subsequently, if the chemical potential is increased infinitesimally and just exceeds the $\mu_{\text {sat }}$, then macroscopic drops are formed from the additional condensed vapor. This situation is portrayed by the curve PW in Figure 2.5, and in this case the film of adsorbed molecules (in liquid form) has a microscopic thickness which corresponds to the adsorbed amount $\Gamma_{0}$.

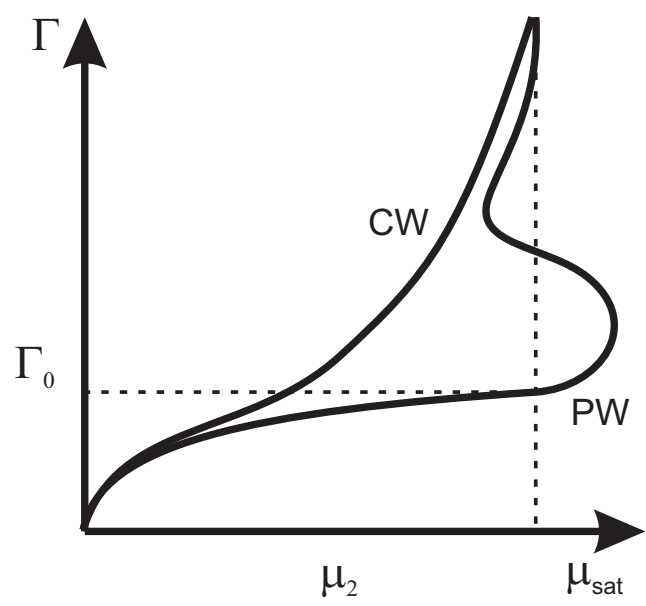

Figure 2.5: Adsorption isotherms leading to complete wetting (CW) and partial wetting (PW). Figure adapted from [2].

Hence, the issue of wetting is related to the question of thermodynamic stability of the adsorbed liquid phase when liquid-gas co-existence is approached. In the case that the wall/gas interface acts as a nucleation site for the liquid, the liquid phase will form due to the adsorption process. At a higher pressure (of liquid/gas co-existence), the adsorbed liquid film thickens, and upon inspection, we can observe this film and say that the wall is wetted. On the other hand, if the solid/gas interface does not act as the nucleation site of the liquid phase, then the liquid film is very thin and is not possible to identify except with the help of specific instruments. In this case, the wall is not wetted. Naturally, the question follows: how are these wetted and non-wetted states associated with the energy/disjoining pressure profiles in the system? We are going to discuss the disjoining pressure in detail, but it is worthwhile to spend a section about the basics of adsorption and how it relates to the free energy, since we are going to use these in our 
modeling, especially in the surface complexation model and in surfactant induced wetting.

\subsection{Basics of Adsorption}

We have discussed already that a molecule close to an interface behaves differently compared to a molecule in the bulk. If we consider a molecule in our gas phase or in the formed liquid phase, then this molecule has a different binding energy at the solid-liquid interface compared to the bulk. Furthermore, the spatial freedom of the molecule is also less at the interface compared to the bulk, since the adsorbed molecule can only move around in the two dimensional surface and not in the three dimensional bulk. This results in a reduction of entropy for the adsorbed molecule. Based on changes in entropic and enthalpic contributions of the free energy, the molecule is adsorbed or desorbed at the interface. A decrease in the free energy leads to adsorption, while an increase in the free energy leads to desorption of the molecule from the interface.

\subsubsection{Langmuir Adsorption}

Figure 2.6a represents the picture of such molecules getting adsorbed at the wall of our container, or being desorbed from the wall. Here, we consider the case of liquid at the wall (having been formed already at the solid/gas interface) and furthermore, we consider liquids containing solutes, since this will be the case in all our experimental investigations throughout the thesis. In order to relate the adsorption (of the solute) to the bulk concentration $c$ of the solute, we can represent the adsorption/desorption reaction as:

$$
S X \rightleftharpoons S+X
$$

where $S$ represents the surface site $S$ able to form the complex SX with the adsorbing species $X$. The reversible sign indicates a dynamic equilibrium between adsorbing and desorbing species. For various solid surfaces of our interest, the total available sites $\left(\Gamma_{0}\right)$ on the solid surface is known from crystallography, while the available sites at a liquid/liquid interface is calculated from maximum packing density at this interface [4]. We consider that at a specific instant $t$, a $\Gamma$ sites are filled with adsorbing $X$ molecules. Then we 


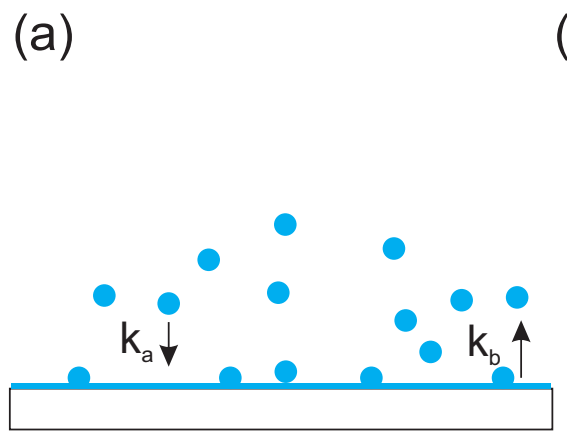

(b) $\Gamma / \Gamma_{0}$

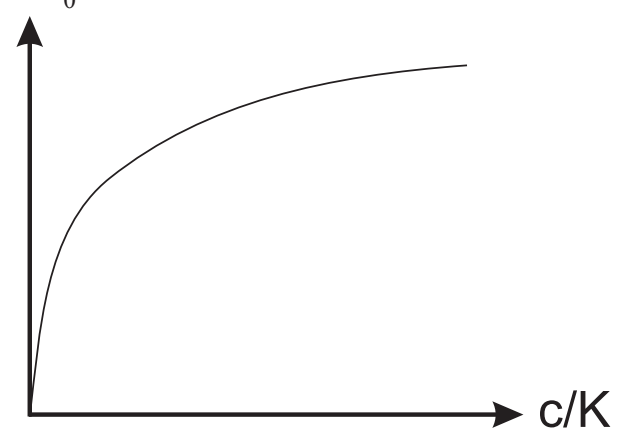

Figure 2.6: (a) A schematic of adsorption and desorption at solid/gas interface in our container from Figure 2.4 with the rate constants of adsorption and desorption shown (b) a typical Langmuir-type adsorption isotherm.

can write the rate of adsorption as:

$$
\begin{aligned}
j_{a d s} & =k_{a} c\left(\Gamma_{0}-\Gamma\right) \\
o r, j_{a d s} & =k_{a} c\left(1-\frac{\Gamma}{\Gamma_{0}}\right) \Gamma_{0} \\
o r, j_{a d s} & =k_{a} c(1-\alpha) \Gamma_{0}
\end{aligned}
$$

where, $k_{a}$ is the adsorption rate constant. On the other hand, desorption from the interface does not depend on the bulk concentration of the solute, but just on the number of sites which are already filled by adsorption. Hence, the rate of desorption can be defined as:

$$
j_{\text {des }}=k_{d}\{S X\}=k_{d} \Gamma
$$

where, $k_{d}$ is the desorption rate constant. When equilibrium is achieved, the adsorption and desorption rate are equal. This leads to:

$$
\begin{aligned}
j_{a d s} & =j_{d e s} \\
\text { or, } k_{a} c(1-\alpha) \Gamma_{0} & =k_{d} \Gamma \\
\text { or, } k_{a} c(1-\alpha) & =k_{d} \frac{\Gamma}{\Gamma_{0}}=k_{d} \alpha \\
\text { or }, \alpha & =\frac{\Gamma}{\Gamma_{0}}=\frac{\frac{c}{K}}{1+\frac{c}{K}}
\end{aligned}
$$

where, $K=\frac{k_{d}}{k_{a}}=\frac{\{S\}[X]}{\{S X\}}$ is the equilibrium adsorption/desorption constant. The relation derived in Equation 2.10 is the standard expression for a Langmuir type adsorption isotherm [5, 6,7] depicted in Figure 2.6b. In this type of 
adsorption/desorption, the molecules do not interact with each other. Such interaction between the molecules is considered in Frumkin type isotherm [5], though we will not discuss that in detail since our models in this thesis are based on Langmuir type adsorption.

It is also important to understand the relevance of $K$ from a surface reaction point of view, as we have written in Equation 2.7. From law of mass action:

$$
K\{S X\}=\{S\}[X]
$$

where, $\{A\}$ and $[\mathrm{A}]$ denote the surface and the bulk concentrations of a species, respectively. The volume concentration of species $X$ is the same as the bulk concentration $c: c=[X]$. Since, the total site density $\Gamma_{0}$ of the crystalline solid is known, the surface concentrations of the species $S$ and $S X$ can be expressed as: $\{S\}=\Gamma_{0}-\Gamma$ and $\{S X\}=\Gamma$. Hence, we can write: $\Gamma_{0}=\{S X\}+\{S\}$. We can work out the equilibrium state in the same manner:

$$
\begin{aligned}
j_{a d s} & =k_{a}[X]\{S\} \\
j_{\text {des }} & =k_{d}\{S X\} \\
j_{a d s} & =j_{\text {des }} \\
\text { Hence, } \mathrm{K} & =\frac{k_{d}}{k_{a}}=\frac{\{S\}[X]}{\{S X\}}
\end{aligned}
$$

In Chapter 5, we use the approach of this mass-action law to express the surface charge of a species with just the input of $p K=-\log _{10} K$.

\subsubsection{Adsorption and Surface Tension}

Since we have described wetting from a macroscopic surface tension perspective and subsequently discussed the details of adsorption, it is evident that the two are connected to each other. At equilibrium (i.e., when the bulk solute concentration $c$ defines the chemical potential $\mu$ ), the change in surface tension is given by the Gibbs adsorption equation: $d \gamma=-\Gamma d \mu$. But the bulk and the surface are equilibrated as parts of the total system which we consider to be a grand canonical ensemble. Hence, we write:

$$
\begin{aligned}
\mu & =\mu_{0}+k_{B} T \ln (c) \\
\mathrm{d} \mu & =k_{B} T \mathrm{~d} \ln (c)
\end{aligned}
$$


We can replace Equation 2.12 in the relation $d \gamma$ :

$$
\begin{aligned}
\mathrm{d} \gamma & =-k_{B} T \Gamma \mathrm{d} \ln (c)=-\frac{k_{B} T \Gamma}{c} \mathrm{~d} c \\
\text { but, } \frac{\Gamma}{\Gamma_{0}} & =\frac{\frac{c}{K}}{1+\frac{c}{K}} \\
\text { hence, } \gamma(\mathrm{c}) & =\gamma_{0}-k_{B} T \Gamma_{0} \ln \left(1+\frac{c}{K}\right) \\
\text { or, } \gamma(\alpha) & =\gamma_{0}+k_{B} T \Gamma_{0} \ln (1-\alpha)
\end{aligned}
$$

to obtain the two-dimensional equation of state for the adsorbing molecules. The relation is comparable to the three-dimensional equation of state, where pressure is replaced by surface tension and volume fraction is replaced by fractional coverage $\left(\alpha=\frac{\Gamma}{\Gamma_{0}}\right)$. The adsorbed molecules at the interface create a two-dimensional pressure (surface pressure) acting in the opposite direction compared to the surface tension. Hence, adsorption of molecules at an interface reduces the surface tension.

\subsubsection{Rate Determining Process}

It takes the adsorbing molecules some time to arrive at the available site and get adsorbed, and for the system to eventually achieve an equilibrium state. Sometimes, we are interested in that time, i.e., how fast does the system reach equilibrium (if it does so at all). When the bulk concentration of solute is higher than the concentration at the interface, diffusive transport of the molecules takes place. In Figure 2.7, we have drawn a schematic illustrating such transport, where the diffusing molecules arrive at the sub-surface layer around the newly formed interface. The following process of adsorption then depends on many things such as molecular interactions or solvation energy. These parameters controlling adsorption from the sub-surface layer to the interface can be termed collectively as an 'adsorption barrier'. But before getting adsorbed, the diffusive transport has to bring the molecules to the sub-surface layer. This transport can be described by Fick's law in one-dimension:

$$
\frac{\partial c}{\partial t}=D \frac{\partial^{2} c}{\partial x^{2}}
$$

with $c$ the sub-surface solute concentration, $D$ the diffusion coefficient and $x$ the distance from the interface. Assuming a bare interface $(\Gamma=0)$ at $t=0$, the boundary condition for the transport equation can be written as: 


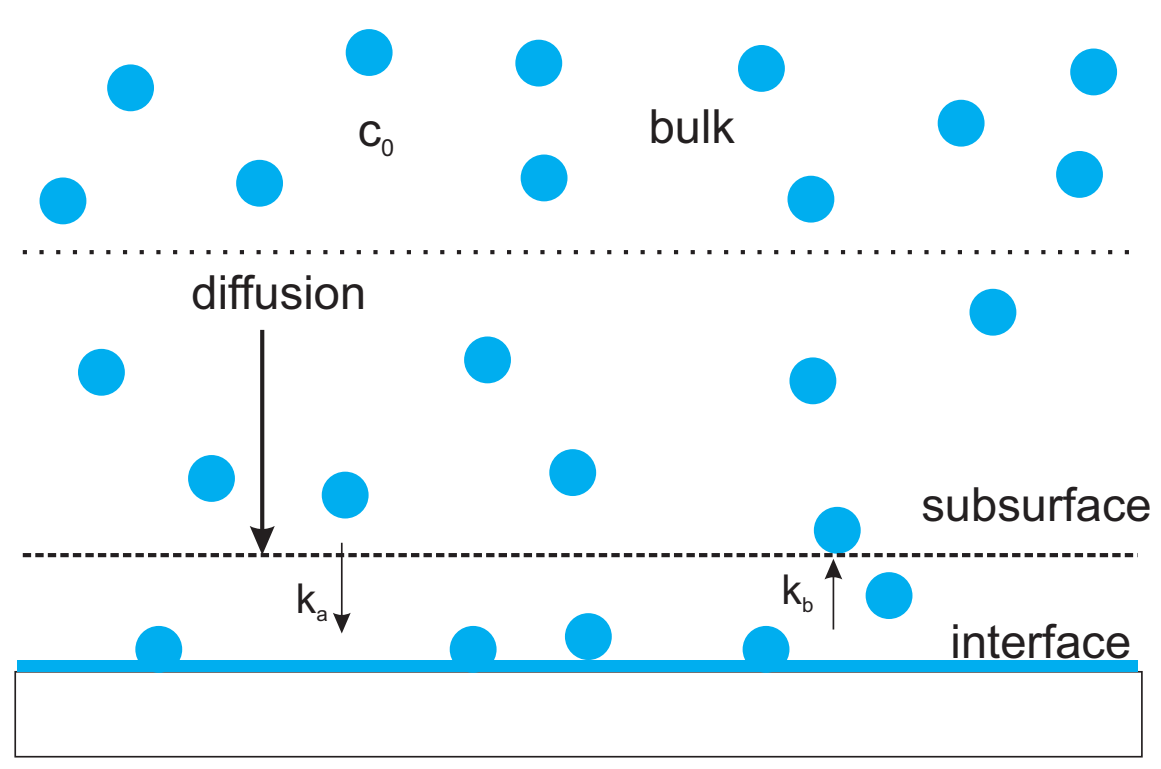

Figure 2.7: Schematic of diffusion of solute from bulk to the subsurface layer and subsequent adsorption to/ desorption from the solid/gas interface. Figure adapted from [8].

$$
\frac{d \Gamma}{d t}=\left.D \frac{\partial c(x, t)}{\partial x}\right|_{x=0}
$$

Hence, solving Equation 2.14 with a Ward-Tordai [9] approach, where we assume $c(0, t)=0$, i.e., the sublayer is completely depleted, we find an expression for coverage at the interface (or the sub-surface):

$$
\Gamma(t)=2 c_{0} \sqrt{\frac{D t}{\pi}}
$$

where, $c_{0}$ is the bulk solute concentration. Hence, the coverage at the subsurface layer increases as $\Gamma \sim t^{1 / 2}$. If this is faster than the subsequent adsorption process guided by the adsorption barrier, then it is adsorption which controls the rate of the whole process. If on the other hand, the bulk concentration is relatively low, then the diffusion process is slower and becomes the rate determining step of the process. 


\subsection{Wetting and Disjoining Pressure}

After we have reviewed the basics of adsorption, we are again ready to pick up our thread of adsorption and wetting. We discussed the formation of an adsorbed liquid layer at solid/gas interface, depending upon the change in chemical potential $\mu$. If the thickness of this liquid film is $h$, then we can visualize the situation like this: the solid/liquid interface already exists, and a liquid/gas interface is brought from infinity to the distance $h$ in order to form this film. This develops an energy of interaction per unit area $\Phi(h)$ (from the total Gibbs energy) and as a result, a pressure across the film, which is known as the disjoining pressure $\Pi(h)$ [10].

$$
\Phi(h)=-\int_{\infty}^{h} \Pi\left(h^{\prime}\right) d h^{\prime}
$$

In the following section, we will discuss about various interactions controlling the nature of this interaction energy (or potential) $\Phi$ and the disjoining pressure $\Pi(h)$. One of the primary interactions in a wetting film originates from the overlapping of the electric double layers, if there are charged species present in the system. Until now, we have described a wetting picture with a pure gas or liquid phase, which will hardly lead to any electric double layer. However, as explained in Chapter 1, we are exclusively interested in the role of ionic species in wetting throughout this thesis. For the following discussion, we assume the liquid phase to contain ions which lead to an electric double layer, at the solid interface, as well as the gas interface.

\subsubsection{Electric Double Layer}

If an adsorbing surface acquires a finite surface charge due to the adsorption/desorption of ions, then we can call it the first layer of charge or the 'Stern' layer. This layer of charge will interact with the charged species in the bulk liquid, meaning they will attract the counter-ions and repel the co-ions. Hence, between the first layer of charge (charged surface) and the bulk liquid, there will be a spatial arrangement of co-ions and counter-ions, which we call the second layer or the 'diffuse layer' of charge. The first and the second layer of charge (or, the Stern and diffuse layers) together create the 'Electric Double Layer' (EDL). Figure 2.8 shows the schematic of a typical double layer at a solid-liquid interface, where the bare solid surface charge, different ionic species in the liquid phase, hydrated ions are shown. It is important to note that, just like a solid/liquid interface, electric double layers 
are also formed at the liquid/liquid interface.

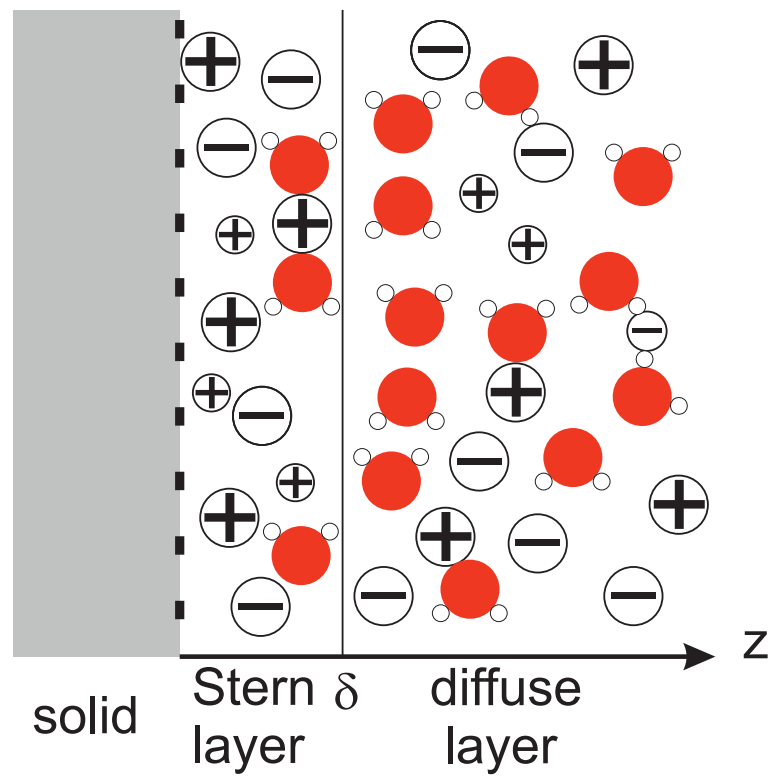

Figure 2.8: Schematic of an electric double layer inspired by the drawings in [11]. Cations and anions of various size are depicted in Stern layer as well as diffuse layer along with water molecules and hydrated ions.

We consider an infinitely large flat plate to represent the problem in 1dimension. For the $i$ th ion species with a valency of $Z_{i}$, the distribution of the ions at thermal equilibrium can be written using Boltzmann distribution as:

$$
c_{i}(z)=c_{i}^{\infty} \exp \left(\frac{-Z_{i} e \phi(z)}{k_{B} T}\right)
$$

where, $c_{i}^{\infty}$ is the bulk ion concentration, $e$ is the electron charge and $\phi(z)$ is the electrostatic potential at distance $z$ from the surface. The behavior of $\phi(z)$ in the neighbourhood of the surface follows the ion-distribution governed by the Poisson law:

$$
\frac{d^{2} \phi(z)}{d z^{2}}=-\frac{\rho_{e l}(z)}{\epsilon \epsilon_{0}}=-\frac{\sum_{i} Z_{i} e c_{i}(z)}{\epsilon \epsilon_{0}}
$$

Combining Equations 2.18 and 2.19, we obtain the non-linear PoissonBoltzmann (PB) equation: 


$$
\epsilon \epsilon_{0} \frac{d^{2} \phi(z)}{d z^{2}}=-e \sum_{i} Z_{i} c_{i}^{\infty} \exp \left(\frac{-Z_{i} e \phi(z)}{k_{B} T}\right)
$$

which assumes slightly different forms for a 1:1 electrolyte (such as $\mathrm{NaCl}$ salt) and a 2:1 electrolyte $\left(\mathrm{CaCl}_{2}\right.$ salt):

$$
\frac{d^{2} \phi(z)}{d z^{2}}=\left\{\begin{array}{lc}
\frac{\kappa_{D}^{2} k_{B} T}{e} \sinh \left(\frac{e \phi(z)}{k_{B} T}\right) & 1: 1 \\
\frac{\kappa_{D}^{2} k_{B} T}{3 e}\left[\exp \left(\frac{e \phi(z)}{k_{B} T}\right)-\exp \left(-\frac{2 e \phi(z)}{k_{B} T}\right)\right] & 2: 1
\end{array}\right.
$$

where, we have introduced the Debye length $\kappa_{D}^{-1}=\left(\frac{\epsilon \epsilon_{0} k_{B} T}{2 I e^{2}}\right)^{1 / 2}, I$ is the ionic strength of the electrolyte: $I=\sum_{i}^{n} c_{i}^{\infty} Z_{i}^{2}$, with $Z_{i}$ the valency of the $i$ th ionic species. This length-scale is indicative of the competition between electrostatic energy and thermal diffusive energy and provides information about the screening length of the double layer. $\kappa_{D}^{-1}$ varies from a few hundred nms in the absence of ionic species in an aqueous phase to a $\mathrm{nm}$ in the presence of salt at $100 \mathrm{mM}$ concentration. The screening length is of considerable importance here, since electrostatic interactions are usually long-range, but here we derive an exponentially decaying short range force which is negligible beyond a few nms. This is because the distribution of ions across the Debye screening length prevents the static electric field from penetrating the solution.

\section{Debye Hückel (DH) Approximation}

The PB equation is a non-linear second order differential equation and requires numerical solution. However, in order to focus on the physical principles, simplifications have been used throughout the history and we will also (in parts) follow these footsteps in Chapter 5. The Debye Hückel approach assumes small electrostatic potentials i.e., $e \phi(z) \ll k_{B} T$. As a result, Equation 2.20 can be simplified to:

$$
\frac{d^{2} \phi(z)}{d z^{2}}=\kappa_{D}^{2} \phi(z)
$$

where a simple superposition of exponential functions can be assumed as a solution and appropriate boundary conditions can be applied in order to find an exact solution. 


\section{Grahame's Equation}

Along with the electrostatic potential in the system, we are (even more frequently) interested in the surface charge in our investigation. There are many challenges in measuring the surface charge directly, but from charge neutrality the diffuse layer charge $\sigma_{d}$ must be equal to the surface charge. We have already defined a charge density for the diffuse layer and the charge can be obtained by integrating this density from the 'inner' edge $(z=\delta)$ to infinity for isolated plates:

$$
\sigma_{\delta}=\int_{\delta}^{\infty} \rho_{e l}(z) d z=\int_{\delta}^{\infty}\left[e \sum_{i} Z_{i} c_{i}^{\infty} \exp \left(\frac{-Z_{i} e \phi(z)}{k_{B} T}\right)\right]
$$

It is possible to couple the PB equation here, and obtain a relation between charge density and potential drop without knowing the exact solution of the PB equation. This relation is known as Grahame's equation [12] and assumes the following forms for $1: 1$ and $2: 1$ electrolytes:

$$
\left(\sigma_{0}+\sigma_{\delta}\right)^{2}= \begin{cases}4 \epsilon \epsilon_{0} k_{B} T c_{i}^{\infty}\left[\cosh \left(\frac{e \phi_{0}}{k_{B} T}-1\right]\right. & 1: 1 \\ 2 \epsilon \epsilon_{0} k_{B} T c_{i}^{\infty}\left[\exp \left(-\frac{2 e \phi_{0}}{k_{B} T}\right)+2 \exp \left(\frac{e \phi_{0}}{k_{B} T}\right)-3\right] & 2: 1\end{cases}
$$

A simpler representation of the electric double layer is the Gouy-Chapman Model, where the Stern layer is ignored. Hence, the interface with its inherent and adsorbed charge is considered the first layer, and the diffuse layer is considered the second layer of the EDL. In this case, the surface charge density is a simpler version of the above equation:

$$
\sigma_{0}^{2}= \begin{cases}4 \epsilon \epsilon_{0} k_{B} T c_{i}^{\infty}\left[\cosh \left(\frac{e \phi_{0}}{k_{B} T}-1\right]\right. & 1: 1 \\ 2 \epsilon \epsilon_{0} k_{B} T c_{i}^{\infty}\left[\exp \left(-\frac{2 e \phi_{0}}{k_{B} T}\right)+2 \exp \left(\frac{e \phi_{0}}{k_{B} T}\right)-3\right] & 2: 1\end{cases}
$$

During calculation of $\sigma_{d}$, we come across the diffuse layer potential $\phi_{d}$ as well, which is usually known as $\zeta$ potential. The $\zeta$ potential can be measured by streaming potential experiments, which we describe in Chapter 3.

\section{DH Equation and Grahame}

Before discussing other contributions, our last remark is about the linearized $\mathrm{PB}$ equation and the simplicity of calculating charge-potential distribution from it. Coupling Equation 2.22 and 2.23, we obtain a linear relationship: 


$$
\sigma_{0}=\epsilon \epsilon_{0} \kappa_{D} \phi_{0}
$$

We will use and discuss this linear relation in detail in Chapter 5.

\subsubsection{Van der Waals Contribution}

Dispersion forces are ubiquitous in a colloidal system. Van der Waals forces are attractive short-range interaction between molecules and the London van der Waals force represents the dispersion part of this interaction in our system of interest. This contribution in a wetting film can be positive or negative depending on the Hamaker's constant A. The corresponding disjoining pressure decays with the third power of film thickness $h$ in the denominator i.e., $\Pi(h) \sim h^{-3}$, and as a result the interaction potential $\Phi(h)$ decays with the square of $h, \Phi(h) \sim h^{-2}$. The other sub-contributions of van der Waals attraction i.e., Keesom part or Debye part are relatively well-defined. But, the dispersion forces are of quantum nature and are quite difficult to interpret, especially, as we will observe in Chapter 6, in the context of our complex solid-water-oil system. As a simplified approach, we have used the $\Phi(h) \sim h^{-2}$ relation in Chapter 5 in order to calculate interaction potential $\Phi(h)$ in the wetting film.

\subsubsection{Solvation and Steric Contribution}

If the wetting film is extremely thin (i.e., $h$ is very small), then the liquid layers close to the boundaries overlap. This leads to extra work being required (by or on the system) for the film to remain stable. This contribution is known as the solvation structure force (or, hydration or hydrophobic force). This force can be approximated with a 'power law' decay, but in recent times, experimental insight $[13,14]$ has pointed to an exponential decay:

$$
\Pi_{\text {solvation }}(h)=\Pi_{\text {solvation }, 0} \exp \left(-\frac{h}{\lambda}\right)
$$

where, $\lambda$ is the parameter representing the decay in the nature of the force. In addition, steric interactions are also present due to adsorption of polymers and polyelectrolytes. In this case, charged interfaces can interact with each other only through a number of (charged) molecules and hence, these molecules alters the nature of the interaction. However, we will not consider them separately in our models in this thesis, and consider that the ex- 
ponential decay of the solvation structure force already considers such steric influences.

\subsection{Nature of $\Phi(h)$ and $\Pi(h)$}

We have introduced the various intrinsic contributions in the disjoining pressure $\Pi(h)$ in the previous section. We can express the total disjoining pressure as:

$$
\begin{aligned}
\Pi_{\text {total }}(h) & =\Pi_{\text {electric }}(h)+\Pi_{\text {vanderWaals }}(h)+\Pi_{\text {solvation }}(h) \\
\text { or, } \Pi(\mathrm{h}) & =\Pi_{\text {el }}(h)+\Pi_{v d W}(h)+\Pi_{\text {solv }}(h)
\end{aligned}
$$

where, $\Pi_{\text {electric }}$ is the pressure originating from the electric double layer.

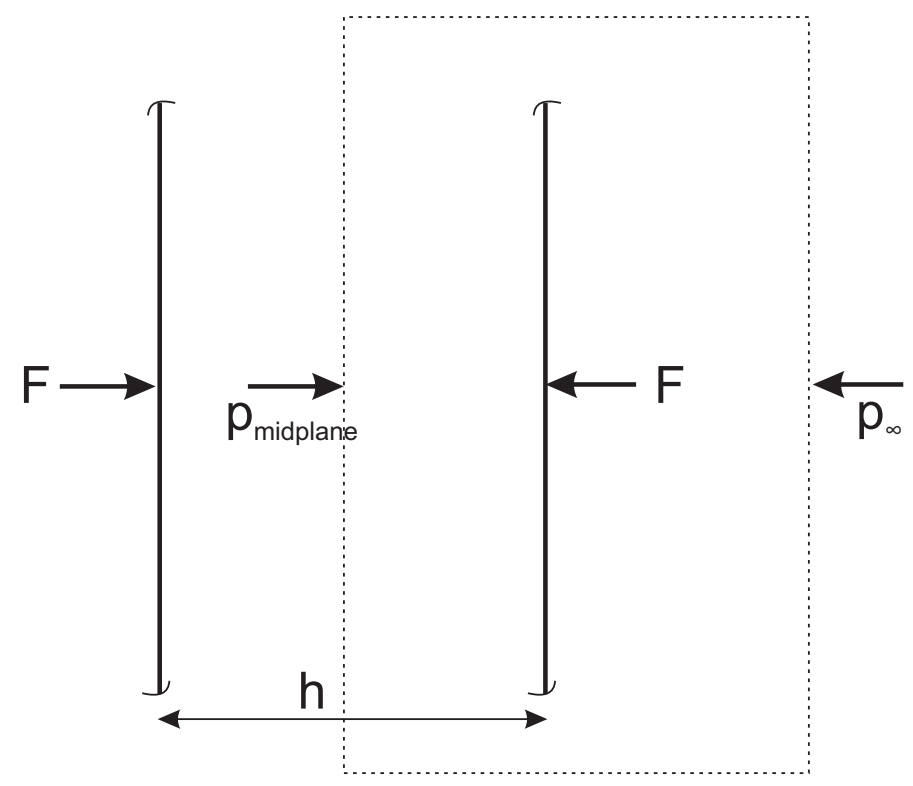

Figure 2.9: Calculation of disjoining pressure $\Pi(h)$ between two charged interfaces.

First, we focus on the nature of $\Pi_{e l}(h)$. We have already discussed the various basics of electrostatic charge and potential in the wetting film between a solid and a gas phase. Figure 2.9 shows a schematic for calculation of the pressure around charged plates [15]. The different electrostatic forces are as follows: (i) force on the adsorbing interface (ii) force on the mid-plane, 
where the electric stress vanishes since the electric field at the midplane is 0 in case of a symmetric system, and (iii) the pressure at infinity. Hence, we can write the total contribution of these forces (per unit area) as follows:

$$
\Pi_{e l, p a r t 1}=\sum k_{B} T c_{i}^{\infty} \exp \left(-\frac{e Z_{i} \phi}{k_{B} T}\right)
$$

in parameters which we have used already. We notice that the whole pressure term in this case is controlled by the concentration of the ionic species and, hence, this is the osmotic pressure term of the disjoining pressure $\Pi(h)$. In other words, $\Pi_{e l, p a r t 1}=\Pi_{e l, o s m o t i c}$. But for two oppositely charged interfaces (which are adsorbing plate-like surfaces), the lack of symmetry around the midplane (i.e., the electric field does not vanish) leads to an extra term known as the Maxwell Stress term:

$$
\Pi_{\text {el,maxwellstress }}=-\frac{1}{2} \epsilon \epsilon_{0} \frac{d^{2} \phi}{d z^{2}}
$$

The total disjoining pressure stems from the fact that this additional stress counters the osmotic repulsion. Hence, combining Equation 2.28 and 2.29 , we retrieve the expression for the disjoining pressure:

$$
\Pi_{e l}=k_{B} T \sum c_{i}^{\infty} \exp \left(-\frac{e Z_{i} \phi}{k_{B} T}\right)-\frac{1}{2} \epsilon \epsilon_{0} \frac{d^{2} \phi}{d z^{2}}
$$

The corresponding electrostatic contribution of the interaction potential $\Phi(h)$ is obtained by integrating this expression across the thickness of the film:

$$
\Phi_{e l}(h)=\int_{h}^{\infty}\left[k_{B} T \sum c_{i}^{\infty} \exp \left(-\frac{e Z_{i} \phi}{k_{B} T}\right)-\frac{1}{2} \epsilon \epsilon_{0} \frac{d^{2} \phi}{d z^{2}}\right] d h^{\prime}
$$

Since we have already introduced the other contributions to the disjoining pressure and interaction potential, we can write:

$$
\Phi(h)=\Phi_{e l}(h)+\Phi_{v d W}(h)+\Phi_{\text {solv }}(h)
$$

which we will use frequently in our models of adsorption induced wetting.

It is possible to measure the disjoining pressure for a wetting film using a surface force apparatus (SFA) or atomic force microscope (AFM). Figure 2.10 shows a very typical $\Phi(h)$ and $\Pi(h)$ curve (adapted from [2]). This type of behavior (of $\Phi(h)$ ) is common for a wetting film on a solid substrate where Derjaguin-Landau-Verwey-Overbeek (DLVO) type of interactions are 


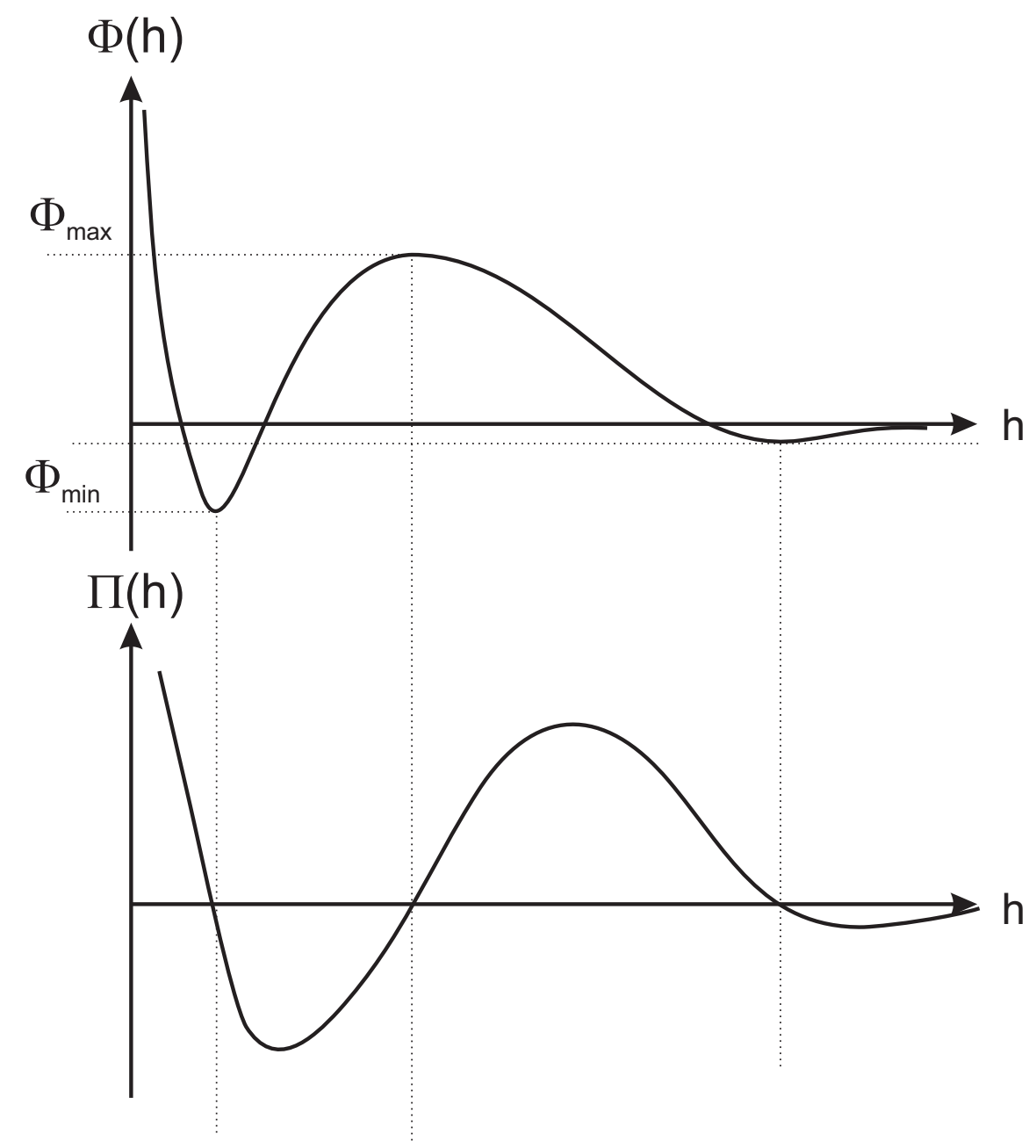

Figure 2.10: A typical DLVO interaction potential $\Phi(h)$ and disjoining pressure $\Pi(h)$ plot between two charged interface. Figure redrawn from [2].

present, i.e., the interactions which we have elaborated above in detail. It is crucial at this point to look at the various wetting configurations which might result, based upon the nature of $\Pi(h)$ or $\Phi(h)$. 


\subsection{Wetting Configurations}

Figure 2.11a shows an asymptotically repulsive interface potential. The minimum energy configuration is attained for $h \rightarrow \infty$, corresponding to a complete wetting scenario. We are going to abbreviate it as CW in the subsequent chapters.

Figure 2.11b shows an interface potential with a minimum at a finite thickness $h_{m i n}$, and an attractive convex profile for large $h$. If the interface potential is convex, it is thermodynamically advantageous for a film of thickness $h_{0}$ to split into two films of thicknesses $h_{1}$ and $h_{2}$ covering fractions $\alpha_{1}, \alpha_{2}$ of the substrate, so that $\alpha_{1}+\alpha_{2}=1$. Due to convexity, we indeed have $\left(\alpha_{1}+\alpha_{2}\right) \Phi\left(h_{0}\right)>\alpha_{1} \Phi\left(h_{1}\right)+\alpha_{2} \Phi\left(h_{2}\right)$. Any film of thickness larger than $h_{\min }$ will thus spontaneously evolve into a macroscopic droplet $(h \rightarrow \infty)$ in equilibrium with a thin film of thickness $h_{\min }$ [1]. Macroscopically it is possible to perceive this drop only, which gives us the impression that this is a partial wetting situation while in reality, this assumption is not completely true. Hence, such a wetting configuration is called 'pseudo-partial wetting' and we will denote it by PP.

It is possible to relate the depth of minimum $\Phi(h)$ with the equilibrium contact angle $\theta_{e q}$. If a macroscopic drop co-exists with a microscopic wetting film of thickness $h$, then for this drop Young's equation holds, i.e., $\gamma_{s g}=\gamma_{s l}+$ $\gamma_{l g} \cos \theta$. However, for the film part, the solid interacts with the gas phase through the liquid and hence, the solid/gas interfacial tension must have a component of the interaction potential $\Phi(h)$ from the film. This implies that $\gamma_{s g, f i l m}=\gamma_{s l}+\gamma_{l g}+\Phi\left(h_{m i n}\right)$. Hence we can write:

$$
\begin{aligned}
\gamma_{s l}+\gamma_{l g} \cos \theta & =\gamma_{s l}+\gamma_{l g}+\Phi\left(h_{\text {min }}\right) \\
\text { or }, \cos \theta & =1+\frac{\Phi\left(h_{\text {min }}\right)}{\gamma_{l g}}
\end{aligned}
$$

Here we note that the slope of $\Phi_{e l}(h)$, and thus the disjoining pressure $\Pi_{e l}(h)$, is the same at $h=h_{\min }$ and $h \rightarrow \infty$, which allows the two films of different thickness to coexist.

Figure 2.11c shows an asymptotically attractive concave potential. This scenario is analogous to Figure $2.11 \mathrm{~b}$, with $h_{\min }=0$. Part of the film dries up $(h=0)$, and part of it forms a macroscopic droplet $(h \rightarrow \infty)$. We thus 


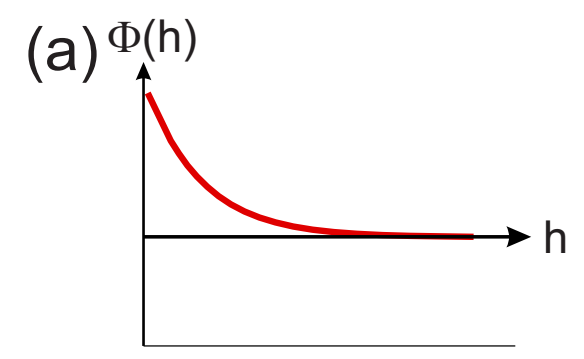

\section{Complete Wetting (CW)}

(b)
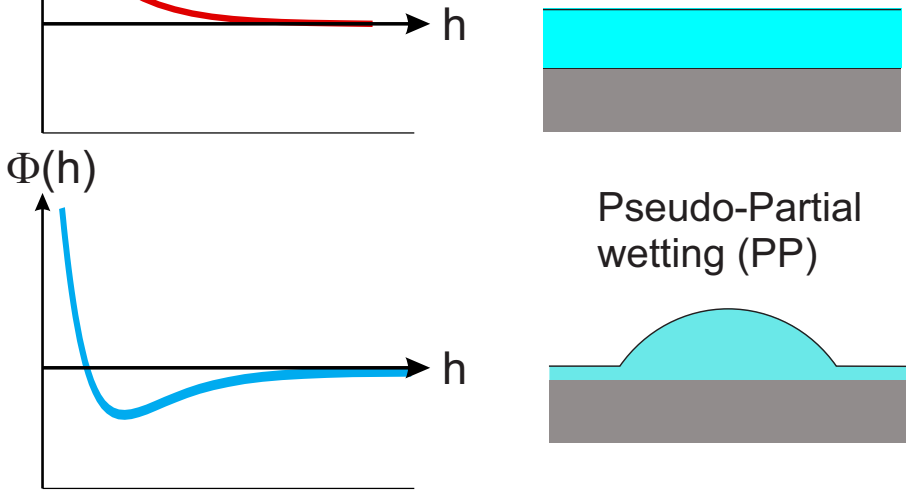

\section{Pseudo-Partial wetting (PP)}
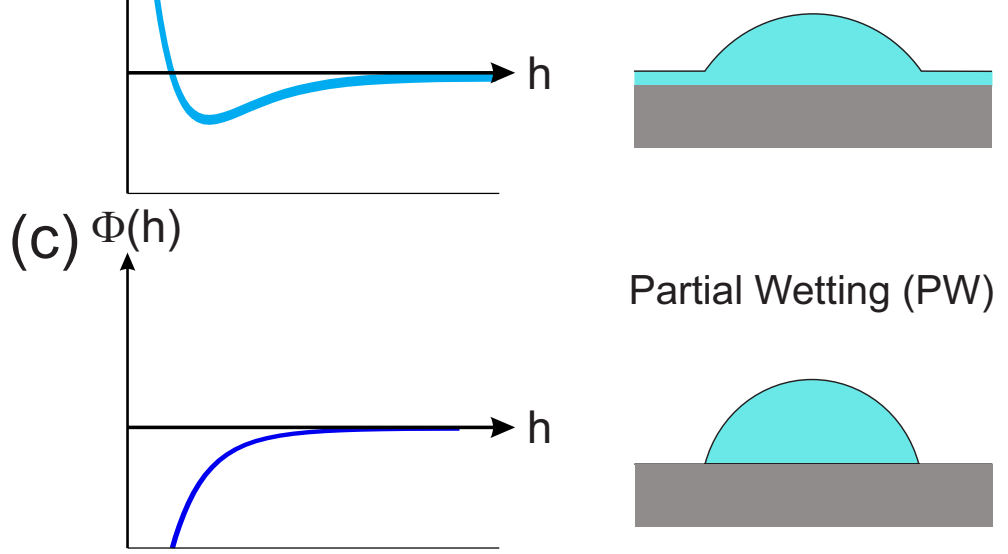

\section{Partial Wetting (PW)}
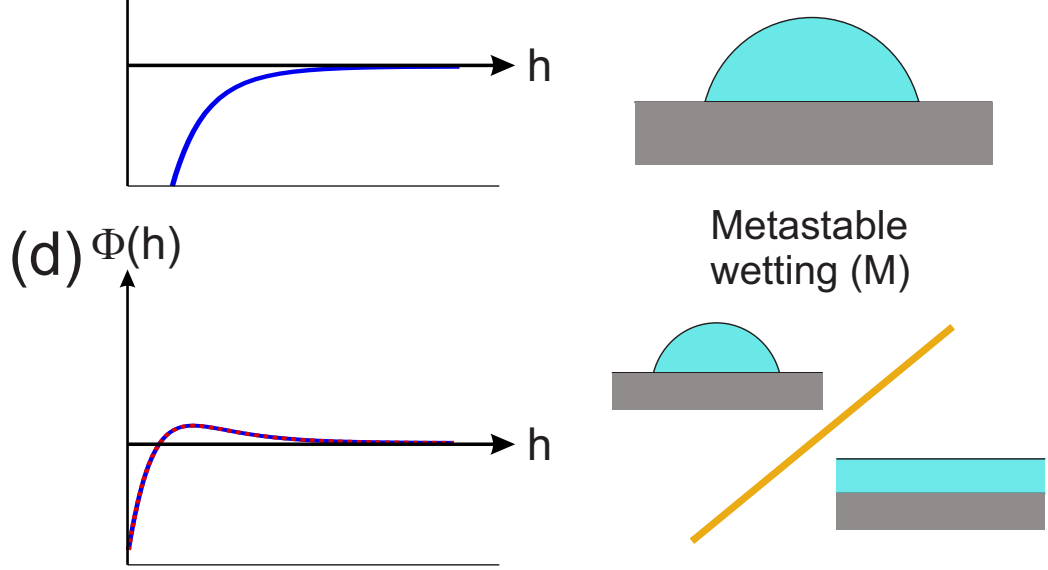

Figure 2.11: Four different types of interface potential $\Phi(h)$ along with corresponding wetting configurations.

obtain a partial wetting (PW) configuration. Finally, we consider the interface potential in Figure 2.11d. which exhibits a long-range repulsion and a short-range attraction. The associated wetting configuration is metastable. Depending on its initial thickness, a film will evolve into a completely wetting layer or a partially wetting droplet. 


\subsubsection{Wetting Transition}

A 'wetting transition' is a transition between the various wetting configurations we have explained in the last section, especially between complete and partial wetting states, which can be achieved either by a change of temperature or by a change of chemical potential $\mu$. For example, if there is a partially wetting liquid drop in our solid/gas system, and the temperature is changed until the wetting temperature $T_{w}$ is achieved and the liquid phase forms a thick wetting film co-existing between the solid and the gas phases, then $T_{w}$ is called the transition temperature. This temperature, obviously, depends on the critical point of co-existence between the liquid and the gas phase. In this case, we are often interested in the thickness of the wetting film, or more precisely, the manner in which the thickness of the liquid film changes upon transition. If there is an abrupt (discontinuous) change in the film thickness as the transition occurs, that means there is a discontinuity in the first derivative of the surface free energy (the grand canonical potential $\Omega$ ). This transition is then called a first order wetting transition. However, if there is a gradual change in the film thickness (no discontinuity in the first derivative of the surface free energy), then it is called a continuous wetting transition.

We have referred to wetting films of various thickness in our picture of adsorption induced wetting, pointing out the difference between microscopically thin films and macroscopically thin films. This implies that a threshold must exist between the two and the wetting transition is exclusively related to this threshold/critical film thickness $h_{c}$. However, it is only natural that sometimes the change in the film thickness could be significant without crossing this threshold. We will notice in our various experiments real examples of such cases. In this case, the transition is not between 'complete' (CW) and 'partial' (PW) wetting, but could be between a partial (PW) wetting and a pseudo-partial (PP) or Metastable (M) wetting. In accordance with the changes in the wetting films, such transitions are often referred to as thick to thin wetting transitions $[16,17]$.

\subsection{Surfactants}

Before closing this chapter, our final topic to introduce will be surfactants. Throughout this chapter, we have discussed standard molecules and ionic solutes which are adsorbed at an interface and gradually attain a thermody- 
namic equilibrium. However, in reality, most surface-active solute molecules are larger molecules, known as surfactants, and behave in a different manner. These molecules are amphiphilic molecules, containing a hydrophilic polar head group as well as a hydrophobic non-polar tail. As a result, the polar head shows affinity for an aqueous phase, while the non-polar tail prefers to remain in air or oil. The driving mechanism for surfactant adsorption is the loss of entropy for the molecules. Obviously, the surfactants are very fond of interfaces because of this simultaneous preference for oil/air and water. Once the surfactants are adsorbed at the interface, the surface tension decreases until it arrives an equilibrium value.

For reasons explained in Chapter 1 , we have chosen fatty acids (R-COOH) as representative polar components/surfactants in our experiments. Fatty acids of various aliphatic chain lengths are used and, in particular, only non-branched species have been used throughout this thesis. Increasing hydrophobicity of the tail implies stronger affinity for the interface, hence increasing the aliphatic chain length leads to stronger adsorption. At the same time, increasing the chain length reduces the solubility of the surfactant, forming micelles above a critical concentration ( $\mathrm{cmc}$ ). We will verify the consequences of these in our wetting experiments in Chapter 7.

\subsection{Concluding Remarks}

We have revisited the basic concepts of wetting and adsorption in this chapter. We first discussed the macroscopic concepts of surface tension, Laplace pressure and wetting, since a significant part of our experiments will cover macroscopic wetting trends. But in order to answer many questions stemming from our macroscopic observations, we will be required to delve into the phenomena at colloidal and microscopic regime, such as adsorption, electrostatic interaction, dispersion interaction among others. Wetting will be the central theme of this thesis, and after revising the relevant concepts and background of wetting, we now proceed to investigate some interesting systems, both experimentally and analytically. 


\section{Bibliography}

[1] P.G. de Gennes, F. Brochard-Wyart, and D. Quere. Capillarity and Wetting Phenomena. Springer, New York, 2004.

[2] J. Lyklema. Fundamentals of Interface and Collloid Science, Volume III : Liquid-Fluid Interfaces. Academic Press, London, 2000.

[3] T. Young. An essay on the cohesion of fluids. Philosophical Transactions of the Royal Society of London, 95:65-87, 1805.

[4] A. M. Brzozowska, F. Mugele, and M. H. G. Duits. Stability and interactions in mixed monolayers of fatty acid derivatives on artificial sea water. Colloids and Surfaces A: Physicochemical and Engineering Aspects, 433:200-211, 2013.

[5] A. W. Adamson. Physical chemistry of surfaces. A Wiley-Interscience publication. Wiley, 1990.

[6] H. J. Butt, K. Graf, and M. Kappl. Physics and Chemistry of Interfaces. Physics textbook. Wiley, Weinheim, 2003.

[7] I. Langmuir. Oil lenses on water and the nature of monomolecular expanded films. The Journal of Chemical Physics, 1(11):756-776, 1933.

[8] C. Chang and E. I. Franses. Adsorption dynamics of surfactants at the air/water interface: a critical review of mathematical models, data, and mechanisms. Colloids and Surfaces A: Physicochemical and Engineering Aspects, 100:1-45, 1995.

[9] A. F. H. Ward and L. Tordai. Time dependence of boundary tensions of solutions i. the role of diffusion in time effects. The Journal of Chemical Physics, 14(7):453-461, 1946.

[10] B. V. Deryagin and N. V. Churaev. Structure of water in thin layers. Langmuir, 3(5):607-612, 1987. 
[11] R. B. Schoch, J. Han, and P. Renaud. Transport phenomena in nanofluidics. Reviews of Modern Physics, 80(3):839-883, 2008.

[12] J. N. Israelachvili. Intermolecular and Surface Forces: Revised Third Edition. Intermolecular and Surface Forces. Elsevier Science, 2011.

[13] S. Loh and S. P. Jarvis. Visualization of ion distribution at the micaelectrolyte interface. Langmuir, 26(12):9176-9178, 2010.

[14] J.I. Kilpatrick, S-H. Loh, and S.P. Jarvis. Directly probing the effects of ions on hydration forces at interfaces. J. Am. Chem. Soc., 135:2628, 2013.

[15] W. B. Russel, D. A. Saville, and W. R. Schowalter. Colloidal Dispersions. Cambridge Monographs on Mechanics. Cambridge University Press, 1992.

[16] D. Bonn and D. Ross. Wetting transitions. Reports on Progress in Physics, 64(9):1085, 2001.

[17] D. Bonn, J. Eggers, J. Indekeu, J. Meunier, and E. Rolley. Wetting and spreading. Reviews of Modern Physics, 81(2):739-805, 2009.

[18] J. Lyklema. Fundamentals of Interface and Collloid Science, Volume I : Fundamentals. Academic Press, London, 2000. 


\section{CHAPTER 3}

\section{Experimental Methods}

\section{Abstract}

We discuss the standard experimental methods used for investigating wettability, wetting transition, interfacial properties such as surface tension, interfacial tension, surface charge. The methods for measuring the thickness of a molecularly thin wetting film are also described. Most importantly, in this chapter we introduce an extension of existing goniometry methods in order to measure relative trends in small contact angles. 


\subsection{Introduction}

In this chapter, we describe the various techniques used in our experiments such as optical goniometry for determining contact angles on a solid substrate, the pendant drop method for measuring interfacial tension (IFT) at oil-water interfaces, imaging ellipsometry for measuring the thickness of nanometer-scale wetting films and the streaming potential technique for measuring $\zeta$-potential and surface charge at an interface. We will see in the following chapters that contact angles on mica in alkanes are often $<10^{\circ}$. Normally, contact angles in the range of a few degrees are quite challenging to measure using the goniometry method. We have performed extra measurements to set the sensitivity and precision of the goniometer in a consistent manner which allows us to measure all the relative trends in these small contact angles. In this chapter, we will describe the methodology for these additional measurements.

\subsection{Contact Angle Goniometry}

\subsubsection{Precision of Goniometry Measurements}

We use a commercial goniometer (OCA 20L, Dataphysics Instruments GmBH, Germany) for measuring contact angles during wetting experiments. The measurement is based on the sessile-drop method using aqueous drops with a volume of $2 \mu \mathrm{l}$ placed on the substrate. The accuracy in the drop-volume is measured to be within $\pm 0.2 \mu \mathrm{l}$, which is calibrated with a micropipette. The contact angle of the drop is extracted from video snapshots using the tangent-fitting method as well as the circle-fitting method from the data analysis software (SCA 22) provided with the instrument. Circle fitting works best for sessile drops which are not deformed by gravity, while tangent fitting does not have any limitations for a drop deformed by gravity. However, tangent fitting becomes increasingly difficult for contact angles smaller than $10^{\circ}$, since the software can hardly distinguish between the reference baseline and the drop-profile. We have used both methods separately, in order to verify the values obtained from one of these methods, by comparing them with the values obtained from the other method. The minimum contact angle that can be determined on reflective surfaces is approximately $2^{\circ}$, because of the limitations of the fitting methods. It is usually argued that contact angles in the range of a few degrees are quite challenging to mea- 
sure using this macroscopic goniometry method, and the argument holds because of the reasons explained above. Nonetheless, we have followed a set of rigorous protocols and used consistent procedures in order to establish accurate relative trends in our measurement of small contact angles. These procedures are described in the following sections.

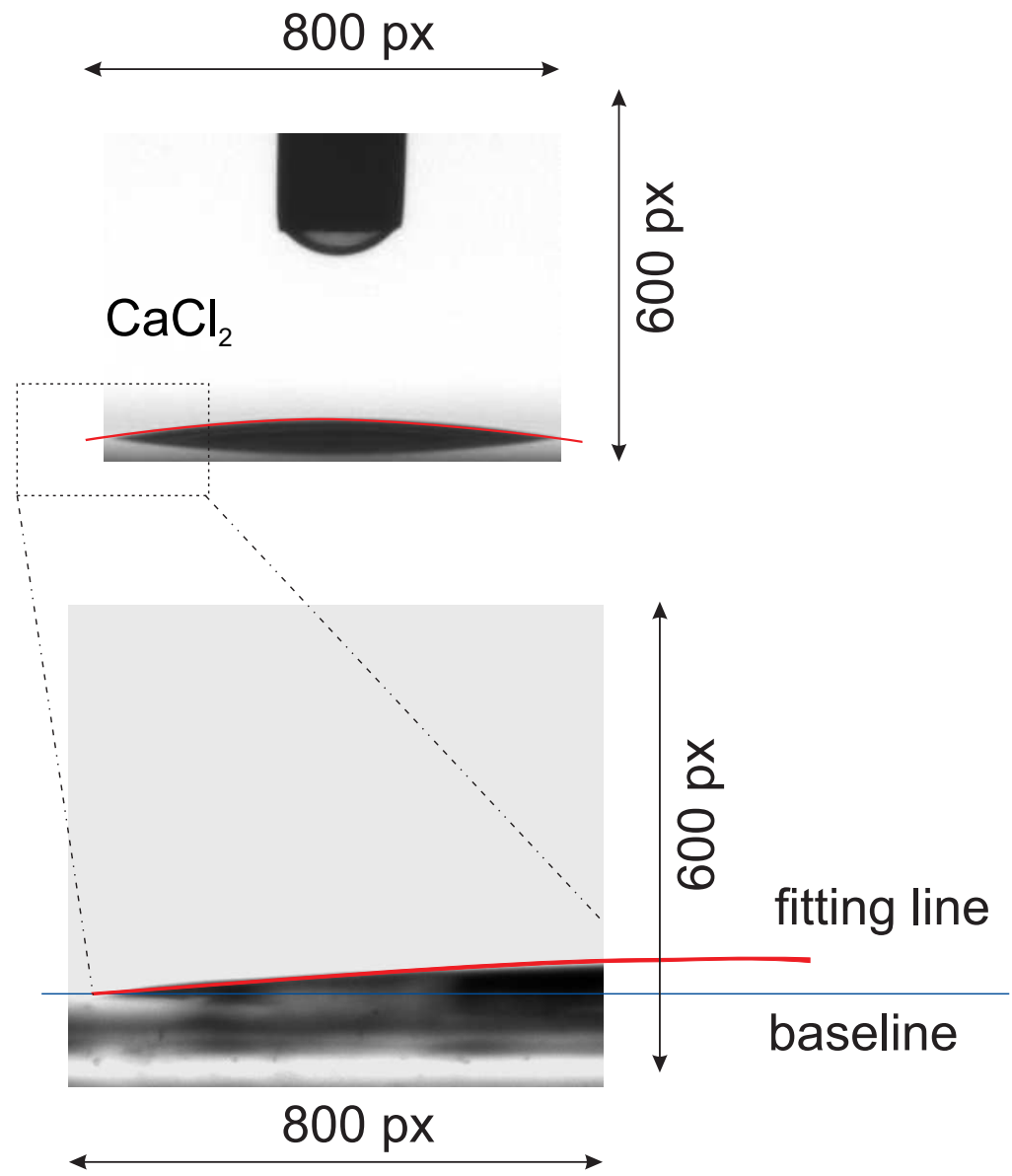

Figure 3.1: Pixel distribution for a sample contact angle measurement with a $1 \mathrm{M} \mathrm{CaCl}{ }_{2}$ drop on mica in decane. Enlarged view of one edge of the droplet is used during tangent/circle fitting, in order to maximize the pixel information around the contact line. 


\subsubsection{Magnification}

The contact angle goniometry method is effectively fitting a tangent/circle to the profile of a drop. Therefore the accuracy greatly depends on the ability of the SCA 22 software to capture the drop profile with respect to the background. Therefore, it is crucial for the software to be able to distinguish between the actual drop and its reflection on the solid substrate (which is done with a reference baseline). Thus, the profile recognition depends on the pixel information available from the image/series of images. The video of the droplet is captured at $2 \times$ magnification and has $800 \times 600$ pixels (Figure 3.1). However, in order to increase the pixel information around the region of our interest, i.e., the 3-phase contact line, we zoom in (3.5x magnification) at one edge of the drop. As a result, the outline of the drop-profile is easily identified by SCA 22 in backdrop of the ambient phase.

\subsubsection{Illumination}

Illumination plays a very important role in our precision measurements, since the illumination of the system determines the sharpness of the edge between the aqueous drop and the background. The illuminating lamp (intrinsic part of the goniometer) is always kept constant at half of its maximum strength and the illumination parameters are varied using the 'Framegrabber Preferences' (inherent in SCA 22). All our measurements are carried out with the following set of illumination parameters: Brightness-125, contrast215 and Gamma (illumination)-100. As shown in Figure 3.2, for a reduced illumination, the fitting circle changes, providing a higher contact angle (by $\sim 2^{\circ}$ ). An increased illumination, on the other hand, provides a lower contact angle. Hence, we conclude that the precision of the contact angle measurement is $\sim 2^{\circ}$ for varying illumination.

\subsubsection{Baseline}

Once the favorable illumination is set, we focus on the baseline detection for the drops. During the analysis of the captured video, there are 2 reference lines used by the software, the first is placed at the top plane of the substrate automatically by SCA 22 (we call it the reference baseline) and the other is placed in the ambient phase, without intersecting the image of the drop phase (Figure 3.3). For correct detection of the contact angle, it is crucial that the reference baseline is exactly in between the drop and its reflection. In other words, the total number of pixels used in the profile-fitting analysis is 


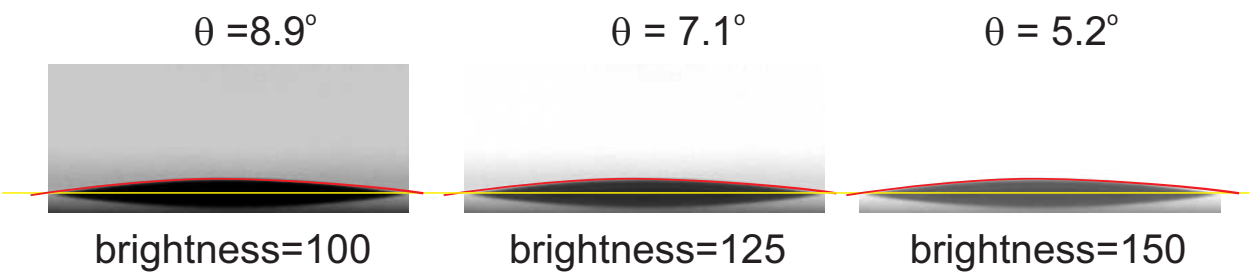

Figure 3.2: Comparison of contact angle fit by SCA 22, based on various brightness settings. All our experiments are carried out at brightness 125, while for lower (100) and higher (150) brightness values, we obtain higher and lower contact angles, respectively. In all the images, contrast (215) and Gammaillumination (100) are constant, the yellow line is the reference baseline while the red curve is section of the circle fitting the drop-profile for contact angle extraction.

the set of pixels between the two reference lines. In order to fit the best tangent/circle to the drop profile, as many pixels from this region as possible should be available to the software.

For our experiments, we always keep the reference baseline untouched, which means that the position of the baseline is solely determined by the analyzing software itself. As shown in Figure 3.3, if the baseline is not precisely

$$
\theta=6.2^{\circ}
$$

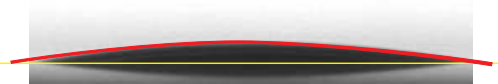

baseline 20 px lower

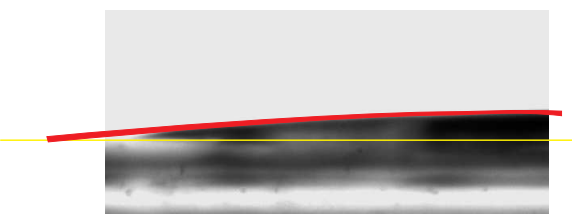

$\theta=7.1^{\circ}$
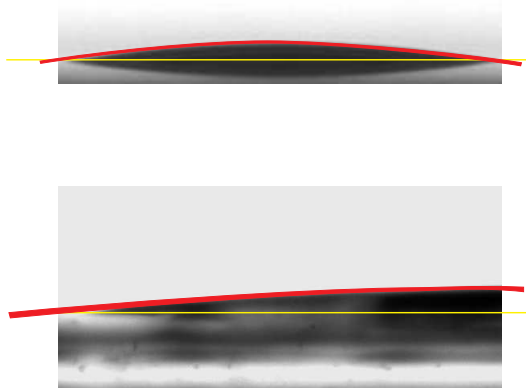

Figure 3.3: Contact angle fit when the baseline is lowered (top left, with enlarged view shown bottom left) by 20 pixels in compared to the automatic baseline detection by SCA 22 (top right, with enlarged view shown bottom right); In this case, the change of baseline leads to almost an $1^{\circ}$ error in the contact angle fitting. 
at the substrate-ambient interface, then the value of the measured contact angle varies. It is possible to visibly determine the actual baseline (substrateaqueous drop interface) to within \pm 5 pixels. However, if we drag the reference line to a position higher than the actual baseline, then the number of available pixels is reduced. Lowering the reference line increases the number of pixels, but at the expense of accuracy. Hence, for all the experiments performed, the stage of the goniometer is moved in the vertical direction (upward or downward) until it coincides with the software baseline. This precaution brings down the error of measurement to $\pm 1^{\circ}$.

\subsubsection{Spherical Cap Fit}

We used the analytical expression of a spherical cap in order to validate the accuracy of the contact angle calculated by SCA 22. As shown in Figure 3.4 , the base radius of the droplet can be measured from SCA 22. Also our ellipsometry experiments capture the base profile of the deposited drop. The base of the drop is an irregular profile, not a circular one, but it's possible to calculate an approximate base diameter from this profile using SCA 22. Once we have the base radius (r) for the drop, we can calculate the contact angle using the following relation:

$$
V_{\text {cap }}=\frac{1}{3} \pi \frac{r^{3}}{\sin ^{3} \theta}\left(2-\cos \theta+\cos ^{3} \theta\right)
$$

where $V_{\text {cap }}$ is the volume of the deposited droplet (in all our experiments, $2 \mu \mathrm{l})$. Once $\theta$ is calculated it can be compared with the drop profile-fit $\theta_{\text {fit }}$, and the difference is a maximum of $20 \%$ (for contact angles $<10^{\circ}$ ), which we accept to be within our error of measurement. An example of this comparison of calculated and measured contact angle is shown in Figure 3.4.

$$
\theta=7.1^{\circ}
$$$$
\text { calculated } \theta=7.6^{\circ}
$$
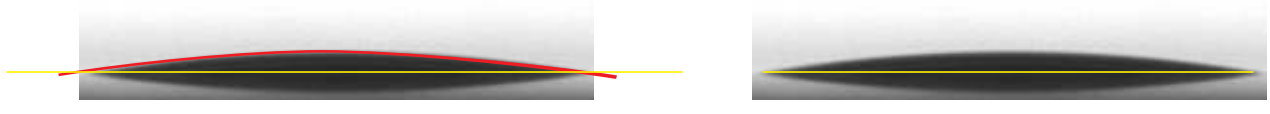

measured $\mathrm{BD}=8.2 \mathrm{~mm}$

Figure 3.4: Comparison of contact angle profile fit from SCA 22 (on the left) and a value obtained from a spherical cap calculation (right). The spherical cap calculation is based upon the drop base diameter obtained from SCA 22. 


\subsubsection{Reproducibility}

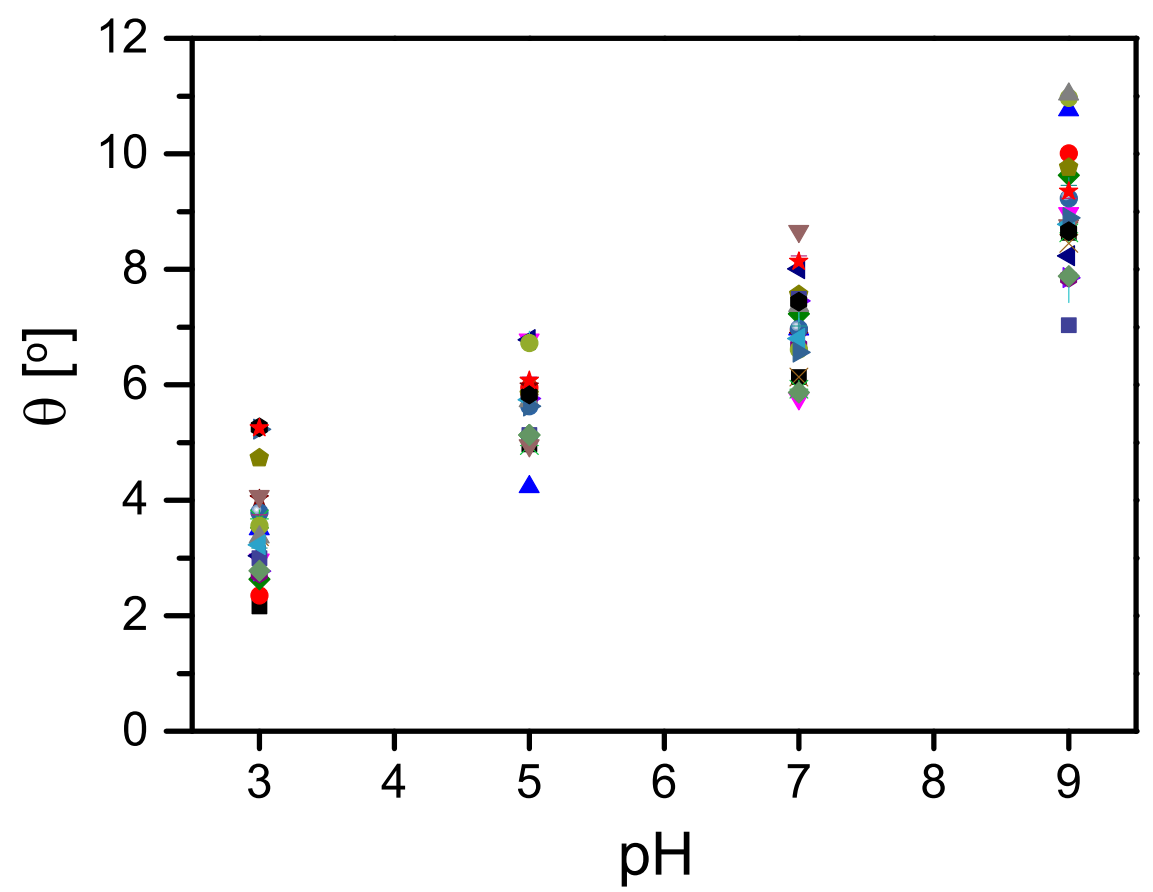

Figure 3.5: Contact angle of $1 \mathrm{M} \mathrm{CaCl}_{2}$ on mica in ambient decane with the aqueous salt solution at various $\mathrm{pH}: 3,5,7$ and 9 (various symbols and colors). 25 datapoints are shown for every $\mathrm{pH}$, based on which the mean and the standard deviation have been calculated.

We have performed 25 independent measurements under identical conditions (room temperature, humidity) for every datapoint in the contact angle goniometry experiments. Each mica substrate of dimension $3.5 \mathrm{~cm} \times 1 \mathrm{~cm}$ is cut from the mica sheet (B\&M Mica Company, USA) and cleaved under oil to prepare a pristine surface. In order to obtain a general contact angle trend irrespective of a mica substrate, at least 5 different mica substrates are used for depositing the set of 25 drops of a certain salt concentration and $\mathrm{pH}$. Throughout the thesis, we will represent the mean of these measurements with the standard deviation of the dataset shown: 


$$
S D=\sqrt{\frac{1}{N} \sum_{1}^{N}\left(x_{i}-\bar{x}\right)^{2}}
$$

where, $x_{i}$ is the individual experimental measurement and $x$ is the mean of 25 measurements. Figure 3.5 shows an example of a complete dataset collected for contact angle of $1 \mathrm{M} \mathrm{CaCl}_{2}$ solution drops on mica in ambient decane at 4 different $\mathrm{pH}$ values 3, 5, 7 and 9 .

\subsubsection{Final Remarks}

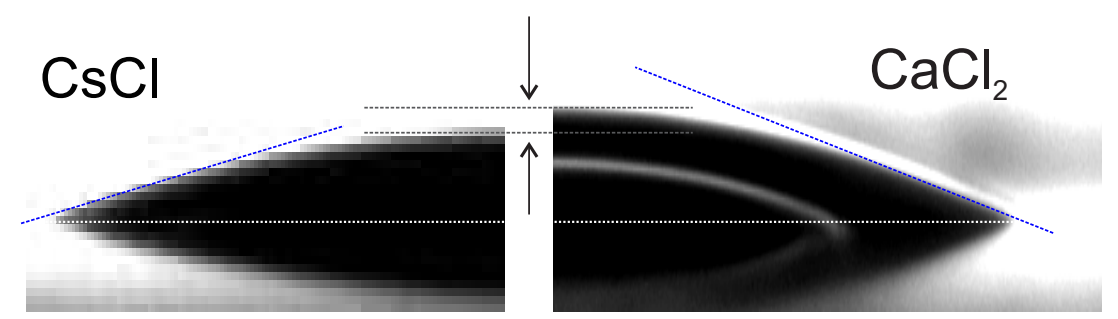

Figure 3.6: Precision of contact angle measurement using optical goniometry: For drops of $1 \mathrm{M} \mathrm{CsCl}\left(\theta \sim 7^{\circ}\right)$ and $1 \mathrm{M} \mathrm{CaCl}_{2}\left(\theta \sim 9^{\circ}\right)$ solution with same base diameter, the difference in contact angle is shown. Both the images are stretched to $200 \%$ in vertical direction in order to highlight the difference in drop height.

We have described the various protocols which we have followed, in order to measure contact angles below $10^{\circ}$. Although it is still extremely challenging to determine such small contact angles with a high absolute accuracy, our procedure allows us to obtain a reliable relative trend in the wetting experiments. We have shown an example in Figure 3.6, where a 1 $\mathrm{M} \mathrm{CsCl}$ and a $1 \mathrm{M} \mathrm{CaCl}_{2}$ (both at $\mathrm{pH} 9$, placed on the same mica substrate in ambient decane) are compared with each other. It is clear from the height of the drops as well as from the corresponding tangent fitting (blue) lines, that the contact angle for the $\mathrm{CaCl}_{2}$ drop is larger than that of the $\mathrm{CsCl}$ drop. Later in Chapter 6, we discuss the difference in the wetting of mica (in alkanes) by $\mathrm{CsCl}$ and $\mathrm{CaCl}_{2}$, and this difference in contact angle is $\sim 2^{\circ}$. We also demonstrate a certain degree of reproducibility in the contact angles following these additional protocols. It is important to note the minimum value of the contact angle that can be measured with optical goniometry. After 
following these procedures, $2^{\circ}$ is the lowest value of contact angle which we can measure. For a drop with a contact angle lower than $2^{\circ}$, we will 'assign' the corresponding contact angle $\theta$ to be $0^{\circ}$.

\subsection{Pendant Drop Measurements}

Before placing the aqueous drops on the substrates, pendant drop measurements are performed to determine the oil/water interfacial tension (IFT). The IFT trend is crucial in wetting experiments, since for a system without any surfactant the oil/water interfacial tension should remain constant. On the other hand, in a system with surfactants, the amount of decrease in IFT needs to be noted for analyzing the changes in contact angle. In a pendant drop measurement, we rotate the camera in OCA by $90^{\circ}$, so that the vertical needle is captured as a horizontal needle from right of the video. This is done in order to maximize the number of pixels (800 pixels) during the profile-fitting. Such a pendant drop of $30 \mu \mathrm{l}$ volume is created and suspended from the needle (Precision Tips, Nordson EFD, USA, ID $0.82 \mathrm{~mm}$ ), as shown in Figure 3.7. Because of the interfacial tension between oil and water phases, there is a build-up of Laplace pressure inside the drop:

$$
\Delta p=\gamma\left(\frac{1}{r_{1}}+\frac{1}{r_{2}}\right)
$$
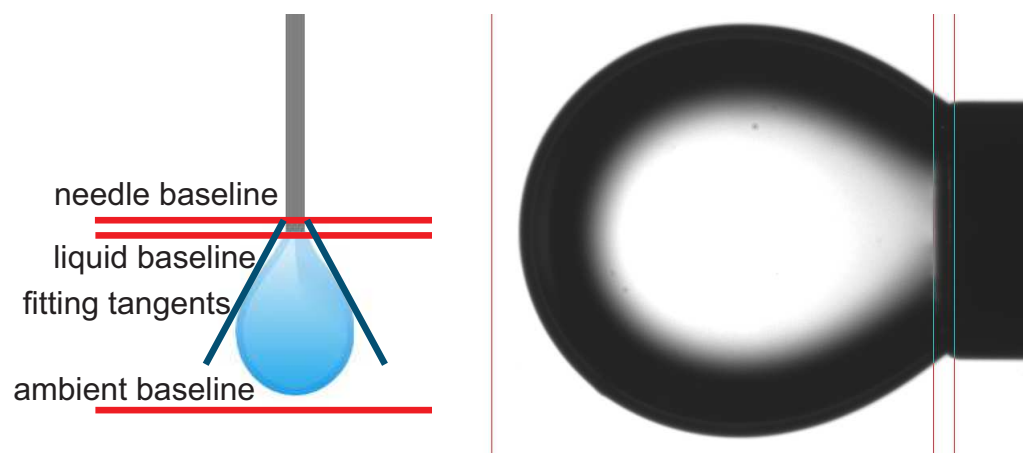

Figure 3.7: Left, schematic of the pendant drop method (Figure adapted from [1]) with the various reference baselines and fitting tangents. Right, a typical example of a pendant drop in our experiments, $1 \mathrm{M} \mathrm{CaCl}_{2}$ solution at $\mathrm{pH} 7$, in ambient decane for measurement of water-decane interfacial tension (IFT). 
where, $r_{1}$ and $r_{2}$ are the radii of curvature of the pendant drop. Ideally, the drop should be spherical under the influence of interfacial tension only. However, there is also the effect of deformation of the drop caused by gravity, creating a 'pear' shaped drop. The IFT is measured by SCA 22 using drop shape analysis from the shadow image of the pendant drop.

\subsection{Ellipsometry}

In-situ thickness measurements of ultrathin wetting films co-existing with a spreading drop were performed by imaging ellipsometry at room temperature $\left(20 \pm 1^{\circ} \mathrm{C}\right)$ using a nulling ellipsometer at a single wavelength of 658 $\mathrm{nm}\left(\mathrm{EP}^{3}\right.$ Accurion $\mathrm{GmbH}$, Germany). The reflected light from the surface is focused with a $2 x$ objective and amplified by a beam expander leading to a $2 \times 2 \mathrm{~mm}^{2}$ field of view and enabling a lateral resolution of $2 \mu \mathrm{m}$. If we use a coordinate system defined by the direction of propagation and the plane of incidence, an electric field vector $\mathbf{E}$ defines the polarization of the light wave and can be decomposed into two perpendicular components $\mathrm{E}_{p}$ and $\mathrm{E}_{s}$ respectively parallel and perpendicular to the plane of incidence. Therefore, the ratio $\rho$ of $\mathrm{p}$ and s components of the reflection matrix can be described by: $\rho=\frac{E_{\text {out }, p}}{E_{\text {out }, s}} \frac{E_{i n, s}}{E_{i n, p}}=\tan \left(\psi_{\text {ellip }}\right) \exp \left(i \delta_{\text {ellip }}\right)$, where $0<\psi_{\text {ellip }}<90^{\circ}$ and $0<$ $\delta_{\text {ellip }}<360^{\circ}$ are the ellipsometric angles while $\mathrm{E}_{\text {in } / \text { out }}$ denotes the electric field of the incoming/outgoing light. Moreover, $\tan \left(\psi_{\text {ellip }}\right)=\frac{E_{p}}{E_{s}}$ represents the relative amplitude attenuation and $\delta_{\text {ellip }}=\left[E_{p}\right]-\left[E_{s}\right]$ is the phase lag of the $\mathrm{p}$ and $\mathrm{s}$ linearly polarized components upon reflection. We performed ellipsometer experiments using the Multiple-angle-of-incidence ellipsometry (MAIE) method which involves measurement of $\delta_{\text {ellip }}$ and $\psi_{\text {ellip }}$ values as a function of the angle of incidence (AOI), thus this information allows us to determine the dielectric function of the material studied. The dependence on the incidence angle of the ellipsometric angles was measured and averaged using a 4-zone nulling scheme. The analysis of the ellipsometric angles, $\delta_{\text {ellip }}$ and $\psi_{\text {ellip }}$ was done assuming that the surface can be described by the corresponding optical model (Drude model). The accuracy of $\delta_{\text {ellip }}$ and $\psi_{\text {ellip }}$ is better than \pm 0.1 and \pm 0.05 degrees, respectively.

The ellipsometer is equipped with custom-built quartz tubes attached to both the source (laser) and the detector arm to enable measurements under liquid (in this case, decane) at variable angles of incidence (AOI). The intensity of the reflected light is minimized by a $90^{\circ}$ rotation of the anal- 
yser and the ellipsometric angles $\delta_{\text {ellip }}$ and $\psi_{\text {ellip }}$ are mapped. The Drude model with standard Fresnel coefficients is used to relate the ellipticity $\rho=$ $f\left(\delta_{\text {ellip }}, \psi_{\text {ellip }}\right)$ to determine the thickness of the thin water layer. In the case of mica, the bottom side of the substrate is roughened and coated with an index matched epoxy resin to suppress interference.

\subsection{Streaming Potential Measurements}

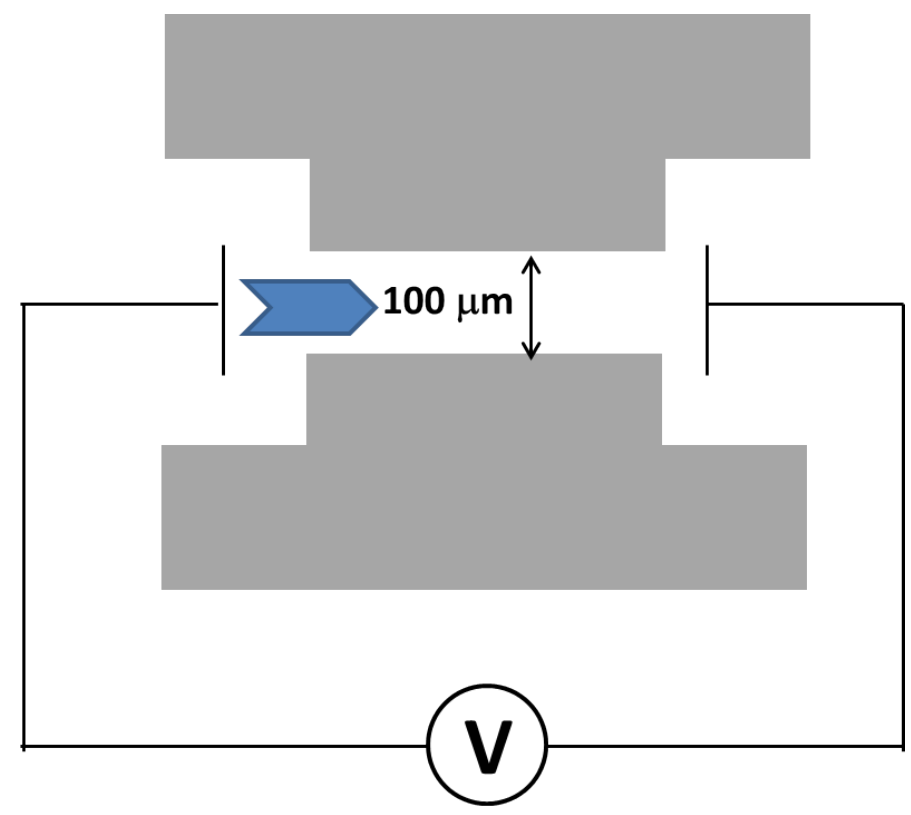

Figure 3.8: A schematic of the cell and circuit for streaming potential measurements in ZetaCAD. The arrow denotes the direction of flow induced by pressure drop $\Delta \mathrm{P}$.

In order to determine the surface charge/potential of solid/water and oil/water interfaces, we performed streaming potential measurements using a ZetaCAD (CAD Instruments, France). The measurement cell consists of the two solid surface (material under investigation, $50 \mathrm{~mm} \times 30 \mathrm{~mm}$ ) at a separation of $100 \mu \mathrm{m}$. The liquid phase is forced to flow in this cell between the substrates by a pressure difference $\Delta \mathrm{P}$ (Figure 3.8). This flow of the charged species (present within the liquid phase at a certain $\mathrm{pH}$ and ionic strength) results in a streaming potential $\left(\mathrm{U}_{s t r}\right)$. We have measured these 
streaming potentials at different pressure drop values $(\Delta \mathrm{p})$. Streaming potentials are converted to $\zeta$-potentials using the standard Smoluchowski formula, $\zeta=\frac{\lambda \mu U_{s t r}}{\epsilon \epsilon_{0} P}$. ( $\mu$ : viscosity; $\epsilon$ : permittivity of liquid; $\epsilon_{0}$ : absolute permittivity; $\lambda$ : conductivity of the liquid). The pressure is ramped between -500 to +500 mbar with a step width of 10 mbar or less. While performing the experiment, it is assumed that the flow within the cell (i.e. between the mica substrates) is laminar irrespective of the differential pressure and the separation between the walls is large compared to the double layer thickness, which is fulfilled since Debye length $k_{D}^{-1} \sim 1-10 \mathrm{~nm}$ while the channel height is $\sim 100 \mu \mathrm{m}$.

We have calibrated the instrument with measurements of $\zeta$-potential at silica-water interfaces. Figure 3.9 shows the calibration data, where various concentrations of $\mathrm{NaCl}$ solution are used between silica substrates. The data has been compared with the silica $\zeta$-potential values in literature $[2,3]$.

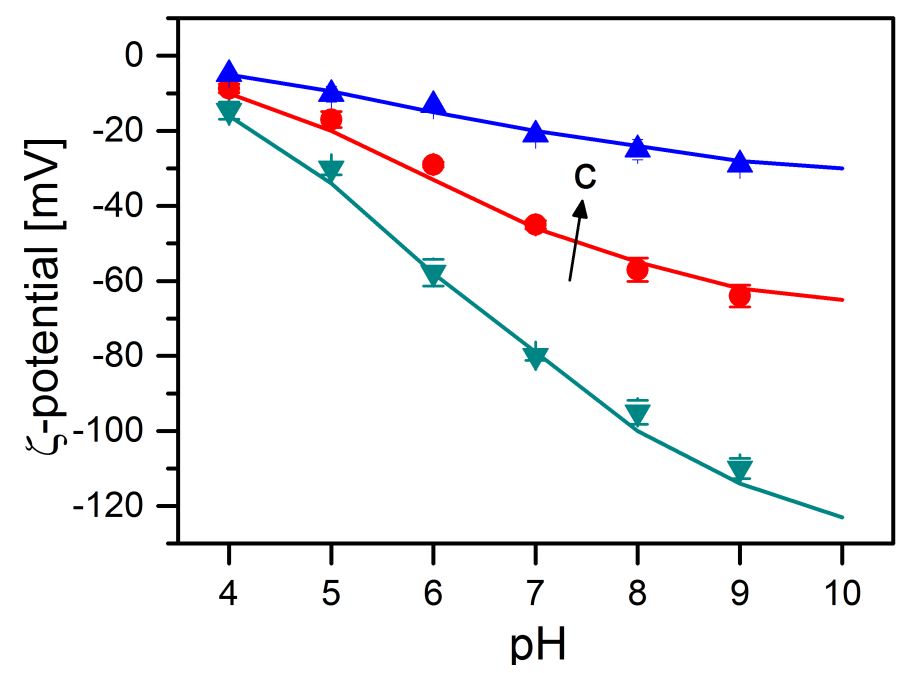

Figure 3.9: Calibration measurement $\zeta$-potential at silica-water interface vs $\mathrm{pH}$ of aqueous $\mathrm{NaCl}$ solution at various salt concentrations: $1 \mathrm{mM}$ (dark cyan downward triangles), $10 \mathrm{mM}$ (red circles) and $100 \mathrm{mM}$ (blue triangles). The $\mathrm{pH}$ is varied by adding $0.01 \mathrm{M} \mathrm{KOH}$ or $\mathrm{HNO}_{3}$ solutions to the salt solution prepared at neutral $\mathrm{pH}$. The symbols are average of 5 independent experiments under identical conditions with the standard error shown. The straight lines are reference values w.r.t. which the experimental datapoints are compared. 


\subsection{Langmuir Blodgett Trough}

The organic layer on substrates is prepared using an automated Langmuir Blodgett trough (NIMA technology, model 1212D1). Room temperature (22 $\pm 0.5^{\circ} \mathrm{C}$ ) and humidity (40\%) were monitored and kept at controlled values throughout the layer deposition experiments. In order to avoid vibration from surroundings and ensure stable conditions during the transfer, the trough is placed on a floating table. To prevent any contamination arising from the dust in the room, the trough is housed in a home-built laminar flow chamber, where the airflow is switched off during the entire deposition process. Before the deposition process, the trough was cleaned extensively using pure water, ethanol, isopropyl alcohol and finally with chloroform in order to remove any trace stearic acid. The subphase, which has been prepared prior to the cleaning, is then poured into the trough. In order to ensure a clean interface, various impurities were removed from the suphase-air interface by suction (Neolab vacuum system). The surface of the subphase is assumed to be clean (i.e., free of any impurity or extra surfactant) if the change in surface pressure of the subphase prior to spreading the organic phase, (stearic acid, henceforth referred to as SA) solution is $<0.1 \mathrm{mN} / \mathrm{m}$, when we carry out consecutive decompression and compression of the interface using the barriers on top of the subphase. Measurements of surface pressure are carried out with a Wilhelmy plate attached to the pressure sensor, where the last of the chain of plates (filter papers) is in contact with the uppermost layer of the interface.

The surface pressure and the subsequent deposition process is controlled with the NIMA 2.1 software provided with the the automated trough. Once the impurities are removed from the bare subphase, pre-cleaned substrates are submerged in the subphase. Subsequently, $50 \mu \mathrm{L}$ of SA solution $\left(\mathrm{CHCl}_{3}\right.$ as solvent) is evenly spread (using a Hamilton microsyringe) on the subphase and $\sim 30 \mathrm{~min}$ is allowed for the solvent to evaporate. At this stage, the SA molecules are distributed over an area of $500-700 \mathrm{~cm}^{2}$. Subsequently, we compress the subphase area following a pressure isotherm method, where the final target pressure is set as $30 \mathrm{mN} / \mathrm{m}$ and the compression speed is set at $10 \mathrm{~cm}^{2} / \mathrm{min}$. It is assumed that none of the SA molecules escapes the confined subphase area during the compression process. The Wilhelmy plate measures the in-situ surface pressure during this compression, and once the target pressure is reached, all the SA molecules are compressed within this area, with the substrates in between the barriers. 
The final stage of the deposition process is gradually lifting the submerged substrates out of the subphase, when the SA molecules get stuck to the substrate in a uniform monolayer. The lifting speed is set at $2 \mathrm{~mm} / \mathrm{min}$. This low value of the speed is governed by the consideration that complete drainage of water needs to take place during the deposition process in order to preserve the structure of the film. The quality of the deposited monolayer is verified using atomic force microscopy before performing contact angle measurements. 


\section{Bibliography}

[1] Kibron. Langmuir-blodgett troughs and surface tensiometers, 2015.

[2] A. Crespy, A. Bolève, and A. Revil. Influence of the Dukhin and Reynolds numbers on the apparent zeta potential of granular porous media. Journal of Colloid and Interface Science, 305(1):188-194, 2007.

[3] M. Wang and A. Revil. Electrochemical charge of silica surfaces at high ionic strength in narrow channels. Journal of Colloid and Interface Science, 343(1):381-386, 2010. 


\section{CHAPTER 4}

\section{Ion-induced Wetting Transition in Mineral/Water/Oil systems}

\section{Abstract}

In this chapter, we experimentally analyze ion adsorption at the solid-liquid interface leading to charge reversal, and how it causes wettability alteration for a model mineral-water-oil system. We investigate wettability alteration on a mica surface in ambient oil (various alkanes), as we change the cation in the aqueous salt solution from a monovalent to a divalent one. Along with varying the types of cations (and partially, also anions) in the salt, we vary their concentration as well as $p H$ in order to find the regime (of concentration and $\mathrm{pH}$ ) where the wetting transition takes place. We formulate an ion-adsorption based mechanism in a nm-scale thin wetting film with focus upon surface charge reversal caused by divalent cations on the mineral surface. We explore the properties of the thin wetting film and the surface charge information in mineral as well as oil interfaces in order to validate our propositions about charge reversal and wetting properties.

Parts of this chapter have been published as:

1. F. Mugele, B. Bera, A. Cavalli, I. Siretanu, A. Maestro, M. H.G. Duits, M. A. Cohen-Stuart, Dirk van den Ende, I. Stocker \& I. Collins. Ion adsorption-induced wetting transition in oilwater-mineral systems. Nature Scientific Reports, 5, DOI: 10.1038/srep10519 (2015).

Dr. A. Maestro is acknowledged for ellipsometry investigation and Dr. I. Siretanu is acknowledged for atomic resolution images of mica using AFM. 


\subsection{Introduction}

The relative wettability of oil and water on porous solids is crucial to many environmental and technological processes including imbibition, soil contamination/remediation, oil-water separation, and the recovery of crude oil from geological reservoirs $[1,2,3,4,5,6,7]$. Strong wettability of a porous matrix to one liquid generally implies stronger retention of that liquid and simultaneously easier displacement of the other. In standard 'water flooding' oil recovery, (sea) water is injected into the ground to displace oil from the porous rock, typically leading to a final recovery factor $<50 \%$. For decades, oil companies have explored adding chemicals such as surfactants and polymers to the injection water to improve the process $[8,9]$.

More recently, it was rediscovered that the efficiency can also be improved by reducing the salinity of the injection water[10], i.e. without adding expensive and potentially harmful chemicals, known as low salinity water flooding. Yet, reported increases in recovery vary substantially and the microscopic mechanisms responsible for the recovery increment remain debated $[9,11,12,13]$. A wide variety of mechanisms has been proposed to explain the effect, including the mobilization of fines, interfacial tension variations, multicomponent ion exchange, and double layer expansion [10,11, 12, 14], as we have already discussed in Chapter 1. Many of these mechanisms are interrelated making the rock more water wet, but evidence discriminating between them is scarce. The key challenge in identifying the reasons for the success of low salinity waterflooding lies in the intrinsic complexity of the system and the lack of direct access to its microscopic properties.

In this chapter, we experimentally investigate wettability alteration in a simplified model system representing the wetting situation in an oil-reservoir. We rationalize the observations by a mechanism of ion adsorption at the solid-liquid interface to charge reversal and from there to wettability alteration. We focus on the charge effects of ion-adsorption and experimentally investigate the wetting films and surface charges. Our results clarify many previous observations in core flooding experiments, including in particular the relevance of divalent cations, clay and $\mathrm{pH}$. 


\subsection{Experimental Methods}

\subsubsection{Materials}

Anhydrous decane ( $>99 \%$, Sigma Aldrich) is the oil phase in the experiments. It is passed five times through a vertical column of Alumina powder $\left(\mathrm{Al}_{2} \mathrm{O}_{3}\right.$, Sigma Aldrich, Puriss grade $\left.>98 \%\right)$ to remove surface-active impurities until the interfacial tension (IFT) is constant within $\pm 2 \mathrm{mN} / \mathrm{m}$ for at least 20 minutes. Decane-water IFT is measured using the Pendant Drop Method, and the usual value is $50 \pm 1 \mathrm{mN} / \mathrm{m}$. Ultrapure water (resistivity $18 \mathrm{M} \Omega$ ) for preparing salt solutions is obtained from Millipore (Synergy UV Instruments). We have prepared all inorganic salt (Sigma Aldrich) solutions with a concentration range of $1 \mathrm{mM}$ to $1 \mathrm{M}$ by adding the ultrapure water to carefully measured amount of the required salt. The $\mathrm{pH}$ of the solutions is adjusted between 2 and 10 using $\mathrm{HCl} / \mathrm{HNO}_{3}$ and $\mathrm{NaOH}(0.1 \mathrm{M}$, Sigma Aldrich). Muscovite mica (B\&M Mica Company Inc., USA; initial thickness $340 \mu \mathrm{m}$ ) and oxidized silicon wafers with an amorphous silicon oxide layer (thickness: $30 \mathrm{~nm}$ ) mimicking silica represents the solid rock surface. Mica sheets are cleaved inside the oil with scotch tape to obtain a pristine surface during the experiment. Silica surfaces are cleaned using a combination of Piranha solution (mixture of $30 \%$ conc. $\mathrm{H}_{2} \mathrm{O}_{2}$ and $70 \%$ conc. $\mathrm{H}_{2} \mathrm{SO}_{4}$ ) followed by extensive rinsing with ultrapure water and plasma treatment.

\subsubsection{Optical Goniometry}

The wetting of aqueous drops on mica/silica is characterized by equilibrium contact angles, using a commercial contact angle goniometer (OCA 20L, Dataphysics Instruments GmBH, Germany). The measurement is based on the sessile-drop method using aqueous drops of volume $2 \mu \mathrm{l}$ placed on the substrate. The accuracy in the drop-volume is measured to be within $\pm 0.2 \mu \mathrm{l}$. The contact angle of the drop is extracted from video snapshots using the tangent and circle-fitting data analysis software (SCA 22) provided with the instrument. Contact angles can be determined with a relative accuracy of $\pm 1^{\circ}$. The minimum contact angle that can be determined on reflective surfaces is $\sim 2^{\circ}$, because of the limitations of tangent/circle fitting method. All experimental datapoints are average of 25 independent measurements (drops) placed on at least 5 different solid substrates. Before placing the aqueous drops on the substrates, pendant drop measurements are performed to determine the oil/water interfacial tension (IFT). Normally, 
contact angles in the range of a few degrees are quite challenging to measure using goniometry method. But we have done extra measurements to set the sensitivity and precision of the goniometer at a scale, which allows us to measure all the relative trends in these small contact angles. It was not possible to perform advancing-receding measurements, since the receding contact angle for most of these small values is $0^{\circ}$. However, we measure the base diameter of our drops optically, and fit the contact angles with a spherical cap approximatrion for better accuracy. Various technical aspects of our goniometry measurements are discussed in detail in Chapter 3.

\subsubsection{Imaging Ellipsometry}

In-situ thickness measurements of ultrathin wetting films co-existing with a spreading drop were performed by imaging ellipsometry at room temperature $\left(20 \pm 1^{\circ} \mathrm{C}\right)$ using a nulling ellipsometer at a single wavelength of 658 $\mathrm{nm}$ ( $\mathrm{EP}^{3}$ Accurion $\mathrm{GmbH}$, Germany). The reflected light from the surface is focused with a $2 x$ objective and amplified by a beam expander leading to a $2 \times 2 \mathrm{~mm}^{2}$ field of view and enabling a lateral resolution of $2 \mu \mathrm{m}$. If we use a coordinate system defined by the direction of propagation and the plane of incidence, an electric field vector $\mathbf{E}$ might define the polarization of the light wave and can be decomposed into two perpendicular components $\mathrm{E}_{p}$ and $\mathrm{E}_{s}$ respectively parallel and perpendicular to the plane of incidence. Therefore, the ratio $\rho$ of $\mathrm{p}$ and s components of the reflection matrix can be described by: $\rho=\frac{E_{\text {out }, p}}{E_{\text {out }, s}} \frac{E_{\text {in }, s}}{E_{\text {in }, p}}=\tan \left(\psi_{\text {ellip }}\right) \exp \left(i \delta_{\text {ellip }}\right)$, where $0<\psi_{\text {ellip }}<90^{\circ}$ and $0<\delta_{\text {ellip }}<360^{\circ}$ are the ellipsometric angles while $\mathrm{E}_{\text {in } / \text { out }}$ denotes the electric field of the incoming/outgoing light. $\tan \left(\psi_{\text {ellip }}\right)=\frac{E_{p}}{E_{s}}$ represents the relative amplitude attenuation and $\delta_{\text {ellip }}=\left[E_{p}\right]-\left[E_{s}\right]$ is the phase lag of the $\mathrm{p}$ and $\mathrm{s}$ linearly polarized components upon reflection. We performed ellipsometer experiments using the Multiple-angle-of-incidence ellipsometry (MAIE) method which involves measurement of $\delta_{\text {ellip }}$ and $\psi_{\text {ellip }}$ values as a function of the angle of incidence (AOI), thus this information allows us to determine the dielectric function of the material studied. The dependence on the incidence angle of the ellipsometric angles was measured and averaged using a 4-zone nulling scheme. The analysis of the ellipsometric angles, $\delta_{\text {ellip }}$ and $\psi_{\text {ellip }}$, was done assuming that the surface can be described by the corresponding optical model. The accuracy of $\delta_{\text {ellip }}$ and $\psi_{\text {ellip }}$ is better than \pm 0.1 and \pm 0.05 degrees, respectively.

The ellipsometer is equipped with custom-built quartz tubes attached to 
both the source (laser) and the detector arm to enable measurements under liquid (in this case, decane) at variable angle of incidence (AOI). The intensity of the reflected light is minimized by a $90^{\circ}$ rotation of the analyser and the ellipsometric angles $\delta_{\text {ellip }}$ and $\psi_{\text {ellip }}$ are mapped. Drude model with standard Fresnel coefficients are used to relate the ellipticity $\rho=f\left(\delta_{\text {ellip }}, \psi_{\text {ellip }}\right)$ to determine the thickness of thin water layer. In case of mica, the bottom side of the substrate is roughened and coated with an index matched epoxy resin to suppress interference.

\subsubsection{Streaming Potential Measurements}

In order to determine the surface charge/potential of solid/water and oil/water interfaces, we performed streaming potential measurements using a ZetaCAD (CAD Instruments, France). The measurement cell consists of the two solid surface (material under investigation, $50 \mathrm{~mm} \times 30 \mathrm{~mm}$ ) at a separation of $100 \mu \mathrm{m}$. The liquid is forced to flow by a pressure drop P. When an aqueous solution of certain $\mathrm{pH}$ and ionic strength is driven through a channel bounded by the surfaces of interest, the streaming potential $\left(\mathrm{U}_{s t r}\right)$ generated by this flow was measured at different pressure difference values $(\Delta \mathrm{p})$. Streaming potentials are converted to $\zeta$-potentials using the standard Smoluchowski formula, $\zeta=\frac{\lambda \mu U_{s t r}}{\epsilon P}$. ( $\mu$ : viscosity; $\epsilon$ : permittivity of liquid; $\lambda$ : conductivity of the liquid). The pressure is ramped between -500 to +500 mbar with a step width of 10 mbar or less. While performing the experiment, it is assumed that the flow within the cell (i.e. between the mica substrates) is laminar irrespective of the differential pressure and the separation between the walls is large compared to the double layer thickness, which is fulfilled since Debye length $k_{D}^{-1} \sim 1-10 \mathrm{~nm}$ while the channel height is $\sim 100$ $\mu \mathrm{m}$.

\subsubsection{Atomic Force Microscopy}

An atomic force microscope (Bruker Multimode 8, USA) is used to investigate the adsorption of various species on the mineral surface. These experiments are performed ex-situ, with a silica tip on mica surface. The AFM is operated in non-contact mode (amplitude modulation) for obtaining atomic resolution at the mineral adsorption sites. The stiffness of the cantilever is measured $\left(\mathrm{k}_{\text {stiff }}\right)$, and with the information of the tip-geometry, the forcecurves are obtained. Reference measurements are done with silica tip-silica substrate interactions, and the interaction is substracted from the mica-silica 
tip interaction before fitting the result to a charge-regulation model for obtaining surface charge at mica-water interface.

\subsection{Results}

\subsubsection{Cation-valency Influenced Transition}

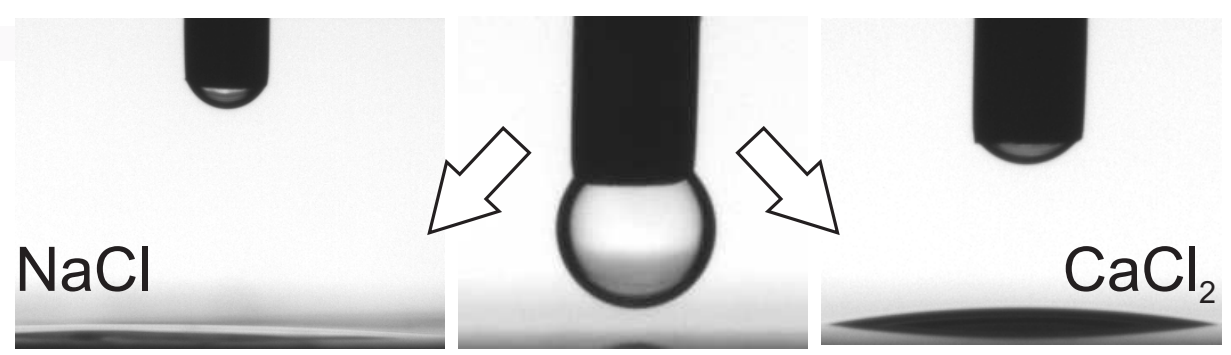

Figure 4.1: Wetting experiment on mica in ambient Decane with an aqueous drop of salt solution (middle); The difference between a monovalent and divalent salt solution is clear; a $1 \mathrm{M} \mathrm{NaCl}$ drop (at $\mathrm{pH} 7$ ) has $\sim 0^{\circ}$ contact angle on mica (left), almost completely wetting the substrate while a $1 \mathrm{M} \mathrm{CaCl}_{2}$ drop (at $\mathrm{pH} 7$ ) has a small finite contact angle on mica (right), indicating partial wetting.

Macroscopic contact angle (of aqueous drops) measurements are carried out on freshly cleaved mica or freshly piranha cleaned sillica surfaces in a decane environment. In the presence of representative monovalent salts, $\mathrm{NaCl}$ and $\mathrm{KCl}$ in the aqueous phase, we observe a wetting situation on mica as well as silica with imperceptible contact angles in ambient oil $\left(\theta<2^{\circ}\right.$, and thus a finite contact angle is not distinguishable within the resolution of the instrument). A silica surface immersed in oil is also completely wet by the aqueous phase containing divalent $\mathrm{CaCl}_{2}$ salt at all salt concentrations and $\mathrm{pH}$. The situation, however, changes significantly for wetting on mica. In Figure 4.1, we show the basic difference in $\mathrm{NaCl}$ and $\mathrm{CaCl}_{2}$ wetting: for a $1 \mathrm{M} \mathrm{NaCl}$ case, no perceivable drop is observed while for a $1 \mathrm{M} \mathrm{CaCl}_{2}$ case, the drop has a measurable contact angle. Above a thresold concentration (and at $\mathrm{pH}$ higher than 4 ), the aqueous phase with divalent cationic salt only partially wets the mica surface in ambient oil. The resulting contact angles, although small, are measureable using the optical goniometer.

Figure 4.2 shows that the salt concentration and the $\mathrm{pH}$ of the aqueous solution influences the wetting pattern, since with increasing concentration as 
well as increasing $\mathrm{pH}$, we observe an increase in contact angle.

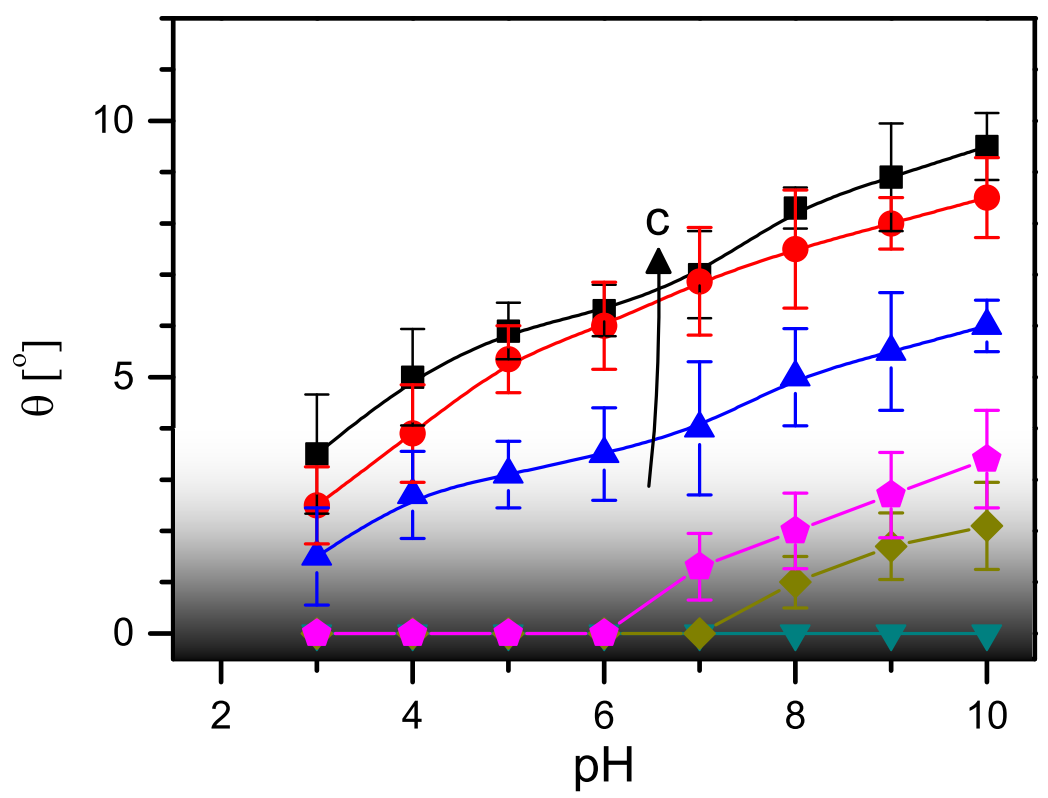

Figure 4.2: $\mathrm{CaCl}_{2}$ wetting on mica in ambient decane. Symbols: Equilibrium contact angle on mica vs $\mathrm{pH}$ for $\mathrm{CaCl}_{2}$ salt solutions of various concentrations; 1,10,30 mM (downward triangles), $50 \mathrm{mM}$ (olive diamonds), $80 \mathrm{mM}$ (purple pentagons), $100 \mathrm{mM}$ (blue triangles), $500 \mathrm{mM}$ (red circles), $1 \mathrm{M}$ (black squares). Solid lines: guides to the eye. Equilibrium contact angle datapoint is average of 25 independent measurements with the standard deviation shown. The shaded region indicates very low contact angles, which are close or below the sensitivity of the instrument. The arrow with the letter $\mathrm{c}$ denotes the direction of increasing salt concentration.

While macroscopically there is clearly a difference between monovalent and divalent salt solutions' wetting of mica in decane, it is interesting to investigate the microscopic origin of this difference. In the following section, we propose and discuss an ion-adsorption based argument for wetting alteration and the various consequences upon interactions in a wetting film. 


\subsection{Mechanistic Insight}

A change in contact angle can be attributed to a change in the corresponding interface energies (Fig.4.3a, left) i.e., the surface tensions of the various phases involved. Young's equation [15] is written as:

$$
\cos \theta=\frac{\gamma_{s o}-\gamma_{s w}}{\gamma_{o w}}
$$

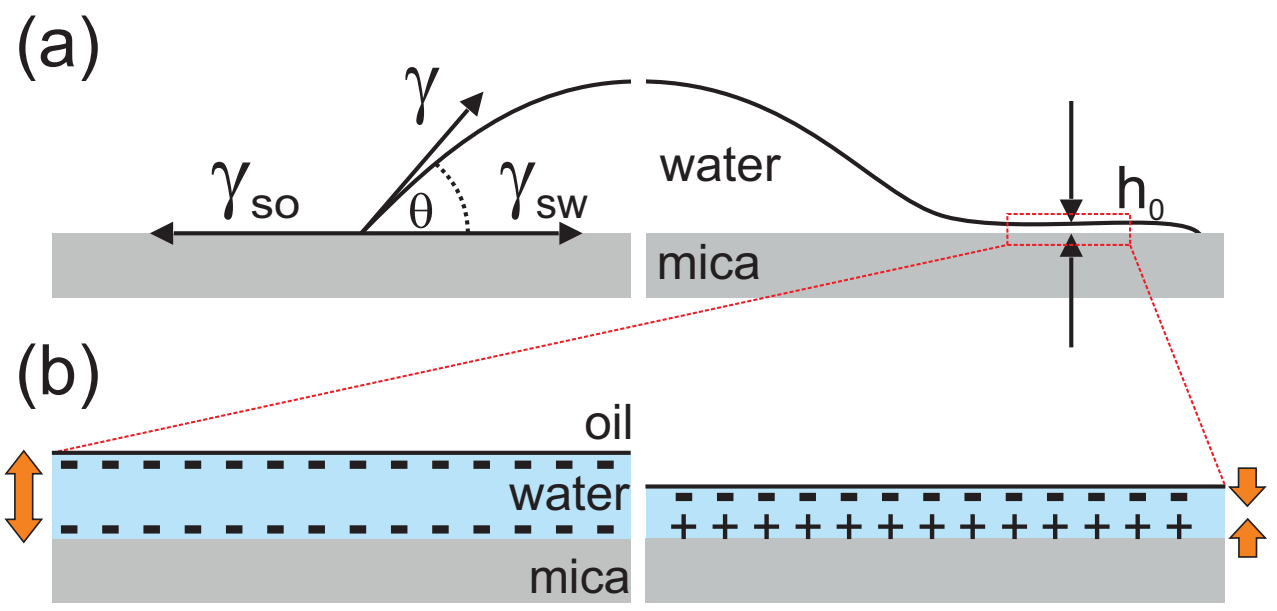

Figure 4.3: (a) Schematic of a partial wetting situation of water on mica in ambient decane; The force balance arising from interfacial tensions resulting in a finite contact angle $\theta$ (left), while such a finite contact angle implies a molecularly thin aqueous film co-existing with the drop (right) (b) Schematic of ionadsorption model; in the case of monovalent salt solutions (e.g. $\mathrm{NaCl}, \mathrm{KCl}$ ) both mica/water and oil/water interfaces are negatively charged, leading to a repulsive disjoining pressure (left); while in presence of divalent cations (e.g. $\mathrm{CaCl}_{2}$ ) charge reversal takes place at mica/water interface (right), leading to an attractive disjoining pressure (shown by the arrow).

where, $\theta$ is the contact angle and $\gamma_{s o}, \gamma_{s w}$ and $\gamma_{o w}$ are the interfacial tensions at mineral-oil, mineral-water and oil-water interfaces, respectively. It is well known, that if the salt concentration in the aqueous phase is increased, the depletion of cations leads to an increasing interfacial tension at a hydrophobic interface [17].

We have carried out pendant drop measurements of interface tension at oil/water interface and a small increase of the decane/water interfacial ten- 


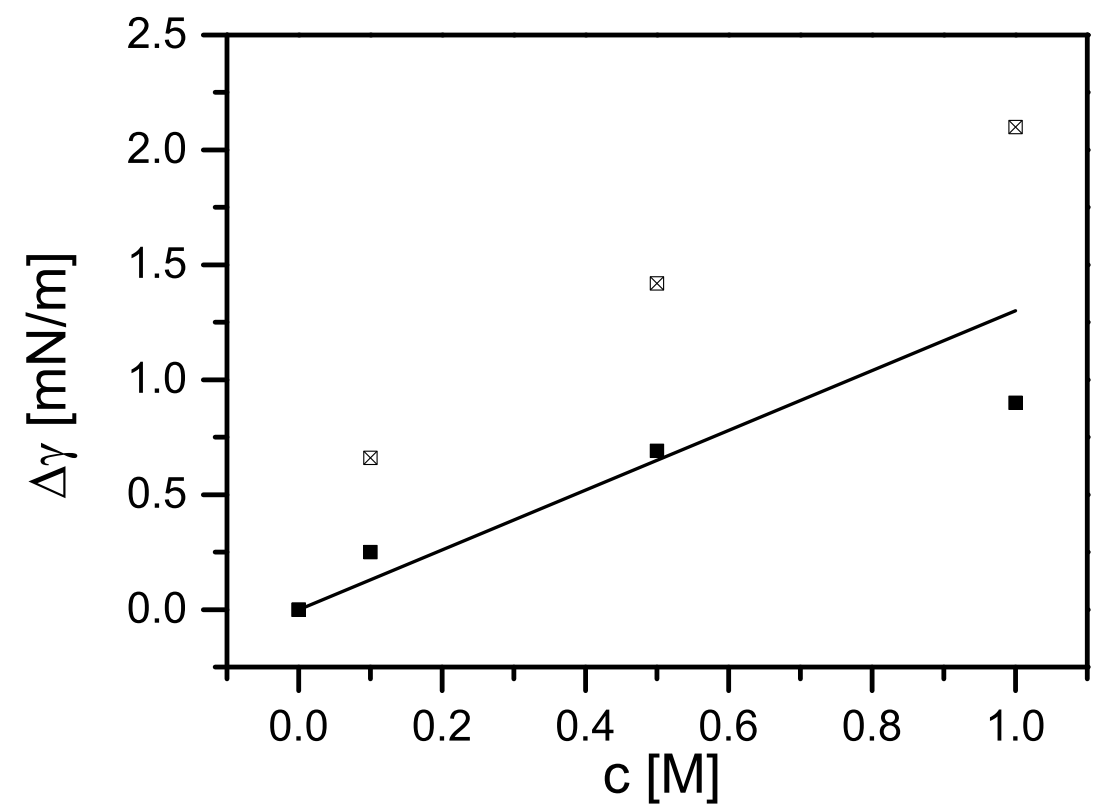

Figure 4.4: Interfacial tension (IFT) of decane/water interface with respect to salt concentration in the aqueous phase for $\mathrm{NaCl}$ (closed squares) and $\mathrm{CaCl}_{2}$ (crossed squares). The symbols are experimental datapoints at $\mathrm{pH}$ 6. Decane/$\mathrm{NaCl}$ IFT is compared with previous studies, denoted by the straight line [16].

sion is observed (Figure 4.4), which corroborate previous studies done in air or oil $[17,18]$. Based on the data, one might immediately argue, that an increasing $\gamma_{o w}$ results in an increasing $\theta$ from Equation 4.1. However, we have to reflect upon the microscopic picture of wetting as well, and take into consideration any other changes in interfacial tension. An increase in salt concentration normally results in a decrease of $\gamma_{s w}$ [19], since the formation of a double layer is more spontaneous with more ions present in the aqueous phase. A decreasing $\gamma_{s w}$, on the other hand, will result in a decrease in $\theta$, which is contrary to our experimental observations. Last but not least, the changes in $\gamma_{s o}$ will also influence the contact angle.

In chapter 2, we described the various wetting scenarios and their microscopic implications in detail. Wetting of a solid surface is effectively the formation of a thin layer of the wetting phase on the substrate, as shown in Figure $4.3 \mathrm{a}$, right. For such a thin aqueous film of equilibrium thickness $h_{0}$, 


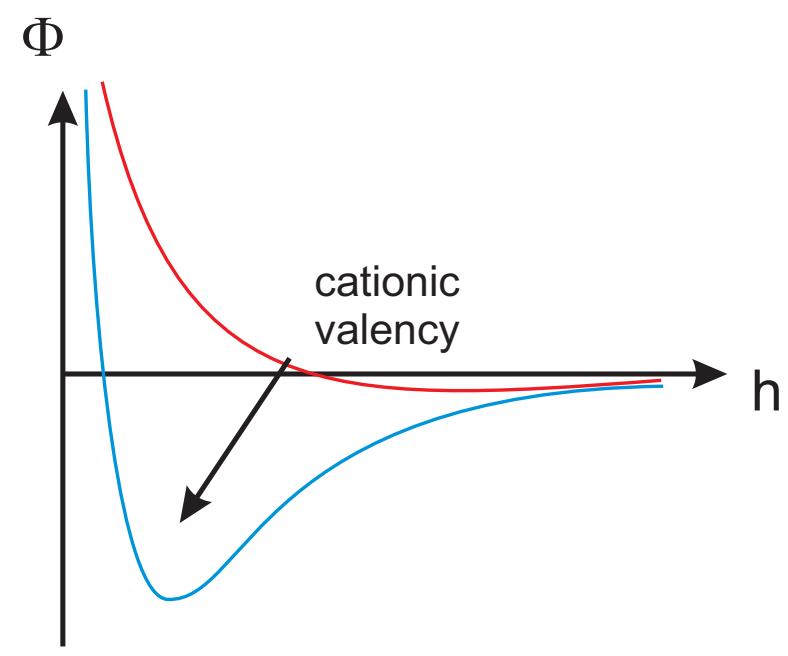

Figure 4.5: A schematic of gradual change in interface potential $(\Phi)$ in a micawater-oil system for our proposed mechanism: repulsive for a monovalent salt solution at lower concentration, which might have a shallow minimum (due to vdW interactions) at a relatively high concentration of the monovalent salt (red); attractive with a pronounced minimum for divalent salts at salt concentrations above a threshold value (blue).

$\gamma_{s o}$ can be written as:

$$
\gamma_{s o}=\gamma_{s w}+\gamma_{o w}+\Phi\left(h_{0}\right)
$$

where $\left.\Phi\left(h_{0}\right)\right)$ is the interaction potential in the thin aqueous film. Changes in $\Phi\left(h_{0}\right)$ are governed by the adsorption of counter-ions from this thin film to the interfaces. In the presence of a monovalent salt solution (schematic shown in Fig.4.3b, left), the counter-ion adsorption at the mineral interface is not strong enough to cause overcharging of the usually negatively charged mica-water interface. The oil-water interface remains negatively charged under all salt concentrations ( $>\mathrm{pH} 4$ ), since the mechanism of charging at a hydrophobic interface by autolysis of water molecules does not depend on cation adsorption $[20,21]$. As a result, there is a repulsive interaction between the mica-water and the oil-water interfaces or, in other words, the disjoining pressure in the thin aqueous film is positive, making the film inifinitely thick (compared to molecular length-scale, $\sim 10 \mathrm{~nm}$ film is considered infinitely thick) and creating a macroscopically indistinguishable contact angle. Divalent cations, on the other hand, have two units of charge per cation and when they adsorb to a mineral surface like mica, it causes 
overcharging above a threshold salt concentration, e.g., charge reversal has been reported for $\mathrm{CaCl}_{2}$ above $\sim 30 \mathrm{mM}$ salt concentration [22]. Fig.4.3b, right, depicts the schematic representation of the divalent situation. The oppositely charged mica-water and oil-water interfaces have an attractive interaction (or a negative disjoining pressure), creating a molecularly thin aqueous film along with a co-existing aqueous drop of macroscopically measurable contact angle. The situation is schematically portrayed in Figure 4.5. Hence the interaction potential $\Phi\left(h_{0}\right)$ is sensitive to the various microscopic interactions in the wetting film as well as macroscopic changes such as an increase or decrease in one of the interfacial tension values.

Our mechanistic picture of the experimental observations thus consists of two propositions: firstly, the existence of a molecularly thin wetting film of aqueous phase sandwiched between mineral and oil phases, and secondly, a charge reversal at mica surface in the presence of divalent salt solutions, which subsequently leads to an attractive interaction between the adsorbing interfaces. In the next section, we first focus on providing evidence for the first conjecture: namely, the thin wetting film associated with aqueous drops.

\subsubsection{Molecularly Thin Film}

To test the idea whether there is indeed a thin precursor layer of water spreading ahead of the contact line, we performed a series of experiments using imaging ellipsometry. Figure 4.6 shows the monochrome images of mica immersed in decane with a $1 \mathrm{M} \mathrm{NaCl}$ and a $1 \mathrm{M} \mathrm{CaCl}_{2}$ drop on it (all the measurements are performed 4 hours after depositing the drop thus allowing the drop sufficient time to spread). The fact that in both these cases we observe a clear three phase contact line, implies that in all our experiments under various salt concentration and $\mathrm{pH}$ conditions, we have a partial wetting situation. The wetting alteration observed in the experiments is a gradual divergence from a 'thick' to a 'thin' wetting film. We will discuss the role of van der Waals attraction as the origin of this partial wetting in greater detail in Chapter 5 and 6.

The thickness of the nm-thick film next to the wetting drops are calculated from the color map of ellipsometry experiments, as shown in Fig.4.7. For a bare mica substrate in decane, we obtain a rather homogeneous thickness of $(0.5 \pm 0.15) \mathrm{nm}$, averaged over an area of $1 \mathrm{~mm}^{2}$ in the centre of our field of view $\left(2 \times 2 \mathrm{~mm}^{2}\right.$, Fig.4.7a). The adsorption of water molecules on the 

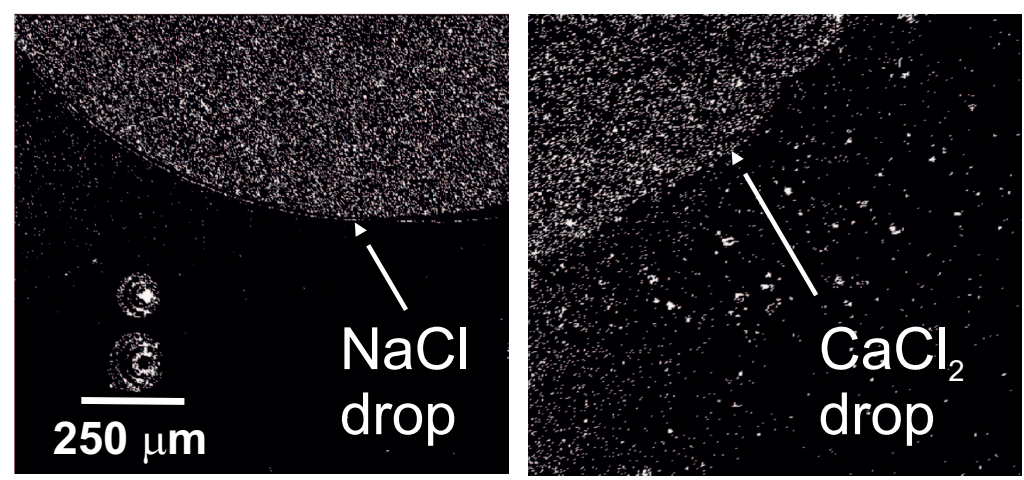

Figure 4.6: Monochrome ellipsometry images of $1 \mathrm{M} \mathrm{NaCl}$ and $1 \mathrm{M} \mathrm{CaCl}_{2}$ (both at $\mathrm{pH}$ 6) drops on mica in decane, depicting partial wetting situations in both cases.

mica surface creating such a hydration layer originates from the water saturating the decane $\left(5 \times 10^{-5} \mathrm{~g} / \mathrm{L}\right.$, at $\left.25^{\circ} \mathrm{C}\right)$.

For a $2 \mu \mathrm{l}$ drop of $\mathrm{CaCl}_{2}$ solution ( $\mathrm{pH} \sim 6,1 \mathrm{M}$ conc.) on mica in decane, the drop has a finite macroscopic contact angle of approximately $5^{o}\left( \pm 1^{o}\right.$ ). The 3-phase contact line for this drop is clearly visible, as shown in the upper left corner of Fig.4.7b. The corresponding aqeous film has a thickness of $(0.6 \pm 0.25) \mathrm{nm}$, which is comparable to the thickness of the aqueous film found on bare mica immersed in decane. The situation changes completely, however, when we deposit a drop of $\mathrm{NaCl}$ ( $\mathrm{pH} \sim 6,1 \mathrm{M}$ conc.) solution. In this case, the tiniest amount of liquid spreads out macroscop-
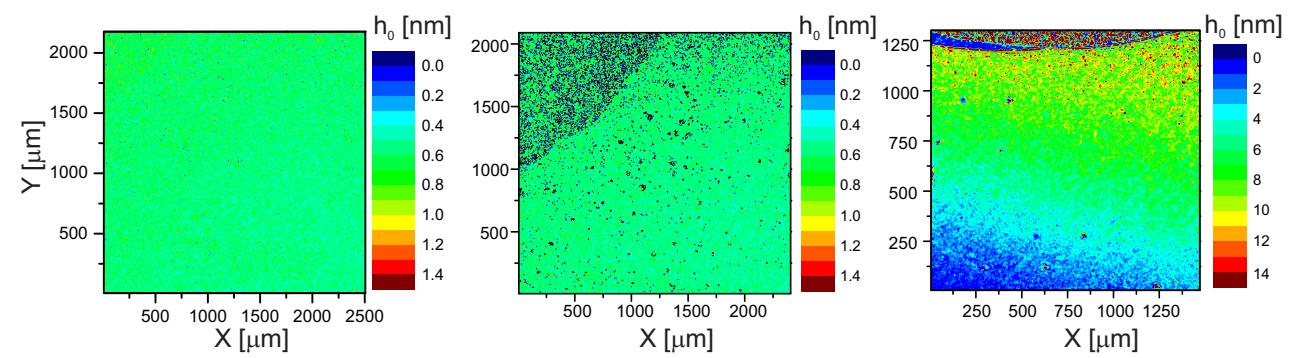

Figure 4.7: Thickness maps for (a) bare mica (b) aqueous drop of $1 \mathrm{M} \mathrm{CaCl}_{2}$ $(\mathrm{pH}=6)$ on mica and (c) aqueous drop of $1 \mathrm{M} \mathrm{NaCl}(\mathrm{pH}=6)$ on mica; all experiments in ambient decane. The outlines of the drops are visible for $\mathrm{CaCl}_{2}$ and $\mathrm{NaCl}$ cases. 
ically very quickly, as expected from our goniometry experiments and the proposed mechanism. Still, the spreading eventually slows down and it is possible to detect the edge of the now very large drop, which is very weakly curved at the top of Figure 4.7c. We see that there is a precursor layer with a thickness of approximately $10 \mathrm{~nm}$ close to the drop. Upon moving away from the drop, this thickness decreases to values of less than $1 \mathrm{~nm}$ over a macroscopic distance of approximately $1 \mathrm{~mm}$. This gradient suggests that the drop has not yet reached its final equilibrium shape since at equilibrium we expect a wetting film of constant thickness. (We could not reach this situation in our experiments since the spreading process is very slow).

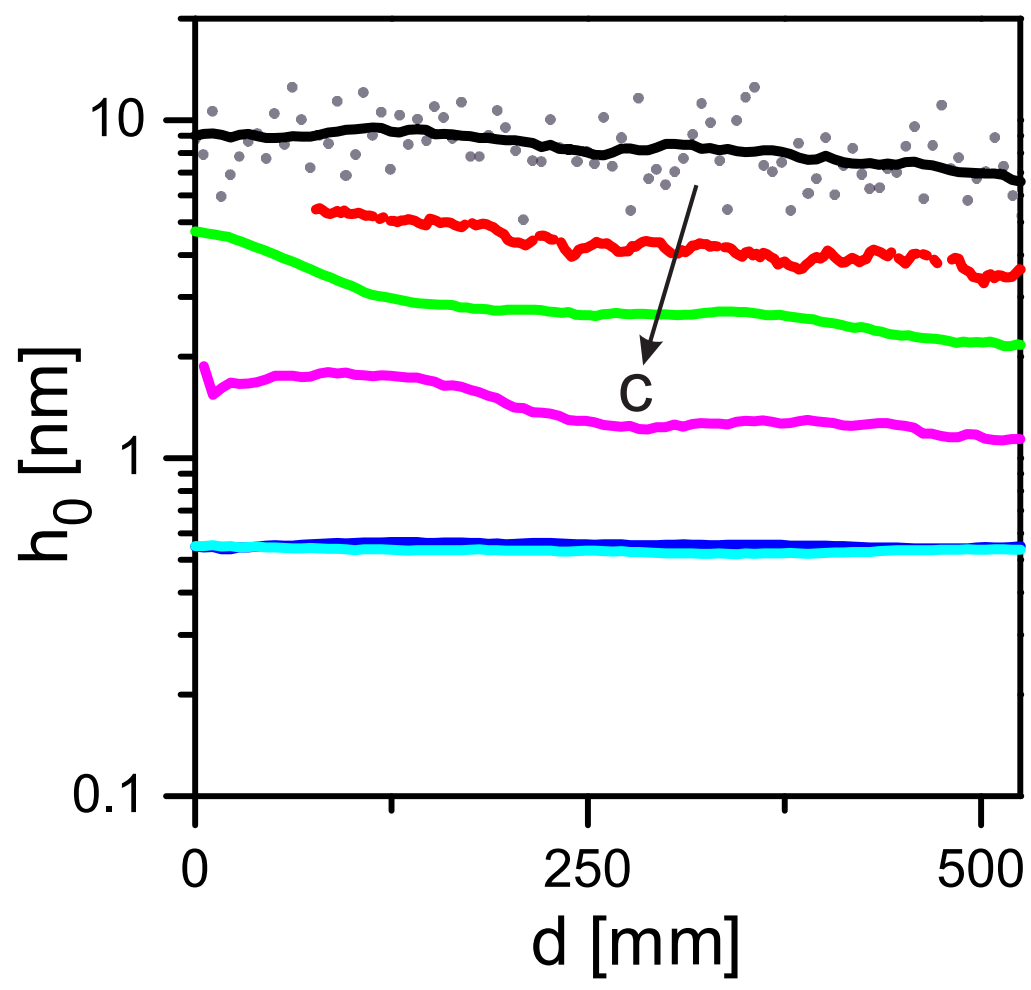

Figure 4.8: Equilibrium thickness of the aqueous film on mica in ambient decane $\left(\mathrm{h}_{0}\right)$ versus the distance away from the contact line (of the drop) for bare mica (dark blue); various concentrations of $\mathrm{CaCl}_{2}$ in the aqueous phase: $1 \mathrm{M}$ (light blue), $500 \mathrm{mM}$ (pink), $250 \mathrm{mM}$ (light green) and $100 \mathrm{mM}$ (red); $1 \mathrm{M} \mathrm{NaCl}$ (black) with the scatter for this last case shown (grey datapoints). All the aqueous drops are at a $\mathrm{pH}$ of 6 . The arrow and the letter ' $\mathrm{c}$ ' denote the direction of increasing $\mathrm{CaCl}_{2}$ concentration. 
Figure 4.8 summarizes the results of the average thickness of the water film as a function of distance from the edge of the drop $\left(\mathrm{CaCl}_{2}, \mathrm{NaCl}\right)$ and position (bare substrate), respectively. In this plot, we also present additional data at various $\mathrm{CaCl}_{2}$ concentrations to explore the evolution of the thickness upon approaching the wetting transition. The data confirm the proposed behavior of the wetting film in the vicinity of the wetting transition, which is in agreement with our proposition of the wetting mechanism. As the ionic strength (molar concentration) of $\mathrm{CaCl}_{2}$ decreases, the drops of its aqueous solution spread more on the solid surface, and we observe an increasing water layer thickness. Decreasing attraction (or increasing repulsion in the thin film) between the interfaces explains this thickness behaviour, whereas the strength of attraction depends on the available number of cations to bind to the mica surface (adsorption) i.e., the salt concentration in the aqueous phase.

Thus, the wetting alteration is actually a 'thick to thin' transition [15, 23] depending on certain conditions or interactions in the thin aqueous film. In the Mechanism section, we proposed that the surface charge reversal at mineral-aqueous interface would cause an attractive interaction in the precursor film, leading to a partial wetting regime. In the following section, we present experimental investigation of surface charge mica-water and oilwater interfaces.

\subsubsection{Surface Charge Measurement}

To test the hypothesis of charge reversal, we performed extensive $\zeta$-potential measurements for the specific conditions of our contact angle experiments. The $\zeta$-potential of the interface between mica and $\mathrm{NaCl}$ solutions is found to depend only moderately on $\mathrm{pH}$ and rather weakly on the salt concentration (Fig.4.9a). Increasing salt concentration by a factor of thousands increases the negative $\zeta$-potential by merely $20-50 \%$, depending on $\mathrm{pH}$. For $\mathrm{CaCl}_{2}$ solutions in mica, in contrast, the concentration dependence is much stronger (Fig.4.9b). While the $\mathrm{pH}$-dependence is equally weak, the $\zeta$-potential decreases from values around -40 to $-50 \mathrm{mV}$ down to values of $<-10 \mathrm{mV}$, even approaching zero at the lowest $\mathrm{pH}$ upon increasing the salt concentration from $1 \mathrm{mM}$ to $30 \mathrm{mM}$. In fact, according to [22], the mica-water interface charge $\sigma_{s w}$ is expected to reverse charge close to the highest concentrations investigated here. Atomic resolution AFM investigation (Figure 4.10) also captures stronger adsorption with $\mathrm{CaCl}_{2}$ in comparison to $\mathrm{NaCl}$. However, 

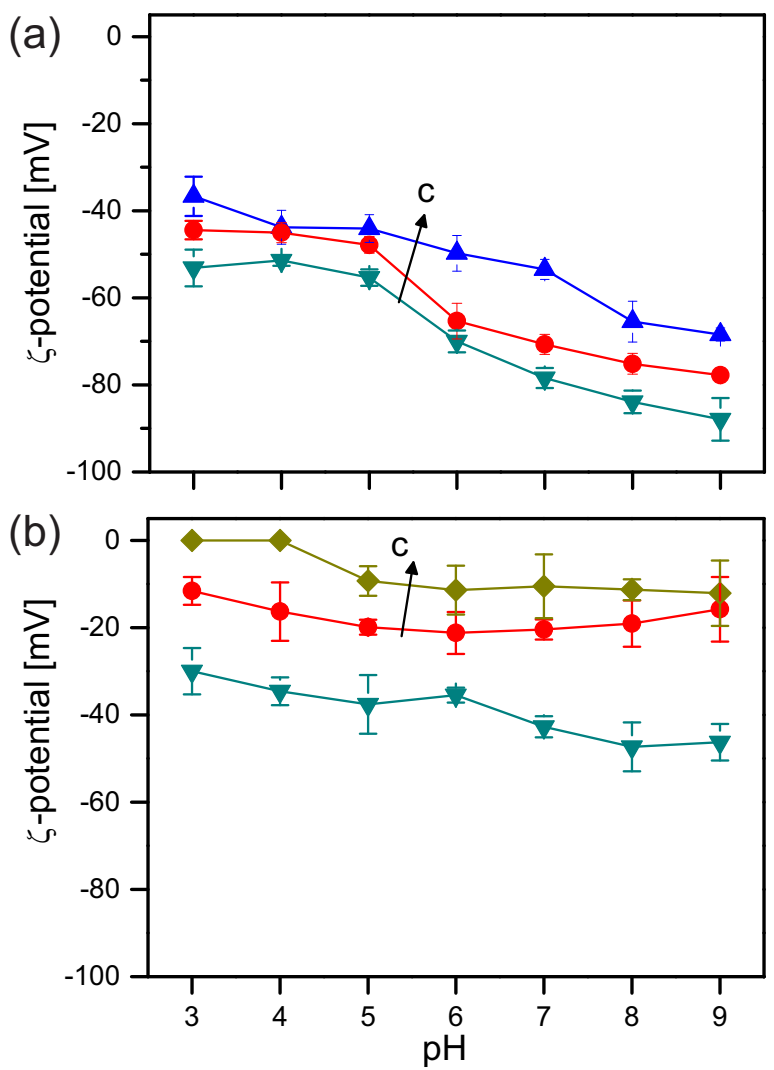

Figure 4.9: $\zeta$-potential at mica-water interface vs $\mathrm{pH}$ of aqueous (a) $\mathrm{NaCl}$ and (b) $\mathrm{CaCl}_{2}$ solution at various salt concentrations: $1 \mathrm{mM}$ (dark cyan downward triangles), $10 \mathrm{mM}$ (red circles), $30 \mathrm{mM}$ (olive diamonds) and $100 \mathrm{mM}$ (blue triangles). The symbols are average of 5 independent experiments under identical conditions with the standard error shown. The straight lines are guide to the eye. The arrows with the letters ' $c$ ' denote the direction of increasing salt concentration.

we could not observe any reversal of the sign of the $\zeta$-potential in our experiments, even at higher concentrations of $\mathrm{CaCl}_{2}$.

Measuring the surface charge of liquid oil-water interfaces is more challenging than for solid-liquid interfaces. In fact, it is not possible to measure this using a conventional streaming potential apparatus. However, interfacial charges of polymer-water interfaces for weakly polarizable polymers are usually found to depend rather weakly on the specific polymer. Hence, 
(a)

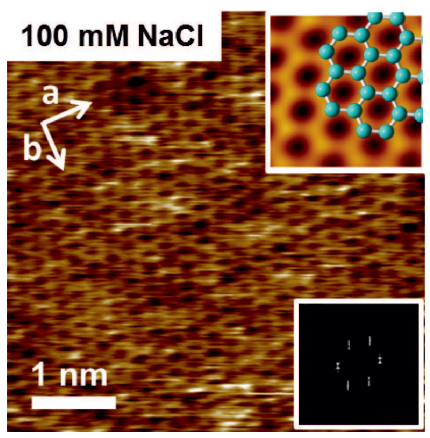

(b)

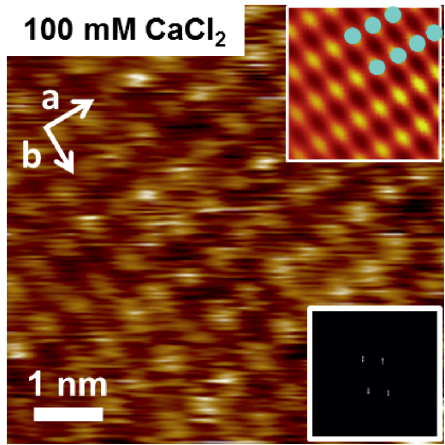

Figure 4.10: AFM images of mica-water interface showing the characteristic hexagonal lattice of mica in $100 \mathrm{mM} \mathrm{NaCl}$ solution (a), and a rectangular symmetry caused by (presumably hydrated) adsorbed $\mathrm{Ca}^{2+}$ ions in $100 \mathrm{mM} \mathrm{CaCl}_{2}$ (b). Insets: filtered zoomed views with overlaid lattice structure (top) and Fast Fourier Transform image of the same data (bottom). Measurements by I. Siretanu.

we decided to replace the liquid alkanes in our wetting experiments by a chemically identical alkane with a somewhat higher molecular weight that is solid at room temperature. We therefore coated a silicon wafer with a thin film of eicosane $\left(\mathrm{C}_{20} \mathrm{H}_{42}\right)$ using spin coating and used this sample in contact with water as a substitute for decane. The corresponding values of the $\zeta$-potential are shown in Figure 4.11. Indeed the $\zeta$-potential at this interface is negative, throughout the entire range of $\mathrm{pH}$ and concentrations of both $\mathrm{NaCl}$ and $\mathrm{CaCl}_{2}$ salts investigated, including up to $100 \mathrm{mM} \mathrm{CaCl}_{2}$.

Thus, the streaming potential measurements indicate that the oil-water interface is negatively charged irrespective of a monovalent or a divalent cationic salt in the aqueous phase but are not conclusive regarding the chargereversal at mica-water interface in the presence of divalent cationic salts. We note, however, that streaming potential measurements become increasingly unreliable for increasing salt concentrations. We presume that this is caused by the fundamental limitation of the electrokinetic approach and the applicability of the Smoluchowski equation that is used here (and in all common devices) to extract the $\zeta$-potential from streaming potential measurements. As the salt concentration increases, the Debye layer at the solid-aqueous interface is increasingly compressed. This leads to a reduced correlation between the $\zeta$-potential and the streaming potential. 


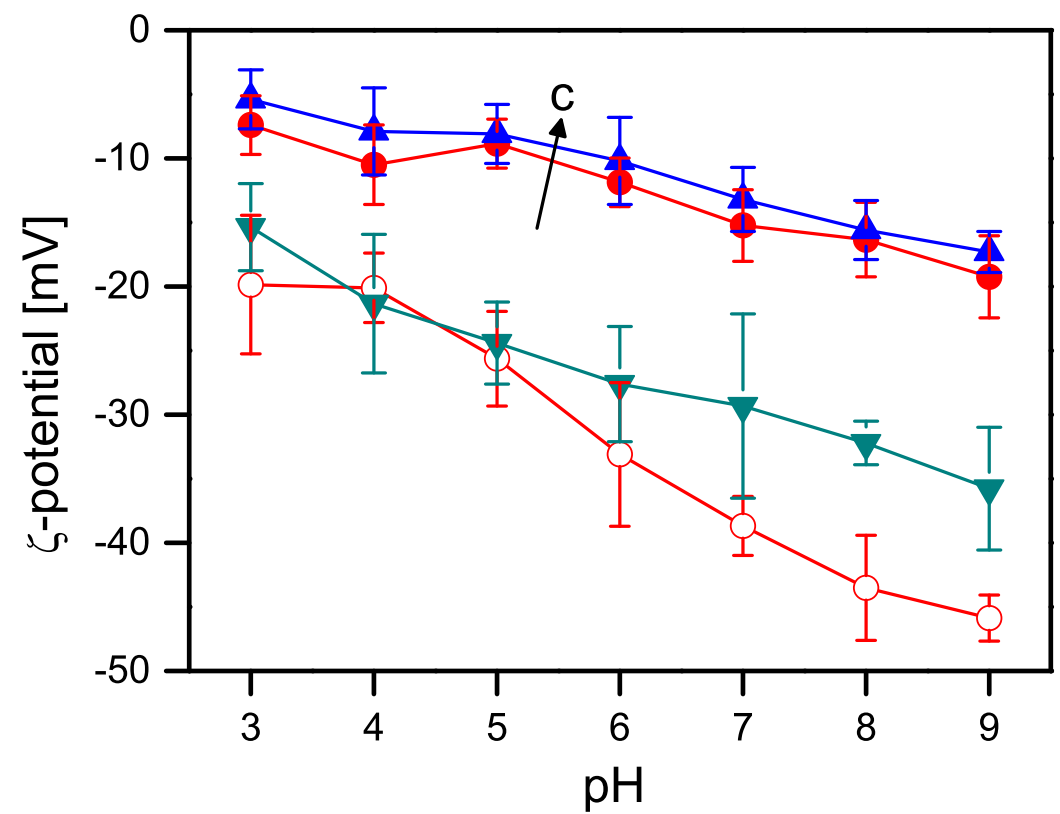

Figure 4.11: $\zeta$-potential at eicosane-water interface vs $\mathrm{pH}$ of aqueous $\mathrm{NaCl}$ (open symbols) and $\mathrm{CaCl}_{2}$ (closed symbols) solution at various salt concentrations: $1 \mathrm{mM}$ (dark cyan downward triangles), $10 \mathrm{mM}$ (red circles), $30 \mathrm{mM}$ (olive diamonds) and $100 \mathrm{mM}$ (blue triangles). The symbols are the average of 5 independent experiments under identical conditions with the standard error shown. The straight lines are to guide the eye. The arrow with the letter ' $c$ ' denotes the direction of increasing salt concentration.

Force spectroscopy measurements are more reliable in this context of surface charge measurement and AFM experiments have captured the chargereversal at mica-water interface as we switch from a monovalent cation to a divalent cation in the salt solution (Fig.4.12, [24]). When the monovalent cation in the aqueous phase is replaced by a divalent cation, the adsorption at mica surface is more pronounced. For $\mathrm{Ca}^{2+}$, above a certain concentration $\sim 30 \mathrm{mM}$ and $\mathrm{pH}$, the surface charge on mica clearly changes its sign. We conclude that the charge of our oil-water interfaces remains negative under all conditions of our wetting experiments, whereas the mica-water interface switches from negative to positive surface charge at sufficiently high $\mathrm{Ca}^{2+}$ concentrations and $\mathrm{pH}$, in agreement with the observed wetting transition. 


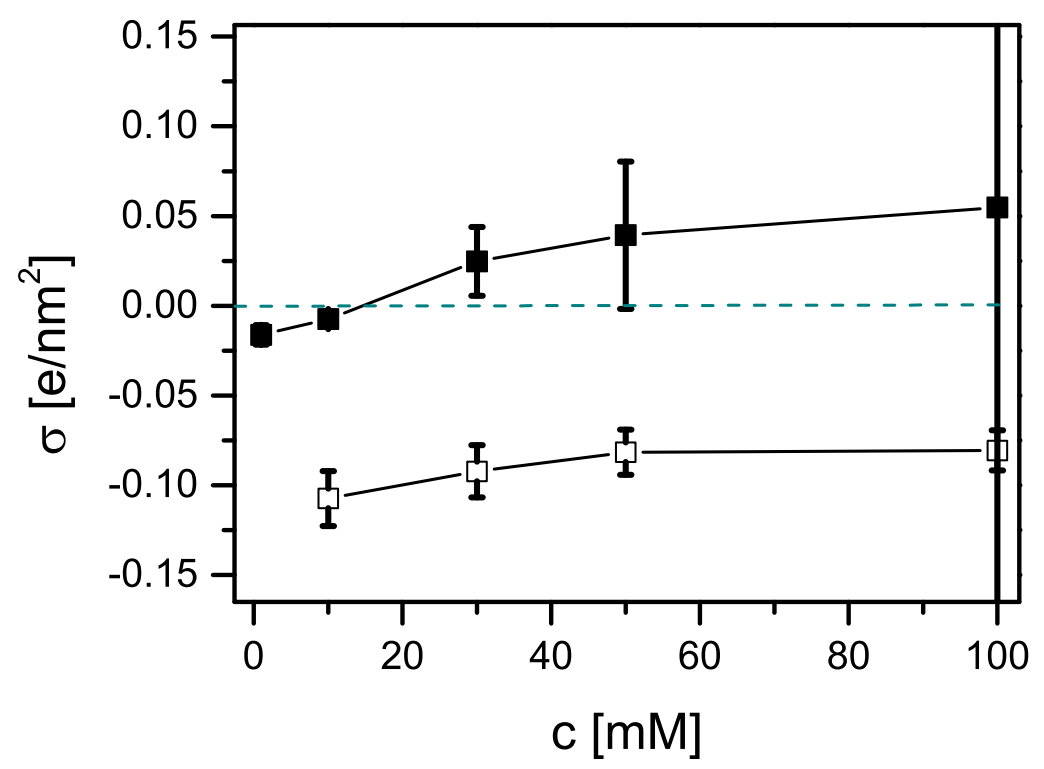

Figure 4.12: Surface charge $(\sigma)$ at the interface between mica and an aqueous solution of: $\mathrm{NaCl}$ (open symbols) and $\mathrm{CaCl}_{2}$ (closed symbols). The symbols are experimental datapoints based on 3 independent AFM experiments under identical conditions. The straight lines are to guide the eye. Figure reproduced with permission from [24].

\subsection{Concluding Remarks}

We have investigated and demonstrated wetting alteration of aqueous salt solution on two different mineral surfaces in ambient decane. We observe that a transition from near-zero contact angle to a finite contact angle (up to $\sim 10^{\circ}$ ) occurs on mica as we switch from a monovalent cation $\left(\mathrm{Na}^{+}\right.$or $\left.\mathrm{K}^{+}\right)$to a divalent cation $\left(\mathrm{Ca}^{2+}\right)$. Though the influence of a divalent species on charge reversal of a negatively charged mineral surface has been investigated before, in this chapter we have reported for the first time evidence of a transition in macroscopic contact angles for a mica-oil-water system influenced by the charge reversal at mica-water interface.

This transition from near-zero to a finite contact angle takes place above a threshold concentration of the divalent cation. For $\mathrm{Ca}^{2+}$, finite contact 
angles are measurable for concentrations $>50 \mathrm{mM}$. We have conclusively shown that the wetting phase exists as a thin film, and have pointed out that the macroscopic wetting pattern in our experiments and especially the wetting alteration is intricately related to the thickness of this film. The surface charge at the mica-water interface as well as oil-water interface plays a role in determining the electrostatic contribution in this film, as is evident from our streaming potential as well as AFM investigation.

We have mentioned influence of the electrostatic contribution in the thin film at various sections of this chapter, and also referred to the nature of interaction between two interfaces based on such contributions. However, we have not yet elaborated this point or investigated the influence of the interaction (between two adsorbing interfaces) upon the various wetting states of the aqueous phase. In the following chapter, we will formulate the theoretical framework in order to address the interaction potential between our interfaces, and especially the role of electrostatics in such interactions. 


\section{Bibliography}

[1] J. Yuan, X. Liu, O. Akbulut, J. Hu, S. L. Suib, J. Kong, and F. Stellacci. Superwetting nanowire membranes for selective absorption. Nat Nano, 3(6):332-336, 2008.

[2] S. Berg, H. Ott, S. A. Klapp, A. Schwing, R. Neiteler, N. Brussee, A. Makurat, L. Leu, F. Enzmann, J. Schwarz, M. Kersten, S. Irvine, and M. Stampanoni. Real-time 3d imaging of haines jumps in porous media flow. Proceedings of the National Academy of Sciences of the United States of America, 110(10):3755-3759, 2013.

[3] J. Murison, B. Semin, J. Baret, S. Herminghaus, M. Schroeter, and M. Brinkmann. Wetting heterogeneities in porous media control flow dissipation. Physical Review Applied, 2(3).

[4] X. Tian, H. Jin, J. Sainio, R. H. A. Ras, and O. Ikkala. Droplet and fluid gating by biomimetic janus membranes. Advanced Functional Materials, 24(38):6023-6028, 2014.

[5] T. Wong, S. Hoon Kang, Sindy K. Y. Tang, Elizabeth J. Smythe, Benjamin D. Hatton, A. Grinthal, and J. Aizenberg. Bioinspired selfrepairing slippery surfaces with pressure-stable omniphobicity. Nature, 477(7365):443-447, 2011.

[6] A. R. Kovscek, H. Wong, and C. J. Radke. A pore-level scenario for the development of mixed wettability in oil-reservoirs. Aiche Journal, 39(6):1072-1085, 1993.

[7] X. Zhao, M. J. Blunt, and J. Yao. Pore-scale modeling: Effects of wettability on waterflood oil recovery. Journal of Petroleum Science and Engineering, 71(3-4):169-178, 2010.

[8] L. W. Lake. Enhanced Oil Recovery. Prentice Hall, 1989. 
[9] A. Muggeridge, A. Cockin, K. Webb, H. Frampton, I. Collins, T. Moulds, and P. Salino. Recovery rates, enhanced oil recovery and technological limits. Philosophical Transactions of the Royal Society aMathematical Physical and Engineering Sciences, 372(2006), 2014.

[10] G. Q. Tang and N. R. Morrow. Influence of brine composition and fines migration on crude oil/brine/rock interactions and oil recovery. Journal of Petroleum Science and Engineering, 24(2-4):99-111, 1999.

[11] H. Aksulu, D. Hamso, S. Strand, T. Puntervold, and T. Austad. Evaluation of low-salinity enhanced oil recovery effects in sandstone: Effects of the temperature and ph gradient. Energy \& Fuels, 26(6):3497-3503, 2012.

[12] J. J. Sheng. Critical review of low-salinity waterflooding. Journal of Petroleum Science and Engineering, 120:216-224, 2014.

[13] J. Matthiesen, N. Bovet, E. Hilner, M. P. Andersson, D. A. Schmidt, K. J. Webb, K. N. Dalby, T. Hassenkam, J. Crouch, I. R. Collins, and S. L. S. Stipp. How naturally adsorbed material on minerals affects low salinity enhanced oil recovery. Energy \& Fuels, 28(8):4849-4858, 2014.

[14] A. Lager, K. J. Webb, C. J. J. Black, M. Singleton, and K. S. Sorbie. Low salinity oil recovery - an experimental investigation1. Petrophysics, 49:28-35, 2006.

[15] P.G. de Gennes. Wetting: statics and dynamics. Rev. Mod. Phys., 57(3):827-863, 1985.

[16] R. Aveyard and S. M. Saleem. Interfacial tensions at alkane-aqueous electrolyte interfaces. Journal of the Chemical Society, Faraday Transactions 1: Physical Chemistry in Condensed Phases, 72(0):1609-1617, 1976.

[17] P. K. Weissenborn and R. J. Pugh. Surface tension of aqueous solutions of electrolytes: Relationship with ion hydration, oxygen solubility, and bubble coalescence. Journal of Colloid and Interface Science, 184(2):550$563,1996$.

[18] A. P. dos Santos, A. Diehl, and Y. Levin. Surface tensions, surface potentials, and the Hofmeister series of electrolyte solutions. Langmuir, 26(13):10778-10783, 2010. 
[19] R. M. Pashley and J. N. Israelachvili. DLVO and hydration forces between mica surfaces in $\mathrm{Mg}^{2+}, \mathrm{Ca}^{2+}, \mathrm{Sr}^{2+}$, and $\mathrm{Ba}^{2+}$ chloride solutions. Journal of Colloid and Interface Science, 97(2):446-455, 1984.

[20] J. K. Beattie. The intrinsic charge on hydrophobic microfluidic substrates. Lab on a Chip, 6(11):1409-1411, 2006.

[21] J. Luetzenkirchen, T. Preocanin, and N. Kallay. A macroscopic water structure based model for describing charging phenomena at inert hydrophobic surfaces in aqueous electrolyte solutions. Phy. Chem. Chem. Phys., 10:4946, 2008.

[22] P. Kekicheff, S. Marcelja, T.J. Senden, and V.E. Shubin. Charge reversal seen in electrical double layer interaction of surfaces immersed in 2:1 calcium electrolyte. Jour. Chem. Phys., 99:6098, 1993.

[23] D. Bonn, J. Eggers, J. Indekeu, J. Meunier, and E. Rolley. Wetting and spreading. Reviews of Modern Physics, 81(2):739-805, 2009.

[24] N. Kumar. Surface charge characterization of heterogeneous rock/clay materials. Thesis, 2016. 


\section{CHAPTER 5}

\section{Electrostatic Modelling of Ion Adsorption Induced Wetting Transition}

\section{Abstract}

In the previous chapter, we showed that electrostatic forces between a mineral substrate and the oil-water interface play a key role in determining the wetting behavior of the system. However, the surface charge of these interfaces is often unknown, as it depends on several competing adsorption and desorption processes, which are not completely understood even for a standard adsorbing interface. In this chapter, we provide a description of this phenomenon. First we numerically solve the Poisson Boltzmann equation with constant charge boundary conditions. Based on the calculated surface charge, we derive the interface potential in the wetting film and obtain quantitative agreement with the experimental contact angles at the expense of some fitting parameters. Subsequently, we apply a linearized charge regulation model, which is justified for several systems of scientific interest, and allows a unified description of different substrates. We obtain analytical expressions for the wetting thresholds from an oil-wet to a water-wet behavior, and the value of contact angles for the finite wettability configurations. This approach describes each interface with only two parameters, each of which has a clear physical meaning and can be related to the chemistry of each substrate.

Parts of this chapter have been published as:

1. F. Mugele, B. Bera, A. Cavalli, I. Siretanu, A. Maestro, M. H.G. Duits, M. A. Cohen-Stuart, Dirk van den Ende, I. Stocker \& I. Collins. Ion adsorption-induced wetting transition in oilwater-mineral systems. Nature Scientific Reports, 5, DOI: 10.1038/srep10519 (2015).

2. A. Cavalli, B. Bera, H.T.M. van den Ende \& F. Mugele. An analytic model for the electrowetting properties of oil-water-solid systems. Physical Review E, Under review (2016). 


\subsection{Introduction}

In Chapter 4, we reported a thick to thin wetting transition for a decanewater-mica system [1], which was induced by modifying the salt concentration in the water. The transition is triggered by the positive charging of the mica substrate upon absorption of divalent $\mathrm{Ca}^{2+}$ cations. This is not observed for monovalent $\mathrm{NaCl}$ salt solutions. We have established in the previous chapter that electrostatic interactions are particularly relevant in determining the wetting behavior of an oil-water-rock system. We introduced the concept of interaction potential $\Phi(h)$ between two interfaces in the context of our wettability alteration experiments. We discussed that the character (attractive or repulsive) and strength of these interactions vary significantly depending on the nature of the substrate, and are modified by the presence of different ionic species.

In Chapter 2, we have discussed various wetting configurations in detail, and described the interaction potentials associated with these configurations. In the present chapter, we are going to use a theoretical model in order to investigate the role of electrostatic interactions on $\Phi(h)$, the effect of assuming specific boundary conditions upon $\Phi(h)$ for a realistic system, and finally, the influence of $\Phi(h)$ on the contact angles. We are going to use a Poisson-Boltzmann (PB) equation [2] approach to describe the electrostatic part of our ion-adsorption model. We use a complexation approach to describe the surface chemistry in this model.

The calculation of this electrostatic interaction potential is dependent on the assumptions about the individual interfaces which provide the boundary conditions for the PB equation. Many investigations of interaction potential between two ion-adsorbing interfaces have been carried out through the last half a century, with constant charge (CC) boundary conditions [3], or with constant potential (CP) [4] boundary conditions, or even a mixture of these boundary conditions (CC-CP or $\mathrm{CP}-\mathrm{CC}$ etc.) or a modified $\mathrm{PB}$ equation [5]. The charge regulation (CR) boundary condition is usually considered the most plausible assumption [6, 7] for ion-adsorbing interfaces, since it is expected that the binding of ions will lead to a 'non-fixed' surface charge, which depends on the electrostatic potential between the two interfaces.

We are going to focus on some of these boundary conditions in order to comment on the implication of assuming a particular set of boundary conditions on a realistic system. We begin with a numerical solution of the PB equation approach using constant charge (CC) boundary conditions, where the goal is to fit the experimental contact angles with our model. Subsequently, we 
have used a linearized charge-regulation (CR) boundary condition [8] in order to keep the model purely analytical. We scan a wide parameter space, tuning the boundary conditions continuously in order to gain insight into the suitability of a specific boundary condition.

\subsection{Complexation Model}

We start with the adsorption picture in a wetting film, as described in Chapter 2. An aqueous wetting film of thickness $h$ intercedes between the solid and the oil phases (Figure 5.1), where adsorption of ions occurs at the individual interfaces. Both solid-water and oil-water interfaces can bind ions and acquire surface charge densities $\sigma_{1}$ and $\sigma_{2}$, respectively. We consider only the minerals which acquire a negative charge at the interface when in contact with a saline aqueous phase (e.g., mica, silica). Mica obtains a negative charge in an aqueous solution when the $\mathrm{K}^{+}$in the crystal structure is dissociated: $S K \rightleftharpoons S^{-}+K^{+}$, where $\mathrm{S}^{-}$denotes the sites at the interface. Silica, on the hand, becomes negatively charged in aqueous solution through the deprotonation reaction: $S H \rightleftharpoons S^{-}+H^{+}$. Now, we will take two adsorption reactions on the surface into account. First, we consider the protonation of mica/re-protonation of silica surface:

$$
S H \rightleftharpoons S^{-}+H^{+}
$$

with the equlibrium constant $\mathrm{K}_{H}$ given as:

$$
K_{H}=\frac{\left\{S^{-}\right\}\left[H^{+}\right]}{\{S H\}}
$$

where, $\{A\}$ is the surface density of species $\mathrm{A}$ (in sites $/ \mathrm{nm}^{2}$ ) and [B] is the volume density of species B (in mol/l). Now we assume that the $S^{-}$sites are occupied by a cation $\mathrm{C}$ with valency $\mathrm{Z}_{c}$ through adsorption:

$$
S^{-}+C^{\left(Z_{c}\right)+} \rightleftharpoons S C^{\left(Z_{c}-1\right)+}
$$

In this case the corresponding equilibrium constant is given by:

$$
K_{C}=\frac{\left\{S^{-}\right\}\left[C^{\left(Z_{c}\right)+}\right]}{\left\{S C^{\left(Z_{c}-1\right)+}\right\}}
$$




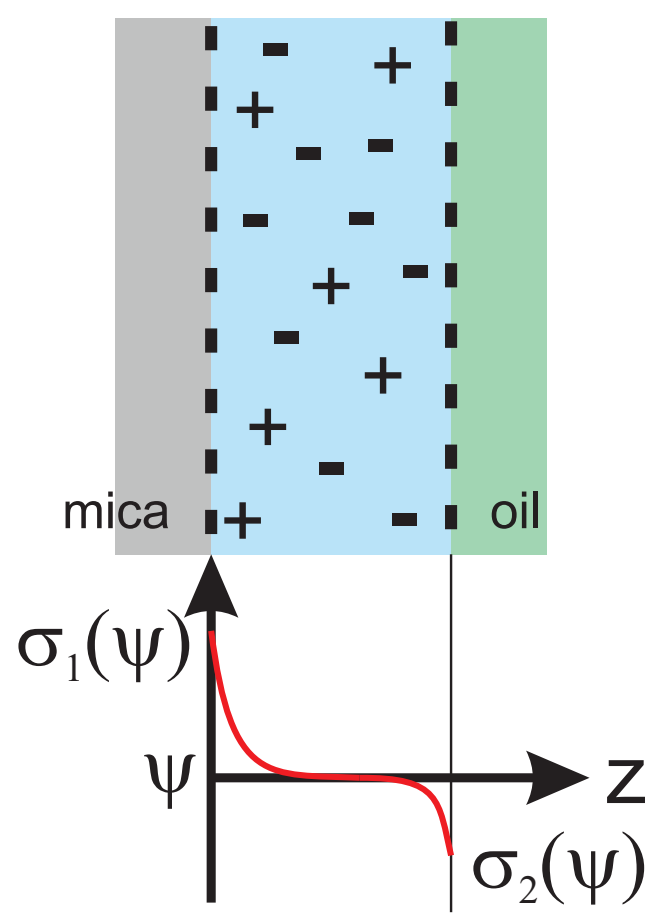

Figure 5.1: Schematic of an aqueous wetting film between two adsorbing platelike interfaces (mica and oil) along with the ion distribution.

Then, recalling that the total site density $(\Gamma)$ is constant, we can write the sum of all surface concentration contributions as:

$$
\left\{S^{-}\right\}+\{S H\}+\left\{S C^{\left(Z_{c}-1\right)+}\right\}=\Gamma
$$

From this system of equations, we can find the values of $\left\{S^{-}\right\},\{S H\}$ and $\left\{S C^{\left(Z_{c}-1\right)+}\right\}$, which, in turn, provide the surface charge $\sigma_{s}$ at the interface, originating from chemical reactions considered, and is given by the following expression:

$$
\sigma_{s}=\frac{e \Gamma\left(\frac{\left(Z_{c}-1\right)\left[C^{\left(Z_{c}\right)+}\right]}{K_{C}}-1\right)}{1+\frac{\left[H^{+}\right]}{K_{H}}+\frac{\left[C^{\left(Z_{c}\right)+}\right]}{K_{C}}}
$$

Now, we can relate the volume density of the cation $\left[C^{\left(Z_{c}\right)}\right]$ and of the proton $\left[\mathrm{H}^{+}\right]$at the interface with their respective bulk concentrations, using 
the Boltzmann distribution:

$$
\begin{gathered}
{\left[H^{+}\right]=\left[H^{+}\right]_{\infty} \exp \left(-\frac{e \phi_{s}}{k_{B} T}\right)} \\
{\left[C^{\left(Z_{c}\right)+}\right]=\left[C^{\left(Z_{c}\right)+}\right]_{\infty} \exp \left(-\frac{Z_{c} e \phi_{s}}{k_{B} T}\right)}
\end{gathered}
$$

Substituting Eq.5.5a and Eq.5.5b in Eq.5.4, we obtain the expression for $\sigma_{s}$ :

$$
\sigma_{s}\left(\phi_{s}\right)=\frac{e \Gamma\left(\frac{\left(Z_{c}-1\right)\left[C^{\left(Z_{c}\right)+}\right]_{\infty} \exp \left(-\frac{Z_{c e} \phi_{s}}{k_{B} T}\right)}{K_{C}}-1\right)}{1+\frac{\left[H^{+}\right]_{\infty} \exp \left(-\frac{e \phi_{s}}{k_{B} T}\right)}{K_{H}}+\frac{\left[C^{\left(Z_{c}\right)+}\right]_{\infty} \exp \left(-\frac{Z_{c e \phi_{s}}}{k_{B} T}\right)}{K_{C}}}
$$

Thus, when we know the total site density $(\Gamma)$ of the mineral phase, we can obtain a specific relation between the surface charge $\sigma_{s}$ for specific values of $K_{H}$ and $K_{C}$ and known bulk concentrations of cations.

For the oil-water interface, autolysis of water is considered to be the primary charge generation mechanism: $\mathrm{H}_{2} \mathrm{O} \rightleftharpoons \mathrm{H}^{+}+\mathrm{OH}^{-}$. We assume that both $\mathrm{H}^{+}$and $\mathrm{OH}^{-}$ions are generated at the interface, but the $\mathrm{OH}^{-}$ion diffuse away at a slower rate than the $\mathrm{H}^{+}$ions, causing the negative charge [9]. It is also assumed that the proton and cation adsorption at this interface is much weaker compared to the solid substrate, and is negligible. Thus, using the appropriate $\mathrm{pK}$ values of $\mathrm{OH}^{-}$and $\mathrm{H}^{+}$, we arrive at an expression of surface charge at the oil-water interface, which is similar to the expression in Equation 5.6:

$$
\sigma_{o w}\left(\phi_{o w}\right)=-\left\{O H^{-}\right\}=e \Gamma_{o w} \frac{-1}{1+\frac{\left[H^{+}\right]_{\infty} \exp \left(-\frac{e \phi_{s}}{k_{B} T}\right)}{K_{H, w}}}
$$

where, $\Gamma_{o w}$ is the site density for adsorption at oil-water interface, while $K_{H, w}$ is adsorption equilibrium constant for autolysis reaction.

In Chapter 4, we have described the experimental investigation of mica surface charge using the streaming potential method and atomic force microscopy. In Figure 5.2, these surface charge values (circles) were compared with the surface charge calculated from the complexation model (solid lines) 


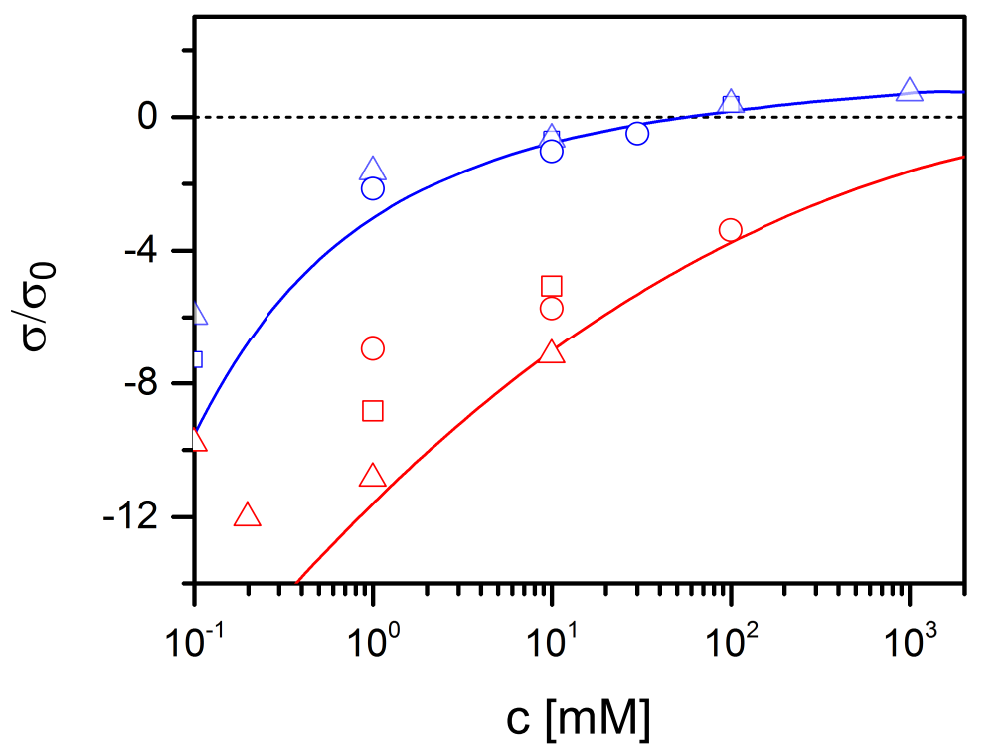

Figure 5.2: Mica surface charge (dimensionless) calculated from $\zeta$-potential measurements (circles) vs. concentration of solutions of $\mathrm{NaCl}$ (red) and $\mathrm{CaCl}_{2}$ (blue) at $\mathrm{pH}$ 6. Solid lines: surface complexation model predictions with $p K_{\mathrm{Na}^{+}}=4, p K_{\mathrm{Ca}^{2+}}=1.5$ and $p K_{H^{+}}=5.9$ [10]. Blue triangles: AFM data from [11]: blue squares [12], red triangles [10], red squares [13]: SFA measurements. The charge density is normalized by the characteristic scale $\sigma_{0}$ arising from the PB equation, $\sigma_{0}=\epsilon \epsilon_{0} k_{B} T \kappa_{D} / e$, where $\kappa_{D}$ is the Debye parameter.

described above using parameters from [10]. The red circles and the red line correspond to experiments and calculations for the mica- $\mathrm{NaCl}$ interface at $\mathrm{pH} 7$, while the blue circles and blue line correspond to mica-CaCl $\mathrm{C}_{2}$ interface at the same $\mathrm{pH}$. The agreement is corroborated with other surface force apparatus (SFA) and AFM studies as well [10, 12]. As expected and described in Chapter 4, for $\mathrm{NaCl}$ salt solution, a negative surface charge prevails on mica even at a concentration much higher than our experimental limit (1M). For $\mathrm{CaCl}_{2}$, on the other hand, the complexation model estimates a stronger adsorption, resulting in a charge-reversal at $\sim 50 \mathrm{mM}$ salt concentration.

The surface complexation model, however, estimates a negative surface charge at the oil-water interface irrespective of the cation valency or salt concentration. According to the complexation model, the oil-water interface is always negatively charged above $\mathrm{pH} 4$. The comparison of the experimental 


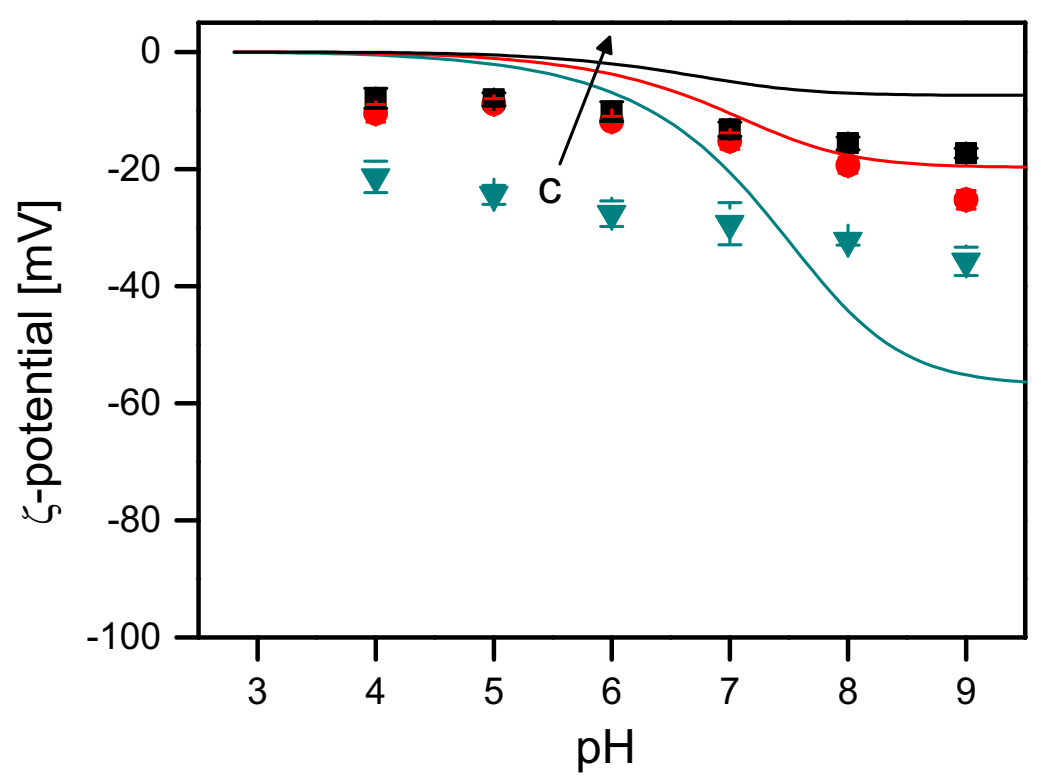

Figure 5.3: Decane-water $\zeta$-potential calculated from complexation models (solid lines) vs $\mathrm{pH}$ of aqueous phase containing $\mathrm{CaCl}_{2}$ at various concentrations: $1 \mathrm{mM}$ (dark cyan), $10 \mathrm{mM}$ (red) and $100 \mathrm{mM}$ (black). Adsorption constants for complexation model are: $p K_{\mathrm{Na}^{+}}=p K_{\mathrm{Ca}^{2+}}=-1, p K_{\mathrm{H}^{+}}=7$ [14]. The symbols denote streaming potential measurements for eicosane-water interface where the aqueous phase contains $\mathrm{CaCl}_{2}$ at the same concentrations.

$\zeta$-potential values obtained at eicosane-water interface (solid symbols, described in detail in chapter 4) and the decane-water interface $\zeta$-potential obtained from the complexation model (solid lines) is shown in Figure 5.3. The agreement between the experimental $\zeta$-potential values and the estimated values from the model is poor. One of the reasons is that the experimental system is eicosane $\left(\mathrm{C}_{20} \mathrm{H}_{42}\right)$, which has a higher number of carbon atoms in the chain and is a solid at room-temperature, while the complexation model estimations are for decane interface based on the wetting experiments. Another reason in the disagreement between the experimental and the calculated values might be the inadequacy of a simplistic cation depletion model for a hydrophobic interface. We have simulated the hydrophobic interface as an interface with cation depletion and subsequent $\mathrm{OH}^{-}$adsorption, thus leading to a negative charge at the interface. Recent studies [14] have shown that more complex adsorption reactions should be accounted for a realistic description of the oil-water interface. However, we will show in the follow- 
ing sections that the presence of a wetting transition is independent of the exact model implementation, especially at the oil-water interface.

\subsection{Constant Charge Model}

Now that we have found a relation between the surface charge $\sigma_{s}$ and the surface potential $\phi_{s}$, for both the mineral-water and the oil-water interfaces, we can derive an expression for the electrostatic potential $\phi$ in the film. We solve the PB equation between these two interfaces using these $\sigma_{s}$ or $\phi_{s}$ values as boundary conditions:

$$
\frac{\partial^{2} \phi}{\partial z^{2}}=-\frac{e}{\epsilon \epsilon_{0}} \sum_{i} Z_{i}\left[C_{i}\right]_{\infty} \exp \left(-\frac{Z_{i} e \phi}{k_{B} T}\right)
$$

We have used a constant charge (CC) boundary condition for solving Equation 5.8:

$$
\begin{aligned}
& \left.\frac{\partial \phi}{\partial z}\right|_{z=0}=-\sigma_{s 1, \infty} \\
& \left.\frac{\partial \phi}{\partial z}\right|_{z=h}=\sigma_{s 2, \infty}
\end{aligned}
$$

where, $\sigma_{s 1, \infty}$ and $\sigma_{s 2, \infty}$ are surface charges of interfaces 1 (solid-water) and 2 (oil-water), respectively. These values follow from Grahame's equation assuming that the two interfaces are far away from each other.

The reason for using a constant charge (CC) model is that the PB equation to be solved already requires numerical solution and charge regulation (CR) boundary condition adds complication to this numerical solution. We will observe later in the Discussion section, that this approximation is adequate for a certain range of $\mathrm{pH}$ and salt concentrations.

Once $\phi$ is calculated from this equation, the disjoining pressure $\Pi_{e l}$ is given by: $\Pi_{e l}(z)=\frac{\epsilon \epsilon_{0}}{2}\left[\kappa_{D}^{2} \phi^{2}-\left(\frac{\partial \phi}{\partial z}\right)^{2}\right]$, and subsequently, the electrostatic contribution of the interaction potential between the interfaces is given by: $\Phi_{e l}(h)=-\int_{\infty}^{h} \Pi_{e l}(x) d x$.

We also account for the contribution of van der Waal's forces $\Phi_{v d W}(h)$ and contribution of short-range chemical hydration forces $\Phi_{\text {hyd }}(h): \Phi(h)=$ 
$\Phi_{e l}(h)+\Phi_{v d W}(h)+\Phi_{h y d}(h)$. The van der Waal's forces contribution is expressed as: $\Phi_{v d W}(h)=\frac{A}{12 \pi h^{2}}$, where A is the Hamaker constant. Generally, A varies very weakly with $\mathrm{pH}$ and salt concentration, and they are not expected to change sign for our experiments. We use a Hamaker constant $\mathrm{A}=-0.4 \times 10^{-21}$, where the choice of $\mathrm{A}$ is restricted by the experimental constraint that contact angles for $\mathrm{NaCl}$ and $\mathrm{KCl}$, for all salt concentrations and $\mathrm{pH}$, should not exceed $2^{\circ}$. This negative Hamaker constant implies long range partial wetting, which arises from the fact that water has a lower refractive index than both mica and oil. $\Phi_{h y d}(h)$, the hydration contribution, is assumed as an exponentially decaying function and is given by: $\Phi_{h y d}(h)=\Phi_{h y d}^{0} \exp (-h / \lambda)$. The amplitude $\Phi_{h y d}^{0}$ of this function is of the order $10 \ldots . .50 \mathrm{~mJ} / \mathrm{m}^{2}$, and the decay length $\lambda<1 \mathrm{~nm}[11,15]$.
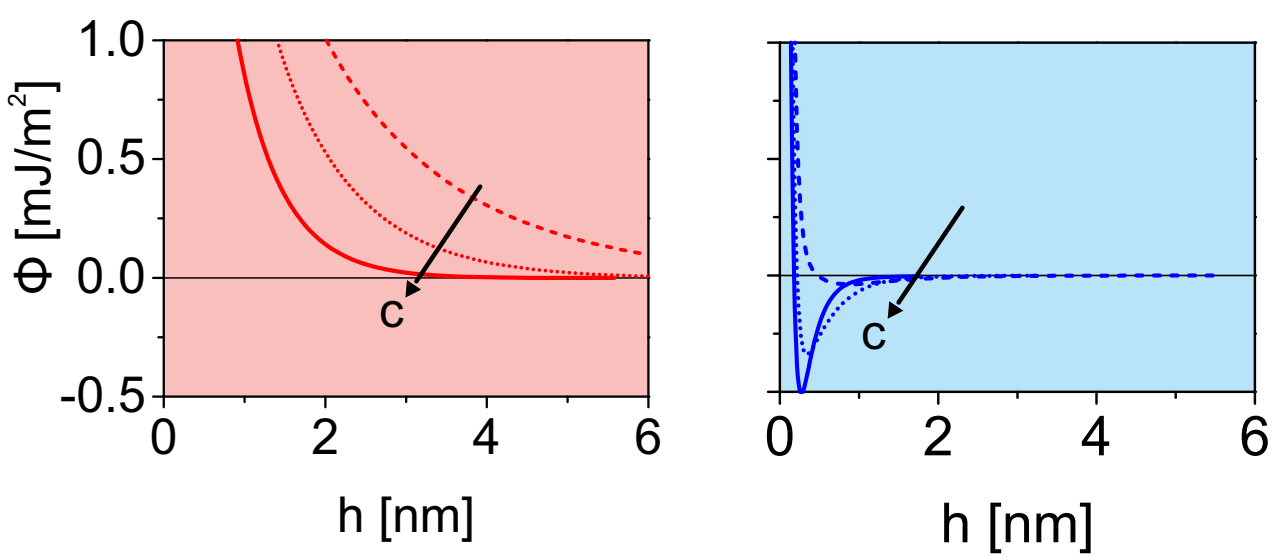

Figure 5.4: Effective interface potential for surface charges of equal (left, red lines, mica- $\mathrm{NaCl}$ solution at $\mathrm{pH}$ 6-oil) and of opposite sign (right, blue lines, mica- $\mathrm{CaCl}_{2}$ solution at $\mathrm{pH}$ 6-oil), leading to near-zero and finite contact angles, respectively. Lines denote salt concentrations: $1 \mathrm{mM}$ (dashed lines), 10mM (dotted lines) \& 100mM (solid lines). The arrows with the letter c denote the direction of increasing salt concentration.

Adding up all the contributions, we find that for sufficiently high divalent cation concentrations (for example, the case of $\mathrm{Ca}^{2+}$ shown in Figure 5.4 , right), $\Phi(h)$ indeed develops a pronounced minimum at small $\mathrm{h}_{0}$, corresponding to water contact angles up to $10^{\circ}$. For $\mathrm{Na}^{+}$and $\mathrm{K}^{+}$(Fig.5.4, left), however, a very shallow minimum corresponding to a small but finite contact angle appears, due of the dominance of attractive van der Waals interactions (i.e. $\mathrm{A}<0$ ) for large film values of $h$. 
We recall the relation $\gamma_{s o}=\gamma_{s w}+\gamma_{o w}+\Phi\left(h_{0}\right)$ from Chapter 4, where $\gamma_{s o}$, $\gamma_{s w}$ and $\gamma_{o w}$ are the interfacial tensions at mineral-oil, mineral-water and oilwater interfaces, respectively. Using Young's equation, this relation can be rewritten as:

$$
\cos \theta=1+\frac{\Phi\left(h_{0}\right)}{\gamma_{o w}}
$$

Here, $\Phi\left(h_{0}\right)$ is the total interaction potential between the mica-water and oil-water interfaces with an aqueous film of constant thickness $h_{0}$. Figure $5.5 \mathrm{a}$ shows the contact angles calculated for $\mathrm{CaCl}_{2}$ drops at various salt concentrations and $\mathrm{pH}$, which seems to have captured all the salient features of our experimental observations of $\theta$ for similar conditions (Fig.5.5b, which shows the experimental datapoints connected with smoothed interpolation lines), including in particular the transition from near zero contact angles at low divalent ion concentration and $\mathrm{pH}$ to values of $\theta \sim 10^{\circ}$ at high $\mathrm{Ca}^{2+}$ concentration and $\mathrm{pH}$. For monovalent cations on mica and for all salts on silica, the same calculation invariably results in repulsive electrostatic forces and hence negligibly small contact angles $\left(<2^{\circ}\right)$.

\subsection{Linearized Charge Regulation Model}

The model described so far requires numerical solution in order to calculate the electrostatic potential from the PB equation using constant charge (CC) boundary conditions, and for the subsequent calculation of disjoining pressure and interaction potential. In this section, we analyze the same system using a linearized $\mathrm{PB}$ equation, with a linearized charge regulation (CR) boundary condition. This approach will allow us to build a purely analytical model. In this section, we do not consider the van der Waal's or hydration contributions of the interaction potential, and we focus on the electrostatic effect in our solid-water-oil system.

The linearized PB (or Debye-Huckel) equation with the charge regulation $(\mathrm{CR})$ boundary condition is given by: 

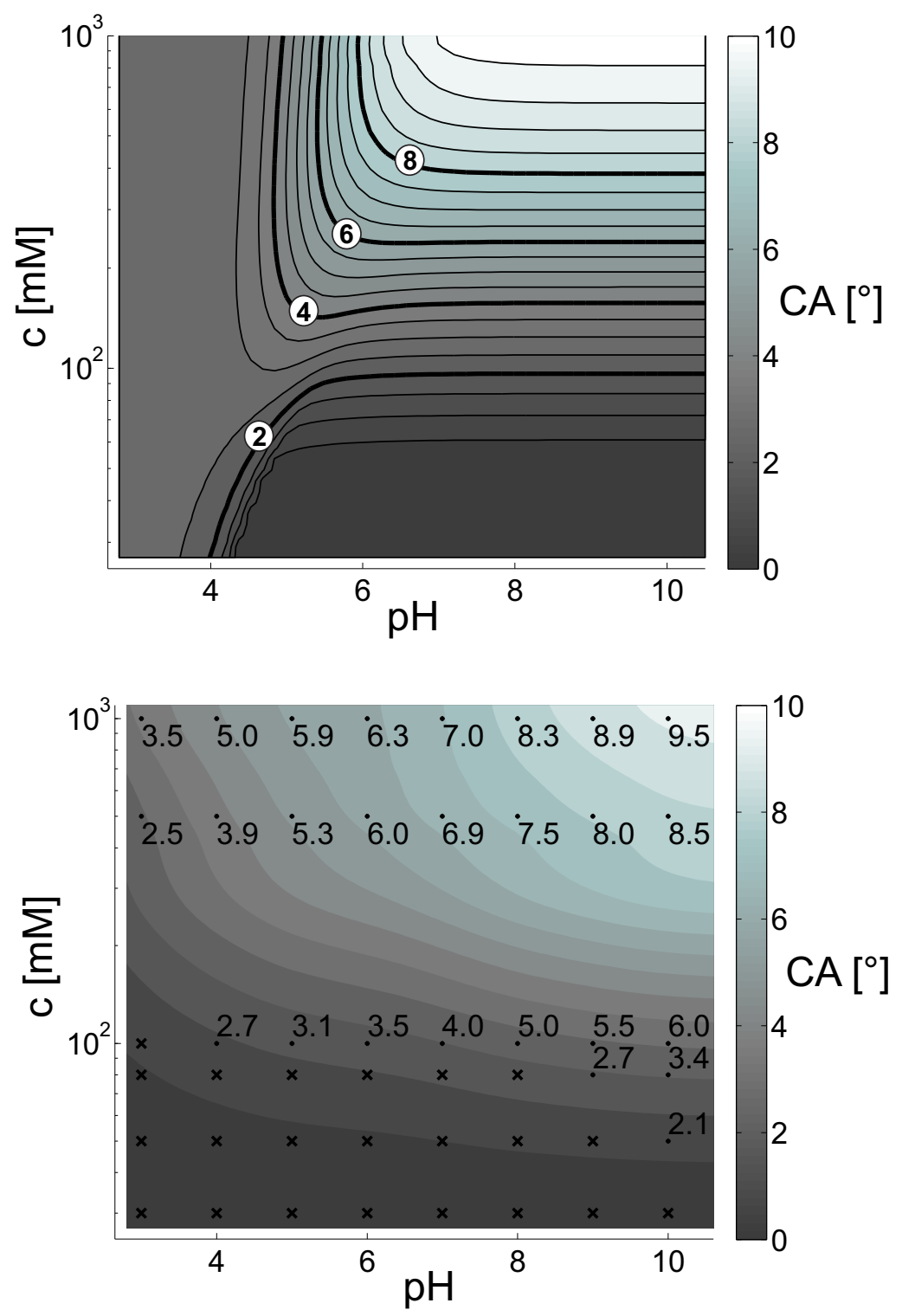

Figure 5.5: Gray scale encoded contact angle on mica in decane vs. $\mathrm{pH}$ and $\mathrm{CaCl}_{2}$ concentration. Top: model prediction with Constant Charge (CC) boundary condition; bottom: experimental data. Symbols $\left(\mathrm{x}: \theta<2^{\circ}\right)$ and numbers: experimental data same as Figure 4.2 with interpolated gray scale. Smoothed lines are guides to the eye based on the experimental datapoints. 


$$
\begin{gathered}
\frac{\partial^{2} \phi}{\partial z^{2}}=\kappa_{D}^{2} \phi \\
\left.\frac{\partial \phi}{\partial z}\right|_{z=0}=-\sigma_{1}\left(\phi_{1}\right) \\
\left.\frac{\partial \phi}{\partial z}\right|_{z=h}=\sigma_{2}\left(\phi_{2}\right)
\end{gathered}
$$

The choice of the Debye-Huckel approximation allows two dramatic simplifications of the problem. First, the equation keeps the same form for an arbitrary mixture of (m:n) monovalent or divalent electrolytes, as the contribution of the different species appears only through the ionic strength in $\kappa_{D}$. Secondly, it is easier to find a suitable ansatz for the electrostatic potential in the electrolyte between the interfaces,

$$
\phi(z)=A \exp \left(\kappa_{D} z-\kappa_{D} h\right)+B \exp \left(-\kappa_{D} z\right)
$$

Despite the significant simplification introduced by linearizing the PB equation, the $\mathrm{CR}$ boundary conditions prevent us from finding an analytic expression for the coefficients A and B. An order zero approximation would be to assume that each interface keep its charge at infinite separation, i.e. $\sigma_{i}(\phi)=\sigma_{i, \infty}$. This is a rather crude assumption, as it neglects any effect from the other interface. We thus take a further step, and consider the linearized approximation. We have already introduced the charge regulation (CR) boundary condition in the previous section: $\sigma_{s}=f\left(\phi_{s}\right)$ [7]. Now a linearized approximation of this relation can be written as: $\sigma_{s}(i)=$ $\left(S(i)-K(i) \phi_{s}(i)\right)[8]$, where $\mathrm{S} \& \mathrm{~K}$ are the two constants describing the regulating surface. Hence, the boundary conditions are given by:

$$
\begin{gathered}
-\left.\frac{\partial \phi}{\partial z}\right|_{z=0}=\left(\frac{S_{1}}{\epsilon \epsilon_{0}}-\frac{\left.K_{1} \phi\right|_{z=0}}{\epsilon \epsilon_{0}}\right) ; \\
\left.\frac{\partial \phi}{\partial z}\right|_{z=h}=\left(\frac{S_{2}}{\epsilon \epsilon_{0}}-\frac{\left.K_{2} \phi\right|_{z=h}}{\epsilon \epsilon_{0}}\right)
\end{gathered}
$$

where $S(i)=\sigma\left(\phi_{s, \infty}(i)\right)-\phi_{s, \infty}(i)\left[\frac{\partial \sigma(\phi)}{\partial \phi}\right]$ and $K(i)=-\left[\frac{\partial \sigma(\phi)}{\partial \phi}\right]\left(\phi_{s, \infty}\right.$ is the surface potential of an isolated surface in electrolyte, i.e., at infinite separation). 
Thus, solving Equation 5.12 with the bounday conditions in Equation 5.14a and Equation 5.14b (see Appendix), we obtain the electrostatic potential in the film:

$$
\begin{aligned}
\phi(z) & =\frac{\phi_{2, \infty}-\Delta_{2} \phi_{1, \infty} \exp \left(-\kappa_{D} h\right)}{1-\Delta_{1} \Delta_{2} \exp \left(-2 \kappa_{D} h\right)} \exp \left(-\kappa_{D}(h-z)\right) \\
& +\frac{\phi_{1, \infty}-\Delta_{1} \phi_{2,, \infty} \exp \left(-\kappa_{D} h\right)}{1-\Delta_{1} \Delta_{2} \exp \left(-2 \kappa_{D} h\right)} \exp \left(-\kappa_{D} z\right)
\end{aligned}
$$

where, the parameter $\Delta_{i}$ is given by $\frac{K_{i}-\kappa_{D} \epsilon \epsilon_{0}}{K_{i}+\kappa_{D} \epsilon_{0}}$ and surface potential of an isolated surface in electrolyte, $\phi_{i, \infty}$ is given by $\frac{S_{i}}{K_{i}+\kappa_{D} \epsilon \epsilon_{0}}$. Here, that $\Delta_{i}=1$ denotes the constant surface potential boundary condition and $\Delta_{i}=-1$ represents the constant surface charge boundary condition. The disjoining pressure in the film is given by:

$$
\begin{gathered}
\Pi_{e l}(z)=\frac{\epsilon \epsilon_{0}}{2}\left[\kappa_{D}^{2} \phi^{2}-\left(\frac{\partial \phi}{\partial z}\right)^{2}\right] \\
=2 \epsilon \epsilon_{0} \kappa_{D}^{2}\left[\frac{2 \phi_{1, \infty} \phi_{2, \infty} \exp \left(-\kappa_{D} h\right)-\left[\Delta_{1} \phi_{2, \infty}{ }^{2}+\Delta_{2} \phi_{1, \infty}{ }^{2}\right] \exp \left(-2 \kappa_{D} h\right)}{\left(1-\Delta_{1} \Delta_{2} \exp \left(-2 \kappa_{D} h\right)\right)^{2}}\right. \\
\left.-\frac{\phi_{1, \infty} \phi_{2, \infty} \exp \left(-\kappa_{D} h\right)}{1-\Delta_{1} \Delta_{2} \exp \left(-2 \kappa_{D} h\right)}\right]
\end{gathered}
$$

and the electrostatic contribution of the interaction potential is given by:

$$
\begin{aligned}
\Phi_{e l}(h) & =-\int_{\infty}^{h} \Pi_{e l}(x) d x \\
& =\epsilon \epsilon_{0} \kappa_{D}\left[\frac{2 \phi_{1, \infty} \phi_{2, \infty} \exp \left(-\kappa_{D} h\right)-\left[\Delta_{1} \phi_{2, \infty}^{2}+\Delta_{2} \phi_{1, \infty}{ }^{2}\right] \exp \left(-2 \kappa_{D} h\right)}{1-\Delta_{1} \Delta_{2} \exp \left(-2 \kappa_{D} h\right)}\right]
\end{aligned}
$$

One might ask if the linearization procedure is suitable for our experimental mineral-water-oil systems. In order to answer this question, we need to briefly discuss the structure of the $\sigma(\psi)$ curve for the interfaces under consideration. As we have described already in section 5.2, the surface chemistry of the solid-water interface is captured by the surface complexation modeling. In this model, each surface site $S$ can bind a proton $\mathrm{H}^{+}$or a cation $C^{Z_{c}}$. This results in a distinct profile for the $\sigma(\psi)$ function, which is 

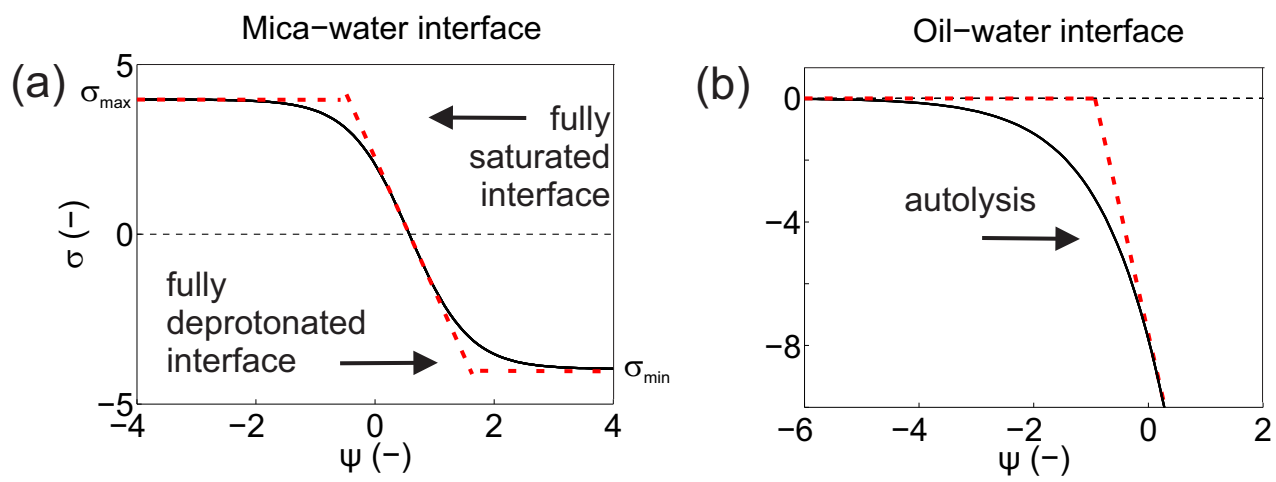

Figure 5.6: (a) $\sigma(\psi)$ profile for the mica-water interface, based on the complexation model. The adsorption coefficients are $p K_{H}=4$ and $p K_{C a^{2+}}=1.5$, for site density $\Gamma_{\text {mica }}=0.8$ sites $/ \mathrm{nm}^{2}$. (b) $\sigma(\psi)$ profile for the oil-water interface, based also on the complexation model. The autolysis reaction coefficient is $p K_{H}=7$, for site density $\Gamma_{\text {oil }}=17.3$ sites $/ \mathrm{nm}^{2}$. Both curves are obtained for $\mathrm{pH}=6$ and $\mathrm{Ca}^{2+}$ bulk concentration $\left[\mathrm{Ca}^{2+}\right]_{\infty}=100 \mathrm{mM}$.

sketched in Figure 5.6. $\sigma(\psi)$ shows two plateaus, corresponding to a fully deprotonated substrate, with charge $\sigma_{\min }$, and a fully saturated one, of charge $\sigma_{\max }$. As the dashed red line shows, this kind of behavior can be quite accurately captured by a piecewise linear approximation, with two constantcharge $(\Delta=-1)$ regions and a linear transition among them.

As explained in the previous chapter, as well as in the Model section 5.2 $[9,14,16]$, the surface charge at the oil-water interface can be described by enhanced water autolysis at the oil-water interface, described by equation:

$$
\mathrm{S} \equiv \mathrm{H}_{2} \mathrm{O} \rightleftharpoons \mathrm{S} \equiv \mathrm{OH}^{-}+\mathrm{H}^{+}
$$

resulting in the surface charge profiles shown in Figure 5.6b. According to this description, the oil-water interface is neutral at large negative potentials, and develops a negative charge for increasing positive potential. For both interfaces, the details of $\sigma(\psi)$ depend on the specific chemistry of the surface, i.e. the equilibrium constant for each absorption/desorption reaction. However, the character of these curves, with plateaus connected by sharp transitions, is rather universal, and can be traced back to the Langmuir adsorption theory (described in chapter 2) underlying these surface chemistry models. It is worth stressing the importance of this observation. Generally speaking, a linearized approach is only valid for small perturbations to equilibrium, with an infinite separation value charge density $\sigma_{\infty}$. However, the specific nature of the interfaces we consider allow us to employ a piecewise 
linear description over the whole range of electrostatic potential. Here the value of $\sigma_{\infty}$ then determines which linear regime is relevant for the system.

\subsubsection{Wetting Phase-Space Analysis}

In Chapter 2, we discussed four distinct wetting configurations for a solidfluid-fluid (rock-water-oil) system. These are: complete wetting $(\mathrm{CW})$, partial wetting $(\mathrm{PW})$, pseudo-partial wetting $(\mathrm{PP})$ and metastable wetting $(\mathrm{M})$. In the following section, we will investigate the implication of our linearized system for these various wetting configurations. We will consider the wetting behavior of a solid-water-oil system only as a function of the charge regulation parameter $\Delta$ and the surface charge of the interface at infinite separation $\psi_{s i, \infty}$.

First, we rewrite the disjoining pressure in non-dimensional form as:

$$
\begin{aligned}
\Pi_{e l}(h) & =4 \frac{e^{-\kappa_{D} h}}{\left(1-\Delta_{1} \Delta_{2} e^{-2 \kappa_{D} h}\right)^{2}} \\
& {\left[\psi_{1, \infty} \psi_{2, \infty}+\Delta_{1} \Delta_{2} \psi_{1, \infty} \psi_{2, \infty} e^{-2 \kappa_{D} h}-\left[\Delta_{1} \psi_{2, \infty}^{2}+\Delta_{2} \psi_{1, \infty}^{2}\right] e^{-\kappa_{D} h}\right] . }
\end{aligned}
$$

The sign of $\Pi_{e l}(h)$ is uniquely defined by the term in square brackets, which is a quadratic polynomial in $x=\exp \left(-\kappa_{D} h\right)$ :

$$
\pi(x)=\left[\psi_{1, \infty} \psi_{2, \infty}+\Delta_{1} \Delta_{2} \psi_{1, \infty} \psi_{2, \infty} x^{2}-\left[\Delta_{1} \psi_{2, \infty}^{2}+\Delta_{2} \psi_{1, \infty}^{2}\right] x\right] .
$$

The roots of $\pi(x)$ are $x_{ \pm}=\left\{\frac{\psi_{2, \infty}}{\Delta_{2} \psi_{1, \infty}}, \frac{\psi_{1, \infty}}{\Delta_{1} \psi_{2, \infty}}\right\}$. The corresponding equilibrium film thicknesses are thus $h_{ \pm}=\left\{\kappa_{D}^{-1} \ln \frac{\Delta_{2} \psi_{1, \infty}}{\psi_{2, \infty}}, \kappa_{D}^{-1} \ln \frac{\Delta_{1} \psi_{2, \infty}}{\psi_{1, \infty}}\right\}$. This information allows us to draw some very general conclusions. If either $h_{+}>0$ or $h_{-}>0$, the interface potential exhibits a local extremum. Note that both solutions cannot be positive at the same time, as it would imply $\Delta_{1} \Delta_{2}>1$, which is impossible by construction. The interface potential will thus show at most one local maximum or minimum. As $\Phi_{e l}(\infty)=0$, it is sufficient to check the sign of the potential at $h=0$ to distinguish between the two. If neither $h_{+}$nor $h_{-}$is positive, $\Phi_{e l}(h)$ is monotonously attractive or repulsive. Again, it is sufficient to check the sign of $\Phi_{e l}(0)$ to discriminate the latter two scenarios. 


\subsubsection{Constant Charge and Constant Charge-like Inter- faces $-\Delta_{1,2}<0$}

$$
\Delta_{1}:-1 \Delta_{2}:-1
$$

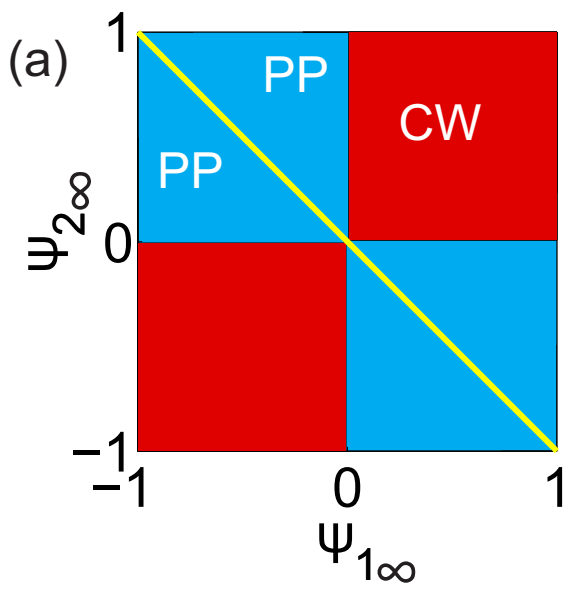

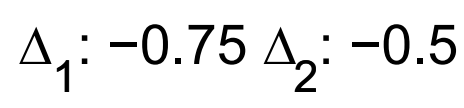

(b)

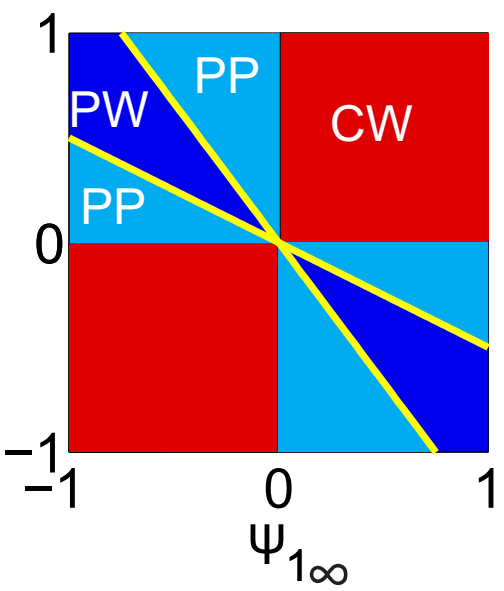

Figure 5.7: Phase-space maps for a) $\Delta_{1}=\Delta_{2}=-1$, and b) $\Delta_{1}=-0.75$, $\Delta_{2}=-0.5$. The color coding: red for complete wetting (CW), dark blue for partial wetting (PW) and light blue for pseudo-partial wetting (PP).

We first consider the case $\Delta_{1,2}<0$. As an example, Figure 5.7 shows the possible wetting configuration for $\psi_{1, \infty}, \psi_{2, \infty} \in[-1,1]$, when (a) $\Delta_{1}=$ $\Delta_{2}=-1$, and (b) $\Delta_{1}=-0.75, \Delta_{1}=-0.5$. If the charge of the two substrates at infinite separation has the same $\operatorname{sign}, \operatorname{sign}\left(\psi_{1 \infty}\right)=\operatorname{sign}\left(\psi_{2 \infty}\right)$, one can easily check that $\Pi_{e l}(h)$ is always positive. The interaction of the interfaces is thus always repulsive, leading to a complete wetting configuration. If $\operatorname{sign}\left(\psi_{1, \infty}\right) \neq \operatorname{sign}\left(\psi_{2, \infty}\right), \Phi_{e l}(h)$ shows a local minimum for $\psi_{1, \infty}>\psi_{2, \infty} / \Delta_{2}$ and $\psi_{1, \infty}<\Delta_{1} \psi_{2, \infty}$. For $\Delta_{1} \psi_{2, \infty}<\psi_{1, \infty}<\psi_{2, \infty} / \Delta_{2}$, the interface potential is monotonously attractive, leading to a partial wetting configuration. We can get a physical picture of these results starting from the extreme case of constant charge at both interfaces, $\Delta_{1,2}=-1$. In this case, the surface charge of each interface is not affected by the proximity of the other one. This explain the repulsion between interfaces of same-sign surface charge, and long-range attraction between opposite-sign interfaces. However, when surface charges of different magnitude and sign are brought together, a net charge will be localized between them, leading to a strong imbalance of the local ionic concentration. Osmotic pressure will thus oppose the fur- 
ther approach of the interfaces, generating a strong short-range repulsion. For this reason, constant charge interfaces exhibit partial wetting only when $\psi_{1, \infty}=-\psi_{2, \infty}$. In this case, the two surface charges match exactly, leading to zero net charge when the interfaces are brought together. For the more general case $\Delta_{1,2}<0$, a wider region of complete wetting configurations appear, as the interfaces will charge-regulate to match their magnitude at short separation, in order to avoid the energy-expensive configuration with localized charge in a thin film. Whether the charge regulation results in a pseudo-partial or partial wetting configuration depends on the $\Delta_{1,2}$ and the imbalance $\psi_{1, \infty}-\psi_{2, \infty}$. Large imbalances, and deltas close to one, lead to a stronger short range repulsion.

\subsubsection{Constant Potential and Constant Potential-like In- terfaces $-\Delta_{1,2}>0$}
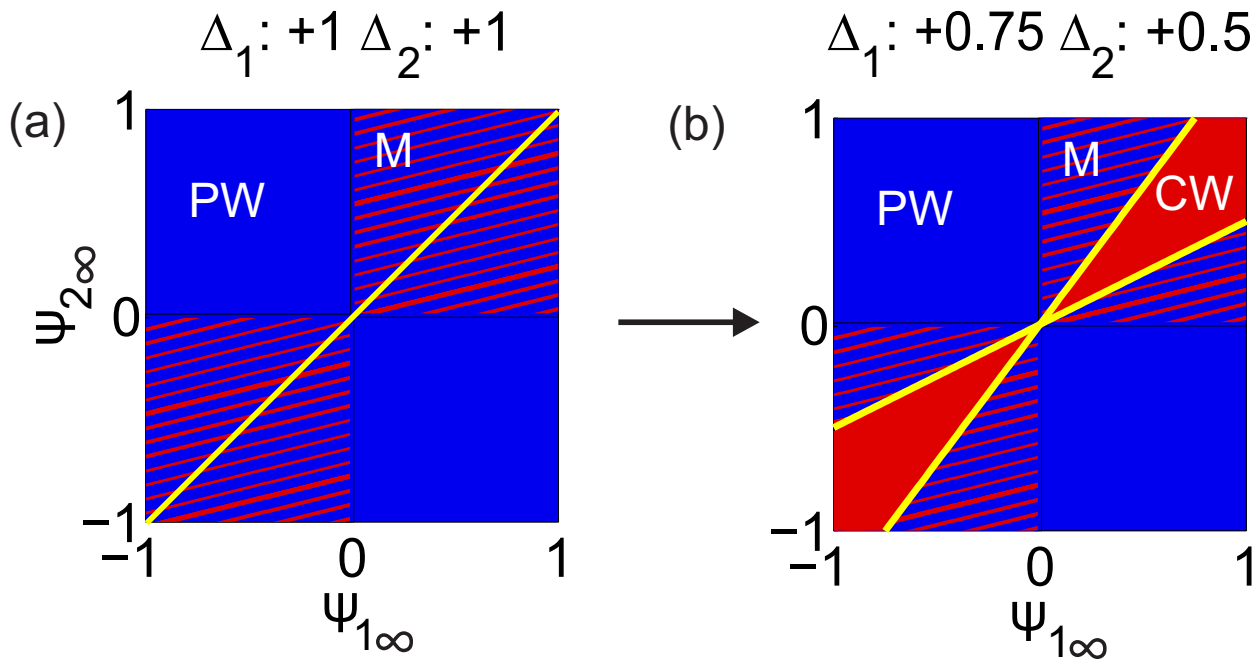

Figure 5.8: Phase-space maps for a) $\Delta_{1}=\Delta_{2}=+1$, and b) $\Delta_{1}=+0.75$, $\Delta_{2}=+0.5$. The color coding : red for complete wetting (CW), dark blue for partial wetting $(\mathrm{PW})$, and red-blue stripes for a metastable wetting state $(\mathrm{M})$.

If the potential of the two substrates at infinite separation has the opposite sign, we see that $\Pi_{e l}(h)$ is always negative. The interaction of the interfaces is thus always attractive, leading to a partial wetting configuration. If the charge of the interfaces at infinite separation has the same sign, we observe a local maximum in $\Phi(h)$ for $\psi_{1, \infty}>\psi_{2, \infty} / \Delta_{2}$ and $\psi_{1, \infty}<\Delta_{1} \psi_{2, \infty}$. 
As discussed in relation to the various wetting configurations in Chapter 2 , this range correspond to a metastable wetting configuration, which can evolve into a partial or complete wetting state depending on the initial thickness of the film. For $\Delta_{1} \psi_{2, \infty}<\psi_{1 \infty}<\psi_{2, \infty} / \Delta_{2}$, the interface potential is monotonously repulsive, leading to a complete wetting configuration.

Once again, we can get a physical understanding of these results starting from the constant potential case $\Delta_{1,2}=1$ (Figure 5.8a), and generalizing some trends for $1>\Delta_{1,2}>0$ (Figure 5.8b). It is convenient to discuss the long $\left(\kappa_{D} h \gg 1\right)$ and short $\left(\kappa_{D} h \ll 1\right)$ range interactions separately. For $\kappa_{D} h \gg 1$, the character of the interface potential is largely defined by the sign of the surface charge at infinite separation. We thus observe attraction for opposite-sign surface charges, and repulsion for same-sign ones. For $\kappa_{D} h \ll 1$, the electrolyte screening is much less relevant. The two interfaces are kept at constant potential, and thus act like the plates of a capacitor. If $\psi_{1, \infty} \neq \psi_{2, \infty}$, an electric field develops between them, whose intensity diverges while the interfaces get closer. This leads to a diverging attractive interaction for any case but $\psi_{1 \infty}=\psi_{2 \infty}$. Once again, charge regulation "softens" this behavior. In Figure 5.8b we plot the possible wetting configurations for for $\psi_{1, \infty}, \psi_{2, \infty} \in[-1,1]$, when $\Delta_{1}=0.75$, and $\Delta_{2}=0.5$. We observe that a complete-wetting branch opens around the $\psi_{1 \infty}=\psi_{2 \infty}$ line. This is because the charge at the interfaces regulates to reduce the strength of the electric field, and thus avoid the divergent behavior of the constantpotential case. Whether this leads to a finite attractive interaction at short range, or even a repulsive one depends again on the $\Delta_{1,2}$ and the imbalance $\psi_{1 \infty}-\psi_{2 \infty}$.

\subsubsection{Comparison of Two Approaches}

First, we have investigated the influence of charge reversal on various wetting configurations, based on constant charge (CC) boundary conditions. The main outcome from this approach is that if there is a charge-reversal in one of the two interfaces, then the wetting configuration switches from complete wetting (CW) to pseudo partial wetting (PP). When we consider linearized charge regulation (CR) boundary conditions, we discover a richer scenario of wetting configurations. As seen from the wetting phase-space diagrams, opposite charges at the interfaces might lead to a partial wetting regime or a pseudo partial regime with a molecularly thin adsorbed film present next to the macroscopic drop (our experimental cases fall in this cat- 
egory). In a constant potential-like approach within the charge regulation boundary conditions, even two interfacial charges with same sign might lead to a metastable $(\mathrm{M})$ wetting configuration, which could be a complete wetting $(\mathrm{CW})$ situation or not. We observe that contact angles calculated by the charge regulation $(\mathrm{CR})$ approach fit well at relatively low $\mathrm{pH}$ (up to $\mathrm{pH}$ 6), and thereafter diverge from the experimental observations. However, the essence of charge reversal remains the same in both the approaches: as the sign of the surface charge changes in any one of the interfaces, there is a transition in the phase diagram which leads to a macroscopically visible change in the wetting configuration.

\subsection{Concluding Remarks}

In this chapter, we described the electrostatic contribution to the wetting properties of a specific type of mineral-water-oil systems, first using a constant charge approach and later introducing a linearized charge regulation model. We start with a typical complexation model to emulate the surface chemistry of an adsorbing interface which is then coupled with the PB model. When we numerically solve the PB model with constant charge boundary conditions, the importance of charge-reversal at one of the interfaces upon wetting transition becomes evident. Using the linearized charge regulation model, we predict four distinct wetting regimes, which are functions of the potential at infinite separation and a charge regulation parameter $\Delta$ for each interface. Using these boundary parameters as input, our approach provides analytic expressions for the equilibrium contact angle in the partial wetting configurations, and the boundaries between wetting states. We observe that charge regulation "relaxes" the asymptotic repulsive/attractive behavior that would arise for pure constant charge or constant potential boundary conditions.

Our modelling clearly shows that electrostatic contributions play a major role in adsorption induced wetting. But other contributions such as van der Waal's or hydration effects are not investigated in detail in this model. Ionspecific effects have an enormous contribution in the colloidal regime where the charged species of a system behave in very special ways depending on the dipole effects, polarizability effects etc. In the following chapter, we will experimentally investigate the ion-specific effects upon adsorption and how they influence the wetting picture we have drawn so far. 


\subsection{Appendix}

\subsubsection{Non-dimensionalization of Charge Regulation Model}

We have obtained the expression of surface charge using the chemical adsorption reactions in Equation 5.6. We use the non-dimensional version of Equation 5.6 for our following calculations:

$$
\sigma_{s}(n d)=\frac{e \Gamma\left(\frac{\left(Z_{c}-1\right)\left[C^{\left(Z_{c}\right)+}\right]_{\infty} \exp \left(-\frac{Z_{c} e \phi_{s}}{k_{B} T}\right)}{K_{C}}-1\right)}{1+\frac{\left[H^{+}\right]_{\infty} \exp \left(-\frac{e \phi_{s}}{k_{B} T}\right)}{K_{H}}+\frac{\left[C^{\left(Z_{c}\right)+}\right]_{\infty} \exp \left(-\frac{Z_{c} e \phi_{s}}{k_{B} T}\right)}{K_{C}}} * \frac{e}{\epsilon \epsilon_{0} k_{B} T \kappa_{D}}
$$

where, we call $\frac{e}{\epsilon \epsilon_{0} k_{B} T k_{D}}=\sigma^{*}$, the non-dimensionalizing factor for the surface charge.

In the context of the calculation of the electrostatic potential in the thin aqueous film, we have introduced the linear PB equation in Equation 5.12. Written in non-dimensional terms with $\psi=\frac{e \phi}{k_{B} T}$ and $\zeta=\kappa_{D} h$ :

$$
\frac{\partial^{2} \psi}{\partial \zeta^{2}}=\psi
$$

In the following calculations, the non-dimensionalizing parameter for surface potential $\left(e \phi / k_{B} T\right)^{-1}$ will be written as $\phi^{*}$.

The linearized charge regulation boundary conditions in non-dimensional form are:

$$
\begin{gathered}
-\left.\frac{\partial \psi}{\partial \zeta}\right|_{\zeta=0}=\frac{S_{1}}{\sigma *}-\left.\frac{\phi^{*}}{\sigma^{*}} K_{1} \psi\right|_{\zeta=0} ; \\
\left.\frac{\partial \psi}{\partial \zeta}\right|_{\zeta=\kappa_{D} h}=\frac{S_{2}}{\sigma *}-\left.\frac{\phi^{*}}{\sigma^{*}} K_{2} \psi\right|_{\zeta=\kappa_{D} h}
\end{gathered}
$$

where $S(i)=\sigma\left(\phi_{s, \infty}(i)\right)-\phi_{s, \infty}(i)\left[\frac{\partial \sigma(\phi)}{\partial \phi}\right]$ and $K=-\left[\frac{\partial \sigma(\phi)}{\partial \phi}\right]\left(\phi_{s, \infty}\right.$ is the surface potential of an isolated surface in electrolyte). In the following section, we describe the solution of Equation 5.22 with these boundary conditions described in Equation 5.23a and 5.23b. 


\subsubsection{Calculation of Electrostatic Potential}

We begin with an estimate (aansatz) for the solution of the differential equation Equation 5.22:

$$
\psi(\zeta)=A \exp \left(\zeta-\kappa_{D} h\right)+B \exp (-\zeta)
$$

Hence, at the mineral-water interface (where, $\zeta=0$ ) and at the oil-water interface $\left(\zeta=\kappa_{D} h\right)$, we obtain:

$$
\begin{gathered}
\left.\psi\right|_{\zeta=0}=A \exp \left(-\kappa_{D} h\right)+B ; \\
\left.\psi\right|_{\zeta=\kappa_{D} h}=A+B \exp \left(-\kappa_{D} h\right)
\end{gathered}
$$

Replacing Equation 5.25a in 5.23a, we obtain:

$$
\begin{aligned}
\left.\frac{\partial\left[A \exp \left(\zeta-\kappa_{D} h\right)+B \exp (-\zeta)\right]}{\partial \zeta}\right|_{\zeta=0} & =-\frac{S_{1}}{\sigma *}+\frac{\phi^{*}}{\sigma^{*}} K_{1}\left[A \exp \left(-\kappa_{D} h\right)+B\right] \\
{\left.\left[A \exp \left(\zeta-\kappa_{D} h\right)-B \exp (-\zeta)\right]\right|_{\zeta=0} } & =-\frac{S_{1}}{\sigma *}+\frac{\phi^{*}}{\sigma^{*}} K_{1}\left[A \exp \left(-\kappa_{D} h\right)+B\right] \\
{\left[A \exp \left(-\kappa_{D} h\right)-B\right] } & =-\frac{S_{1}}{\sigma *}+\frac{\phi^{*}}{\sigma^{*}} K_{1} A \exp \left(-\kappa_{D} h\right)+\frac{\phi^{*}}{\sigma^{*}} K_{1} B
\end{aligned}
$$

which provides the relation:

$$
A \exp \left(-\kappa_{D} h\right)\left[\frac{\phi^{*}}{\sigma^{*}} K_{1}-1\right]+B\left[\frac{\phi^{*}}{\sigma^{*}} K_{1}+1\right]=\frac{S_{1}}{\sigma^{*}}
$$

Similarly, from Equation 5.25b and 5.23b, we obtain,

$$
A\left[\frac{\phi^{*}}{\sigma^{*}} K_{2}+1\right]+B \exp \left(-\kappa_{D} h\right)\left[\frac{\phi^{*}}{\sigma^{*}} K_{2}-1\right]=\frac{S_{2}}{\sigma^{*}}
$$

Hence, Equation 5.26a-Equation 5.26b $\times \frac{\frac{\phi^{*}}{\sigma^{*}} K_{1}-1}{\frac{\phi^{*}}{\sigma^{*}} K_{1}+1}$ and subsequent rearranging gives,

$$
B=\frac{\frac{S_{1}}{\sigma^{*}}\left(\frac{\phi^{*}}{\sigma^{*}} K_{2}+1\right)-\frac{S_{2}}{\sigma^{*}}\left(\frac{\phi^{*}}{\sigma^{*}} K_{1}-1\right)}{\left(\frac{\phi^{*}}{\sigma^{*}} K_{1}+1\right)\left(\frac{\phi^{*}}{\sigma^{*}} K_{2}+1\right)-\left(\frac{\phi^{*}}{\sigma^{*}} K_{1}-1\right)\left(\frac{\phi^{*}}{\sigma^{*}} K_{2}-1\right) \exp \left(-2 \kappa_{D} h\right)}
$$


and similarly, Equation 5.26a $\times \frac{\frac{\phi^{*}}{\sigma^{*}} K_{2}-1}{\frac{\phi^{*}}{\sigma^{*}} K_{2}+1}$-Equation 5.26b and subsequent rearranging gives,

$$
A=\frac{\frac{S_{1}}{\sigma^{*}}\left(\frac{\phi^{*}}{\sigma^{*}} K_{2}-1\right)-\frac{S_{2}}{\sigma^{*}}\left(\frac{\phi^{*}}{\sigma^{*}} K_{1}+1\right)}{\left(\frac{\phi^{*}}{\sigma^{*}} K_{1}-1\right)\left(\frac{\phi^{*}}{\sigma^{*}} K_{2}-1\right) \exp \left(-2 \kappa_{D} h\right)-\left(\frac{\phi^{*}}{\sigma^{*}} K_{1}+1\right)\left(\frac{\phi^{*}}{\sigma^{*}} K_{2}+1\right)}
$$

Thus, we can rewrite Equation 5.24 as:

$$
\begin{array}{ll}
\psi(\zeta) & = \\
& \frac{\frac{S_{1}}{\sigma^{*}} \exp \left(-\kappa_{D} h\right)\left(\frac{\phi^{*}}{\sigma^{*}} K_{2}-1\right)-\frac{S_{2}}{\sigma^{*}}\left(\frac{\phi^{*}}{\sigma^{*}} K_{1}+1\right)}{\left(\frac{\phi^{*}}{\sigma^{*}} K_{1}-1\right)\left(\frac{\phi^{*}}{\sigma^{*}} K_{2}-1\right) \exp \left(-2 \kappa_{D} h\right)-\left(\frac{\phi^{*}}{\sigma^{*}} K_{1}+1\right)\left(\frac{\phi^{*}}{\sigma^{*}} K_{2}+1\right)} \exp \left(\zeta-\kappa_{D} h\right) \\
& +\frac{\frac{S_{1}}{\sigma^{*}}\left(\frac{\phi^{*}}{\sigma^{*}} K_{2}+1\right)-\frac{S_{2}}{\sigma^{*}} \exp \left(-\kappa_{D} h\right)\left(\frac{\phi^{*}}{\sigma^{*}} K_{1}-1\right)}{\left(\frac{\phi^{*}}{\sigma^{*}} K_{1}+1\right)\left(\frac{\phi^{*}}{\sigma^{*}} K_{2}+1\right)-\left(\frac{\phi^{*}}{\sigma^{*}} K_{1}-1\right)\left(\frac{\phi^{*}}{\sigma^{*}} K_{2}-1\right) \exp \left(-2 \kappa_{D} h\right)} \exp (-\zeta)
\end{array}
$$

or, we can write,

$$
\begin{gathered}
\psi(\zeta)=\frac{S_{1}^{*} \exp \left(-\kappa_{D} h\right)\left(K_{2}^{*}-1\right)-S_{2}^{*}\left(K_{1}^{*}+1\right)}{\left(K_{1}^{*}-1\right)\left(K_{2}^{*}-1\right) \exp \left(-2 \kappa_{D} h\right)-\left(K_{1}^{*}+1\right)\left(K_{2}^{*}+1\right)} \exp \left(\zeta-\kappa_{D} h\right) \\
+\frac{S_{1}^{*}\left(K_{2}^{*}+1\right)-S_{2}^{*} \exp \left(-\kappa_{D} h\right)\left(K_{1}^{*}-1\right)}{\left(K_{1}^{*}+1\right)\left(K_{2}^{*}+1\right)-\left(K_{1}^{*}-1\right)\left(K_{2}^{*}-1\right) \exp \left(-2 \kappa_{D} h\right)} \exp (-\zeta)
\end{gathered}
$$

where, $K_{i}^{*}=\frac{\partial \sigma_{n d}}{\partial \psi}=K_{i} \frac{\phi^{*}}{\sigma^{*}}$ and $S_{i}^{*}=\frac{S_{i}}{\sigma^{*}}$. We also recall the definition of $\phi_{\infty}$ from section 4.2: $\phi_{i, \infty}=\frac{S_{i}}{K_{i}+\kappa_{D} \epsilon \epsilon_{0}}$, which we can simplify as following: 


$$
\begin{aligned}
\phi_{i, \infty} & =\frac{S_{i}}{K_{i}+\kappa_{D} \epsilon \epsilon_{0}} \\
\Longrightarrow \psi_{i, \infty} & =\left(\frac{e}{k_{B} T}\right) \frac{S_{i}}{\frac{\partial \sigma}{\partial \phi}+\kappa_{D} \epsilon \epsilon_{0}} \\
\Longrightarrow \psi_{i, \infty} & =\left(\frac{e}{k_{B} T}\right) \frac{S_{i}}{\frac{\partial \sigma_{n d}}{\partial \psi} \frac{\sigma^{*}}{\phi^{*}}+\kappa_{D} \epsilon \epsilon_{0}} \\
\Longrightarrow \psi_{i, \infty} & =\frac{\frac{S_{i}}{\epsilon \epsilon_{0} k_{B} T \kappa_{D} / e}}{K_{i}^{*}+1}
\end{aligned}
$$

which gives the relation $\psi_{i, \infty}=\frac{S_{i}^{*}}{K_{i}^{*}+1}$. At the same time, we also recall that: $\Delta_{i}=\frac{K_{i}-\kappa_{D} \epsilon \epsilon_{0}}{K_{i}+\kappa_{D} \epsilon \epsilon_{0}}$, which then, by definition of $K_{i}^{*}$ can be written as: $\Delta_{i}=$ $\frac{K_{i}^{*}-1}{K_{i}^{*}+1}$. Now, we focus on Equation 5.30 again, and rewrite the expression as:

$$
\begin{aligned}
\psi(\zeta) & =\frac{\frac{S_{2}^{*}}{\left(K_{2}^{*}+1\right)}-\frac{S_{1}^{*}}{K_{1}^{*}+1} \frac{\left(K_{2}^{*}-1\right)}{K_{2}^{*}+1} \exp \left(-\kappa_{D} h\right)}{1-\frac{\left(K_{1}^{*}-1\right)\left(K_{2}^{*}-1\right)}{\left(K_{1}^{*}+1\right)\left(K_{2}^{*}+1\right)} \exp \left(-2 \kappa_{D} h\right)} \exp \left(\zeta-\kappa_{D} h\right) \\
& +\frac{\frac{S_{1}^{*}}{\left(K_{1}^{*}+1\right)}-\frac{S_{2}^{*}}{K_{2}^{*}+1} \frac{\left(K_{1}^{*}-1\right)}{K_{1}^{*}+1} \exp \left(-\kappa_{D} h\right)}{1-\frac{\left(K_{1}^{*}-1\right)\left(K_{2}^{*}-1\right)}{\left(K_{1}^{*}+1\right)\left(K_{2}^{*}+1\right)} \exp \left(-2 \kappa_{D} h\right)} \exp (-\zeta)
\end{aligned}
$$

We can rewrite Eq.5.31 as following:

$$
\begin{aligned}
\psi(\zeta) & =\frac{\psi_{2, \infty}-\Delta_{2} \psi_{1, \infty} \exp \left(-\kappa_{D} h\right)}{1-\Delta_{1} \Delta_{2} \exp \left(-2 \kappa_{D} h\right)} \exp \left(\zeta-\kappa_{D} h\right) \\
& +\frac{\psi_{1, \infty}-\Delta_{1} \psi_{2, \infty} \exp \left(-\kappa_{D} h\right)}{1-\Delta_{1} \Delta_{2} \exp \left(-2 \kappa_{D} h\right)} \exp (-\zeta)
\end{aligned}
$$

which is the non-dimensional equivalent of Equation 5.17. Just as in the case of Equation 5.17, we note here, that $\Delta_{i}=1$ denotes the constant surface potential boundary condition and $\Delta_{i}=-1$ represents the constant surface charge boundary condition.

\subsubsection{Disjoining Pressure and Interaction Potential}

Now, the disjoining pressure is calculated by: 


$$
\begin{aligned}
\Pi_{e l-n d}(\zeta) & =\psi^{2}-\left(\frac{\partial \psi}{\partial \zeta}\right)^{2} \\
B u t, \psi(\zeta) & =X \exp \left(\zeta-\kappa_{D} h\right)+Y \exp (-\zeta) \\
\text { or, } \frac{\partial \psi}{\partial \zeta} & =X \exp \left(\zeta-\kappa_{D} h\right)-Y \exp (-\zeta)
\end{aligned}
$$

where $X$ and $Y$ are prefactors from Eq.7.30. Hence, we can write:

$$
\begin{aligned}
& \Pi_{e l-n d}(\zeta)=4 X Y \exp \left(-\kappa_{D} h\right) \\
& =4 \frac{\left[\psi_{2, \infty}-\Delta_{2} \psi_{1, \infty} \exp \left(-\kappa_{D} h\right)\right]}{1-\Delta_{1} \Delta_{2} \exp \left(-2 \kappa_{D} h\right)} \frac{\left[\psi_{1, \infty}-\Delta_{1} \psi_{2, \infty} \exp \left(-\kappa_{D} h\right)\right]}{1-\Delta_{1} \Delta_{2} \exp \left(-2 \kappa_{D} h\right)} \exp \left(-\kappa_{D} h\right) \\
& =4\left[\frac{2 \psi_{1, \infty} \psi_{2, \infty} \exp \left(-\kappa_{D} h\right)-\left[\Delta_{1} \psi_{2, \infty}{ }^{2}+\Delta_{2} \psi_{1, \infty}\right] \exp \left(-2 \kappa_{D} h\right)}{\left(1-\Delta_{1} \Delta_{2} \exp \left(-2 \kappa_{D} h\right)\right)^{2}}\right. \\
& \left.-\frac{\psi_{1, \infty} \psi_{2, \infty} \exp \left(-\kappa_{D} h\right)}{1-\Delta_{1} \Delta_{2} \exp \left(-2 \kappa_{D} h\right)}\right]
\end{aligned}
$$

Subsequently, the electrostatic contribution of the interaction potential between the interfaces (non-dimensional form) is given by:

$$
\begin{gathered}
\Phi_{e l-n d}(\zeta)=-\int_{\infty}^{h} \Pi_{e l-n d}(\zeta) d \zeta \\
=2\left[\frac{2 \psi_{1, \infty} \psi_{2, \infty} \exp \left(-\kappa_{D} h\right)-\left[\Delta_{1} \psi_{2, \infty}{ }^{2}+\Delta_{2} \psi_{1, \infty}{ }^{2}\right] \exp \left(-2 \kappa_{D} h\right)}{1-\Delta_{1} \Delta_{2} \exp \left(-2 \kappa_{D} h\right)}\right.
\end{gathered}
$$




\section{Bibliography}

[1] F. Mugele, B. Bera, A. Cavalli, I. Siretanu, A. Maestro, M. Duits, M. A. Cohen-Stuart, D. van den Ende, I. Stocker, and I. Collins. Ion adsorption-induced wetting transition in oil-water-mineral systems. Scientific Reports, 5:10519, 2015.

[2] J. Lyklema. Fundamentals of Interface and Collloid Science, Volume I : Fundamentals. Academic Press, London, 2000.

[3] R. Hogg, T. W. Healy, and D. W. Fuerstenau. Mutual coagulation of colloidal dispersions. Transactions of the Faraday Society, 62(0):1638-1651, 1966.

[4] V. A. Parsegian and D. Gingell. On the electrostatic interaction across a salt solution between two bodies bearing unequal charges. Biophysical Journal, 12(9):1192-1204, 1972.

[5] D. Ben-Yaakov, D. Andelman, and H. Diamant. Interaction between heterogeneously charged surfaces: Surface patches and charge modulation. Physical Review E, 87(2):022402, 2013. PRE.

[6] S. H. Behrens and M. Borkovec. Electrostatic interaction of colloidal surfaces with variable charge. The Journal of Physical Chemistry B, 103(15):2918-2928, 1999.

[7] D. McCormack, S. L. Carnie, and D. Y. C. Chan. Calculations of electric double-layer force and interaction free energy between dissimilar surfaces. Journal of Colloid and Interface Science, 169(1):177-196, 1995.

[8] S. L. Carnie and D. Y. C. Chan. Interaction free energy between plates with charge regulation: A linearized model. Journal of Colloid and Interface Science, 161(1):260-264, 1993.

[9] J. K. Beattie. The intrinsic charge on hydrophobic microfluidic substrates. Lab on a Chip, 6(11):1409-1411, 2006. 
[10] P. J. Scales, F. Grieser, and T. W. Healy. Electrokinetics of the muscovite mica-aqueous solution interface. Langmuir, 6(3):582-589, 1990.

[11] S. Loh and S. P. Jarvis. Visualization of ion distribution at the micaelectrolyte interface. Langmuir, 26(12):9176-9178, 2010.

[12] P. Kekicheff, S. Marcelja, T.J. Senden, and V.E. Shubin. Charge reversal seen in electrical double layer interaction of surfaces immersed in 2:1 calcium electrolyte. Jour. Chem. Phys., 99:6098, 1993.

[13] M. Zembala and Z. Adamczyk. Measurements of streaming potential for mica covered by colloid particles. Langmuir, 16(4):1593-1601, 2000.

[14] J. Luetzenkirchen, T. Preocanin, and N. Kallay. A macroscopic water structure based model for describing charging phenomena at inert hydrophobic surfaces in aqueous electrolyte solutions. Phy. Chem. Chem. Phys., 10:4946, 2008.

[15] J.I. Kilpatrick, S-H. Loh, and S.P. Jarvis. Directly probing the effects of ions on hydration forces at interfaces. J. Am. Chem. Soc., 135:2628, 2013.

[16] M. Chaplin. Theory vs experiment: what is the surface charge of water. Water, 1:1-28, 2009.

[17] D. Bonn, J. Eggers, J. Indekeu, J. Meunier, and E. Rolley. Wetting and spreading. Reviews of Modern Physics, 81(2):739-805, 2009.

[18] I. Ibagon, M. Bier, and S. Dietrich. Wetting in electrolyte solutions. The Journal of Chemical Physics, 138(21):214703, 2013.

[19] I. Ibagon, M. Bier, and S. Dietrich. Order of wetting transitions in electrolyte solutions. The Journal of Chemical Physics, 140(17):174713, 2014. 


\section{CHAPTER 6}

\section{Cationic Hofmeister Series of Wettability in Mica-water-alkane Systems}

\section{Abstract}

The specific interaction of ions with macromolecules and solid-liquid interfaces is of crucial importance to many processes in biochemistry, colloid science, and engineering, as first pointed out by Hofmeister in the context of (de)stabilization of protein solutions. Here, we use contact angle goniometry to demonstrate that the macroscopic wettability of aqueous salt solutions of variable $\mathrm{pH}$ on mica immersed in ambient alkane increases from near-zero contact angles to values exceeding $10^{\circ}$, depending on the specific cation. This results in a series of increasing power to induce partial wetting in the order $\mathrm{Na}^{+}, \mathrm{K}^{+}<\mathrm{Li}^{+}<\mathrm{Rb}^{+}<\mathrm{Cs}^{+}<\mathrm{Ca}^{2+}<$ $\mathrm{Mg}^{2+}<\mathrm{Ba}^{2+}$, very similar to the direct Hofmeister series for proteins. Complementary Atomic Force Microscopy measurements show that charge reversal of the mica-electrolyte interfaces promotes finite contact angles but is not a necessity. Together with the strong impact of $\mathrm{Li}^{+}$and $\mathrm{Mg}^{2+}$ ions, this demonstrates that nonelectrostatic effects play an important role for the observed wettability alteration.

Parts of this chapter appear as:

1. B. Bera, N. Kumar, M.H.G. Duits, M.A. Cohen-Stuart, H.T.M. van den Ende \& F. Mugele. Cationic Hofmeister series of mica wettability in alkanes. Manuscript in preparation, (2016). 


\subsection{Introduction}

The importance of a specific type of ion in various ion-surface interactions has been thoroughly investigated since the famous work of Hofmeister [1] about egg-white precipitation in the presence of different cations or anions. From around the same time, studies on the stability of hydrophobic colloids based on the specific influence of an electrolyte have been carried out, with researchers observing the same sequence (known as the lyotropic series) [2]. Almost 130 years have passed since the first report of the phenomenon $[3,4,5,6]$, but there is no universal agreement about the fundamental process governing this ion specificity $[7,8,9,10]$. The wide range of systems where specific ion-adsorption plays a role (starting from macroscopic surface tension effects and osmotic coefficients to microscopic coagulation [11]) has led to many attempts to quantitatively describe the underlying mechanism. In general, the affinity of water to the ions (i.e., hydration) based on these ions' size and charge is acknowledged as the driving factor. Various authors attempted to attribute the complex microscopic interactions to specific mechanisms such as dispersion forces [12], solvation effects [13] or dielectric saturation [14]. Most studies agree that hydration forces control this ion-specific adsorption, but then again, the hydration force is itself a subject of great debate [15]. In this context, the competitive role of electrostatic and chemical forces on the Hofmeister series has also been looked into $[9,16]$. In various recent experimental studies, the hitherto unknown lateral interaction effects between adsorbed monovalent alkali metal $\left(\mathrm{Rb}^{+}\right.$or $\left.\mathrm{Cs}^{+}\right)$ions on mineral surfaces have been demonstrated $[17,18,19]$. From a theoretical perspective, Schwierz et al have looked into the effect of cationic [20] as well as anionic [21] adsorption along the Hofmeister series and the influence of hydrophilic as well as hydrophobic surfaces on surface potentials in great detail using Molecular Dynamics (MD) simulations. They have also studied the same influence for two interfaces, modelling the interaction between the plates using the Poisson Boltzmann (PB) approach [22]. Adsorption and wetting are closely related, as we have discussed in Chapter 2 and Chapter 4. But ion specific effects have not yet been addressed in the existing literature from a wetting perspective. Despite the substantial volume of work on Hofmeister series influenced adsorption properties, there is no example of investigation of wetting properties in a colloidal system based on specific ion-adsorption.

Competitive wetting and wettability alteration are crucial phenomena in 
a host of modern applications such as oil-water separation, sedimentation effects [23, 24], oil recovery etc., e.g., one of the most discussed mechanisms of enhanced oil recovery (EOR) $[25,26,27,28,29,30,31]$ is the oil-wet mineral of reservoir-rock turning into a water-wet surface. Such wettability alteration is a classic wetting phase transition problem for a physical chemist $[32,33,34]$. However, the majority of wetting transition investigations have been done with noble gases and alkanes $[35,36]$ in the vicinity of the bulk critical temperature[37], where no electrolyte is present in the liquid/gas phase. Applications such as EOR, however, rely on the ion-content of an externally introduced phase (waterflooding or surfactant flooding) for wettability alteration since changing the pressure inside the reservoir is not possible. Recent studies have underlined the importance of ion-adsorption from an electrolyte and the subsequent changes in surface charge upon wetting transition $[38,39]$. The influence of the charge of the substrate on contact angle is also well-known through electrowetting [40, 41,5]. Hence it is relevant and interesting to investigate the influence of ion-specific adsorption in a solid/liquid/liquid system and the subsequent effects on wetting and wettability alteration.

In this chapter, we experimentally investigate the effect of ion-specific interactions with the surface (mica) upon the wettability to water in ambient alkane. In Chapter 4 [42], we reported a wettability alteration on a mica surface in ambient oil (decane), when a divalent cation $\left(\mathrm{Ca}^{2+}\right)$ in the salt solution is used instead of a monovalent one. Our results demonstrated the effect of cation-valency on adsorption and wettability for this particular system. We proposed an ion-adsorption based mechanism in order to investigate surface charge reversal caused by divalent cations on the mineral surface. Using the PB approach, we estimated interface potential $\Phi$ in this film based on the surface charge information. In the following work, we vary the types of cations in the salt (with two anions), their concentrations as well as $\mathrm{pH}$ in order to identify a wettability change irrespective of a change in the cation-valency. Subsequently, we discuss and rationalize the experimental results from a specific adsorption point of view. While most of the Hofmeister literature talks about monovalent alkali metal adsorption, it is acknowledged that the binding of divalent cations deserves more 'systematic exploration' [9]. We attempt that as well by looking at the wettability alteration in our system for divalent earth alkali cations. 


\subsection{Experimental Methods}

\subsubsection{Materials}

Anhydrous alkanes (heptane, decane, hexadecane:>99\%, Sigma Aldrich) are the oil phases in the experiments. The oil is passed five times through a vertical column of Alumina powder $\left(\mathrm{Al}_{2} \mathrm{O}_{3}\right.$, Sigma Aldrich, Puriss grade $\left.>98 \%\right)$ to remove surface-active impurities until the interfacial tension (measured using Pendant Drop Method at the alkane-water interface) is constant within $2 \mathrm{mN} / \mathrm{m}$ for at least $20 \mathrm{~min}$. Ultrapure water (Millipore, Synergy UV Instruments, resistivity $18 \mathrm{M} \Omega$ ) is used for preparing salt solutions. We prepared all inorganic salt (Sigma Aldrich) solutions in a concentration range of $1 \mathrm{mM}$ to $1 \mathrm{M}$ by adding the ultrapure water to a carefully measured amount of the required salt. The $\mathrm{pH}$ of the solutions is adjusted between 2 and 10 using $\mathrm{HCl} / \mathrm{HNO}_{3}$ and $\mathrm{NaOH}(0.1 \mathrm{M}$, Sigma Aldrich). Muscovite mica (B\&M Mica Company Inc., USA; initial thickness $340 \mu \mathrm{m}$ ) and oxidized silicon wafers with an amorphous silicon oxide layer (thickness: $30 \mathrm{~nm}$ ) mimicking silica are the solid substrates in the experiments. Mica sheets are cleaved inside the oil using a needle and a tweezer to obtain a pristine surface during the experiment. We have also tested a parallel protocol where the mica is cleaved in air with scotch tape and then immersed in oil. The latter protocol does not result in any significant change in the experimental results. Silica surfaces are cleaned using a combination of Piranha solution (mixture of $30 \%$ conc. $\mathrm{H}_{2} \mathrm{O}_{2}$ and $70 \%$ conc. $\mathrm{H}_{2} \mathrm{SO}_{4}$ ) followed by extensive rinsing with ultrapure water and plasma treatment for $30 \mathrm{~min}$.

\subsubsection{Optical Goniometry}

The wetting of aqueous drops on mica/silica is characterized by measuring static contact angles, using a commercial contact angle goniometer (OCA 20L, Dataphysics Instruments GmBH, Germany). The measurement is based on sessile-drop method using aqueous drops of volume $2 \mu \mathrm{l}$ placed on the substrate. The accuracy in the drop-volume is measured to be within \pm 0.2 $\mu \mathrm{l}$. All experimental datapoints are average of 25 independent measurements (drops) placed on at least 5 different substrates. Before placing the aqueous drops on the substrates, pendant drop measurements are performed to determine the oil/water interfacial tension (IFT).

The contact angle of the drop is extracted from video snapshots using the tangent and circle-fitting data analysis software (SCA 22) provided with the 
instrument. Contact angles can be determined with a relative accuracy of $\pm 1^{\circ}$. The minimum contact angle that can be determined on reflective surfaces is approximately $2^{\circ}$, because of the fundamental limitations of optical imaging. Normally, contact angles in the range of a few degrees are quite challenging to measure using goniometry method. But we have standardized the imaging and illumination conditions to compare the data for different salts, and we can measure the relative trends in these small contact angles. It was not possible to perform advancing-receding measurements, since the receding contact angle for most of these small values is $0^{\circ}$. In addition, we measure the base diameter of our drops optically, and fit the contact angles with a spherical cap approximatrion for better accuracy. Various technical aspects of our goniometry measurements are discussed in detail in Chapter 3.

\subsubsection{Atomic Force Microscopy}

An atomic force microscope (Cypher S, Asylum Research, CA, USA) is used to investigate the adsorption of various species on the mineral surface. These experiments are performed ex-situ, with a silica tip on mica surface. The AFM is operated in non-contact mode (amplitude modulation) for obtaining atomic resolution at the mineral adsorption sites. The stiffness of the cantilever is measured $\left(\mathrm{k}_{\text {stiff }}\right)$, and with the information of the tip-geometry, the force-curves are obtained. Reference measurements are done with silica tip-silica substrate interactions, and the interaction is substracted from the mica-silica tip interaction before fitting the result to a charge-regulation model for obtaining surface charge at mica-water interface.

\subsection{Results}

\subsubsection{Order of Monovalent Cations}

Figure 6.1 shows the contact angles for chloride salts of monovalent alkali ions on mica in ambient decane at various concentrations $(1,10,100$ and 1000 $\mathrm{mM}$ ) for $\mathrm{pH}$ values of 3,6 , and 9 . For $\mathrm{NaCl}$ and $\mathrm{KCl}$, the contact angle is $<2^{\circ}$ for all $\mathrm{pH}$ 's, as reported in Chapter 4 . Since these extremely low values fall below our detection limit, we represent them as ' 0 in Figure 6.1 (dark cyan diamonds connected by dotted lines). Finite contact angles (which can be measured) are noticed for $\mathrm{LiCl}$ at $1 \mathrm{M}$ concentration, where a very small contact angle $\sim 2.5^{\circ}$ is observed. Subsequently, we notice low 


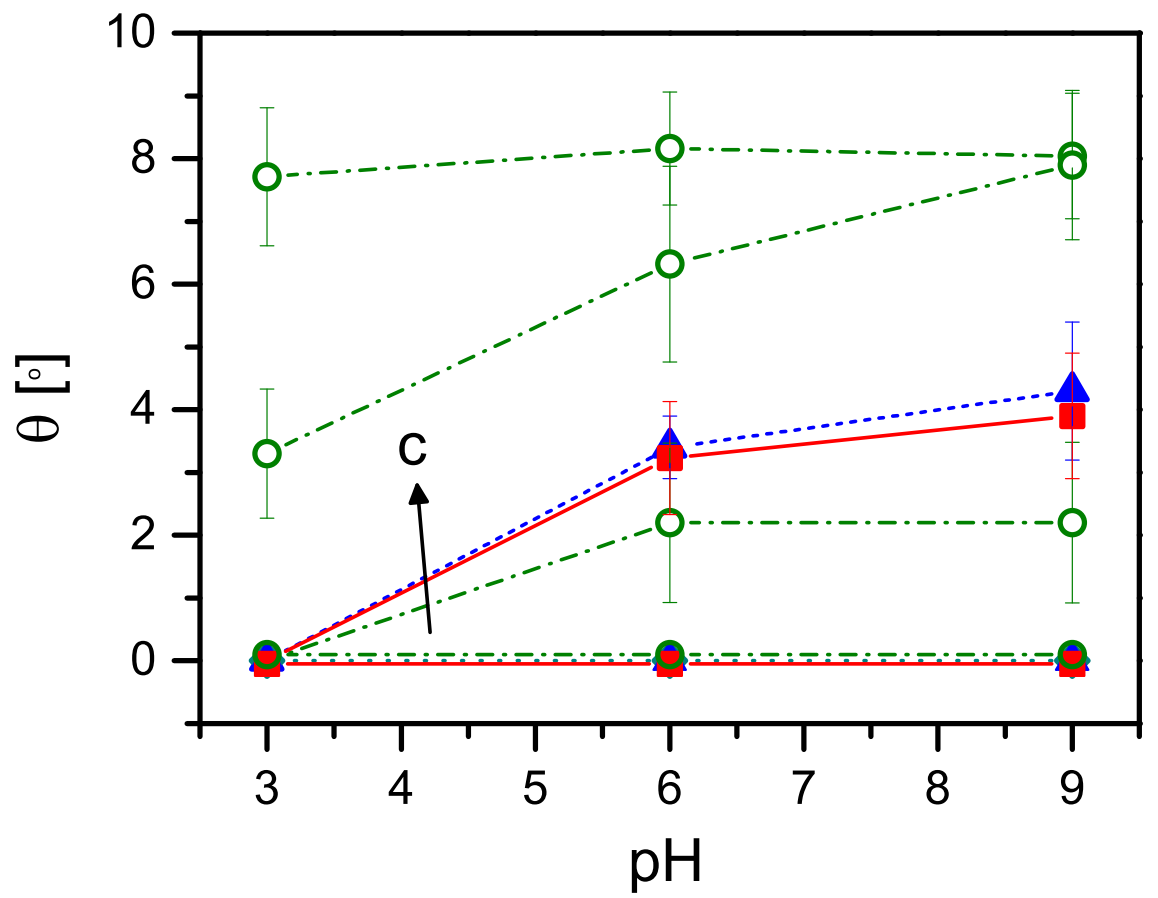

Figure 6.1: Equilibrium contact angle of aqueous drops on mica in ambient decane vs the $\mathrm{pH}$ of the salt soution in the drop for various monovalent salts: $\mathrm{NaCl} / \mathrm{KCl}$ (dark cyan diamonds, dotted lines), $\mathrm{LiCl}$ (red squares, solid lines), $\mathrm{RbCl}$ (blue triangles, dashed lines) \& $\mathrm{CsCl}$ (open green circles, dash-dot lines). The symbols are experimental datapoints (average of 25 independent experiments, standard deviation shown). All contact angles $\theta<2^{\circ}$ are not measurable and assigned a value of $0^{\circ}$. The arrow with the letter ' $c$ ' represents direction of increasing concentration among $1 \mathrm{mM}, 10 \mathrm{mM}, 100 \mathrm{mM}$ and $1 \mathrm{M}$.

but distinguishable contact angles ( 2.5-3.5 , on mica in decane) for $\mathrm{RbCl}$, at a concentration of $1 \mathrm{M}$. For all other lower concentrations of $\mathrm{LiCl}$ and $\mathrm{RbCl}$ in the aqueous phase, the drops have contact angle $\theta<2^{\circ}$ (Figure 6.1). $\mathrm{CsCl}$ solution, on the other hand, already shows a measurable finite contact angle on mica in decane at $10 \mathrm{mM}$ concentration, and as we increase the salt concentration, the contact angles increase as well, with $\theta$ as high as $7-8^{\circ}$ at 1 $\mathrm{M} \mathrm{CsCl}$ concentration (Figure 6.1). 


\subsubsection{Order of Divalent Cations}

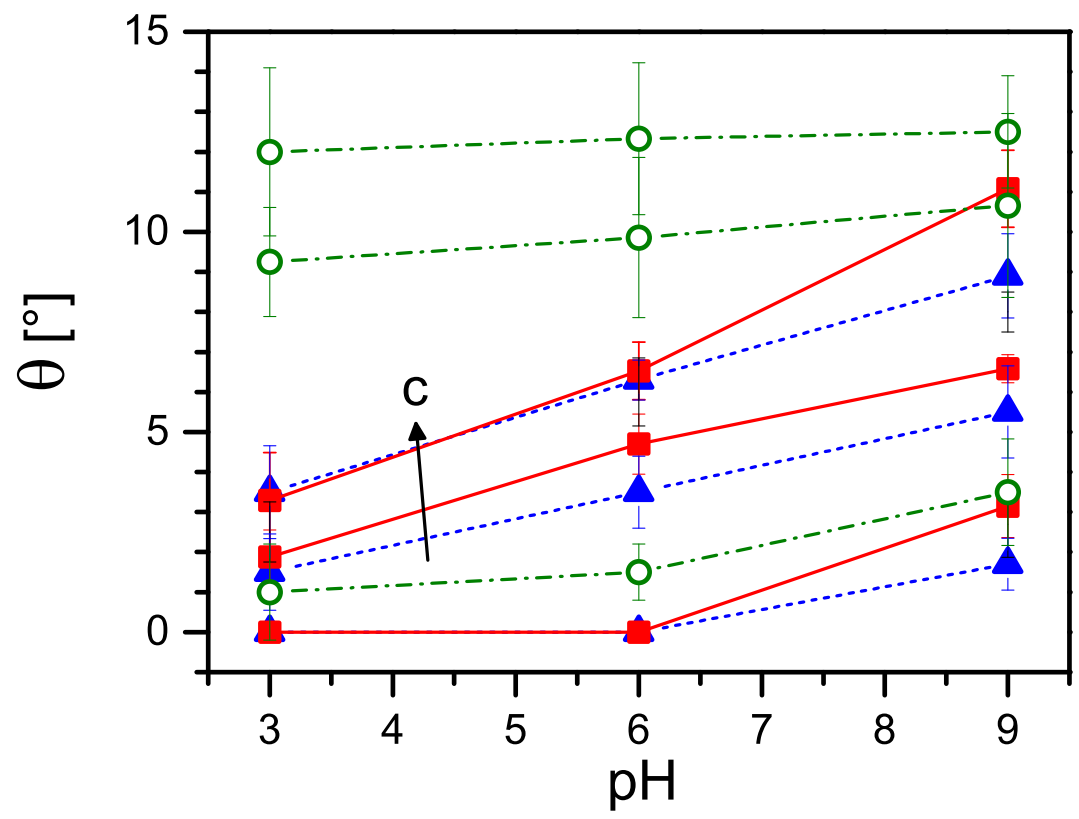

Figure 6.2: Equilibrium contact angle of aqueous drops on mica in ambient decane vs the $\mathrm{pH}$ of the salt soution in the drop for various divalent salts: $\mathrm{CaCl}_{2}$ (blue triangles, dashed lines), $\mathrm{MgCl}_{2}$ (red squares, solid lines) \& $\mathrm{BaCl}_{2}$ (open green circles, dash-dot lines). The symbols are experimental datapoints (average of 25 independent experiments, standard deviation shown). All contact angles $\theta<2^{\circ}$ are not measurable and assigned a value of $0^{\circ}$. The arrow with the letter ' $c$ ' represents direction of increasing concentration among $1 \mathrm{mM}, 10$ $\mathrm{mM}, 100 \mathrm{mM}$ and $1 \mathrm{M}$.

Figure 6.2 shows the contact angles for divalent salts wetting mica in decane at various salt concentrations $(1,10,100$ and $1000 \mathrm{mM})$. Above a threshold concentration which depends on the type of salt and above $\mathrm{pH} 4$, the aqueous phase with a divalent cationic salt always gives $\theta>2^{\circ}$ on mica in ambient oil. For $\mathrm{MgCl}_{2}$, this threshold concentration is about $50 \mathrm{mM}$, where we see a drop of finite contact angle (Figure 6.2). For $\mathrm{CaCl}_{2}$ as well, the threshold concentration for a macroscopic finite contact angle is around $\sim$ $50 \mathrm{mM}$, though the corresponding contact angles for a $\mathrm{CaCl}_{2}$ drop seems to be slightly smaller than that of $\mathrm{MgCl}_{2}$. Our investigation of another divalent 
salt, Barium Chloride $\left(\mathrm{BaCl}_{2}\right)$, already produces measurable contact angles at a lower concentration ( $\sim 10 \mathrm{mM}$ concentration). We notice in Figure 6.2 that the values of $\theta$ first decrease as we go from $\mathrm{MgCl}_{2}$ to $\mathrm{CaCl}_{2}$, but increase again for $\mathrm{BaCl}_{2}$. The effect of $\mathrm{pH}$-change on the contact angles for $\mathrm{BaCl}_{2}$ are less compared to other divalent salts, i.e., unlike $\mathrm{CaCl}_{2}$ and $\mathrm{MgCl}_{2}$, if we increase the $\mathrm{pH}$ from 3 to 6 and 9, there is no significant change in $\theta$ for a drop of $\mathrm{BaCl}_{2}$ solution

Figure 6.3 shows the gradual increase for various cationic salt solutions (both monovalent and divalent) at $1 \mathrm{M}$ salt concentration and $\mathrm{pH} 9$. Based on the trend, we propose the existence of a unique cationic series of mica wettability in ambient oil, which is comparable to a classic Hofmeister series of protein precipitation. We call this series 'Cationic Hofmeister series of wettability':

$$
\mathrm{Na}^{+}, \mathrm{K}^{+}<\mathrm{Li}^{+}<R b^{+}<\mathrm{Cs}^{+}<\mathrm{Ca}^{2+}<\mathrm{Mg}^{2+}<\mathrm{Ba}^{2+}
$$
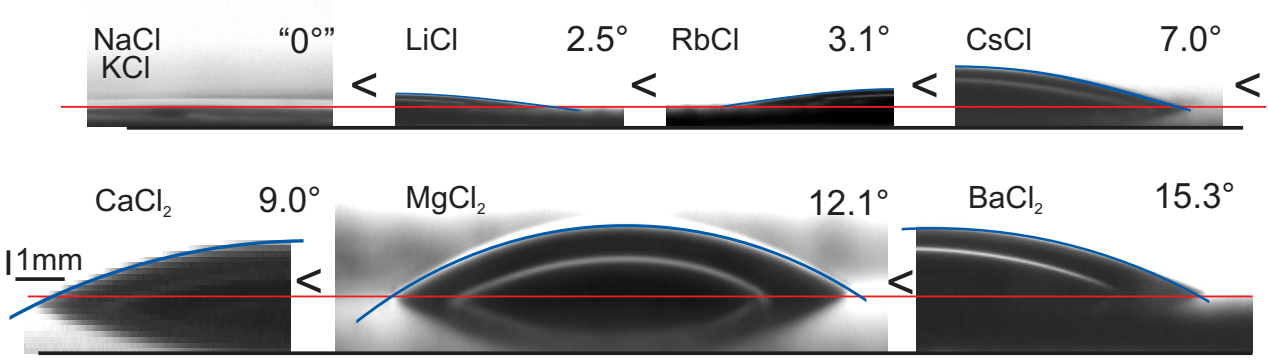

Figure 6.3: Cationic Hofmeister series of wettability alteration on mica in ambient decane with aqueous drop of various salt solutions; The difference in the wetting trend between various monovalent and divalent salt solutions is clear from the snapshots of drops (of aqueous salt solution, $1 \mathrm{M}$ salt concentration, $\mathrm{pH} 9$ ) on mica in decane: a $\mathrm{NaCl} / \mathrm{KCl}$ drop has $\sim 0^{\circ}$ contact angle on mica, almost completely wetting the substrate), For $\mathrm{RbCl}$ there is a very small but distinguishable finite contact angle; With $\mathrm{CsCl}$, the contact angle is $\sim 7^{\circ}$; in comparison, $\mathrm{MgCl}_{2}, \mathrm{CaCl}_{2}$ and $\mathrm{BaCl}_{2}$ drops all have finite contact angle on mica, indicating partial wetting of various degrees. For all the drops, the blue lines indicate the circle fitting the drop-file which eventually provides the contact angle. 

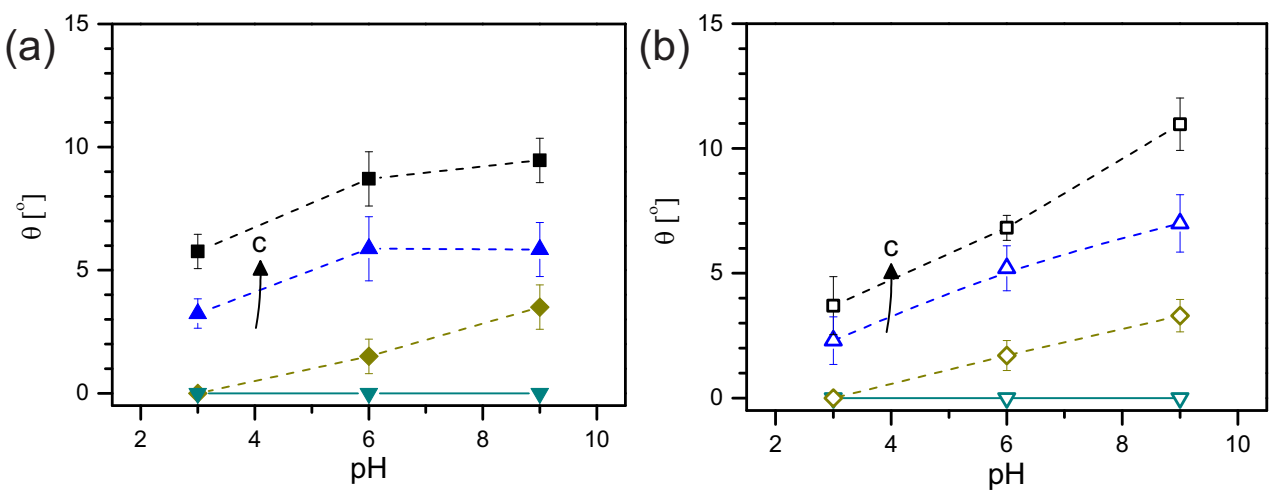

Figure 6.4: Equilibrium contact angle on mica in decane for nitrate salts (a) $\mathrm{Ca}\left(\mathrm{NO}_{3}\right)_{2}$ (closed symbols) and (b) $\mathrm{Mg}\left(\mathrm{NO}_{3}\right)_{2}$ (open symbols), vs $\mathrm{pH}$ of aqueous solution at various salt concentration:1 $\mathrm{M}$ (black squares), $100 \mathrm{mM}$ (blue upward triangles), $50 \mathrm{mM}$ (olive diamonds) and $<10 \mathrm{mM}$ (dark cyan downward triangles). Each datapoint is an average of 25 individual drops under identical conditions with the standard error of the measurements shown. The straight lines are guides to the eye. The arrow with the letter ' $c$ ' represents direction of increasing concentration.
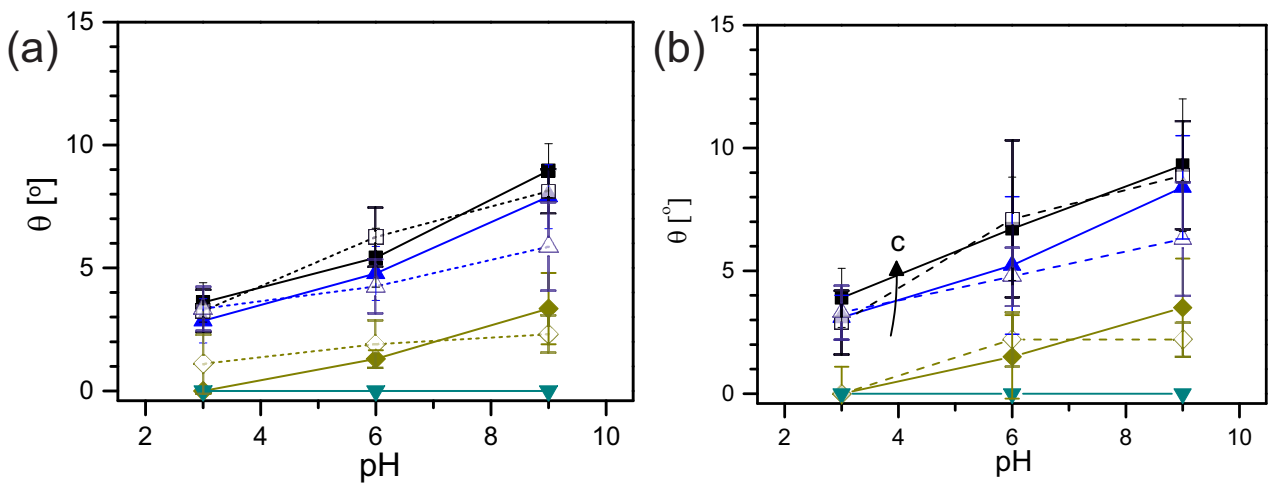

Figure 6.5: Equilibrium contact angle on mica in (a) heptane and (b) hexadecane, vs $\mathrm{pH}$ of aqueous solution with $\mathrm{CaCl}_{2}$ (full symbols) and $\mathrm{Ca}\left(\mathrm{NO}_{3}\right)_{2}$ (open symbols) at various salt concentration: $1 \mathrm{M}$ (black squares), $100 \mathrm{mM}$ (blue upward triangles), $50 \mathrm{mM}$ (olive pentagons) and $<10 \mathrm{mM}$ (dark cyan downward triangles). Each datapoint is an average of 25 individual drops under identical conditions with the standard error of the measurements shown. The straight lines are guides to the eye. The arrow with the letter ' $c$ ' represents direction of increasing concentration. 


\subsubsection{Influence of Anion, Alkane and $\mathrm{pH}$}

We have extended the wetting experiments to salts with the $\mathrm{NO}_{3}^{-}$anion as well as to ambient alkanes of different aliphatic chain length. Figure 6.4 shows the contact angles on mica in decane for (a) $\mathrm{Ca}\left(\mathrm{NO}_{3}\right)_{2}$ and (b) $\mathrm{Mg}\left(\mathrm{NO}_{3}\right)_{2}$. It is clear that there is no significant change in the contact angle based on the type of anion. Figure 6.5 depicts the contact angles on mica for $\mathrm{CaCl}_{2}, \mathrm{Ca}\left(\mathrm{NO}_{3}\right)_{2}, \mathrm{MgCl}_{2}$ and $\mathrm{Mg}\left(\mathrm{NO}_{3}\right)_{2}$ in ambient heptane (a) and hexadecane (b). The contact angle trends and values are closely comparable to those in decane. We have also investigated the wetting for $\mathrm{CaCl}_{2}$ and $\mathrm{MgCl}_{2}$ salt solutions for the entire range of (integer) $\mathrm{pH}$ values between $\mathrm{pH} 3$ and $\mathrm{pH} 10$ (Figure 6.6). They also follow the trend already observed at $\mathrm{pH} 3,6$ and 9.

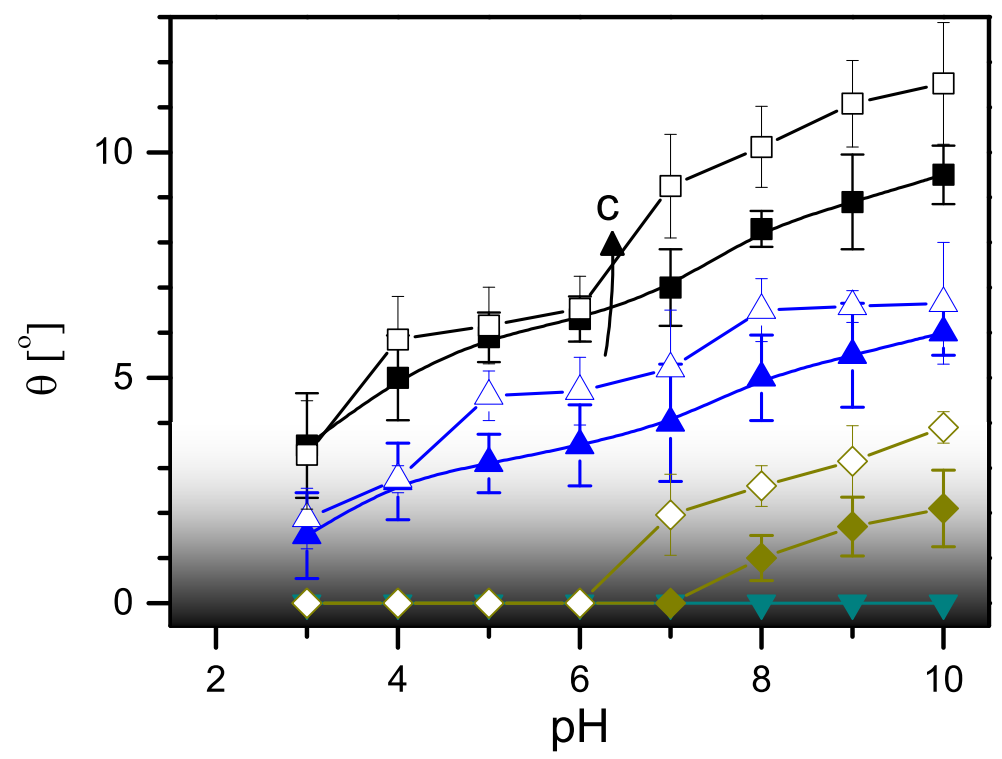

Figure 6.6: Equilibrium contact angle on mica in decane vs $\mathrm{pH}$ of aqueous solution with $\mathrm{CaCl}_{2}$ (full symbols) and $\mathrm{MgCl}_{2}$ (open symbols) at various salt concentration: $1 \mathrm{M}$ (black squares), 100mM (blue upward triangles), $50 \mathrm{mM}$ (olive pentagons) and $<10 \mathrm{mM}$ (dark cyan downward triangles). Each datapoint is an average of 25 individual drops under identical conditions with the standard error of the measurements shown. The straight lines are guides to the eye. The arrow with the letter ' $c$ ' represents direction of increasing concentration. 
The results discussed so far, clearly imply that the phenomenon of wetting alteration on the mineral surface is not exclusively limited to a change of cation valency, but it depends on which specific cation is being introduced in the system. We observe a Hofmeister-like sequence in the wettability of aqueous electrolytes in an oil medium. In the following section, we revisit the ion-adsorption based argument for wetting alteration from Chapter 4 and propose modifications in the mechanism in order to explain the various observations with ion-specific wettability.

\subsection{Mechanistic Insight}

A change in contact angle can be attributed to a change in the corresponding interface energies (Figure 6.7) i.e., the surface tensions of the various phases involved. Young's equation [32] is written as:

$$
\cos \theta=\frac{\gamma_{s o}-\gamma_{s w}}{\gamma_{o w}}
$$

where, $\theta$ is the contact angle and $\gamma_{s o}, \gamma_{s w}$ and $\gamma_{o w}$ are the interfacial ten-

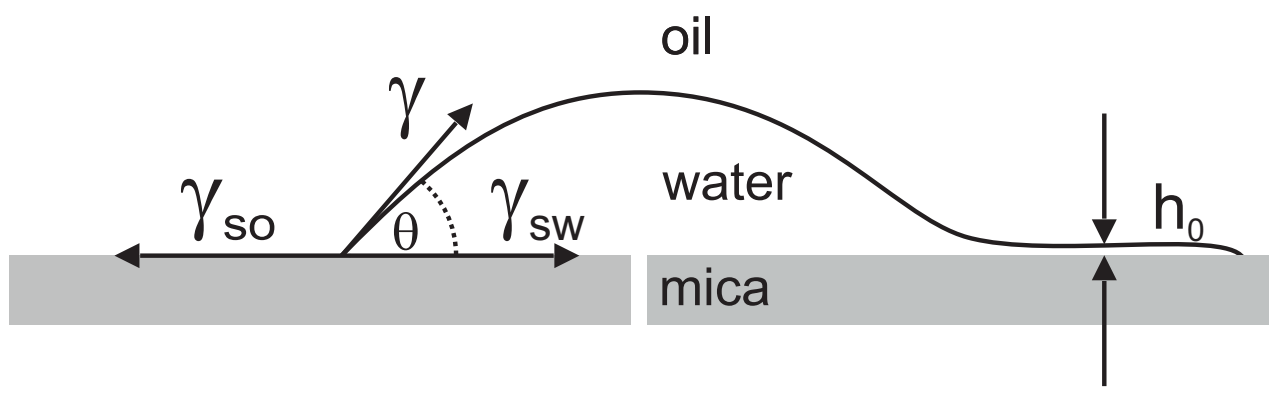

Figure 6.7: Schematic of a partial wetting situation on mica in ambient decane; The force balance arising from interfacial tensions resulting in a finite contact angle $\theta$ (left), while such a finite contact angle implies a molecularly thin aqueous film co-existing with the drop (right). Figure 4.3 is redrawn here.

sions at mineral-oil, mineral-water and oil-water interfaces, respectively. As salt concentration is increased, there is more depletion of cations from a hydrophobic interface (in this case, the alkane-water interface) leading to an increase in the IFT. We have carried out pendant drop measurements of interfacial tension at the oil/water interface and a small increase at the oil/water interfacial tension is observed, which corroborate previous studies (Figure 
$6.8,[43,44])$. However, it is well known, that if we follow the increasing order of cation-size, e.g., for monovalent cations, $\mathrm{Li}^{+}<\mathrm{Na}^{+}<\mathrm{K}^{+}<R b^{+}<$ $\mathrm{Cs}^{+}$, then the increment in interfacial tension at the oil-water interface $\left(\gamma_{s o}\right)$ decreases [45]. This implies that if $\gamma_{s o}$ and $\gamma_{s w}$ are constant, then following the decrease in $\gamma_{o w}$, the contact angle should decrease as the cation-size in the electrolyte increases, which is contrary to our experimental observation. Furthermore, $\gamma_{s w}$ or $\gamma_{s o}$ do not remain constant as well; e.g., an increase in salt concentration normally results in a decrease of $\gamma_{s w}$ [46], since the formation of a double layer is more spontaneous with more ions present in the aqueous phase. Hence it is advantageous to focus upon the microscopic picture of wetting in order to understand the proceedings in our three-phase wetting situation.

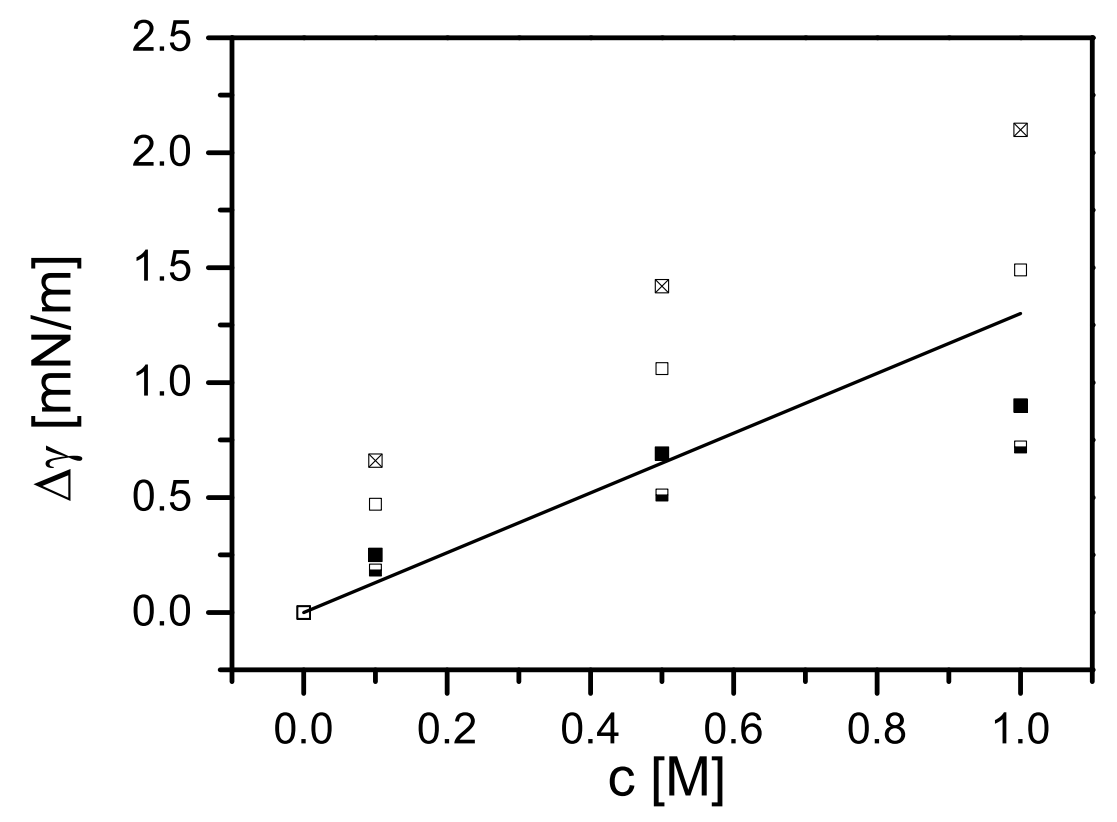

Figure 6.8: Interfacial tension (IFT) at decane/water interface with respect to salt concentration in the aqueous phase for $\mathrm{NaCl}$ (closed squares), $\mathrm{KCl}$ (halfclosed squares), $\mathrm{CaCl}_{2}$ (crossed squares) and $\mathrm{MgCl}_{2}$ (open squares). The symbols are experimental datapoints. Decane/ $\mathrm{NaCl}$ IFT is compared with previous studies, denoted by the straight line [45].

Wetting of a solid surface is effectively the formation of a thin layer 
of the wetting phase on the substrate, as shown in Figure 6.7, right. For such a thin aqueous film of equilibrium thickness $\mathrm{h}_{0}, \gamma_{s o}$ can be written as $\left(\gamma_{s w}+\gamma_{o w}+\Phi\left(h_{0}\right)\right.$, where $\left.\Phi\left(h_{0}\right)\right)$ is the interaction potential in the thin aqueous film. Changes in $\Phi\left(h_{0}\right)$ are governed by the adsorption of counter-ions from this thin film to the interfaces. In presence of a monovalent salt solution (schematic shown in Figure 6.9c, above left), the counter-ion adsorption at the mineral interface is not strong enough to cause overcharging of the usually negatively charged mica-water interface. The oil-water interface remains negatively charged under all salt concentrations $(>\mathrm{pH} 4)$, since the mechanism of charging at a hydrophobic interface does not depend on cation adsorption [47, 48]. As a result, the mica-water and the oil-water interfaces have a repulsive interaction between them, or, in other words, the disjoining pressure in the thin aqueous film is positive, making the film inifintely thick (compared to molecular length-scale, $\sim 10 \mathrm{~nm}$ film is considered infinite) and creating a macroscopically indistinguishable contact angle. Divalent cations, on the other hand, have two units of charge per cation and when they adsorb to a mineral surface like mica, it causes overcharging above a threshold salt concentration. E.g., charge reversal has been reported for $\mathrm{CaCl}_{2}$ above $\sim 30 \mathrm{mM}$ salt concentration [49]. Figure 6.9c, left below, depicts the schematic representation of the divalent situation. The oppositely charged mica-water and oil-water interfaces have an attractive interaction (or a negative disjoining pressure) between them, creating a molecularly thin aqueous film along with a co-existing aqueous drop of macroscopically measurable contact angle.

\subsubsection{Ion-specific Adsorption}

However, based on the experimental observations described in this chapter, the cation wettability series on mica from our experiments is clearly not solely driven by electrostatics. We observe a partial wetting situation on mica for monovalent cations such as $\mathrm{Li}^{+}, \mathrm{Rb}^{+}$or $\mathrm{Cs}^{+}$, where we can expect that the surface charge is not reversed. Ion size, hydration, hydrated surface etc. contribute significantly to our picture of contact angle change. Following the Hofmeister series, the adsorption of metal ions on a sufficiently negative mineral surface increases as the ion-size increases. So, the adsorption on mica should follow the order: $N a^{+}, K^{+}<R b^{+}<C s^{+}$, which is exactly same as the order of increasing contact angle we found in the experiments. In Figure 6.9c, above right, we have sketched a wetting situation with con- 


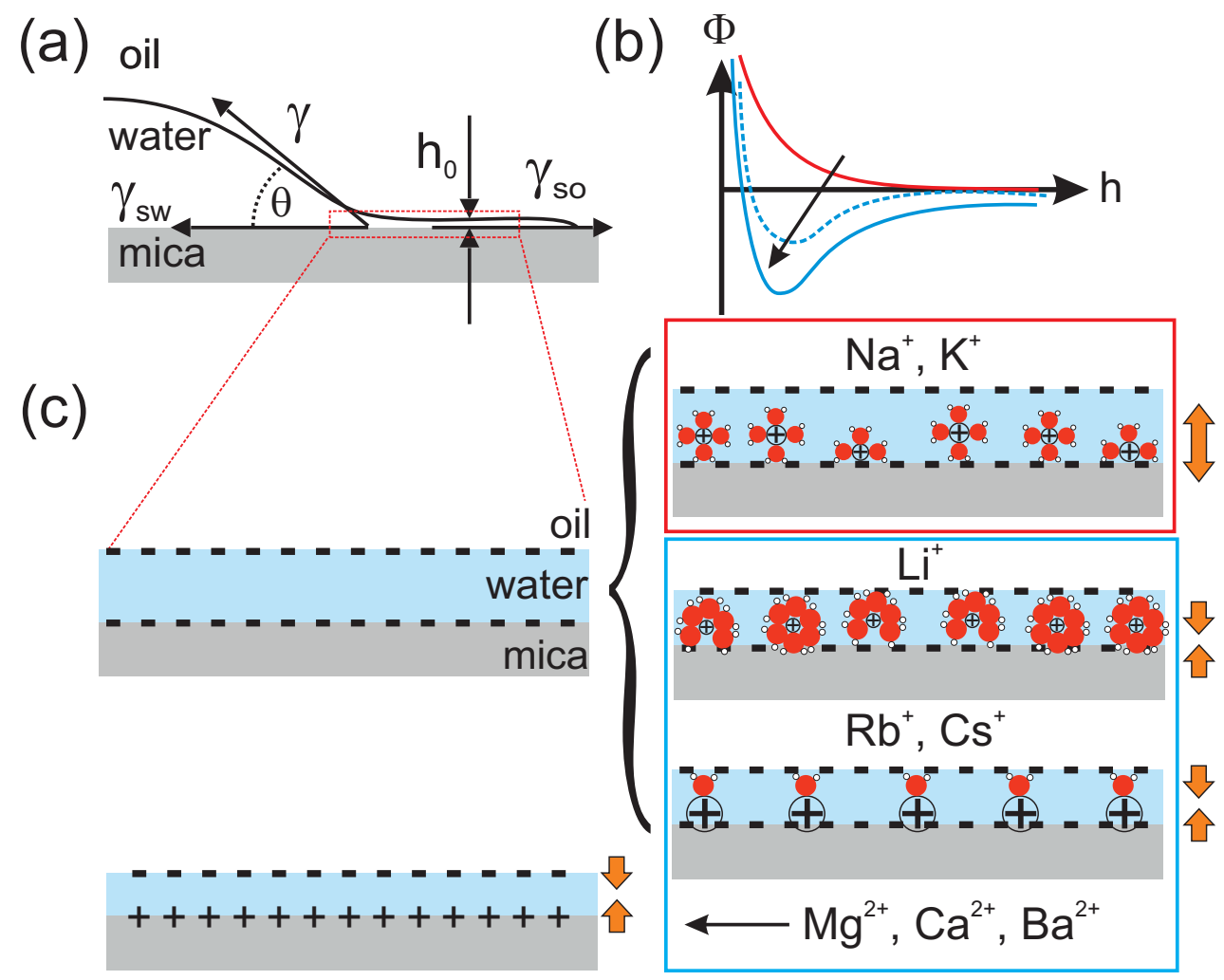

Figure 6.9: (a) Force balance arising from interfacial tensions leading to the contact angle $\theta$ of a drop requires the supplementary contribution of interaction potential $\Phi(h)$ when there is a molecularly thin film of equilibrium thickness $h_{0}$ present next to the drop (b) Schematic examples of repulsive and attractive $\Phi(h)$, where negative $\Phi(h)$ with a pronounced minimum leads to a finite contact angle. The arrow depicts the gradual change of cations following our 'cationic Hofmeister series'. (c) Left: in the case of monovalent cations, both mica-water and oil-water interfaces remain negatively charged (above), while divalent cations above a certain concentration reverses the surface charge of mica (below). Right: Ion specific adsorption leads to different results even among monovalent cations; a moderate sized and moderately hydrated (water molecule represented by red oxygen atom and white hydrogen atoms) ion: $\mathrm{Na}^{+}$or $\mathrm{K}^{+}$(above) adsorb less strongly. Very small cations (middle) such as $\mathrm{Li}^{+}$get hydrated strongly and as a result adsorb significantly with their large hydrated size. Larger (bare) cations such as $\mathrm{Cs}^{+}$(bottom) adsorb strongly due to their large size despite lack of hydration; the large size leads to lateral interaction as well as interaction with the oil-water interface. 
tact angle $\theta<2^{\circ}$ for monovalent cations such as $\mathrm{Na}^{+}, \mathrm{K}^{+}$, represented by the + ions. The mica surface is negatively charged, and although there is cation adsorption at mica interface, it is expected to not be sufficient for a charge-reversal. The cations are hydrated as well (represented by the water molecule around the + sign), but even the hydrated cations are far enough from the oil-water interface for strong interaction. As a result the interaction between mica-water and oil-water interface remains repulsive, and there is no perceivable contact angle.

Figure $6.9 \mathrm{c}$, middle right, portrays the scenario for adsorption of monovalent ions with a large hydrated ion radius, such as $\mathrm{Li}^{+}$. It has the smallest bare ion-radius among the cations under investigation and therefore, should refrain from adsorbing strongly on a negatively charged mica surface. However, being the smallest cation, $\mathrm{Li}^{+}$is hydrated strongly and has the largest hydrated ion radius [50] (Table 6.1). As a result, during adsorption on mica, $\mathrm{Li}^{+}$is effectively larger in comparison to $\mathrm{Na}^{+}$or $\mathrm{K}^{+}$and adsorbs more strongly. Furthermore, the size of the hydrated cation could be of the order of the equilibrium film thickness, as depicted in the figure. In this case, the oil-water interface might interact directly with the hydrated cation which influences the total interaction between two interfaces.

Figure 6.9c, bottom right, depicts the situation for adsorption of monovalent ions with a larger bare ion radius, represented by a bigger + symbol. As the mica surface charge is gradually nullified by the adsorption of relatively bigger monovalent cations, $\mathrm{Cs}^{+}$for example, there is a reduced electrostatic interaction between the two interfaces. At the same time, these adsorbed species have more freedom to interact with each other and previous studies have reported a lateral interaction between these ions [17], forming an organized layer next to the mineral surface and screening the negatively charged surface.

The mechanism of cationic wettability ordering is almost identical for various divalent cations, i.e., $\mathrm{Mg}^{2+}, \mathrm{Ca}^{2+}$ and $\mathrm{Ba}^{2+}$. As shown in Figure 6.9c, bottom left, divalent cations result in a charge reversal on mica surface above a threshold concentration and $\mathrm{pH}$. This leads to an attractive interaction between the mica-water and oil-water interfaces, leading to a finite contact angle. However, the relative increase of $\theta$ from one divalent cation to other is still a result of ion-specific adsorption. $\mathrm{Mg}^{2+}$ is the smallest bare cation among our experimental systems, but in hydrated state the cation is 


\begin{tabular}{|c|c|c|}
\hline Ion & $\begin{array}{c}\text { Bare Ion } \\
\text { Radius [nm] }\end{array}$ & $\begin{array}{c}\text { Hydrated Ion } \\
\text { Radius [nm] }\end{array}$ \\
\hline $\mathrm{Li}^{+}$ & 0.094 & 0.382 \\
$\mathrm{Na}^{+}$ & 0.117 & 0.358 \\
$\mathrm{~K}^{+}$ & 0.149 & 0.331 \\
$\mathrm{Rb}^{+}$ & 0.163 & 0.329 \\
$\mathrm{Cs}^{+}$ & 0.186 & 0.329 \\
$\mathrm{Mg}^{2+}$ & 0.072 & 0.428 \\
$\mathrm{Ca}^{2+}$ & 0.100 & 0.412 \\
\hline
\end{tabular}

Table 6.1: Bare ion radii and hydrated ion radii for various cations used in wetting experiments. Table reproduced from [50].

larger and gets adsorbed more strongly than $\mathrm{Ca}^{2+}$ resulting in a higher positive charge on mica. $\mathrm{Ba}^{2+}$, on the other hand, is devoid of a hydration shell but is the largest bare cation among these three and hence adsorbs most strongly. This also implies a higher positive charge on mica surface compared to $\mathrm{Mg}^{2+}$ and $\mathrm{Ca}^{2+}$.

These various adsorption situations have a pronounced effect upon the interaction potential $\Phi$, as depicted in Figure $6.9 \mathrm{~b}$. The interaction potential for $\mathrm{Na}^{+}, \mathrm{K}^{+}$adsorption is shown by the solid red line, where interaction between negatively charged mica and oil interfaces leads to a repulsive interface potential. For $\mathrm{Li}^{+}, \mathrm{Rb}^{+}$or $\mathrm{Cs}^{+}$, a weakly negatively charged mica interacts with negatively charged oil interface through a closely-knit screening layer of these positively charged ions. This positively charged layer gives rise to an interaction where van der Waals and steric contributions are significant, and hence an interaction with a shallow minimum (denoted by the dashed blue line) originates resulting in a small but finite $\theta$. For divalent cations, the interface potential $\Phi$ depends on the adsorption of the specific cation, and positively charged mica has an attractive interaction with the negatively charged oil interface (solid blue dashed line). The depth of the potential well is a direct indication of the contact angle $\theta$.

\subsubsection{Force Measurements}

Force measurements are reliable in the context of investigating ion-specific adsorption and our AFM experiments have captured the adsorption pic- 
ture at mica-water interface as we follow the cation series in the salt solution (Fig.6.10, [51]). It is observed that, as we go to a bigger monovalent cation (radius of bare cation, in the experiments, $\mathrm{Na}^{+}$and $\mathrm{Cs}^{+}$are compared), the adsorption at mica surface is more pronounced, but there is no charge-reversal. With $\mathrm{Mg}^{2+}$ and $\mathrm{Ca}^{2+}$, however, the surface charge on mica clearly changes sign above $\sim 30 \mathrm{mM}$ concentration. Hence, in the case of a divalent cation, we can definitely support the arguments and modelling presented in Chapter 4 and 5 that the charge of our oil-water interface remains negative under all conditions of our wetting experiments, whereas the mica-water interface switches from negative to positive surface charge at sufficiently high $\mathrm{Ca}^{2+}$ or $\mathrm{Mg}^{2+}$ concentrations and $\mathrm{pH}$, in agreement with the observed wetting transition.

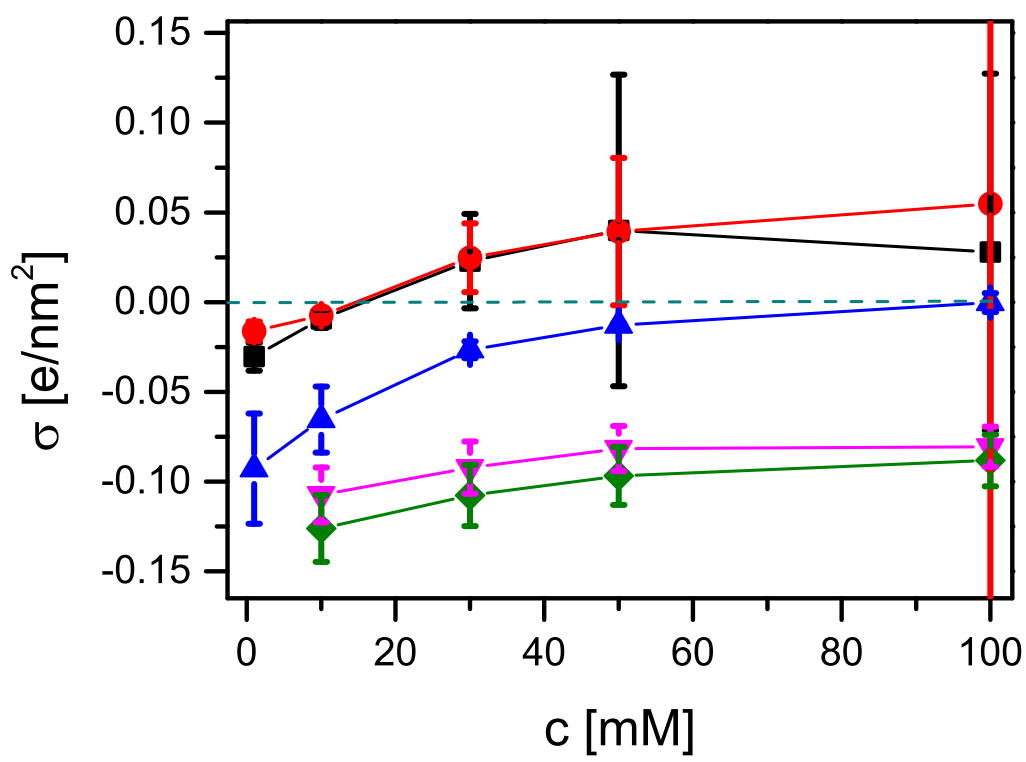

Figure 6.10: Surface Charge $(\sigma)$ at mica interface with aqueous solution of various salts: $\mathrm{LiCl}$ (green diamonds), $\mathrm{NaCl}$ (pink downward triangles), $\mathrm{CsCl}$ (blue triangles), $\mathrm{CaCl}_{2}$ (red circles) and $\mathrm{MgCl}_{2}$ (black squares). The symbols are experimental datapoints based on 3 independent AFM experiments under identical conditions. The straight lines are guide to the eye. Figure reproduced with permission from [51]. 


\subsubsection{Discussion}

Throughout the previous section, various interactions between ions and interfaces as well as between two interfaces have been described in the context of the adsorption mechanism. In the present section, we will discuss the individual interactions as well as their effect on our wettability series. Electrostatic Coulombic interaction is a crucial contribution to our adsorption model, in which the interaction force $\mathrm{F}_{c}$ between two charged object is given by: $F_{c} \propto \frac{q_{1} q_{2}}{r_{12}^{2}}$, where, $\mathrm{q}_{1}$ and $\mathrm{q}_{2}$ are the charges of the species and $\mathrm{r}_{12}$ is the distance between them. In our adsorption model, if the bare ion radius $\left(\mathrm{r}_{0}\right)$ increases, then this interaction becomes weaker, leading to less adsorption. However, one of the primary assertions of the classical Hofmeister series is the 'like seeks like' argument [9], i.e., depending on the specific system, a smaller unhydrated ion prefers a smaller unhydrated adsorption site, while a larger unhydrated ion likes a larger unhydrated adsorption site. In other words, as the cation size increases, we can expect a stronger adsorption on a sufficiently negatively charged surface such as our system. The phenomenon can be attributed to the dispersion forces $\left(\mathrm{F}_{d}\right)$ in action, which can be written as: $F_{d} \propto \frac{\alpha^{2}}{r_{12}^{6}}$, where $\alpha$ is the polarizability of the species and increases as the ion size increases following the relation $\alpha \propto r_{0}^{3}$. The effect of dispersion in adsorption and Hofmeister series has been demonstrated by a body of literature $[11,12]$. The competition of coulombic and dispersion interactions play an important role in the ordering of adsorption.

We have also pointed out that hydration plays a major role in ion-specific adsorption. This is clear from the behavior of cations such as $\mathrm{Li}^{+}$or $\mathrm{Mg}^{2+}$, which are known to be strongly hydrated and adsorb stronger than the bare ion. As discussed in the previous section, hydration of a cation leads to an effective hydrated radius $\left(\mathrm{r}_{H}\right)$, which governs the adsorption onto the solid substrate. Clearly, hydrated ion radius is also not the only governing factor for the amount of adsorption: among monovalent cations, $\mathrm{Li}^{+}$has the largest hydrated ion radius while $\mathrm{Cs}^{+}$the smallest (Table 6.1), which would suggest $\mathrm{Li}^{+}$should be the most strongly adsorbed ion and produce the largest contact angle, which is contrary to our observation. Hence, we can conclude that hydration of ions and surfaces play significant role in specific adsorption mechanism, but due to the competition of coulombic and dispersion forces, a monotonic adsorption behaviour only depending on the hydrated states of the species is absent. 
It is possible to quantify all these various interactions under consideration, if the cumulative effect of them is represented with a single interaction called the potential of mean forces (PMF). Recent Molecular Dynamics simulation studies by Schwierz et al [20] have shown that it is possible to express these PMFs between single ions and interfaces with an analytical LennardJones potential approach consisting of electrostatic and non-electrostatic interactions. However, these studies clearly show the complexity of the situation when it comes to explaining the cationic Hofmeister series on a polar surface such as ours. In addition to that, such calculations are quite complicated even for a single interface interacting with ions. For example, in $[20,21]$, the authors have focussed on a hydrophobic surface for calculating PMFs. Our wetting and adsorption mechanism, on the contrary, consists of two interfaces: a hydrophilic mica-water interface and a hydrophobic oilwater interfaces. The adsorption of various types of ions in the latter interface is reported to be quite complicated [52]. In the wake of the total complexity posed by the quantitative analysis of this problem, we have mainly concentrated on experimental evidence for the existence of a cationic wettability series, for our specific system of mica in ambient alkane.

It is worthwhile at this point to scrutinize the uniqueness of the wettability series demonstrated by our experiments. As mentioned earlier, when monovalent and divalent cations are written together in the order of finite contact angles, we obtain the series:

$$
\mathrm{Na}^{+}, \mathrm{K}^{+}<\mathrm{Li}^{+}<R b^{+}<C s^{+}<\mathrm{Ca}^{2+}<\mathrm{Mg}^{2+}<\mathrm{Ba}^{2+}
$$

while the classical (direct) Hofmeister series of cation adsorption on a negatively charged surface is given by: $\mathrm{Ca}^{2+}<\mathrm{Mg}^{2+}<\mathrm{Li}^{+}<\mathrm{Na}^{+}<\mathrm{K}^{+}<$ $\mathrm{Rb}^{+}<\mathrm{Cs}^{+}$. Among monovalent cations, the order is quite similar between classic Hofmeister series and our proposed wettability series except in the case of $\mathrm{Li}^{+}$. We have already explained the hydration effects for $\mathrm{Li}^{+}$cation, and relevant studies [20] have also found irregular behaviour in the PMF calculation of $\mathrm{Li}^{+}$, since the stability of the first or second hydration shell around the cation plays a significant role, e.g., Schwierz et al. have identified the stripping off of the hydration layer as well as the compression of the hydration shell as key parameters in $\mathrm{Li}^{+}$cation's strongest attraction to a hydrophilic medium.

Subsequently, we turn our attention to the divalent cations which according to the classic Hofmeister literature are supposed to adsorb less than various other monovalent cations onto a negatively charged surface. However, we 
have proposed and established [42] that in the presence of divalent cations, the surface charge of mica is reversed from negative to positive. The finite contact angle and hence, the stronger adsorption with divalent cations in our experiments can then directly be corroborated to the fact that on a cationic surface, the cations follow the reverse Hofmeister series, where $\mathrm{Mg}^{2+}$ or $\mathrm{Ca}^{2+}$ are deemed to adsorb most strongly.

The $\mathrm{pH}$ of the aqueous phase containing the cations also plays a role in the wettability order we have investigated. As seen from the contact angle maps (Figure 6.1, 6.2, 6.4, 6.5), wetting trends for both monovalent and divalent cations of smaller size $\left(\mathrm{r}_{0}\right.$, bare ion radius) depend on the $\mathrm{pH}$ values. The trend for the largest cations $\left(\mathrm{Cs}^{+}\right.$and $\mathrm{Ba}^{2+}$, on the other hand, are independent of the $\mathrm{pH}$ values, where already at a lower $\mathrm{pH}$ (3) the maximum contact angle displayed by the cation is observed. In an attempt to explain this trend, we first focus on the interaction of $\mathrm{Ba}^{2+}$ with $\mathrm{OH}^{-}$, which is related to the solubility of the hydroxide $\mathrm{Ba}(\mathrm{OH})_{2}$. In contrast to other hydroxides of divalent cations, e.g. $\mathrm{Mg}(\mathrm{OH})_{2}, \mathrm{Ba}(\mathrm{OH})_{2}$ is fairly soluble in water; its solubility product at $25^{\circ} \mathrm{C}$ is about $5 \times 10^{-3} \mathrm{~mol}^{3} / 1^{3}$, whereas that of $\mathrm{Mg}(\mathrm{OH})_{2}$ is about $2 \times 10^{-11} \mathrm{~mol}^{3} / \mathrm{l}^{3}$. Hence, at concentrations at the $\mathrm{mM}$ range, $\mathrm{Ba}^{2+}$ is not very likely to bind $\mathrm{OH}^{-}$, so that the activity of $\mathrm{Ba}^{2+}$ is hardly affected by $\mathrm{pH}$, and very little $\mathrm{Ba}(\mathrm{OH})^{+}$is generated. Once on the surface, $\mathrm{Ba}^{2+}$ will interact with the oxide sites involving deprotonation:

$$
\mathrm{X}-\mathrm{OH}+\mathrm{Ba}^{2+} \rightleftharpoons \mathrm{X}-\mathrm{OBa}^{+}+\mathrm{H}^{+}
$$

where, $X$ is an acidic site. Since, hardly any $\mathrm{Ba}(\mathrm{OH})^{+}$is present, the formation of $\mathrm{X}-\mathrm{O}-\mathrm{Ba}(\mathrm{OH})$ can be neglected and therefore the net charge on the surface is entirely controlled by $\mathrm{Ba}^{2+}$ adsorption as long as the surface acid is dissociated.

For monovalent cations in our study, hydroxide formation in solution can also be neglected. However, because they are monovalent, their binding to negative surface is much weaker than for divalent cations, and therefore the adsorption is sensitive to protonation of the surface sites:

$$
X-O H+M^{+} \rightleftharpoons X-O-M+H^{+}
$$

where, $\mathrm{M}$ is a monovalent cation. Only for the largest ion $\mathrm{Cs}^{+}$, the binding is sufficiently strong (enhanced by the dispersion forces) that it becomes insensitive to $\mathrm{pH}$ on the experimental range. As a result, wettability is also unaffected by $\mathrm{pH}$ for $\mathrm{Cs}^{+}$. 


\subsection{Concluding Remarks}

We have investigated and demonstrated wetting alteration of aqueous salt solution on a mineral surface in ambient alkanes. We observe that a transition from near-zero contact angle to a finite contact angle (up to $\sim 15^{\circ}$ ) occurs on mica as we follow a series of cations starting from $\mathrm{Na}^{+}, \mathrm{K}^{+}$to $\mathrm{Li}^{+}$, $\mathrm{Rb}^{+}, \mathrm{Cs}^{+}$and then to divalent cations $\left(\mathrm{Ca}^{2+}, \mathrm{Mg}^{2+} \mathrm{Ba}^{2+}\right)$. For the first time, we have reported an existing cationic order in relative wettability of mica in an oil-water system. We have introduced the term cationic Hofmesiter series of wettability alteration for this series.

For all these cases of transition to a finite contact angle, there is a threshold concentration above which this transition takes place, e.g., with $\mathrm{Ca}^{2+}$, it occurs around $50 \mathrm{mM}$, but for $\mathrm{Ba}^{2+}$ it occurs around $10 \mathrm{mM}$. We have conclusively shown in Chapter 4 that the wetting phase exists as a thin film, and have pointed out that the macroscopic wetting pattern in our experiments and especially the wetting alteration is intricately related to specific ion adsorption leading to various interactions in this film. The surface charge at the mica-water interface as well as oil-water interface plays a role in determining the electrostatic contribution in this film, as is evident from our streaming potential as well as AFM investigation. However, wetting alteration is not limited to the condition of charge-reversal at mica interface. The importance of ion polarizability, solvation energy, ion-charge density etc. in relative adsorption of ions overshadow the electrostatic effect. We acknowledge that this cationic wetting series is a unique ordering of cations for a specific mineral surface (mica) wetting in alkanes, since for a different substrate surface hydration, nature of charge on the substrate, the point of zero charge (pzc) and many other factors play a crucial role on the interaction with cations and subsequently, on the wetting properties. 


\section{Bibliography}

[1] F. Hofmeister. Zur lehre von der wirkung der salze. Archiv für experimentelle Pathologie und Pharmakologie, 24(4-5):247-260, 1888.

[2] H. Freundlich. Kapillarchemie. Akademische Verlagsgesellschaft, Leipzig, second edition edition, 1909.

[3] K. D. Collins and M. W. Washabaugh. The Hofmeister effect and the behaviour of water at interfaces. Quarterly Reviews of Biophysics, 18(04):323-422, 1985.

[4] M. G. Cacace, E. M. Landau, and J. J. Ramsden. The Hofmeister series: salt and solvent effects on interfacial phenomena. Quarterly Reviews of Biophysics, 30(03):241-277, 1997.

[5] P. Ball. Water as an active constituent in cell biology. Chemical Reviews, 108(1):74-108, 2008.

[6] W. Kunz. Specific Ion Effects. World Scientific, Singapore, 1st ed. edition, 2010.

[7] W. Kunz. Specific ion effects in colloidal and biological systems. Current Opinion in Colloid \& Interface Science, 15(1-2):34-39, 2010.

[8] W. Kunz, P. Lo Nostro, and B. W. Ninham. The present state of affairs with Hofmeister effects. Current Opinion in Colloid $\mathcal{E}$ Interface Science, 9(1-2):1-18, 2004.

[9] J. Lyklema. Simple Hofmeister series. Chemical Physics Letters, 467(4-6):217-222, 2009.

[10] Y. Zhang and P. S. Cremer. Chemistry of Hofmeister anions and osmolytes. Annual Review of Physical Chemistry, 61(1):63-83, 2010.

[11] M. Boström, W. Kunz, and B. W. Ninham. Hofmeister effects in surface tension of aqueous electrolyte solution. Langmuir, 21(6):2619-2623, 2005. 
[12] D. F. Parsons and B. W. Ninham. Charge reversal of surfaces in divalent electrolytes: The role of ionic dispersion interactions. Langmuir, 26(9):6430-6436, 2010.

[13] D. Horinek, A. Herz, L. Vrbka, F. Sedlmeier, S. I. Mamatkulov, and R. R. Netz. Specific ion adsorption at the air/water interface: The role of hydrophobic solvation. Chemical Physics Letters, 479(4-6):173-183, 2009.

[14] D. Ben-Yaakov and D. Andelmann. Revisiting the Poisson-Boltzmann theory: Charge surfaces, multivalent ions and inter-plate forces. Physica A, 389:2956, 2010.

[15] V. A. Parsegian and T. Zemb. Hydration forces: Observations, explanations, expectations, questions. Current Opinion in Colloid $\mathcal{E}$ Interface Science, 16(6):618-624, 2011.

[16] J. Lyklema. Lyotropic sequences in colloid stability revisited. Advances in Colloid and Interface Science, 100-102(0):1-12, 2003.

[17] M. Ricci, P. Spijker, and K. Voïtchovsky. Water-induced correlation between single ions imaged at the solid-liquid interface. Nat Commun, 5, 2014.

[18] T. Baimpos, B. R. Shrestha, S. Raman, and M. Valtiner. Effect of interfacial ion structuring on range and magnitude of electric double layer, hydration, and adhesive interactions between mica surfaces in $0.05-3$ $\mathrm{m} \mathrm{Li}^{+}$and $\mathrm{Cs}^{+}$electrolyte solutions. Langmuir, 30(15):4322-4332, 2014.

[19] R. Goldberg, L. Chai, S. Perkin, N. Kampf, and J. Klein. Breakdown of hydration repulsion between charged surfaces in aqueous $\mathrm{Cs}^{+}$solutions. Physical Chemistry Chemical Physics, 10(32):4939-4945, 2008.

[20] N. Schwierz, D. Horinek, and R. R. Netz. Reversed anionic Hofmeister series: The interplay of surface charge and surface polarity. Langmuir, 26(10):7370-7379, 2010.

[21] N. Schwierz, D. Horinek, and R. R. Netz. Anionic and cationic Hofmeister effects on hydrophobic and hydrophilic surfaces. Langmuir, 29(8):2602-2614, 2013.

[22] N. Schwierz and R. R. Netz. Effective interaction between two ionadsorbing plates: Hofmeister series and salting-in/salting-out phase diagrams from a global mean-field analysis. Langmuir, 28(8):3881-3886, 2012. 
[23] S. Berg, H. Ott, S. A. Klapp, A. Schwing, R. Neiteler, N. Brussee, A. Makurat, L. Leu, F. Enzmann, J. Schwarz, M. Kersten, S. Irvine, and M. Stampanoni. Real-time 3d imaging of haines jumps in porous media flow. Proceedings of the National Academy of Sciences of the United States of America, 110(10):3755-3759, 2013.

[24] J. Murison, B. Semin, J.C. Baret, S. Herminghaus, M. Schroeter, and M. Brinkmann. Wetting heterogeneities in porous media control flow dissipation. Physical Review Applied, 2(3), 2014.

[25] L. W. Lake. Enhanced Oil Recovery. Prentice Hall, 1989.

[26] X. Zhao, M. J. Blunt, and J. Yao. Pore-scale modeling: Effects of wettability on waterflood oil recovery. Journal of Petroleum Science and Engineering, 71(3-4):169-178, 2010.

[27] A. Muggeridge, A. Cockin, K. Webb, H. Frampton, I. Collins, T. Moulds, and P. Salino. Recovery rates, enhanced oil recovery and technological limits. Philosophical Transactions of the Royal Society aMathematical Physical and Engineering Sciences, 372(2006), 2014.

[28] Y. Li. Oil recovery by low salinity water injection into a reservoir: A new study of tertiary oil recovery mechanism. Transport in Porous Media, 90(2):333-362, 2011.

[29] J. J. Sheng. Critical review of low-salinity waterflooding. Jour. Pet. Sc. Eng., 120(0):216-224, 2014.

[30] G. Tang and N.R. Morrow. J. pet. sci. eng. 24:99, 1999.

[31] A. Lager, K. J. Webb, C. J. J. Black, M. Singleton, and K. S. Sorbie. Low salinity oil recovery - an experimental investigation1. Petrophysics, 49:28-35, 2006.

[32] P.G. de Gennes. Wetting: statics and dynamics. Rev. Mod. Phys., 57:827, 1985.

[33] D. Bonn, J. Eggers, J. Indekeu, J. Meunier, and E. Rolley. Wetting and spreading. Rev. Mod. Phys., 81:739, 2009.

[34] D. Bonn and D. Ross. Wetting transitions. Rep. Prog. Phys., 64:1085, 2001. 
[35] R. F. Kayser, J. W. Schmidt, and M. R. Moldover. Wetting layers and dispersion forces for a fluid in contact with a vertical wall. Physical Review Letters, 54(7):707-710, 1985.

[36] E. Cheng, Milton W. Cole, W. F. Saam, and J. Treiner. Helium prewetting and nonwetting on weak-binding substrates. Physical Review Letters, 67(8):1007-1010, 1991.

[37] J.W. Cahn. Critical point wetting. J. Chem. Phys., 66:3367, 1977.

[38] I. Ibagon, M. Bier, and S. Dietrich. Wetting in electrolyte solutions. The Journal of Chemical Physics, 138(21):214703, 2013.

[39] I. Ibagon, M. Bier, and S. Dietrich. Order of wetting transitions in electrolyte solutions. The Journal of Chemical Physics, 140(17):174713, 2014.

[40] F. Mugele and J. Baret. Electrowetting: from basics to applications. J. Phys. Cond. Matt., 17:R705, 2005.

[41] L. K. Koopal. Wetting of solid surfaces: Fundamentals and charge effects. Advances in Colloid and Interface Science, 179-182(0):29-42, 2012.

[42] F. Mugele, B. Bera, A. Cavalli, I. Siretanu, A. Maestro, M. Duits, M. A. Cohen-Stuart, D. Van Den Ende, I. Stocker, and I. Collins. Ion adsorption-induced wetting transition in oil-water-mineral systems. Scientific Reports, 5, 2015.

[43] P. K. Weissenborn and R. J. Pugh. Surface tension of aqueous solutions of electrolytes: Relationship with ion hydration, oxygen solubility, and bubble coalescence. Journal of Colloid and Interface Science, 184(2):550$563,1996$.

[44] A. P. dos Santos, A. Diehl, and Y. Levin. Surface tensions, surface potentials, and the Hofmeister series of electrolyte solutions. Langmuir, 26(13):10778-10783, 2010.

[45] R. Aveyard and S. M. Saleem. Interfacial tensions at alkane-aqueous electrolyte interfaces. Journal of the Chemical Society, Faraday Transactions 1: Physical Chemistry in Condensed Phases, 72(0):1609-1617, 1976.

[46] R. M. Pashley and J. N. Israelachvili. Dlvo and hydration forces between mica surfaces in $\mathrm{Mg}^{2+}, \mathrm{Ca}^{2+}, \mathrm{Sr}^{2+}$, and $\mathrm{Ba}^{2+}$ chloride solutions. Journal of Colloid and Interface Science, 97(2):446-455, 1984. 
[47] J. K. Beattie. The intrinsic charge on hydrophobic microfluidic substrates. Lab on a Chip, 6(11):1409-1411, 2006.

[48] J. Luetzenkirchen, T. Preocanin, and N. Kallay. A macroscopic water structure based model for describing charging phenomena at inert hydrophobic surfaces in aqueous electrolyte solutions. Phy. Chem. Chem. Phys., 10:4946, 2008.

[49] P. Kekicheff, S. Marcelja, T.J. Senden, and V.E. Shubin. Charge reversal seen in electrical double layer interaction of surfaces immersed in 2:1 calcium electrolyte. Jour. Chem. Phys., 99:6098, 1993.

[50] A. G. Volkov, S. Paula, and D. W. Deamer. Two mechanisms of permeation of small neutral molecules and hydrated ions across phospholipid bilayers. Bioelectrochemistry and Bioenergetics, 42(2):153-160, 1997.

[51] N. Kumar. Surface charge characterization of heterogeneous rock/clay materials. Thesis, 2016.

[52] D. E. Otten, P. R. Shaffer, P. L. Geissler, and R. J. Saykally. Elucidating the mechanism of selective ion adsorption to the liquid water surface. Proceedings of the National Academy of Sciences, 109(3):701-705, 2012.

[53] J. N. Israelachvili. 4 - Interactions Involving Polar Molecules, pages 71-90. Academic Press, San Diego, 2011.

[54] S. Loh and S. P. Jarvis. Visualization of ion distribution at the mica electrolyte interface. Langmuir, 26(12):9176-9178, 2010. 


\section{CHAPTER 7}

\section{Surfactant Induced Autophobing}

\section{Abstract}

Surfactant adsorption in a three-phase system and its influence on the wetting properties is relevant in various applications and has been widely studied. In this study we report a hitherto unobserved phenomenon, namely the retraction of an aqueous drop on solid substrates, caused by the deposition of species with a hydrophobic tail, that were dissolved in the ambient oil. This process is enabled by the formation of a reaction product between the amphiphilic molecules (fatty acid) and divalent cations $\left(\mathrm{Ca}^{2+}\right)$ at the oil/water interface. We investigate how this process depends on experimental parameters such as concentrations of the amphiphilic molecules and the solute, the chain lengths of the fatty acid and the alkane solvent and the substrate. Surfactant adsorption first occurs at the oil-water interface of the droplet, and subsequently, deposition on the solid phase takes place. AFM measurements confirm that surfactant is deposited on the solid by the moving contact line. This leads to a more hydrophobic substrate and a concomitant increase in contact angle, which we refer to as 'autophobing'. This phenomenon is observed on mica as well as on silica substrates, for several fatty acids and alkane solvents and thus appears to be a generic behavior. The phenomenon can be described with a theoretical model in which the rate of adsorption at the oil-water interface governs the overall kinetics of autophobing, and transfer to the solid is determined by a mass flux balance (similar to a Langmuir Blodgett transfer). The ensuing time-dependent oil-water and solid-oil interfacial tensions then produce the evolution of the contact angle.

Parts of this chapter appear as:

1. B. Bera, M.H.G. Duits, M.A. Cohen-Stuart, H.T.M. van den Ende \& F. Mugele. Fatty acid induced autophobing on mineral substrates. Soft Matter, Under Review (2016). 


\subsection{Introduction}

A host of modern applications in diverse disciplines require in-situ wettability alteration in the system and sometimes the need to park or move drops in a confined space. Examples include lab-on-a-chip devices for biomedical screening [1], immersion lithography [2], electrowetting platforms, oil recovery techniques etc. There are several methods to control the wettability and the dynamics of drops in such a system, namely, thermal excitation [3], drop manipulation using digital microfluidics and electrowetting [4], pressure driven flow for droplet-sorting [5] etc., but chemical driving of drops remains one of the most popular options in applications such as oil recovery since control via electric fields is not possible. Functional coating of the substrate is a primary ingredient for chemically driven dynamic droplets and this is often achieved by using surfactants. However, the adsorption of surfactants is often not limited to one interface. Also the interface of the drop with the ambient phase can recruit surfactants. This makes wettability alterations by surfactants more rich as a phenomenon, involving effects like Marangoni Effect [6, 7], dewetting [8, 9], self-propelling drops [10, 11, 12] and autophobing drops $[13,14,15,16]$.

Autophobing, i.e., the spontaneous retraction of the contact line of a drop (thereby increasing the contact angle) is one of the fascinating phenomena observed while studying wetting in the presence of amphiphilic molecules. In early studies on autophobing, the contact angles, adhesion energy and critical surface tension for drop spreading on hydrocarbon surfaces in the presence of alkanes were of strongest interest [14]. The principal mechanism suggested for autophobing was the self-assembly of surfactant molecules forming a monolayer on solid substrates [15], causing the retracting contact line of the drop. More recent studies [10,17] have focussed on autophobing of a liquid phase on another liquid subphase where the polar molecules from the spreading liquid phase escape through the three-phase contact line to form the thick layer of adsorbate at subphase/vapour interface and cause autophobing. The role of electrostatics and adsorption is often debated in the context of autophobing. However, experimental investigations confirm that irrespective of ionic or non-ionic surfactant, a self-assembled monolayer forms on the substrate because of the carryover of surfactant molecules across the contact line [18, 19]. Mathematical models have been developed to capture the phenomenon by lubrication theory [20,21, 22] or evolution theory for the density profile of the adsorbed layer [11, 12]. Among the many 
investigated mechanisms of autophobing (or a moving drop on a substrate) that have been investigated, two stand out: (i) the substrate changes its wettability through adsorption from the droplet phase and the adsorbed surfactant molecules do not return to the autophobing liquid phase (irreversible), and (ii) the autophobing drop removes the layer of surfactants deposited on the substrate and the substrate becomes bare again (reversible).

However, very few of these studies have focussed on a system with an ambient liquid phase and none of them have addressed the problem that the autophobing drop does not contain the surfactant itself. In this work, we consider such a system. Autophobing of aqueous drops on solid substrates is investigated, where the ambient oil contains an amphiphile, namely a fatty acid, and the aqueous drop contains dissolved salt. We study the mechanism of autophobing in this case, i.e, the manner in which the surfactant is deposited on the solid substrate in order to cause autophobing. We observe that metal stearate formation at the oil-water interface is a prerequisite for autophobing. We investigate the conditions, namely role of fatty acid concentration, salt concentration and $\mathrm{pH}$, for formation of interfacially active species. In order to support this understanding, we also develop a model involving the evolution kinetics and final equilibirum stage of the autophobing drop. The time-scale and the final contact angle are captured in fit parameters which in turn are connected to rate limiting processes and thermodynamic properties of adsorbed layers.

\subsection{Experimental Methods}

\subsubsection{Chemicals and Materials}

Anhydrous alkanes (heptane, decane, hexadecane, $>99 \%$, Sigma Aldrich) are the oil phases in the experiments. The oil is passed five times through a vertical column of Alumina powder $\left(\mathrm{Al}_{2} \mathrm{O}_{3}\right.$, Sigma Aldrich, Puriss grade $\left.>98 \%\right)$ to remove surface-active impurities until the interfacial tension (IFT) is constant $\sim 50 \mathrm{mN} / \mathrm{m}$ within $\pm 2 \mathrm{mN} / \mathrm{m}$ for at least 20 minutes. Different fatty acids (hexanoic, decanoic or octadecanoic acid, ACS reagent grade, Sigma Aldrich) are added to the alkanes at 1, 10, 100 or $1000 \mu \mathrm{M}$ concentrations in order to prepare the ambient phase. Ultrapure water from Millipore (Synergy UV Instruments, resistivity $18 \mathrm{M} \Omega$ ) is used for preparing salt solutions. We prepared all inorganic salt (Sigma Aldrich) solutions in a concentration 
range of $1 \mathrm{mM}$ to $1 \mathrm{M}$ by adding the water to carefully measured amount of the required salt. The $\mathrm{pH}$ of the solutions is adjusted between 2 and 10 using $\mathrm{HCl} / \mathrm{HNO}_{3}$ and $\mathrm{NaOH}(0.1 \mathrm{M}$, Sigma Aldrich). Muscovite mica (B\&M Mica Company Inc., USA; initial thickness $340 \mu \mathrm{m}$ ) and oxidized silicon wafers with an amorphous silicon oxide layer (thickness: $30 \mathrm{~nm}$ ) mimicking silica are the representative negatively charged solid surfaces. Mica sheets are cleaved inside the oil with scotch tape to obtain a pristine surface during the experiment. Silica surfaces are cleaned using piranha solution (mixture of $30 \%$ conc. $\mathrm{H}_{2} \mathrm{O}_{2}$ and $70 \%$ conc. $\mathrm{H}_{2} \mathrm{SO}_{4}$ ) followed by extensive rinsing with ultrapure water and plasma treatment.

\subsubsection{Optical Goniometry}

The wetting of aqueous drops on mica/silica is characterized by measurement of dynamic and equilibrium contact angles, using a contact angle goniometer (OCA 20L, Dataphysics Instruments GmBH, Germany). Before measuring the contact angles, interfacial tension (IFT) has been measured at oil-water interface using aqueous pendant drop of typical volume $20 \mu \mathrm{l}$ created in the ambient alkane phase (containing the fatty acids). Subsequently, aqueous drops of volume $2 \mu \mathrm{l}$ are created and quickly placed on the solid substrate. The time delay between the formation of the drop and its touching down is kept within $1 \mathrm{~s}$. The time-dependant contact angle of the drop is extracted from video snapshots using the tangent and circle-fitting data analysis software (SCA 22) provided with the instrument. Contact angles can be determined with an accuracy of $\pm 1^{\circ}$. The minimum contact angle that can be determined on reflective surfaces is $\sim 2^{\circ}$. The measurement of contact angle is repeated 25 times (drops) placed on at least 5 different solid substrates. Various technical aspects of our contact angle measurements are discussed in detail in Chapter 3.

\subsubsection{Atomic Force Microscopy}

An atomic force microscope (Dimension Icon with Bruker AXS Multimode 8, USA) is used to investigate the adsorption of various species on the mineral surface and the topography of these adsorbed layers. These experiments are performed ex-situ in air after the substrate is removed from ambient oil and the autophobed drop is gently blown away. All experiments are done in tapping mode with ScanAsyst fluid silicon probes (Bruker) with sharp tips and the images are collected with ScanAsyst Air software provided with the 
instrument. Nominal cantilever and tip properties are: tip radius $<10 \mathrm{~nm}$; tip height, $0.5-0.75 \mu \mathrm{m}$; cantilever spring constant $0.4 \mathrm{~N} / \mathrm{m}$. The AFM is operated under ambient conditions with small cantilever oscillation amplitudes $(<10 \mathrm{~nm})$ and weak damping (i.e. high amplitude setpoint values) to protect both tip and sample against damage. Around 3-4 topography and phase images are recorded at various neighbouring locations outside the final contact line of the autophobed drop. Image analysis is performed using Bruker's standard Nanoscope Analysis 1.4 package.

\subsection{Results}

\subsubsection{Autophobing}

Our investigations comprise of a number of simple aliphatic fatty acids dissolved in different alkane oils using several series of aqueous drops with $\mathrm{NaCl} / \mathrm{CaCl}_{2}$ dissolved at different concentrations. We will focus on the results for 1-octadecanoic acid $\left(\mathrm{C}_{18} \mathrm{H}_{36} \mathrm{O}_{2}\right)$ (commonly known as stearic acid, henceforth abbreviated as SA) dissolved in decane in the following sections. The wetting behaviour and adsorption phenomena were found to be comparable for other fatty acids and alkanes, which are discussed in the Appendix.

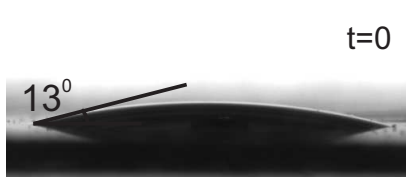

(a)

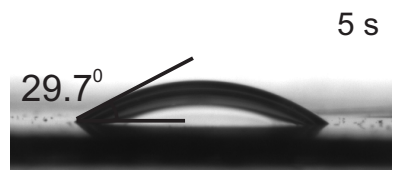

(b)

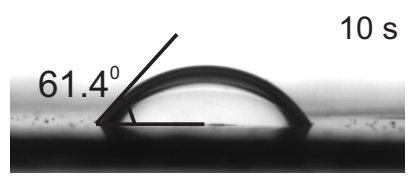

(c)

Figure 7.1: An autophobic drop; Screen-shots showing the gradual increase of contact angle for a $1 \mathrm{M} \mathrm{CaCl}_{2}$ aqueous drop at $\mathrm{pH} 8$ on mica in decane with $100 \mu \mathrm{M}$ stearic acid at (a) $0 \mathrm{~s}$ (b) $5 \mathrm{~s}$ and (c)10 s.

The behaviour of aqueous drops of $\mathrm{CaCl}_{2}$ on a mineral surface in ambient oil containing SA (concentrations between $1 \mu \mathrm{M}$ to $100 \mu \mathrm{M}$ in oil) is very different from that without any SA in oil. For drops containing $\mathrm{NaCl}$, the contact angle $\theta$ increases slightly compared to the unmeasurable contact angles $\left(<2^{\circ}\right)$ that we reported in Chapter 4 , but never exceeded $10^{\circ}$. For drops containing $\mathrm{CaCl}_{2}$ at $\mathrm{pH}$ higher than 5 , the aqueous drop initially assumes 
a contact angle $\theta \sim 10^{\circ}$, as in the absence of SA. Within seconds, however, there is a spontaneous inward motion of the three-phase contact line and $\theta$ increases to values of up to $70^{\circ}$. We will refer to this spontaneous increase of contact angle (along with the retraction of contact line) as 'autophobing'. Figure 7.1 shows an example: screen shots of a $1 \mathrm{M} \mathrm{CaCl}_{2}$ drop on mica in ambient decane containing $100 \mu \mathrm{M}$ stearic acid. On a typical time-scale of $10 \mathrm{~s}$, this gradual increase of contact angle occurs when $>10 \mathrm{mM}$ of $\mathrm{CaCl}_{2}$ is present in the aqueous phase.

In the concentration range of 10 to $100 \mathrm{mM}$, we found that the growth of the contact angle, i.e., 'autophobing', is often accompanied by 'self-propelling' of the aqueous droplets, i.e., as the contact angle begins to increase during autophobing, drops spontaneously move on the solid substrate in an arbitrary direction. After the drop stops moving, it undergoes further autophobing on the new spot. The propelling of the drop clearly highlights and magnifies the non-uniform wettability around the drops. In this chapter we will only focus upon the autophobing of drops that do not show significant displacement. Self propulsion is considered as a manifestation of autophobing, in combination with a heterogeneous substrate.

\subsubsection{Extent of Wettability Alteration}

The deposition of an aqueous drop leads to a final equilibrium contact angle on the solid phase. These contact angles are shown in Figure 7.2, for $100 \mu \mathrm{M}$ SA in decane, with aqueous drops of $\mathrm{CaCl}_{2}$ on mica and silica. The final contact angles on mica and silica are quite similar. The datapoints at $\mathrm{pH}<$ 6 do not display autophobing i.e., when $\mathrm{CaCl}_{2}$ droplets at $\mathrm{pH} 3,4$ and 5 are placed on the substrate, we observe contact angles which remain constant over time. Between pH 6 and 9, the deposited droplets assume an initial contact angle, but autophobe within 10-15 s to reach at a much higher contact angle (shown in the figure). We observe a jump in the contact angle $\theta$ from $\sim 10^{\circ}$ to $\sim 70^{\circ}$, as we cross the threshold $\mathrm{pH} 5$. It is noteworthy, that the $1 \mathrm{M} \mathrm{CaCl}_{2}$ drops at $\mathrm{pH} 5$ have a larger contact angle compared to those at $\mathrm{pH} 3$ or 4 , but no time dependence of contact angle (autophobing) was observed for these drops.

For autophobing drops, the final contact angle shows a clear dependence on the salt concentration in the aqueous phase, where lower equilibrium contact angles are observed for $100 \mathrm{mM}, 10 \mathrm{mM}$ and $1 \mathrm{mM}$ (compared to 1 

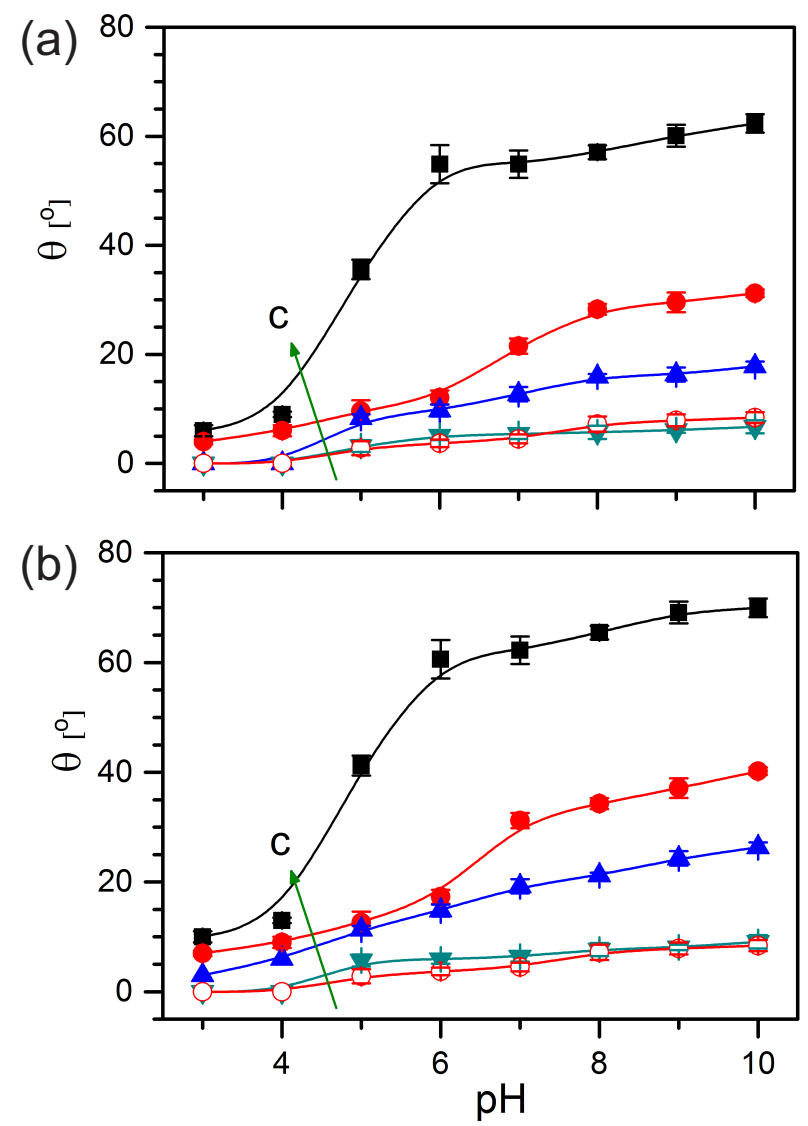

Figure 7.2: Equilibrium contact angles on (a) mica and (b) silica (open symbols); in ambient decane with stearic acid vs. $\mathrm{pH}$ for various concentrations of $\mathrm{CaCl}_{2}$ : $1 \mathrm{mM}$ (cyan downward triangles), $10 \mathrm{mM}$ (blue upward triangles), $100 \mathrm{mM}$ (red circles), $1 \mathrm{M}$ (black squares), and $100 \mathrm{mM} \mathrm{NaCl}$ (red open symbols). Stearic acid concentration: $100 \mu \mathrm{M}$. Each datapoint is an average of 25 independent drops under same conditions with the standard deviation shown in the error bar. The solid lines are guides to the eye. The arrow and the letter ' $c$ ' depict increasing salt concentration.

M) $\mathrm{CaCl}_{2}$ concentrations. Figure 7.3 shows the final contact angles for drops of these salt concentrations at $\mathrm{pH} 9$, at SA concentrations $100 \mu \mathrm{M}$ and $1 \mathrm{mM}$. We recall here, that for $\mathrm{NaCl}$ solution irrespective of salt concentration and $\mathrm{pH}$, there is no autophobing and no contact angle $>10^{\circ}$ is observed. This underlines that the autophobing is related to the calcium and not the ionic 
strength in the aqueous drops.

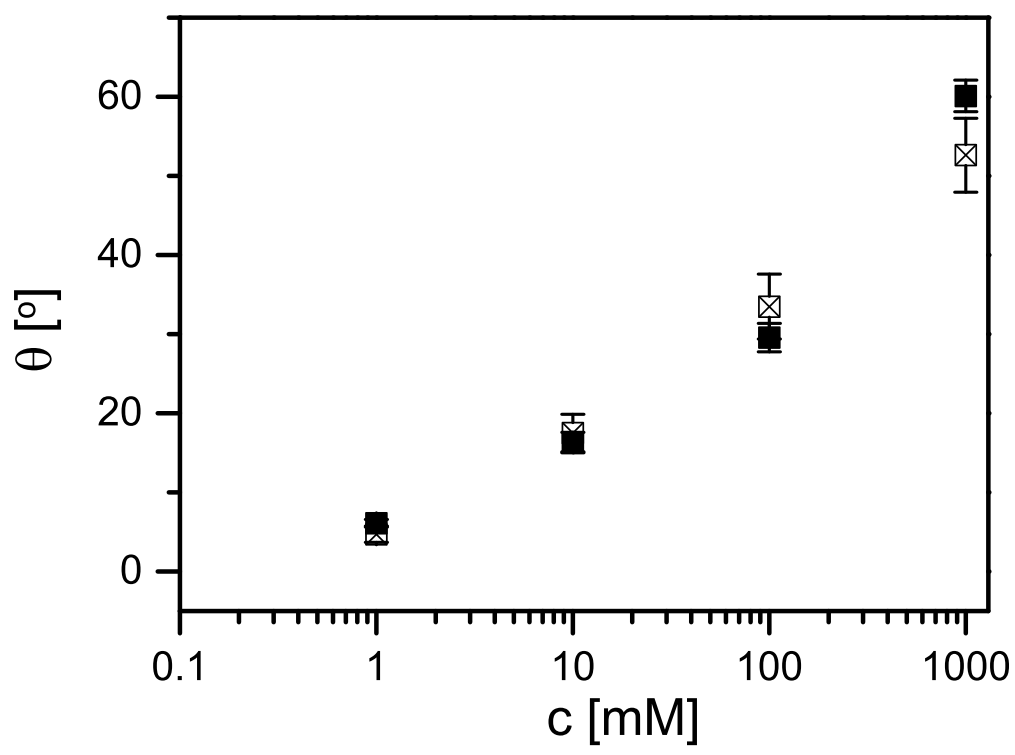

Figure 7.3: Equilibrium contact angles for autophobing drops of various $\mathrm{CaCl}_{2}$ concentrations at $\mathrm{pH}$ 9; drops are on mica in ambient decane with stearic acid at various concentrations: $100 \mu \mathrm{M}$. (closed symbols) and $1 \mathrm{mM}$ (crossed symbols). Each datapoint is an average of 25 independent drops under same conditions with the standard deviation shown in the error bar.

\subsubsection{Evolution of Contact Angle}

Along with the final equilibrium contact angle after autophobing, the evolution of the contact angle i.e., the time-scale over which the autophobing occurs is also interesting. We observe that this time-scale depends on the bulk SA concentration in the ambient oil. As this concentration increases, the autophobing becomes quicker, and vice versa, e.g., the autophobing time scale is $\sim 30-40 \mathrm{~s}$ for $100 \mu \mathrm{M} \mathrm{SA}$, while for $1 \mathrm{mM} \mathrm{SA}$, it is $\sim 10 \mathrm{~s}$. The time-scale does not depend on $\mathrm{CaCl}_{2}$ concentration in the aqueous phase: as long as the SA concentration in the oil phase is kept constant, drops of $1 \mathrm{M}$ or $100 \mathrm{mM}$ $\mathrm{CaCl}_{2}$ autophobe over similar time, $\sim 50-60 \mathrm{~s}$. We observe variations in the dynamic contact angle trends, i.e., there are experimental irreproducibilities. Even though experimental variables have been carefully controlled, the data 


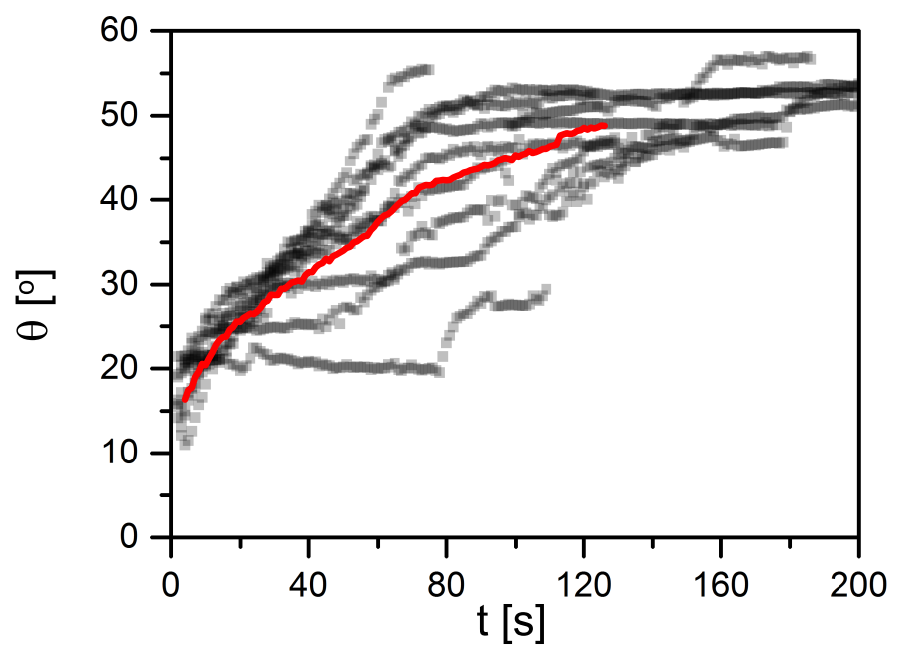

Figure 7.4: Contact angle evolution for $1 \mathrm{M} \mathrm{CaCl}_{2}$ drops on mica in ambient decane containing $100 \mu \mathrm{MSA}$. The evolution trend is the average of 15 identical drops (individual trends shown as scatter in the background).

fluctuate around this average, indicating that the motion of the contact line is not smooth, most likely due to pinning/de-pinning incidents. This probably also causes irregular deposition of surfactant on the solid, which could be the reason for the aforementioned self-propulsion exhibited by some drops. This scatter is depicted in Figure 7.4, where contact angle evolution trends for 15 drops of $1 \mathrm{M} \mathrm{CaCl}_{2}$ solution (all at $\mathrm{pH} 8$ ) are shown (in the background with light grey scatter). The average of these 15 curves is represented in red. The behavior of individual drops clearly deviates from each other, but using the average evolution trend, we get a reasonably good approximation of the slope and final plateau (equilibrium contact angle) for a specific condition. We will discuss these trends further in the model section and in Figure 7.9.

\subsubsection{Generality of the Phenomenon}

We notice in Figure 7.2 that the autophobing and the equilibrium contact angles are remarkably similar for mica or silica. This suggests that for negatively charged mineral surfaces such as mica and silica, the spontaneous contact angle increase is mainly determined by the adsorption of the polar components aided by a divalent ion and that other details of the substrate 
do not play a key role. Systems with other fatty acids (hexanoic acid, decanoic acid) also behave in similar manner, though the final contact angle depends somewhat on the aliphatic chain length. The length of the linear alkane acting as solvent for the fatty acids seems to have minimal effect on the experiments, since changing from decane to heptane or hexadecane has a minor effect on the contact angle trend. There is no change in the autophobing behaviour upon changing the fatty acids or the solvent alkanes. These results are presented and discussed in Appendix I. In the following section, we will focus on the mechanistic explanation of autophobing, starting from the molecular-scale events such as the adsorption of SA at various interfaces.

\subsection{Autophobing Mechanism}

\subsubsection{Surfactant Adsorption}

A change in wettability (i.e., the contact angle) can be considered the result of changes in interfacial energies. The Young's equation is written as: $\cos \theta=\frac{\gamma_{s o}-\gamma_{s w}}{\gamma_{o w}}$, where, $\gamma_{o w}, \gamma_{s o}$ and $\gamma_{s w}$ are the interfacial tensions of the oil-water, solid-oil and solid-water interfaces, respectively. Unlike the case of ambient oil without a polar component (dashed line in Figure 7.5 represents IFT at deionised water-decane interface) such as in Chapter 4 and 6 , in these experiments there is a significant time-dependant decrease in the oil-water interfacial tension. We have carried out IFT measurements with pendant drops. Figure 7.5 shows example measurements for aqueous drops of various $\mathrm{CaCl}_{2}$ concentrations (at $\mathrm{pH}$ 8) in ambient decane containing 100 $\mu \mathrm{M}$ SA. At a relatively high salt concentration $\left(1 \mathrm{M} \mathrm{CaCl}_{2}\right)$, there is a sharp drop of the interfacial tension $\gamma_{\text {ow }}$ over a few seconds and then the gradual attaining of a plateau, while at lower salt concentration a mild decrease of the IFT is observed. Similar trends have also been observed in previous studies [23].

If $\gamma_{o w}$ is the only interfacial tension changing during the autophobing process, then we would have seen a decrease in the contact angle (from Young's relation). Instead we observe a gradual increase in the contact angle up to a value as high as $70^{\circ}$. Hence, it is implied that $\gamma_{s o}-\gamma_{s w}$ must decrease even more compared to $\gamma_{\text {ow }}$. Assuming that SA cannot be transported through the drop phase (implying $\gamma_{s w}$ as constant), we attribute this drastic 
change of contact angle to a decrease in the solid-oil interfacial tension $\left(\gamma_{s o}\right)$, which occurs because of the adsorption of calcium stearate (Ca-SA).

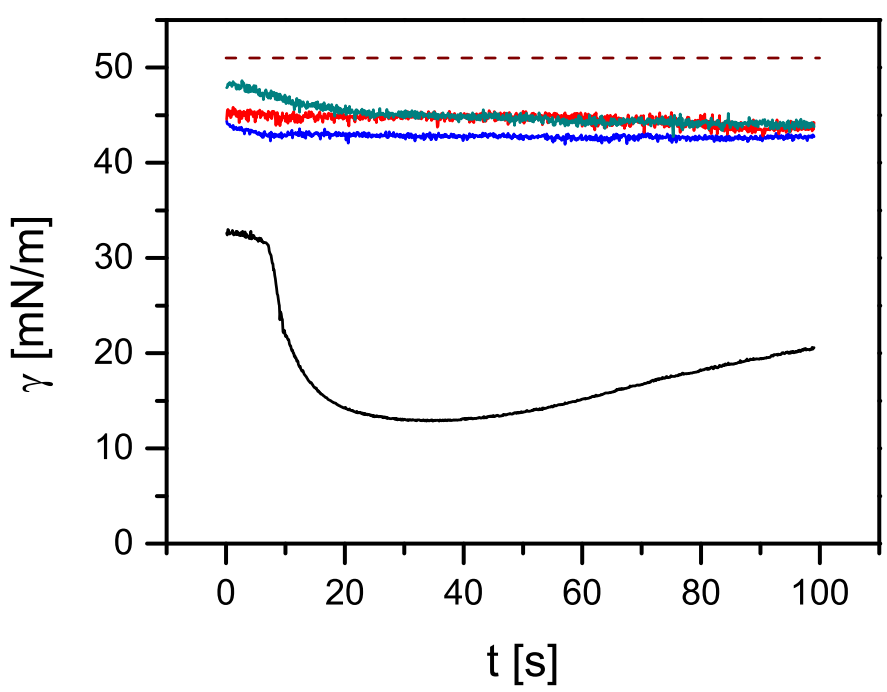

Figure 7.5: Interfacial tension (IFT) w.r.t time at water/oil (decane with $100 \mu \mathrm{M}$ $\mathrm{SA}$ ) for pendant drops of various $\mathrm{CaCl}_{2}$ concentration: $1 \mathrm{mM}$ (dark cyan), 10 $\mathrm{mM}$ (red), $100 \mathrm{mM}$ (blue) and $1 \mathrm{M}$ (black). All drops are at $\mathrm{pH}$ 8. A reference IFT measurement is shown for deionised water-decane interface when there is no surfactant present in decane (brown dashed line).

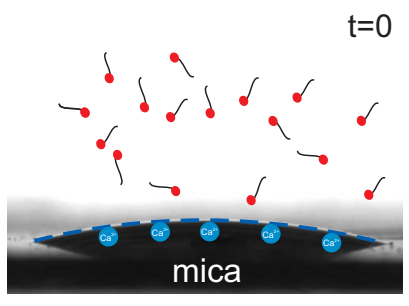

(a)

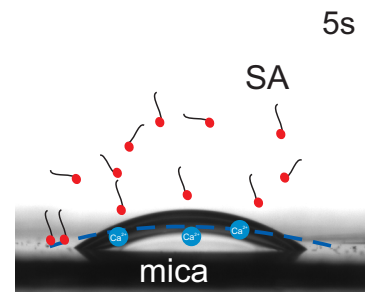

(b)

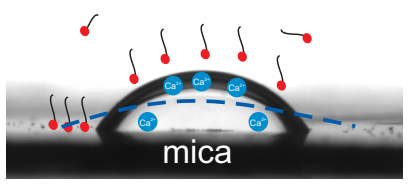

(c)

Figure 7.6: Proposed adsorption mechanism leading to the autophobing/selfpropelling at various time-stages (a) $0 \mathrm{~s} \mathrm{(b)} 5 \mathrm{~s} \mathrm{(c)} 10 \mathrm{~s}$ after placing the drop on substrate (mica). Ca-SA formation (Calcium:blue, SA:red head-groups) is depicted at the interface. 
During the adsorption of SA onto the solid substrate, the aqueous drop plays an imperative role as implied by the clear dependence of the autophobing phenomenon on the composition of the aqueous phase. The deprotonated SA molecule has a negatively charged headgroup $\left(\mathrm{R}-\mathrm{COO}^{-}\right.$, depicted red in Figure 7.6) and cannot get adsorbed to the negatively charged mica or silica substrates directly. We have observed that both high $\mathrm{Ca}^{2+}$ (blue in Figure 7.6) concentration and $\mathrm{pH}$ are prerequisites for autophobing. This indicates, in line with previous findings [23, 24], that the deprotonation of SA can only take place at the $\mathrm{O} / \mathrm{W}$ interface at a high $\mathrm{pH}$ and salt concentration.

Hence formation of calcium stearate at $\mathrm{O} / \mathrm{W}$ interface is the enabling step for Ca-SA getting adsorbed to the $\mathrm{S} / \mathrm{O}$ interface. Following diffusion from the bulk, SA gets adsorbed to the $\mathrm{O} / \mathrm{W}$ interface first. It is plausible that the $\mathrm{O} / \mathrm{W}$ interface acts as the reservoir of calcium stearate, from which it gets delivered to the $\mathrm{S} / \mathrm{O}$ interface. As the adsorption sites on the oil-water interface gradually get filled with $\mathrm{SA}$, there is subsequently a transfer of these SA molecules along the $\mathrm{O} / \mathrm{W}$ interface onto the solid phase. $\mathrm{Ca}^{2+}$ acts as the bridge between the negatively charged headgroup(s) in SA molecule and the negatively charged solid surface. The autophobing of our drops implies that while the contact line recedes, surfactant material keeps getting deposited. Otherwise the Ca-SA deposition would have stopped the spreading of the aqueous drop, but would not have led to the inward retraction of the contact line.

Following this discussion, the key ingredients of autophobing are clearly identified as the adsorption of calcium stearate at relatively high $\mathrm{pH}$ on mi$\mathrm{ca} /$ silica surface leading to a gradual increase of contact angle. A proposition has been made for the pathways (diffusion, formation of metal searate at $\mathrm{O} / \mathrm{W}$ interface and transfer of stearate to solid substrate). However, various mechanistic details of the process are still missing, and in the following sections through experiments and modeling, we are going corroborate the various propositions.

\subsubsection{Surfactant Deposition}

We have used atomic force microscopy (AFM) to investigate the adsorbed surfactant molecules on the solid substrates. We found no evidence of adsorbed SA on the substrate inside the final three-phase contact line of the 
drop. Hence, our proposition that water-insoluble SA does not get adsorbed under the drop is confirmed. AFM imaging outside the three-phase contact line corroborates our proposed mechanism for autophobing behavior of the aqueous drops: the surface is covered by a stearate monolayer, very similar to the partially decomposed Langmuir-Blodgett films of SA reported in earlier investigations [25].

(a)

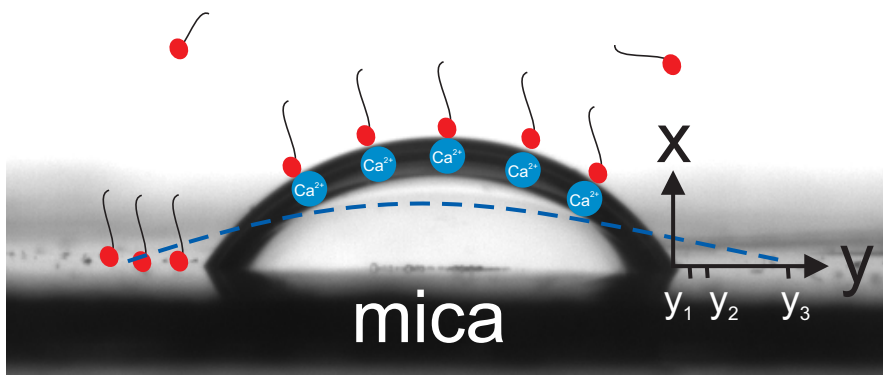

(b)
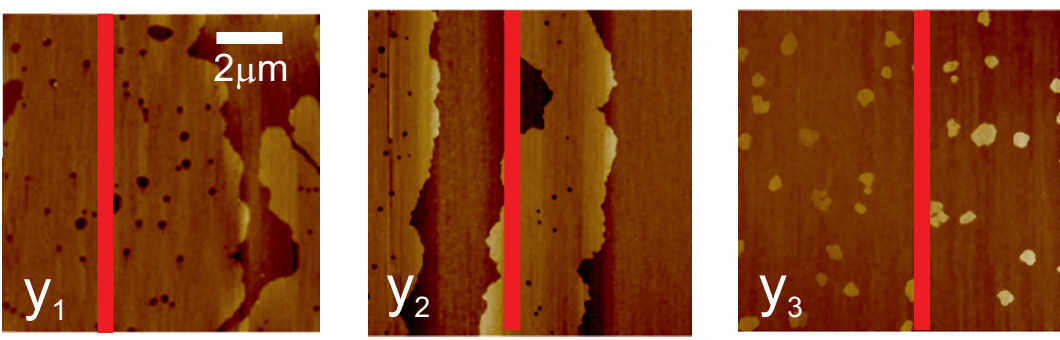

(c)
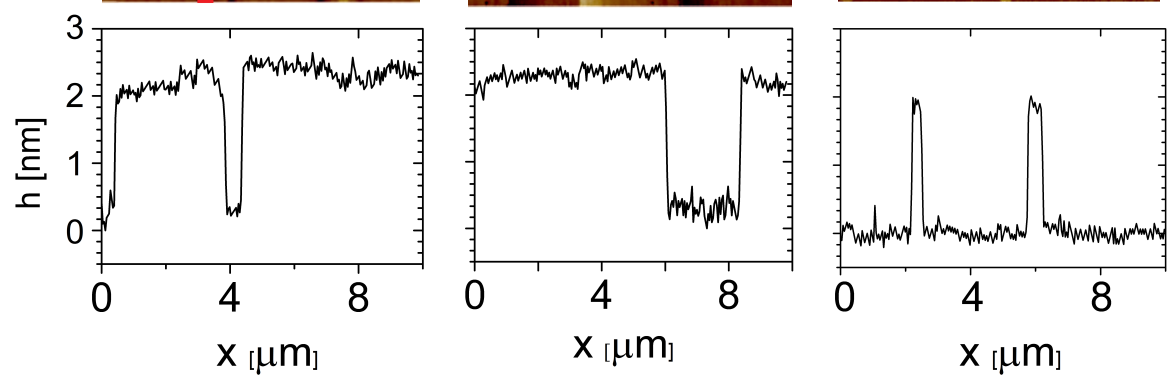

Figure 7.7: Atomic Force Microscopy reveals the layer thickness and density next to a $1 \mathrm{M} \mathrm{CaCl}_{2}$ drop deposited on mica in ambient decane with $100 \mu \mathrm{M}$ SA. (a) The drop after autophobing; the initial shape of the drop is shown by the dashed blue line and schematics of SA molecules in bulk and in adsorbed state are shown (b) The AFM thickness map is shown at various distances away from this 'autophobing' drop: (left) $100 \mu \mathrm{m}$ (centre) $200 \mu \mathrm{m}$ (right) $800 \mu \mathrm{m}$. Dark brown signifies the bare substrate while the patches of lighter brown represents the adsorbed SA layer (c) Height profiles corresponding to the thickness maps in (b). 
In Figure 7.7a, we show an example drop after autophobing where the SA (red) molecules as well as the initial contact line before autophobing (blue) are depicted schematically. The final position of the contact line is considered as the origin for AFM experiments, and the adsorption of SA is investigated at various distances away (radially outward) from this origin. Figure 7.7b shows the thickness maps at three representative distances. Close to the final contact line of the droplet, the SA layer is dense with occasional holes; At an intermediate distance $\sim 0.5 \mathrm{~mm}$ away from the contact line, the patches of adsorbed layer are less dense implying a medium coverage of adsorbed SA. Farther away, bare mica was seen with occasional islands of monolayer stearate. Hence, we observe a gradient in the amount of adsorbed SA, decreasing gradually as we move away from the contact line. Assuming that the measured topography is an adequate representation of the Ca-SA as it was deposited in the wet stage of autophobing, the gradient suggests that the transport of SA from oil-water interface onto the solid substrate gradually increases while the drop is autophobing. As the oil-water interface gets slowly saturated by SA molecules, it deposits more oncoming fatty acid molecules on the solid substrate.

Based on these mechanistic steps, we will build up a simple adsorption model in the following section, which captures the salient features of the autophobing drops, and investigates the parameters governing this phenomenon.

\subsection{Theoretical Model}

We set up our theoretical model with the goal of learning about the kinetics of the adsorption process and the rate determining steps as well as reproducing the observed evolution of the contact angle, using a minimum number of (plausible) assumptions.

The aqeuous drop is considered to be of spherical cap geometry during the entire process of autophobing. Hence the radius of curvature of the drop as well as the radius of the contact line is a function of contact angle $\theta$ (see Appendix II). The contact angle of the autophobing drop at a specific timeinstant $t$ is assumed to be the result of an instantaneous mechanical (but not chemical) equilibrium, where both the $\mathrm{O} / \mathrm{W}$ and the $\mathrm{S} / \mathrm{O}$ interfacial tension decrease over time due to a progressive accumulation of surface active ma- 
terial leading to higher densities. Here, the first step is the formation of a surfactant layer at the oil-water interface (Figure 7.8a, left) via diffusion of SA through the ambient solution followed by an adsorption step leading to formation of Ca-SA. The transfer of Ca-SA to the solid substrate starts as soon as the surfactant layer forms at the $\mathrm{O} / \mathrm{W}$ interface, and this transfer is considered to occur along the surface of the spherical cap.

We assume that $N$ molecules are adsorbed in the $\mathrm{O} / \mathrm{W}$ interface. The area of the $\mathrm{O} / \mathrm{W}$ interface is $A$ and the length of the contact line is $L=2 \pi r$ where $r$ is the radius of the circular contact line. Hence, the surface densities at the $\mathrm{O} / \mathrm{W}$ and $\mathrm{S} / \mathrm{O}$ interfaces are $\Gamma_{1}$ (which can be expressed as $\Gamma_{1}=N / A$ and $\Gamma_{2}$, respectively. The contact angle $\theta$ of the drop increases from a minimum value $\theta_{\min }$ to a maximum value $\theta_{\max }$ after the autophobing.
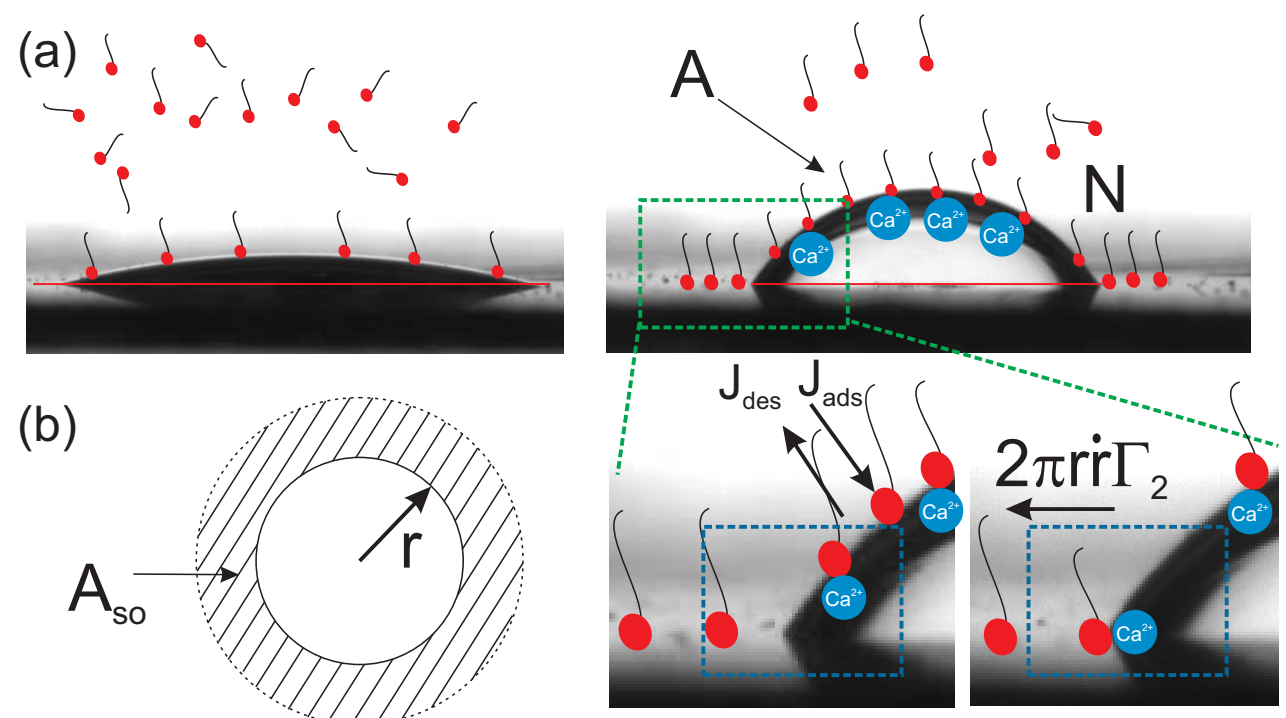

(b)

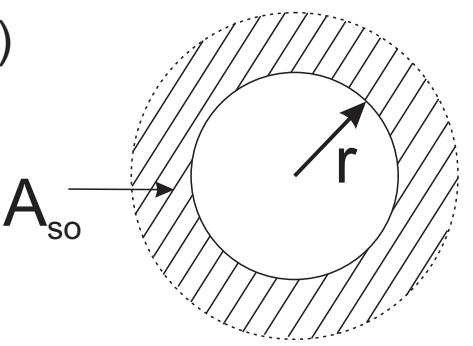

Figure 7.8: Stearic acid adsorption occurs first from bulk ambient alkane phase to the oil-water interface (a, left) and then subsequently Ca-SA get deposited on the solid substrate (a,right). The enlarged depiction of this deposition process is shown in b, right, where the control volume (area within blue dashed line) is shown along with the various influx and outflux terms considered in the model. (b, left) stearic acid coverage (hatched area) on a solid substrate for an autophobing drop. The intitial three-phase contact line is depicted by the dashed line, while the receding contact line is shown by the solid line.

The contact angle $\theta$ at a time $t$ is expressed following Young's relation: 


$$
\cos \theta(t)=\frac{\gamma_{s o}(t)-\gamma_{s w}}{\gamma_{o w}(t)}
$$

where, $\gamma(t)$ is the instantaneous interfacial tension. The surface tensions depend on time via the surface coverages $(\Gamma)$ :

$$
\cos \theta(t)=\frac{\gamma_{s o}\left(\Gamma_{2}\right)-\gamma_{s w}}{\gamma_{\text {ow }}\left(\Gamma_{1}\right)}
$$

Since the instantaneous equilibrium holds only in the mechanical sense, we cannot express the dependence of IFT on surface coverage using standard equations of state such as Gibbs equation, which only holds at a chemical equilibrium. In absence of more detailed information we write a linear relation between the instantaneous interfacial tension and surface density:

$$
\begin{aligned}
\gamma_{o w} & =\gamma_{o w, 0}-\alpha_{1} \Gamma_{1} \\
\gamma_{s o} & =\gamma_{s o, 0}-\alpha_{2} \Gamma_{2}
\end{aligned}
$$

where $\gamma_{0}$ is the initial interfacial tension of the bare interface. Assuming that the initial contact angle for an autophobing drop is $\theta_{0}$, we can write from Young's equation: $\gamma_{s o}-\gamma_{s w}=\gamma_{o w, 0} \cos \theta_{0}-\alpha_{2} \Gamma_{2}$, where the S/W interfacial tension is assumed to remain unchanged i.e., $\gamma_{s w}=\gamma_{s w, 0}$. This relation also implies that $\Gamma_{2}=0$ at $t=0$, i.e., the $\mathrm{S} / \mathrm{O}$ interface does not have any adsorbed stearate at the beginning. In the following derivations, we will use $\gamma_{0}$ to represent $\gamma_{o w, 0}$, the oil-water interfacial tension when autophobing starts. Hence, we can write:

$$
\cos \theta(t)=\frac{\gamma_{0} \cos \theta_{0}-\alpha_{2} \Gamma_{2}(t)}{\gamma_{0}-\alpha_{1} \Gamma_{1}(t)}
$$

Next, we consider the time dependences of $\Gamma_{1}$ and $\Gamma_{2}$. Figure $7.8 b$, right, depicts our chosen control volume around a small section of the threephase contact line, in which we consider all the influx and outflux of the SA molecules for $\mathrm{O} / \mathrm{W}$ and $\mathrm{S} / \mathrm{O}$ interfaces. Based on these fluxes, we can formulate the following rate equation:

$$
\frac{d N}{d t}=A\left(J_{\mathrm{ads}}-J_{\mathrm{des}}\right)+2 \pi r \dot{r} \Gamma_{2}
$$

where, $J_{a d s}$ and $J_{d e s}$ are the adsorption and desorption fluxes of SA molecules from the bulk oil phase to the $\mathrm{O} / \mathrm{W}$ interface, respectively. $2 \pi r \dot{r} \Gamma_{2}$ denotes 
the outward flux of SA molecules from $\mathrm{O} / \mathrm{W}$ interface along the spherical cap surface. These molecules, which are deposited on the S/O interface along the contact line, are assumed to stick to the substrate and not move inwards with the contactline i.e., the transfer is Langmuir-Blodgett (LB) like deposition. It is important to note that $\dot{r}$, i.e., the contact line velocity, is negative since the drop moves inward while autophobing. The fluxes are defined by:

$$
\begin{aligned}
& J_{\mathrm{ads}}=c k_{1}\left(\Gamma_{\infty}-\Gamma_{1}\right) \\
& J_{\mathrm{des}}=k_{2} \Gamma_{1}
\end{aligned}
$$

where $c$ is the local concentration of the stearate molecules in the oil phase near the oil-water interface and $k_{1}$ and $k_{2}$ are rate constants associated with adsorption and desorption at this interface, respectively. We assume that the desorption of SA molecules from the oil-water interface back to the bulk oil phase is negligible; i.e., $k_{2} \sim 0$. Hence, equation 7.6 becomes:

$$
\frac{d N}{d t}=A J_{\mathrm{ads}}+2 \pi r \dot{r} \Gamma_{2}
$$

Furthermore, we assume that the deposition of Ca-SA from the O/W interface to the $\mathrm{S} / \mathrm{O}$ interface involves a complete and instantaneous transfer of only the surfactants within the control box; in other words, any unevenness in the local surface density $\Gamma_{2}$ caused by the loss of molecules at the contact line is neglected (Langmuir Blodgett type deposition). The deposition, thus, is completely controlled by the fact that as the drop autophobes, the surface area A of the spherical cap reduces, leaving the extra SA molecules outside the cap which are then deposited on the solid substrate. We represent this assumption by the following expression:

$$
\Gamma_{1} \dot{A}-2 \pi r \dot{r} \Gamma_{2}=0
$$

Combining equations 7.9 and 7.10, we obtain:

$$
\dot{\Gamma}_{1}=J_{a d s}=c k_{1}\left(\Gamma_{\infty}-\Gamma_{1}\right)
$$

On the other hand, from equation 7.10 and the spherical cap shape of the drop, we obtain the relation between $\Gamma_{1}$ and $\Gamma_{2}$ (see Appendix II for the derivation):

$$
\Gamma_{2}=\frac{\dot{A}}{2 \pi r \dot{r}} \Gamma_{1}=\cos \theta \Gamma_{1}
$$


Based on Equations 7.5 and 7.12, we can write the implicit equation relating $\theta$ and $t$ as follows:

$$
\cos \theta(t)=\frac{\cos \theta_{0}}{1+\frac{\alpha_{2}-\alpha_{1}}{\gamma_{0}} \Gamma_{1}(t)}
$$

The time-dependent $\mathrm{O} / \mathrm{W}$ surface coverage $\Gamma_{1}$ can be obtained from equation 7.11:

$$
\Gamma_{1}=\Gamma_{1}^{\infty}\left(1-e^{-\left(c k_{1}\right) t}\right)
$$

Hence, Equation 7.13 can be written as:

$$
\cos \theta(t)=\frac{\cos \theta_{0}}{1+\frac{\alpha_{2}-\alpha_{1}}{\gamma_{0}} \Gamma_{1}^{\infty}\left(1-e^{-t / \tau}\right)}
$$

where, $\tau=\left(c k_{1}\right)^{-1}$. This equation suggests that autophobing will continue as long as $\Gamma_{1}$ keeps on growing. Bulk concentration $c_{\infty}$ and diffusion, thus, do not necessarily control the rate of autophobing. The local (sublayer) concentration $c$ (which would be proportional to the bulk concentration) determines the time-scale $\tau$ of autophobing together with a rate constant which could be determined by either diffusion or an adsorption (reaction) energy barrier. $\alpha_{i}$ is the prefactor in SA adsorption isotherm and represents the slope of interfacial tension variation with surface coverage: $\left.\frac{d \gamma}{d \Gamma}\right|_{t=0}$. With time, the relation between $\gamma$ and $\Gamma$ does not probably remain linear. However, for simplicity, we assume a linear relation in this model. We use $\left(\alpha_{2}-\alpha_{1}\right)$ and $\tau$ as the fitting parameters in the calculation of dynamic contact angle profile.

\subsubsection{Comparison of Experiment \& Model}

The dynamics of autophobing drops shows two major features: (i) the timescale for autophobing and (ii) the final contact angle. In our modeling, these features are captured by the two fitting parameters $\tau$ and $\left(\alpha_{2}-\alpha_{1}\right)$, respectively. $\tau$ is inversely proportional to the adsorption rate constant and concentration. In our modeling of the experimental results (see Table 7.1), the found values of $\tau$ are constant irrespective of $\mathrm{CaCl}_{2}$ concentration in the autophobing drop, but vary as the bulk SA concentration changes. This implies that faster autophobing is directly related to the SA concentration 


\begin{tabular}{|l|l|l|l|}
\hline $\mathrm{CaCl}_{2}$ conc. $/$ SA conc. & $\alpha_{2}-\alpha_{1}[\mathrm{~J}]$ & $\tau[\mathrm{s}]$ & $\theta_{f}\left[^{\circ}\right]$ \\
\hline $1 \mathrm{M} / 100 \mu \mathrm{M}$ & $6.37 \times 10^{-21}$ & 30 & 60 \\
\hline $1 \mathrm{M} / 1 \mathrm{mM}$ & $4.77 \times 10^{-21}$ & 10 & 60 \\
\hline $100 \mathrm{mM} / 100 \mu \mathrm{M}$ & $0.95 \times 10^{-21}$ & 30 & 30 \\
\hline
\end{tabular}

Table 7.1: Fit parameters and constants for contact angle evolution model.

( $\left.\tau=\left(c k_{1}\right)^{-1}\right)$, and as SA concentration $c$ increases, $\tau$ decreases leading to a larger slope in the evolution of contact angle. The other fitted parameter $\left(\alpha_{2}-\alpha_{1}\right)$ dictates the magnitude of the final contact angle, and therefore must be related to the amounts of SA adsorbed at the different interfaces. These amounts vary as the $\mathrm{CaCl}_{2}$ concentration changes (as seen from our model parameter values), since $\mathrm{Ca}^{2+}$ acts as the bridging cation and helps SA adsorb to the S/O interface. A higher $\mathrm{CaCl}_{2}$ concentration is thus suggestive of a higher SA adsorption, leading to a higher contact angle. It is important to note that, autophobing only happens when $\alpha_{2}>\alpha_{1}$, which means that at the same adsorption density, the surface pressure must be higher at the $\mathrm{S} / \mathrm{O}$ interface compared to the $\mathrm{O} / \mathrm{W}$ interface.

Figure 7.9 shows the evolution of contact angles with time for autophobing drops under various experimental conditions (dashed lines). The corresponding fits calculated from the model are depicted by the solid lines. In the model, the initial oil-water interfacial tension $\gamma_{0}$ is kept constant at $\gamma_{0}=0.051 \mathrm{~N} / \mathrm{m}$ (from experimental measurements), and the maximum surface density at the oil-water interface $\Gamma_{1}^{\infty}=8 \times 10^{18} / \mathrm{m}^{2}$ [26] is fixed as well. Our model describes the experimental data quite well. Various parameters from the model are summarized in Table 7.1. We note that the prefactor term of the SA adsorption isotherm $\left(\alpha_{2}-\alpha_{1}\right)$ is of the order $\sim k_{B} T$.

\subsubsection{Rate Determining Step}

SA molecules are adsorbed at the $\mathrm{O} / \mathrm{W}$ interface following diffusion from bulk oil. In principle, both diffusion and the interfacial reaction can influence the rate of adsorption at the $\mathrm{O} / \mathrm{W}$ interface. In our model, the local concentration $c$ in the sublayer just outside the drop is assumed to be constant in solving Equation 7.11. In this case, we assume the diffusion to be rapid, implying this subsurface concentration to be very close to the bulk SA concentration $c_{\infty}$. The time-constant $\tau$ can therefore be taken as inversely proportional to $c$, the local surfactant concentration at the subsurface layer. 


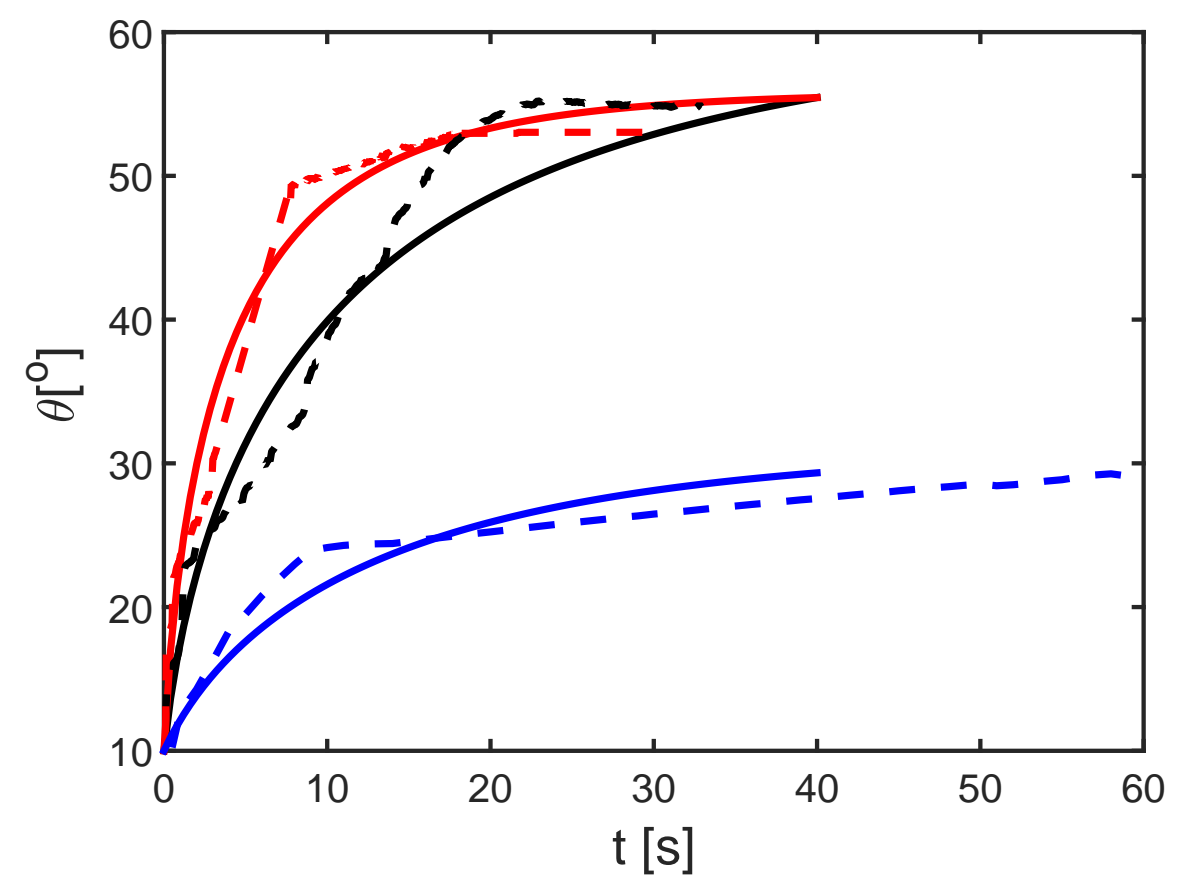

Figure 7.9: Evolution of contact angle vs time, calculated from the model (solid lines) compared with the experimental values (dashed line, average of 15 drops at $\mathrm{pH}$ 7). $\mathrm{CaCl}_{2}$ drops on mica in ambient decane are considered; at two different salt concentrations: $1 \mathrm{M}$ (black) and $100 \mathrm{mM}$ (blue), and two different SA concentration in decane: $100 \mu \mathrm{M}$ (black and blue) and $1 \mathrm{mM}$ (red).

Alternatively, if we assume that diffusion is the rate-limiting step, then the Ward-Tordai equation [27] (corresponding to diffusion to a sink) would be appropriate:

$$
\Gamma=2 c_{\infty} \sqrt{\frac{D t}{\pi}}
$$

with $D$ the diffusion coefficient and $t$ the time after deposition of a fresh drop. Looking at the scaling with $c$ and $t$, this equation predicts that $\frac{\tau_{2}}{\tau_{1}}=$ $\left(\frac{c_{\infty, 1}}{c_{\infty, 2}}\right)^{2}=100$ for $\frac{c_{\infty, 1}}{c_{\infty, 2}}=10$. This is clearly not the case in our experiments, where a change in bulk SA concentration by an order of magnitude (from $100 \mu \mathrm{M}$ to $1 \mathrm{mM}$ ) leads to a time scale change by only $1 / 3$. If we perform the same calculation based on adsorption based Equation $7.15\left(\tau=1 / c k_{1}\right)$, 
we get an expected value of $\frac{\tau_{2}}{\tau_{1}}=\frac{c_{\infty, 1}}{c_{\infty, 2}}=10$. This is closer to our model predictions, although not completely similar. The same order of magnitude for these two time-scales could be taken as an indication that adsorption is indeed the rate-deterministic step in our experiments. However, the occurrence of just one process which is rate limiting under all conditions, is very likely an idealization. Nonetheless, our simple model appears to capture the most important step of the rate-deterministic process.

\subsubsection{Completion of Autophobing}

In our experiments, the autophobing of the aqueous drop stops after a certain time. The time-scale of completion of autophobing depends on the surfactant concentration while the contact angle at this completion is governed by the solute (salt) concentration in the aqueous phase. The precise mechanism via which the autophobing comes to an end, still needs to be elucidated. It might coincide with the formation of a saturated $\mathrm{O} / \mathrm{W}$ interface, which, by definition, means that the adsorption flux has become zero. In our model, the adsorption at $\mathrm{O} / \mathrm{W}$ interface also stops at $\frac{d N}{d t}=0$, which leads to $\dot{A}=0$, meaning the autophobing stops.

Characterization of $\tau$ and $\alpha$ at a certain macroscopic condition (i.e., a combination of $\mathrm{SA}$ and $\mathrm{CaCl}_{2}$ concentrations) is not always straightforward, due to the variability in the time-dependent contact angle. It is important to note that the contact angle evolution trends, as depicted in Figure 7.9, are average trends from our autophobing experiments for specific conditions such as salt concentration or SA concentration. Autophobing drops do not behave in an identical manner even when conditions are identical, as shown in Figure 7.4, which concerns both the time scale and the final contact angle. The variations could be influenced by many factors: such as pinning of the drop while autophobing, small amount of propelling on the solid substrate as the drop undergoes autophobing etc. However, for a significantly large number of droplets, the autophobing behavior is quite general and follows a similar slope and contact angle during evolution. This general behavior was discussed in the current section as the trend characteristic of these drops, and are captured with the model. Capturing the various asymmetry and chemical non-equilibrium issues such as self-propelling or pinning and their subsequent effects on contact angle evolution are quite challenging and have not been addressed in this present model. 


\subsection{Autophobing for Saturated Drops}

Another way to examine our model/hypothesis for the kinetics and termination of autophobing, is to let the aqueous $\mathrm{CaCl}_{2}$ drop age in the fatty acid solution before bringing it in contact with the substrate. This should bring the initial $\mathrm{O} / \mathrm{W}$ interface of the sessile drop closer to the saturated state, possibly leading to an earlier termination of the autophobing. In these experiments, we wait for $10,20,30,60$ or 120 s before the touchdown. Regardless of the waiting time, we found the initial contact angle to be $180^{\circ}$, i.e., the drop does not spread on the solid. However, either by forcing the drop (with the needle) on the substrate or by allowing the drop a few seconds at this $180^{\circ}$ contact angle, the drop can be deposited. The drop then spreads until the contact angle is the same as $\theta_{\min }$, the initial contact angle for non-saturated drops. Remarkably, the drop undergoes autophobing in the same manner as an unsaturated drop: the time-scale and the final equilibrium contact angle remain the same (within the range of the error bars) as in the case of fresh drops, and only depends on the bulk SA concentration in the ambient oil and the salt concentration in the aqueous phase, respectively.

The observation that the aged drops are initially reluctant to spread on the substrate, confirms that the $\mathrm{O} / \mathrm{W}$ interface is indeed covered with SA molecules and the hydrophobic tails of the molecule prevent the aqueous phase to interact with the hydrophillic mica/sillica surface. By forcing the drop on the substrate or by allowing some time, it is apparently possible to remove/disperse this layer of SA molecules. The forcing of the drop will result in the dispersing of the SA molecules if these molecules are not at the interface, but rather a multi-layer of the molecules are formed outside the interface and previous studies have shown that such a multi-layer formation is possible [23]. Another possible explanation might be that the $\mathrm{O} / \mathrm{W}$ interface has bare areas which, if close to the solid substrate, interacts directly with them and forms a wetting layer. A greater volume of the aqueous phase will arrive from the inner part of the drop, resulting in a spreading drop, which then starts accumulating fresh SA molecules in its newly formed $\mathrm{O} / \mathrm{W}$ interface and undergoes autophobing.

The plausible mechanisms underline that the autophobing phenomenon is richer than what our model can capture. Firstly, the model assumes that once the $\mathrm{O} / \mathrm{W}$ interface is saturated with SA molecules, the flux for transferring these molecules onto the solid substrate vanishes, and the autophobing 
stops. But with these aging experiments, we observe that it is possible for saturated interface to get rid of its molecules and start the autophobing process freshly. Secondly, we assume an even distribution of the SA molecules at the $\mathrm{O} / \mathrm{W}$ interface in our model. However, the possible role of bare parts in the $\mathrm{O} / \mathrm{W}$ interface next to a layer of SA molecules, appears to indicate otherwise. Taking these various mechanistic steps into consideration may result in a more robust theoretical model, while one might argue that drop aging is another way to create the same starting point for the model .

\subsection{Concluding Remarks}

We have investigated the adsorption of fatty acid molecules in a three phase system consisting of solid mineral surface, saline aqueous phase and ambient oil phase containing the surfactants. We observe aqueous drops autophobing on the solid substrate, i.e., spontaneously increasing their contact angle manyfold. This phenomenon occurs only when the aqueous phase contains divalent $\mathrm{Ca}^{2+}$ cations and depends on the salt concentration, $\mathrm{pH}$ and the stearic acid concentration in the oil. The final contact angle is influenced by the concentration of divalent cation, while the time-scale of autophobing is governed by the fatty acid concentration. We first established the phenomenon and its reproducibility for a specific system i.e., autophobing on mica in decane with stearic acid, and subsequently showed its general nature by considering various other substrates (silica), oil (heptane and hexadecane) and other fatty acids (hexanoic acid and decanoic acid). The essence of autophobing behaviour remains the same for these various systems, while small differences in contact angle were observed because of fatty acid adsorption depending on the carbon chain length.

We discovered a mechanism where autophobing of a drop occurs without any surfactant in the drop phase. The surfactant in our system gets adsorbed via a chemical reaction at the oil-water interface from the oil, and subsequently, gets deposited on the solid-oil interface leading to autophobing drops. We have validated our proposition using Atomic Force Microscopy, where a large amount of fatty acid is observed next to the contact line of the autophobed drop. There is no evidence of adsorbed fatty acid underneath the drop, ruling out fatty acid transport through the drop and highlighting the fact the adsorption at the oil-water interface is the first step. We developed an adsorption based model in order to capture the evolution of contact 
angle. The model assumes instantaneous equilibrium during the autophobing process, and a Langmuir-Blodgett type adsorption layer on the solid phase. We introduce two fitting parameters in the model, where one represents the relation between interfacial tension and coverage, and the other denotes the time-scale of autophobing. The trend of contact angle evolution in the experiments is captured succesfully with this simplified model, where we have also associated the salt concentration and the fatty acid concentration to our fit parameters in order to represent the major features of the autophobing dynamics. 


\subsection{Appendix I: Autophobing Extended Exper- iments}

\subsubsection{Various Surfactant Concentrations}
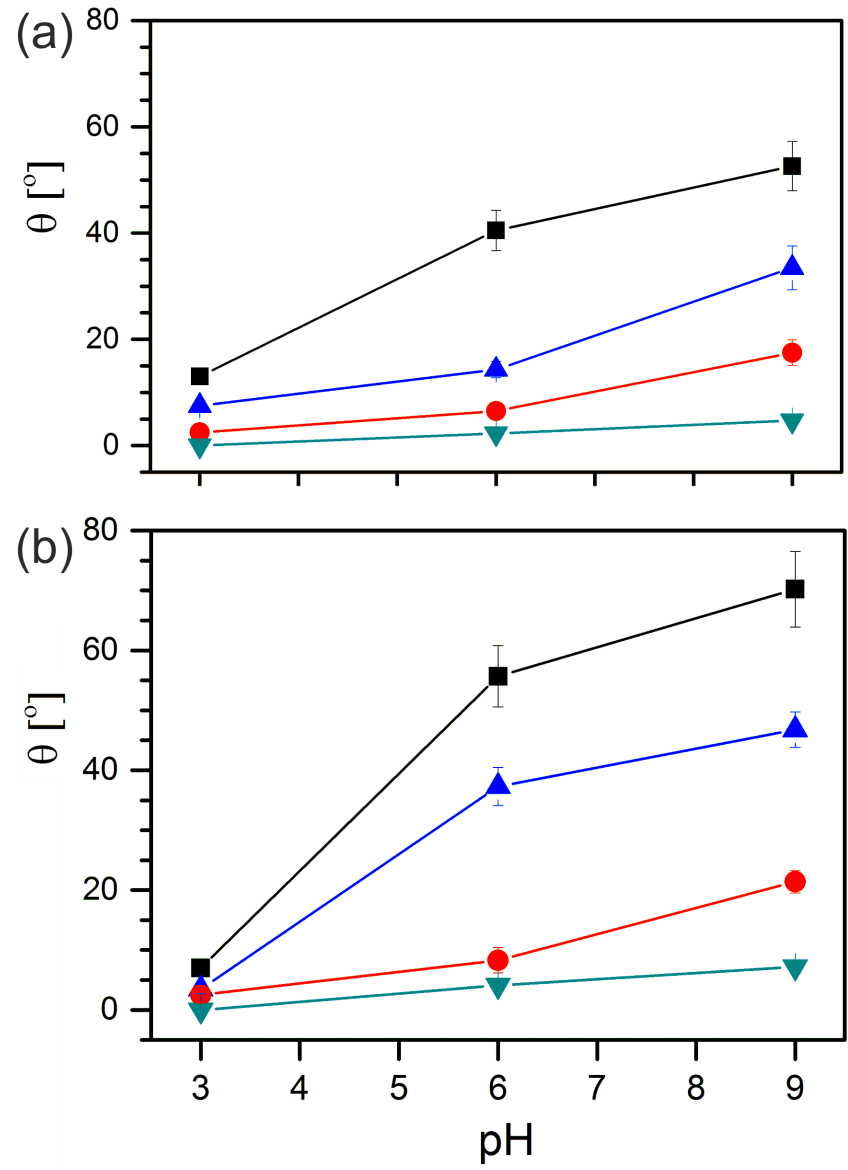

Figure AI.1: Equilibrium contact angles on (a) mica and (b) silica; in ambient decane with stearic acid vs. $\mathrm{pH}$ for various concentrations of $\mathrm{CaCl}_{2}: 1 \mathrm{mM}$ (cyan downward triangles), $10 \mathrm{mM}$ (red circles), $100 \mathrm{mM}$ (blue upward triangles), $1 \mathrm{M}$ (black squares). Stearic acid concentration: $1 \mathrm{mM}$. 


\subsubsection{Influence of Fatty Acid-Chain Length}

In order to investigate the influence of the aliphatic chain length on adsorption, we have performed the autophobing experiments with various fatty acids. As we gradually decrease the aliphatic chain length, a decrease in the final contact angle is observed compared to that for SA. Aqueous drops of $\mathrm{CaCl}_{2}$ in ambient decane containing decanoic acid $\left(\mathrm{CH}_{3}\left(\mathrm{CH}_{2}\right)_{8} \mathrm{COOH}\right)$ autophobes at $\mathrm{pH} 9$ (the autophobing is negligible at $\mathrm{pH}$ 6), and the maximum equilibrium contact angle is $\sim 30^{\circ}$, which is significantly lower than in the case of SA $\left(\sim 60^{\circ}\right)$. If we change the fatty acid from decanoic acid to hexanoic acid $\left(\mathrm{C}_{5} \mathrm{H}_{11} \mathrm{COOH}\right)$ in the ambient decane, then the behaviour of the aqueous drops of $\mathrm{CaCl}_{2}$ are similar to those for decanoic acid, but the equilibrium contact angles are slightly higher.

Hence, as expected, the aliphatic chain length plays a role in the adsorption. It is plausible, that the adsorption of these fatty acid molecules at the $\mathrm{O} / \mathrm{W}$ interface is proportional to the chain length, and hence SA adsorption is strongest, followed by decanoic acid and hexanoic acid. However, the equilibrium contact angle does not follow this order, since the postautophobing contact angle for hexanoic acid lies in between that of decanoic acid and SA. While trying to explain this trend, we have to take into consideration the solubility of these fatty acids. SA and decanoic acid are not soluble in water, while hexanoic acid dissolves in water. This implies, that in the case of hexanoic acid adsorption at $\mathrm{O} / \mathrm{W}$ interface, a fraction of the acid molecules are subsequently transported across the aqueous drop to get directly adsorbed onto the solid substrate. Hence, the substrate is already more hydrophobic (compared to the scenarios of decanoic acid and SA), when the autophobing starts. The contact angle trends for various fatty acids at different $\mathrm{CaCl}_{2}$ concentrations are represented in Figures AI.2.

\subsubsection{Influence of Alkane-Chain Length}

The influence of the aliphatic chain-length of the solvent upon fatty acid adsorption is found out to be relatively small. We have performed experiments switching the solvent of SA from decane to heptane $\left(\mathrm{C}_{7} \mathrm{H}_{16}\right)$ and hexadecane $\left(\mathrm{C}_{16} \mathrm{H}_{34}\right)$. As represented in Figure AI.3, the autophobing trends as well as the equilibrium contact angles are comparable to the case of SA in decane. 

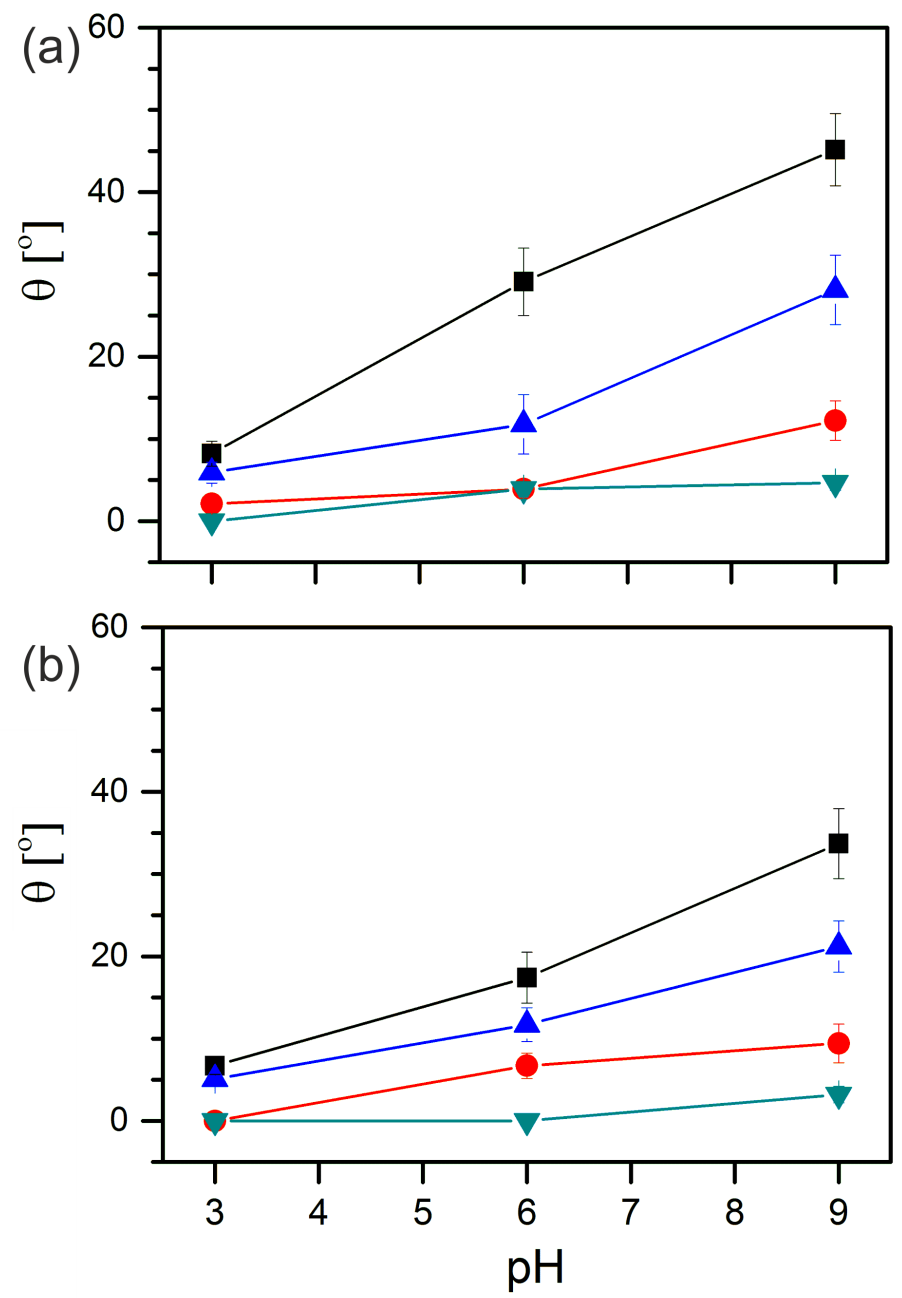

Figure AI.2: Equilibrium contact angles on mica in ambient decane with (a) hexanoic acid and (b) decanoic acid vs. $\mathrm{pH}$ for various concentrations of $\mathrm{CaCl}_{2}$ : $1 \mathrm{mM}$ (cyan downward triangles), $10 \mathrm{mM}$ (red circles), $100 \mathrm{mM}$ (blue upward triangles), $1 \mathrm{M}$ (black squares). Hexanoic and decanoic acid concentration: 100 $\mu \mathrm{M}$. 

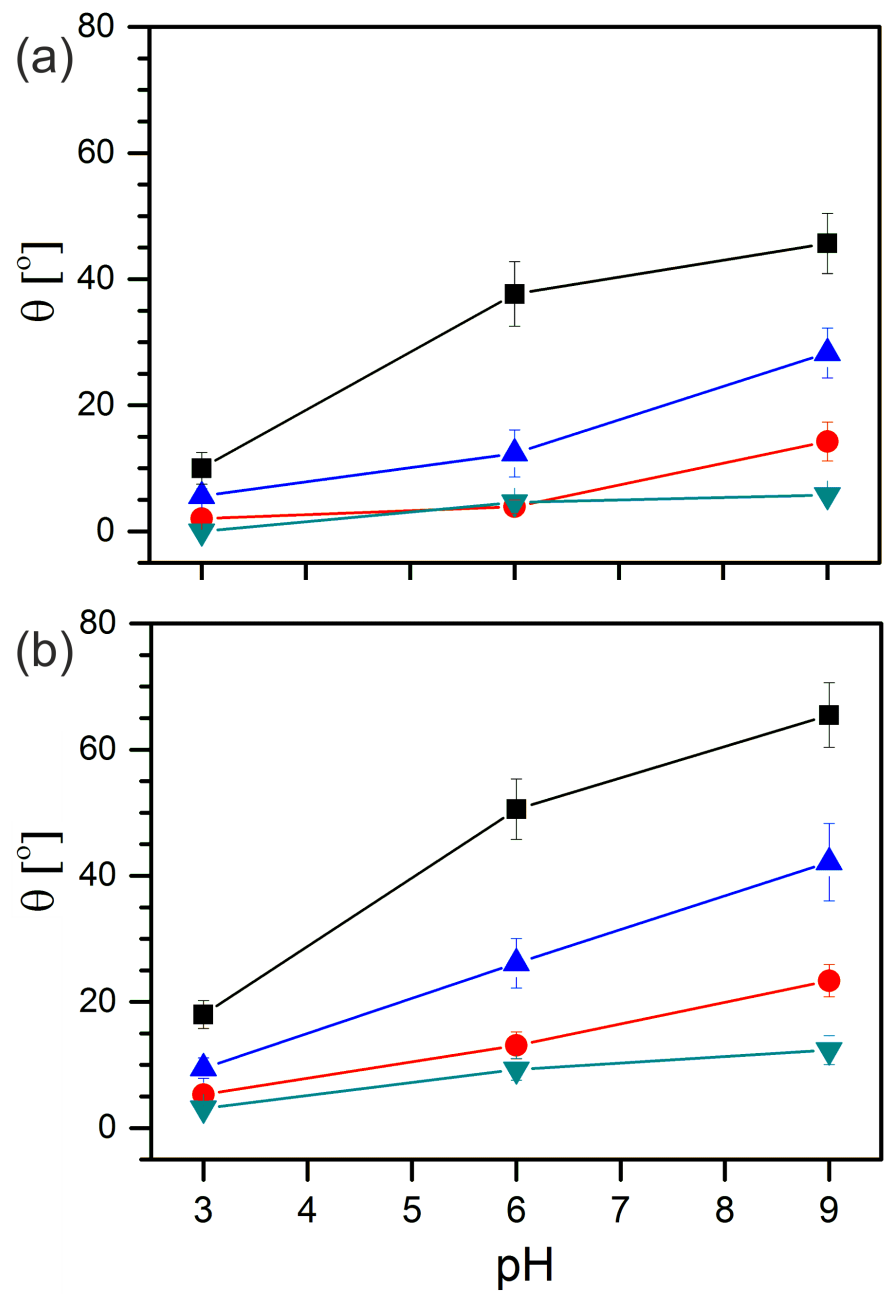

Figure AI.3: Equilibrium contact angles on mica in ambient (a) heptane and (b) hexadecane with stearic acid vs. $\mathrm{pH}$ for various concentrations of $\mathrm{CaCl}_{2}$ : $1 \mathrm{mM}$ (cyan downward triangles), $10 \mathrm{mM}$ (red circles), $100 \mathrm{mM}$ (blue upward triangles), $1 \mathrm{M}$ (black squares). Stearic acid concentration: $1 \mathrm{mM}$. 


\subsubsection{Cationic Surfactant}

Various fatty acid molecules behave as anionic surfactants, since upon deprotonation they yield a negatively charged specie. We have used octadecylamine $\left(\mathrm{CH}_{3}\left(\mathrm{CH}_{2}\right)_{17} \mathrm{NH}_{2}\right)$ as the surface-active component in decane. The molecules are protonated irrespective of the experimental $\mathrm{pH}(3,6$ or 9$)$ and the positively charged species get adsorbed directly to the S/O interface, explaining the large contact angles.
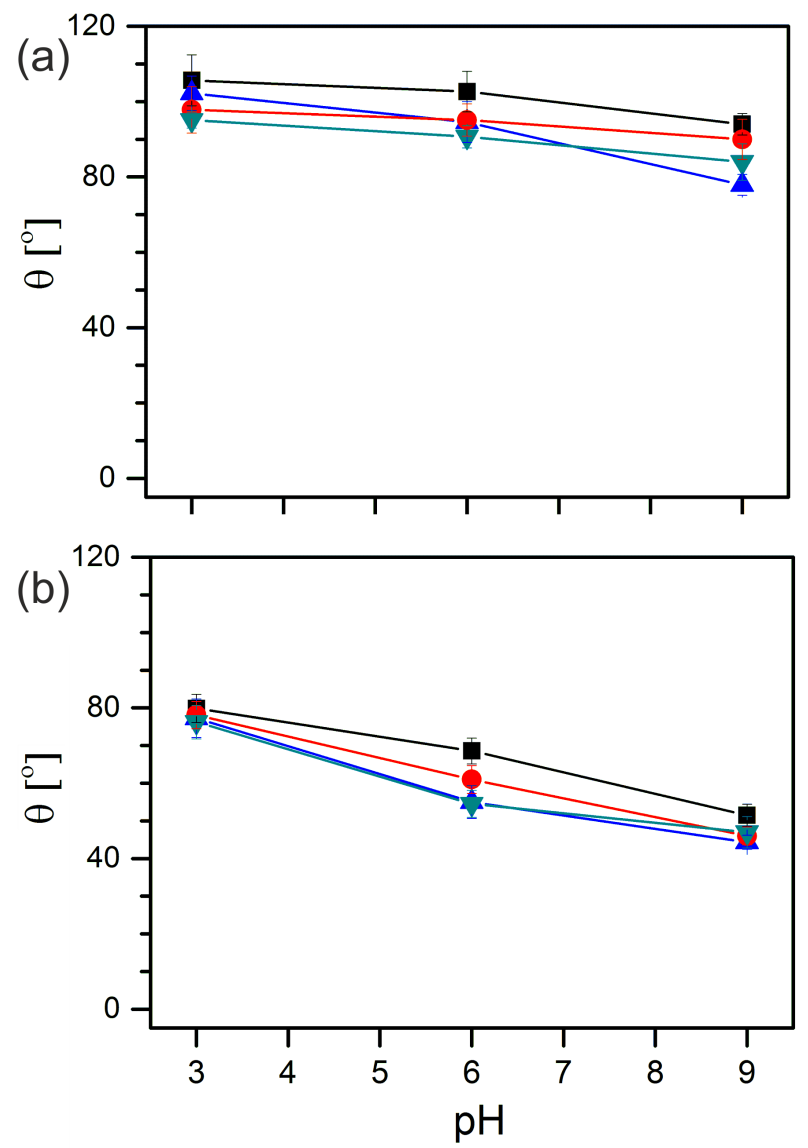

Figure AI.4: Equilibrium contact angles in ambient decane with octadecylamine on (a) mica and (b) silica vs. $\mathrm{pH}$ for various concentrations of $\mathrm{CaCl}_{2}$ : $1 \mathrm{mM}$ (cyan downward triangles), $10 \mathrm{mM}$ (red circles), $100 \mathrm{mM}$ (blue upward triangles), $1 \mathrm{M}$ (black squares). Octadecyl Amine concentration: $100 \mu \mathrm{M}$. 


\subsection{Appendix II: Calculation for Autophob- ing Modeling}

In section 7.5, we have introduced a model to address the salient features of adsorption kinetics and evolution of contact angle during autophobing. However, the mechanism of autophobing contains several concurrent processes and we have used a number of assumptions in the described model to simplify the analysis. In the following section of Appendix, we will provide the general approach of this modeling, which is valid under all conditions.

The aqeuous drop is considered to be of spherical cap geometry during the entire process of autophobing, hence the radius of the drop as well as the radius of the contact line is a function of contact angle $\theta$ through the following relations:

$$
\begin{aligned}
R & =R_{0}\left(\frac{4}{2-3 \cos \theta+\cos ^{3} \theta}\right)^{1 / 3} \\
r & =R \sin \theta \\
A & =2 \pi R^{2}(1-\cos \theta) \\
L & =2 \pi R \sin \theta
\end{aligned}
$$

where $R$ is the radius of the spherical cap and $R_{0}=(3 V /(4 \pi))^{1 / 3}$.

The contact angle of the autophobing drop at a specific time-instant $t$ is assumed to be the result of an instantaneous equilibrium, where both the $\mathrm{O} / \mathrm{W}$ and the $\mathrm{S} / \mathrm{O}$ interfacial tension decrease over time due to a progressive accumulation of surface active material leading to higher densities. Here, the first step is the formation of a surfactant layer at the oil-water interface (Figure AII.1a, left) from the ambient solution by diffusion followed by adsorption. The transfer of SA molecules to the solid substrate starts as soon as the surfactant layer forms at the $\mathrm{O} / \mathrm{W}$ interface, and this transfer is considered to occur along the oil-water contact surface i.e., surface of the spherical cap. We define $N_{1}$ as the number of molecules adsorbed in the O/W interface while $N_{2}$ is the number of molecules adsorbed on the substrate (from the $\mathrm{O} / \mathrm{W}$ interface) in a rim of width $w$ just outside the contact line. This width is assumed to be of the order of the size of an SA molecule. The area of the $\mathrm{O} / \mathrm{W}$ interface is $A$ and the length of the contact line is $L=2 \pi r$ where 

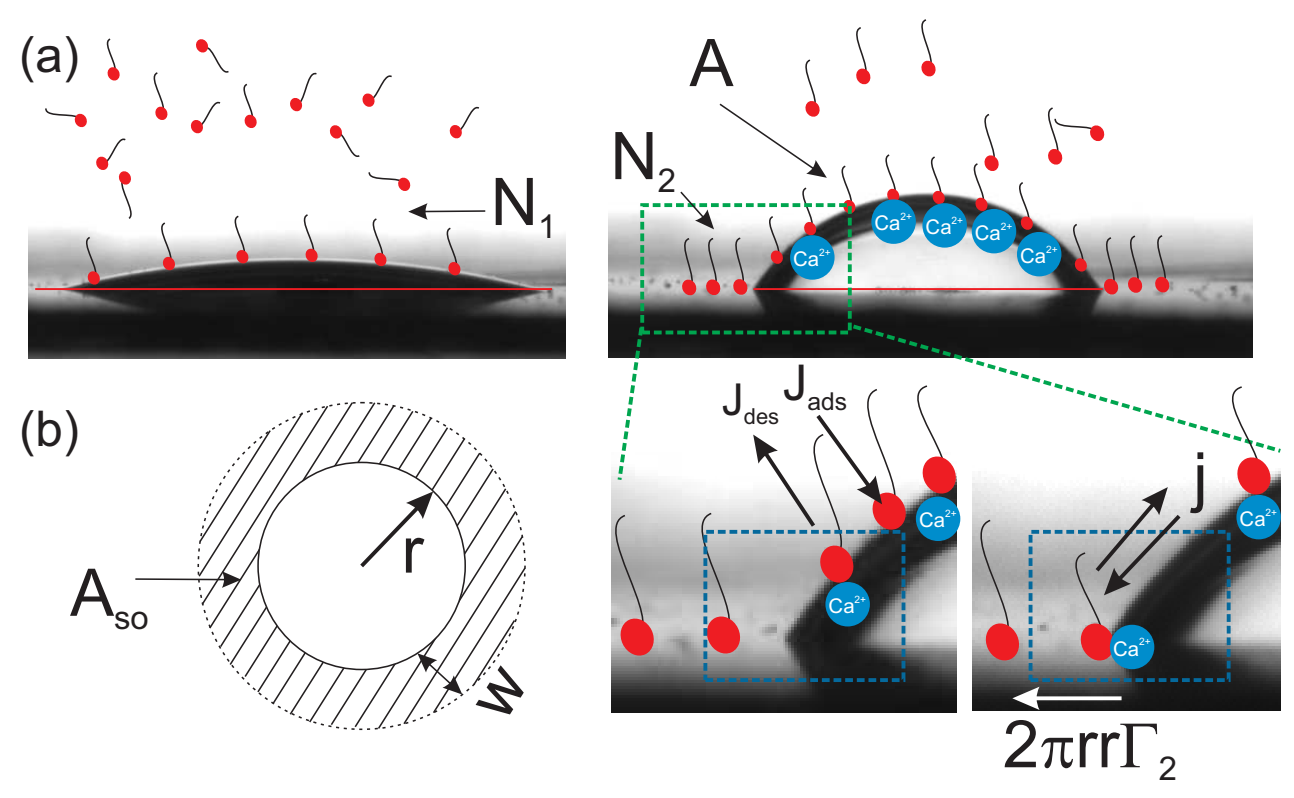

(b)

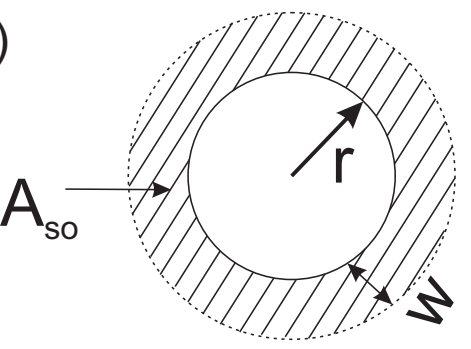

Figure AII.1: Stearic acid adsorption occurs first from bulk ambient alkane phase to the oil-water interface (a, left) and then subsequently the molecules get deposited on the solid substrate (a,right). The enlarged depiction of this deposition process is shown on $b$, right. (b, left) stearic acid coverage (hatched area) on a solid substrate for an autophobing drop. The intitial three-phase contact line is depicted by the dashed line, while the receding contact line is shown by the solid line.

$r$ is the radius of the circular contact line. Furthermore, we define the surface densities $\Gamma_{1}=N_{1} / A$ and $\Gamma_{2}=N_{2} /(w L)$. The autophobing drops are considered to be of sphreical cap geometry where the contact angle $\theta$ is supposed to increase from a minimum value $\theta_{\min }$ to a maximum value $\theta_{\max } \approx 60^{\circ}$ after the autophobing. Following the arguments presented in the main text, we can write the instantaneous contact angle in form of the Young's equation:

$$
\cos \theta(t)=\frac{\gamma_{s o}(t)-\gamma_{s w}}{\gamma_{o w}(t)}
$$

We consider the autophobing a quasi-ideal process, where the interfacial tension $\gamma$ and the coverage density $\Gamma$ linearly related: $\gamma_{o w}=\gamma_{o w, 0}-\alpha_{1} \Gamma_{1}$ and $\gamma_{s o}=\gamma_{s o, 0}-\alpha_{2} \Gamma_{2}$. Hence, we can write: 


$$
\begin{aligned}
& \text { or, } \cos \theta(t)=\frac{\gamma_{s o, 0}-\alpha_{2} \Gamma_{2}-\gamma_{s w}}{\gamma_{o w, 0}-\alpha_{1} \Gamma_{1}} \\
& \text { or, } \cos \theta(t)=\frac{\gamma_{0} \cos \theta_{0}-\alpha_{2} \Gamma_{2}(t)}{\gamma_{0}-\alpha_{1} \Gamma_{1}(t)}
\end{aligned}
$$

We can now formulate the following rate equations:

$$
\begin{aligned}
\frac{d N_{1}}{d t} & =A\left(J_{\mathrm{ads}}-J_{\mathrm{des}}\right)-L j \\
\frac{d N_{2}}{d t} & =L j+\dot{r} L \Gamma_{2}
\end{aligned}
$$

where, $J_{a d s}$ and $J_{\text {des }}$ are the adsorption and desorption fluxes of SA from the bulk oil phase to the $\mathrm{O} / \mathrm{W}$ interface, respectively, and $j$ is the outward SA flux along the contact line for deposition from $\mathrm{O} / \mathrm{W}$ to the $\mathrm{S} / \mathrm{O}$ interface. The term $\dot{r} L \Gamma_{2}$ represents the molecules that leave the contact line area as the drop autophobes because they stick to the substrate and do not move with the contact line inwards. The fluxes are defined by:

$$
\begin{aligned}
J_{\mathrm{ads}} & =c k_{1}\left(\Gamma_{\infty}-\Gamma_{1}\right) \\
J_{\mathrm{des}} & =k_{2} \Gamma_{1} \\
j & =k_{3} \Gamma_{1}-k_{4} \Gamma_{2}
\end{aligned}
$$

where $c$ is the concentration of the molecules in the oil phase near the wateroil interface and $k_{n}$ are rate constants.

In order to solve Eqs. (7.24) and (7.25), a relation needs to be established between $\theta$ and $\Gamma_{1}$ and $\Gamma_{2}$. We define the function $\mathrm{F}$ as:

$$
F\left(\Gamma_{1}, \Gamma_{2}, \theta\right)=\cos \theta-\frac{\gamma_{0} \cos \theta_{0}-\alpha_{2} \Gamma_{2}}{\gamma_{0}-\alpha_{1} \Gamma_{1}}=0
$$

From this expression we observe that autophobing $\left(\cos \theta<\cos \theta_{0}\right)$ is only possible if $\alpha_{2} \Gamma_{2}>\alpha_{1} \Gamma_{1} \cos \theta_{0}$. Using Equation (7.29) we can relate $\dot{\theta}$ with $d N_{1} / d t$ and $d N_{2} / d t$ :

$$
\frac{\partial F}{\partial \theta} \dot{\theta}+\frac{\partial F}{\partial \Gamma_{1}} \frac{d \Gamma_{1}}{d t}+\frac{\partial F}{\partial \Gamma_{2}} \frac{d \Gamma_{2}}{d t}=0
$$

or:

$$
\dot{\theta}=C_{1} \frac{d \Gamma_{1}}{d t}+C_{2} \frac{d \Gamma_{2}}{d t}
$$


where,

$$
C_{1}\left(\Gamma_{1}, \Gamma_{2}, \theta\right)=\left(\frac{\partial F}{\partial \Gamma_{1}}\right)\left(-\frac{\partial F}{\partial \theta}\right)^{-1} \quad C_{2}\left(\Gamma_{1}, \Gamma_{2}, \theta\right)=\left(\frac{\partial F}{\partial \Gamma_{2}}\right)\left(-\frac{\partial F}{\partial \theta}\right)^{-1}
$$

Finally $\dot{A}$ and the velocity of the contact line $\dot{r}$ are given by:

$$
\dot{A}=\frac{d A}{d \theta} \dot{\theta} \quad \text { and } \quad \dot{r}=\frac{d r}{d \theta} \dot{\theta}
$$

We can replace $N_{1}=A \Gamma_{1}$ in the rate equation 7.24:

$$
\begin{aligned}
& \frac{d\left(\Gamma_{1} A\right)}{d t}=A c k_{1}\left(\Gamma_{\infty}-\Gamma_{1}\right)-A k_{2} \Gamma_{1}-L\left(k_{3} \Gamma_{1}-k_{4} \Gamma_{2}\right) \\
& o r, \frac{d \Gamma_{1}}{d t}=\left[c k_{1}\left(\Gamma_{\infty}-\Gamma_{1}\right)-k_{2} \Gamma_{1}-\frac{L}{A}\left(k_{3} \Gamma_{1}-k_{4} \Gamma_{2}\right)\right]-\frac{\frac{d A}{d t}}{A} \Gamma_{1}
\end{aligned}
$$

Similarly, from Equation 7.25, we obtain:

$$
\frac{d \Gamma_{2}}{d t}=\frac{k_{3} \Gamma_{1}-k_{4} \Gamma_{2}}{w}+\frac{\dot{r} \Gamma_{2}}{w}-\frac{\Gamma_{2}}{L} \frac{d L}{d t}
$$

Summarizing the results so far:

$$
\begin{aligned}
& \frac{d \Gamma_{1}}{d t}=B_{1}-\frac{A^{\prime}}{A} \Gamma_{1}\left(C_{1} \frac{d \Gamma_{1}}{d t}+C_{2} \frac{d \Gamma_{2}}{d t}\right) \\
& \frac{d \Gamma_{2}}{d t}=B_{2}-\frac{(w-r) r^{\prime}}{w r} \Gamma_{2}\left(C_{1} \frac{d \Gamma_{1}}{d t}+C_{2} \frac{d \Gamma_{2}}{d t}\right)
\end{aligned}
$$

where we defined:

$$
\begin{aligned}
B_{1}\left(\Gamma_{1}, \Gamma_{2}, \theta\right) & =c k_{1} \Gamma_{1}^{\infty}-\left(c k_{1}+k_{2}\right) \Gamma_{1}-L / A\left(k_{3} \Gamma_{1}-k_{4} \Gamma_{2}\right) \\
B_{2}\left(\Gamma_{1}, \Gamma_{2}, \theta\right) & =k_{3} \Gamma_{1} / w-k_{4} \Gamma_{2} / w \\
A^{\prime} & =\frac{d A}{d \theta} \quad \text { and } \quad r^{\prime}=\frac{d r}{d \theta}
\end{aligned}
$$

Rearranging the terms in Equation (7.37) and (7.38) gives:

$$
\left(\begin{array}{ll}
M_{11} & M_{12} \\
M_{21} & M_{22}
\end{array}\right)\left(\begin{array}{l}
\dot{\Gamma}_{1} \\
\dot{\Gamma}_{2}
\end{array}\right)=\left(\begin{array}{c}
B_{1} \\
B_{2}
\end{array}\right)
$$

with

$$
\left(\begin{array}{ll}
M_{11} & M_{12} \\
M_{21} & M_{22}
\end{array}\right)=\left(\begin{array}{ll}
1+\Gamma_{1} C_{1} A^{\prime} / A & \Gamma_{1} C_{2} A^{\prime} / A \\
\Gamma_{2} C_{1}(w-r) r^{\prime} /(w r) & 1+\Gamma_{2} C_{2}(w-r) r^{\prime} /(w r)
\end{array}\right)
$$


Inversion of Equation 7.42 results in:

$$
\left(\begin{array}{c}
\dot{\Gamma}_{1} \\
\dot{\Gamma}_{2}
\end{array}\right)=\frac{1}{[M]}\left(\begin{array}{cc}
M_{22} & -M_{12} \\
-M_{21} & M_{11}
\end{array}\right)\left(\begin{array}{c}
B_{1} \\
B_{2}
\end{array}\right)
$$

where $[M]=M_{11} M_{22}-M_{12} M_{21}$ is the determinant of the matrix $\{M\}$. If we have values for the parameters $k_{n}$ and $\alpha_{n}$, we can use Equation 7.44 to solve $\Gamma_{1}, \Gamma_{2}$ and $\theta$ as a function of time. 


\subsection{Appendix III: Wettability Alteration on Langmuir-Blodgett Layers}

In this chapter, autophobing experiments were presented where SA dissolved in ambient oil phase gets adsorbed at oil-water interface (in presence of $\mathrm{CaCl}_{2}$ in the aqueous phase) and subsequently gets deposited on bare substrate to form a layer. In reality, some examples such as oil recovery involve the adsorption of polar components on solid phase prior to any contact with oil. Hence, the oil-water competitive wettability only comes into play, when the substrate already contains a previously formed layer of organic component. We report in this section, the results of oil-water relative wetting on substrates where a SA layer is grown using Langmuir-Blodgett (LB) transfer method prior to the wetting experiments. Recent investigation [25] has focussed on stability of such layers based on aqueous composition in the wetting drop, but studying the wetting properties in ambient oil is unexplored.

\subsubsection{Chemicals and Materials}

Octadecanoic acid i.e., stearic acid (Sigma Aldrich, grade 1, 98\%) solution is prepared in $\mathrm{CHCl}_{3}$ (Sigma Aldrich, ACS reagent grade) at $1 \mathrm{mg} / \mathrm{ml}$ concentration. The subphase for the organic layer deposition for all the experiments is $0.01 \mathrm{M} \mathrm{CaCl}_{2}$ solution. The preparation of the subphase as well as various aqueous salt solutions for contact angle goniometry are done in the similar manner to that described in section 7.2. The mixture of various salt solutions consists of $10 \mathrm{mM} \mathrm{CaCl}_{2}, 50 \mathrm{mM} \mathrm{MgCl}_{2}$ and $500 \mathrm{mM} \mathrm{NaCl}$ in order to partially mimic the brine (Artificial Sea Water, ASW) solution used in oil-recovery. We will use the term 'semi-Artificial SeaWater (semi-ASW)' in order to describe the various experiments with this mixture solution.

The organic layer deposition is carried out on silica surfaces. Silica substrates of dimension $4 \mathrm{~cm} \times 1 \mathrm{~cm}$ are cut from commercial silicon (100) wafers with a thermally grown oxide layer of $(\sim 35 \mathrm{~nm})$ thickness. The substrates are extensively cleaned prior to the deposition, first in a mixture of ethanol, isopropanol and Millipore water and left in an ultrasound sonication bath for 5 minutes. Then the substrates are removed from the bath, rinsed with Millipore water and dried carefully with $\mathrm{N}_{2}$ gas. Subsequently, the wafers were treated with ambient air plasma (PDC-32G-2 Har- 
rick Plasma) for at least 30 min which assures the hydrophilicity of the substrate. The contact angle of pure water on these substrates is observed to be less than $5^{\circ}$ in air.

\subsubsection{Langmuir-Blodgett Method}

The organic layer on substrates is prepared using an automated Langmuir Blodgett trough (NIMA technology, model 1212D1). The details of the trough and the deposition method are discussed in Chapter 3.

\subsubsection{Dynamic Contact Angle Behavior}

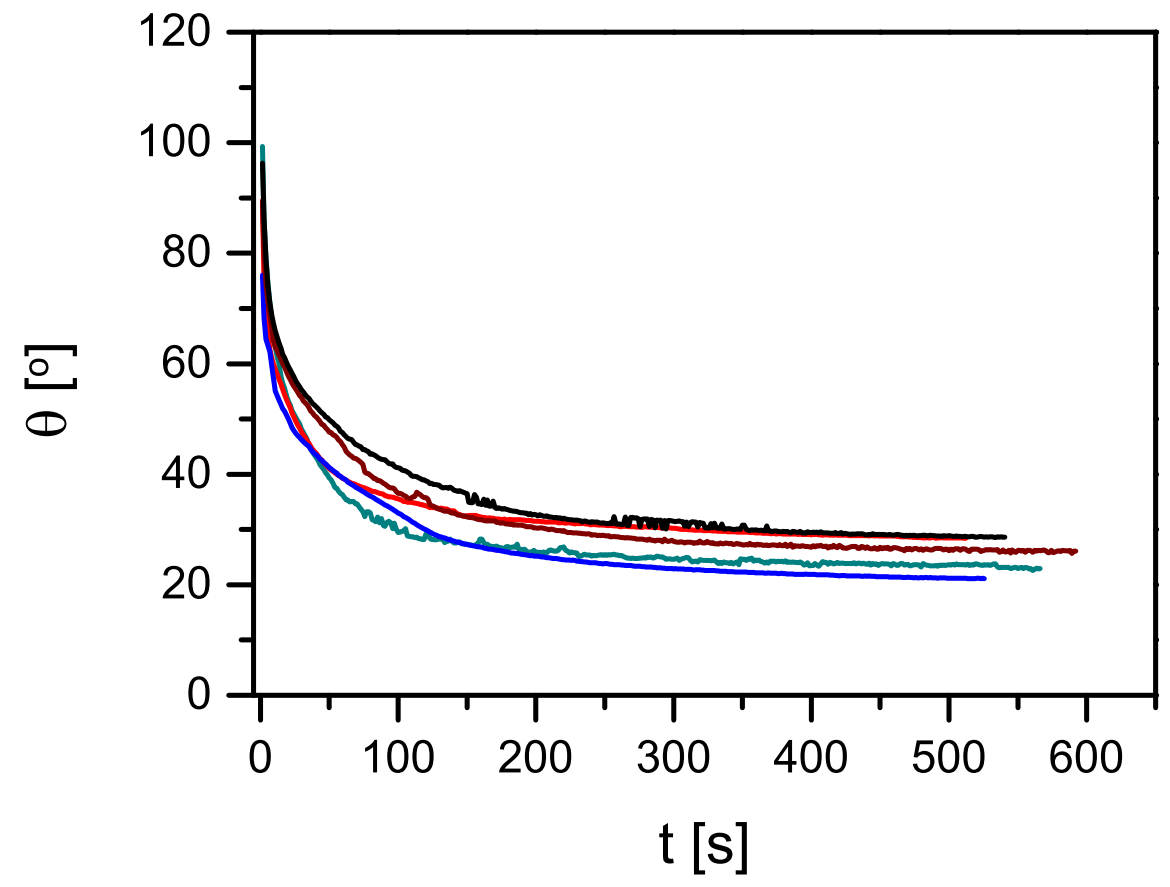

Figure AIII.1: Contact angle vs time for aqueous drops on silica substrates with LB monolayer of SA in ambient decane; aqueous drops containing $\mathrm{CaCl}_{2}$ at various concentrations: $1 \mathrm{M}$ (black), $500 \mathrm{mM}$ (brown), $100 \mathrm{mM}$ (blue), $10 \mathrm{mM}$ (red) and $1 \mathrm{mM}$ (dark cyan). The contact angle evolution trend is average of 10 individual drops under identical conditions. 
Figure AIII.1 shows the contact angle change w.r.t time for aqueous drops containing $\mathrm{CaCl}_{2}$ at various concentrations between $1 \mathrm{mM}$ and $1 \mathrm{M}$. Upon placing these drops on silica substrates with a layer of SA (deposited by LB trough), the initial contact angle is $\sim 100^{\circ}$, which is expected on a hydrophobic surface. The contact angles gradually decrease over time with a final contact angle of $\sim 25-35^{\circ}$ in about $10 \mathrm{~min}$. The final contact angle is observed to be independent of $\mathrm{CaCl}_{2}$ concentration.

Figure AIII.2 shows contact angle evolution over time for drops of semiASW (black). The contact angle drop is similar to that of $\mathrm{CaCl}_{2}$ drops ( $100^{\circ}$ to $\left.30^{\circ}\right)$. Diluting the semi-ASW $(10 \%$, red line) does not have an effect on the change of contact angle.

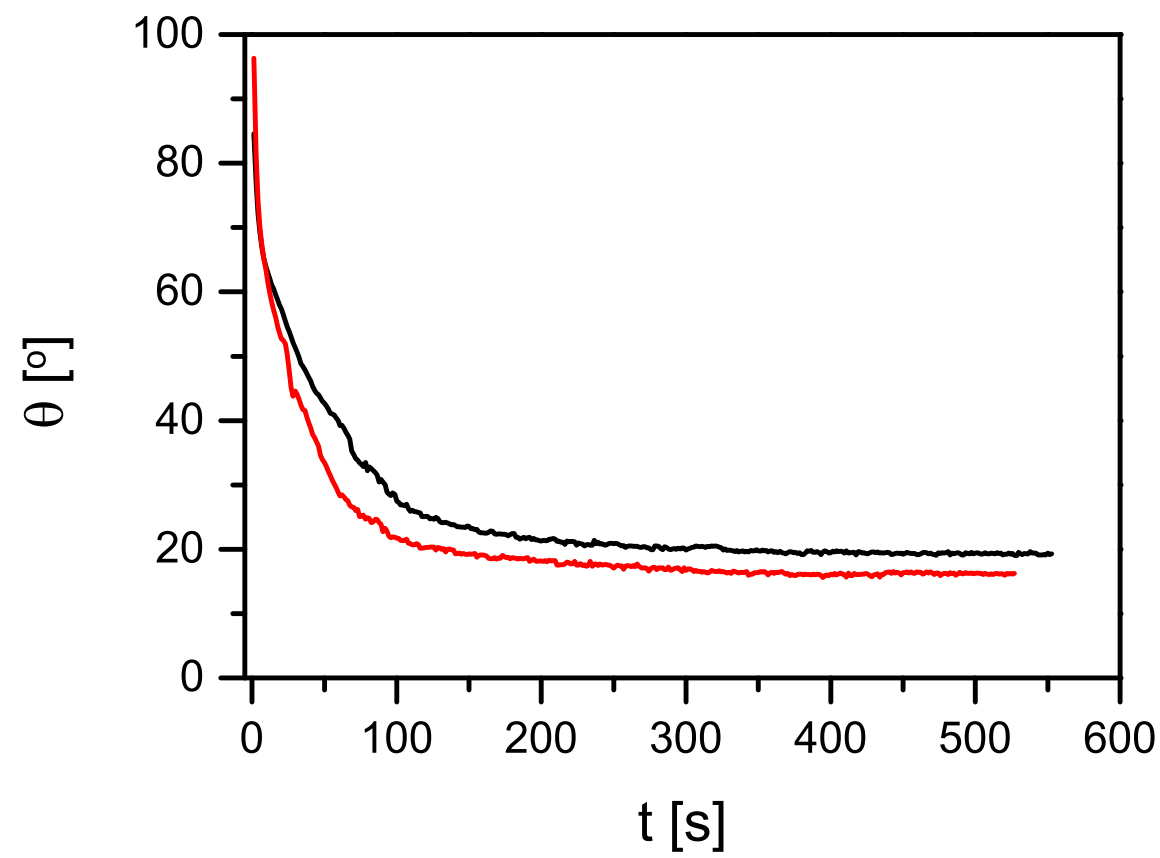

Figure AIII.2: Contact angle vs time for aqueous drops on silica substrates with LB monolayer of SA in ambient decane; aqueous drops containing composition of Semi-ASW (black; $10 \mathrm{mM} \mathrm{CaCl}_{2}, 50 \mathrm{mM} \mathrm{MgCl}_{2}$ and $500 \mathrm{mM} \mathrm{NaCl}$ ) and $10 \%$ Semi-ASW (red). The contact angle evolution trend is average of 10 individual drops under identical conditions.

The mechanism of contact angle decrease is governed by the desorption of SA molecules from the silica substrate. Our experiments show that the 
ability of the aqueous drop to desorb these SA molecules does not depend on the $\mathrm{CaCl}_{2}$ concentration of the drop. We have also investigated the state of the SA layer after the wetting experiments, where we observe that the desorbed layer is similar after the wetting experiments with aqueous drops of various $\mathrm{CaCl}_{2}$ concentration. This highlights the fact that recreating a complex reservoir rock surface with its various polar molecules, competing adsorption/desorption processes is quite challenging and a classic LB transfer is probably not sufficient in order to do that. Furthermore, it also highlights the fundamental difference between an autophobing experiment and a spreading experiment. In autophobing experiment a layer of SA is spontaneously formed on the substrate aided by SA adsorption at oil-water interface. For the LB transfer, however, we create the SA layer using pressure and subsequently desorb the layer using aqueous salt solution. These adsorption and desorption processes are not similar, resulting in the differences in salt-dependent behavior. 


\section{Bibliography}

[1] A. B. Theberge, F. Courtois, Y. Schaerli, M. Fischlechner, C. Abell, F. Hollfelder, and W.T.S. Huck. Microdroplets in microfluidics: An evolving platform for discoveries in chemistry and biology. Angewandte Chemie International Edition, 49(34):5846-5868, 2010.

[2] R. Seemann, M. Brinkmann, T. Pfohl, and S. Herminghaus. Droplet based microfluidics. Reports on Progress in Physics, 75(1):016601, 2012.

[3] F. Brochard. Motions of droplets on solid surfaces induced by chemical or thermal gradients. Langmuir, 5(2):432-438, 1989.

[4] F. Mugele and J.C. Baret. Electrowetting: from basics to applications. Journal of Physics: Condensed Matter, 17(28):R705, 2005.

[5] R. de Ruiter, A. M. Pit, V. M. de Oliveira, M. H. G. Duits, D. van den Ende, and F. Mugele. Electrostatic potential wells for on-demand drop manipulation in microchannels. Lab on a Chip, 14(5):883-891, 2014.

[6] K. D. Barton and R. Shankar Subramanian. The migration of liquid drops in a vertical temperature gradient. Journal of Colloid and Interface Science, 133(1):211-222, 1989.

[7] A.W. Adamson. Physical Chemistry of Surfaces. Wiley, New York, 3 edition, 1976.

[8] K. Lum, D. Chandler, and J. D. Weeks. Hydrophobicity at small and large length scales. The Journal of Physical Chemistry B, 103(22):4570$4577,1999$.

[9] B. J. Berne, J. D. Weeks, and R. Zhou. Dewetting and hydrophobic interaction in physical and biological systems. Annual Review of Physical Chemistry, 60(1):85-103, 2009. 
[10] A. B. Afsar-Siddiqui, P. F. Luckham, and O. K. Matar. The spreading of surfactant solutions on thin liquid films. Advances in Colloid and Interface Science, 106:183-236, 2003.

[11] K. John, M. Bär, and U. Thiele. Self-propelled running droplets on solid substrates driven by chemical reactions. Eur. Phys. J. E, 18(2):183-199, 2005.

[12] U. Thiele, K. John, and M. Bär. Dynamical model for chemically driven running droplets. Physical Review Letters, 93(2):027802, 2004.

[13] H. W. Fox and W. A. Zisman. The spreading of liquids on low energy surfaces.1. Polytetrafluoroethylene. Journal of Colloid Science, 5(6):514$531,1950$.

[14] H. W. Fox and W. A. Zisman. The spreading of liquids on low-energy surfaces.3. Hydrocarbon surfaces. Journal of Colloid Science, 7(4):428442, 1952.

[15] E. F. Hare and W. A. Zisman. Autophobic liquids and the properties of their adsorbed films. Journal of Physical Chemistry, 59(4):335-340, 1955.

[16] H.M. Princen, A.M. Cazabat, M.A. Cohen Stuart, F. Heslot, and S. Nicolet. Instabilities during wetting processes: Wetting by tensioactive liquids. Journal of Colloid and Interface Science, 126(1):84 - 92, 1988.

[17] R. Sharma, R. Kalita, E. R. Swanson, T. E. Corcoran, S. Garoff, T. M. Przybycien, and R. D. Tilton. Autophobing on liquid subphases driven by the interfacial transport of amphiphilic molecules. Langmuir, 28(43):15212-15221, 2012.

[18] N. Kumar, K. Varanasi, R. D. Tilton, and S. Garoff. Surfactant selfassembly ahead of the contact line on a hydrophobic surface and its implications for wetting. Langmuir, 19(13):5366-5373, 2003.

[19] B. Frank and S. Garoff. Surfactant self-assembly near contact lines: Control of advancing surfactant solutions. Colloids and Surfaces aPhysicochemical and Engineering Aspects, 116(1-2):31-42, 1996.

[20] R. V. Craster and O. K. Matar. On autophobing in surfactant-driven thin films. Langmuir, 23(5):2588-2601, 2007.

[21] R. V. Craster and O. K. Matar. Dynamics and stability of thin liquid films. Reviews of Modern Physics, 81(3):1131, 2009. 
[22] B. Frank and S. Garoff. Temporal and spatial development of surfactant self-assemblies controlling spreading of surfactant solutions. Langmuir, 11(11):4333-4340, 1995.

[23] R. de Ruiter, R. W. Tjerkstra, M. H. G. Duits, and F. Mugele. Influence of cationic composition and ph on the formation of metal stearates at oil-water interfaces. Langmuir, 27(14):8738-8747, 2011.

[24] A. M. Brzozowska, F. Mugele, and M. H. G. Duits. Stability and interactions in mixed monolayers of fatty acid derivatives on artificial sea water. Colloids and Surfaces A: Physicochemical and Engineering Aspects, 433:200-211, 2013.

[25] N. Kumar, L. Wang, I. Siretanu, M. H. G. Duits, and F. Mugele. Salt dependent stability of stearic acid langmuir-blodgett films exposed to aqueous electrolytes. Langmuir, 29(17):5150-5159, 2013.

[26] J. Luetzenkirchen, T. Preocanin, and N. Kallay. A macroscopic water structure based model for describing charging phenomena at inert hydrophobic surfaces in aqueous electrolyte solutions. Phy. Chem. Chem. Phys., 10:4946, 2008.

[27] A. F. H. Ward and L. Tordai. Time dependence of boundary tensions of solutions i. the role of diffusion in time effects. The Journal of Chemical Physics, 14(7):453-461, 1946. 


\section{CHAPTER 8}

\section{Conclusions and Outlook}

\subsection{Thesis Contributions}

In this thesis, we investigated the aqueous wettability of mineral surfaces in ambient alkanes influenced by the adsorption of ionic species present in the aqueous phase or polar organic acids present in the alkane. In the following section, we present the highlights of our observations and theoretical analysis.

We have observed a wetting alteration on mica surfaces from unperceivably small $\left(\theta<2^{\circ}\right)$ to finite contact angles as we switch from a monovalent to a divalent (cationic) salt solution, at high salt concentration and $\mathrm{pH}$. In other words, as we change the cation-valency (from a monovalent to a divalent cation), a significant effect on adsorption and wetting on mica is demonstrated in Chapter 4. The origin of this phenomenon is identified as the competitive adsorption of cations at the mica-water interface, where adsorption of divalent cation leads to a charge reversal on the mica surface. The oil-water interface is always negatively charged, and hence positive charge at the mica-water interface (after charge reversal) causes an attractive disjoining pressure in the wetting film. Ellipsometry confirms a 'thick' to 'thin' transition in the wetting film, and surface charges at mineral-water and oil-water interfaces are investigated thoroughly using a streaming potential method.

In order to investigate the electrostatic interactions between the adsorbed molecules and interfaces, we addressed charge-reversal from a theoretical perspective. A surface complexation model is introduced for adsorbing species at the interfaces, and subsequently coupled with a mean-field Poisson Boltzmann approach. The numerical solution using constant charge 
boundary conditions shows that charge-reversal occurs at the mica surface in the presence of divalent cations at a relatively high salt concentration. This corroborates our experimental observations of wettability alteration at a high divalent salt concentration. Subsequently, we presented a linear approach to the electrostatics problem in order to investigate the phenomenon analytically. Charge regulation boundary conditions are used to emulate the variable nature of surface charge. With this model, we have described the various wetting configurations resulting from the electrostatic interactions, and the importance of choosing the right boundary conditions. It is shown that the essence of the model can be captured with two parameters, which govern a transition from near-zero to a finite contact angle in our system. The charge-reversal effect in the presence of multivalent cations is well-known, but we have built a systematic structure of experiments and modelling, in order to relate adsorption, electrostatics and macroscopic wetting.

Molecular interactions are diverse in nature, and involve many effects other than electrostatic. Our subsequent attempt at understanding adsorption and wetting has addressed mica wetting in alkanes in the presence of various alkali metal ions and earth alkali metal ions. We observed that a change in wetting configuration is not exclusively the result of a change in cation-valency. As cations of various size but the same valency are used, the effects of van der Waals, hydration, dispersion interactions play a major role in adsorption, interaction between interfaces and wetting. The knowledge of ion-specific adsorption is more than a century old, but our approach combines the picture with a macroscopic wetting perspective, and for the first time, we introduce a cationic Hofmeister series for mineral wettability in oil.

The last phase of the thesis addresses the rich behavior of amphiphilic polar molecules ubiquitous in relevant systems. In the presence of a fatty acid in the ambient oil phase, and a divalent cation in the aqueous phase, a significant change in drop wettability on mineral substrates occurs. We observe autophobing, i.e., a spontaneous increase of contact angle in our system. We propose a mechanism of fatty acid adsorption (after deprotonation) at oil-water interface and the subsequent transfer of these adsorbed molecules to the solid substrate along the drop area. The deprotonation, adsorption and the transfer of the polar molecules depend on salt concentration and aqueous $\mathrm{pH}$, and adsorption is identified as the rate limiting step 
of the autophobing behaviour. AFM investigation of the adsorbed species on the solid phase confirms the mechanistic steps of the phenomenon. We introduce a model, in which the sequence of events are described in differential/time dependent mathematical equations for (amongst others) the adsorbed amounts. The time-scale and extent of wettability alteration of autophobing are captured with two relevant fit parameters.

The novelty of this thesis lies in the systematic approach to unveil and understand adsorption and wetting properties in a very relevant physical system. The combination of experiments and theoretical analysis builds a consistent scenario of electrostatic and ion-specific effect in adsorption leading to macroscopic wettability changes.

\subsection{Future Possibilities}

We have addressed various aspects of adsorption induced wetting properties, and these aspects have been examined in detail, both experimentally and theoretically. Based on the conclusions drawn, it is possible to imagine a number of research questions which will be extremely interesting to investigate.

\subsubsection{Lab-scale Low Salinity Waterflooding}

The wetting experiments described in this thesis shows an oil-wet mineral surface turning into water-wet in the presence of a monovalent salt in the aqueous phase, while a divalent salt solution does not wet the substrate as completely as the former. From an oil-recovery perspective, it is relevant to observe an in-situ change of wettability during a waterflooding process. Hence, a possible direction of investigation is to produce a drop of finite contact angle (using divalent salt solution) on a mineral surface in ambient oil, and subsequently exchange the salt content of the aqueous drop. As the divalent ions from the aqueous drop are removed, and gradually replaced by a monovalent one, the drop should spread slowly, and the contact angle will gradually vanish (implying $\theta<2^{\circ}$ ). The investigation can be carried out at various salt concentration and $\mathrm{pH}$, in order to determine the various thresholds where this transition takes place, i.e., the oil-wet substrate becomes water-wet. 
Since the change in contact angle described in this experiment is quite low $\left(\sim 10^{\circ}\right.$ to $\left.<2^{\circ}\right)$, it is difficult to always perceive such a change. At the same time, for a drop with a low contact angle (such as $10^{\circ}$ ), exchanging the fluid content in the drop is more difficult. Hence, a more realistic goal will be to perform the fluid exchange in the presence of polar organic acid in the oil phase. In the presence of a divalent salt, the autophobing drop's final contact angle is $\sim 70^{\circ}$. Upon changing the salt solution to a monovalent one, the contact angle should decrease down to $<10^{\circ}$ or even less. This is the main effect desirable in a waterflooding process in lab or in a reservoir.

\subsubsection{Wettability Alteration \& Microfluidics}

Waterflooding attempts to release the extra drops of oil attached to the surface. A demonstration of such an effect based on the phenomena described in this thesis can only be achieved in a microfludic channel. It is possible to prepare a microfluidic device where a combination of pore and throat is simulated. It is possible to trap an oil drop at the junction of the pore and throat, and subsequently flush the channel with aqeous salt solution. First allowing high salinity water flow and then switching to a diluted (low salinity) solution should release the trapped oil drop, since the wettability of the surface should change according to the observations made in Chapter 4. Such a demonstration of drop trapping and releasing has been done by the application of electrowetting, or a permanent surface treatment, but has not been attempted with an in-situ chemical wettability alteration.

\subsubsection{Wetting at Reservoir Temperature}

In this work, we have kept the experimental temperature constant at room temperature. However, the temperature of an oil-reservoir is quite different compared to the earth's surface, and depending upon the depth of the reservoir from the surface, can go up to $100-150^{\circ}$. Temperature is one of the main controls of wetting transitions, since increasing (or decreasing) the temperature leads to the critical point of liquid-gas co-existence. The majority of the wetting transition literature has reported a transition from partial to complete wetting upon increasing the temperature. In a possible extension of our experiments, the same set-up could be constructed with the option of increasing the temperature. There will be two counter-acting influences: one of adding salt to the system, which induces finite contact angles; the other, of increased temperature which causes complete wetting in the system. The 
investigation will provide insight about which parameter plays the more important role at reservoir temperature.

\subsubsection{Wetting at Reservoir Pressure}

The km-scale depth of the oil-reservoir also implies higher pressure compared to the earth's surface. Usually, the pressure in an oil-reservoir can be between 10-100 MPa, and wetting experiments at this pressure will be useful to investigate more realistic situations of wettability alteration. 


\section{Thesis Contributions}

\section{Journal Publications}

1. Mugele, F., Bera, B., Cavalli, A., Siretanu, I., Maestro, A., Duits, M.H.G., Cohen-Stuart, M. A., van den Ende, D., Stocker, I., and Collins, I. Ion adsorptioninduced wettability alteration in oil-water-mineral systems. Nature Scientific Reports, 2015, 5, doi:10.1038/srep10519.

2. Cavalli, A., Bera, B., van den Ende, D. and Mugele, F. An analytic model for the electro-wetting properties of oil-water-solid systems. Physical Review E, 2016, Under Review.

3. Bera, B., Kumar, N., Duits, M.H.G., Cohen-Stuart, M. A., van den Ende, D., and Mugele, F. Cationic Hofmeister series of mica wettability in alkanes. Manuscript Under Preparation.

4. Bera, B., Duits, M.H.G., Cohen-Stuart, M. A., van den Ende, D., and Mugele, F. Surfactant Induced Autophobing. Soft Matter, 2016, Under Review.

5. Mugele, F., Siretanu, I., Kumar, N., Bera, B., Wang, L., Maestro, A., Duits, M.H.G. and van den Ende, D. Charge control and wettability alteration at solid-liquid interfaces. SPE Journal, 2015, 5, .

\section{Conference Proceedings}

1. Bera, B., Siretanu, I., Maestro, A., Duits, M., Cohen-Stuart, M. A., van den Ende, H., Mugele, F., and Collins, I. Ion-induced wetting transition during low salinity waterflooding. In EAGE 2015 18th European Symposium on Improved Oil Recovery, pages Dresden, 14-16 April, 2015. 


\section{Prior Publications}

1. Gunde, A. C., Bera, B., and Mitra, S. K. Investigation of water and $\mathrm{CO} 2$ (carbon dioxide) flooding using micro-ct (micro-computed tomography) images of berea sandstone core using finite element simulations. Energy, 35(12):5209-5216.

2. Bera, B., Mitra, S. K., and Vick, D. Understanding the micro structure of berea sandstone by the simultaneous use of micro-computed tomography (micro-CT) and focused ion beam-scanning electron microscopy (FIB-SEM). Micron, 42(5):412-418.

3. Bera, B., Gunda, N. S. K., Mitra, S. K., and Vick, D. Characterization of nanometer-scale porosity in reservoir carbonate rock by Focused Ion BeamScanning Electron Microscopy. Microscopy and Microanalysis, 18(1):171-178.

4. Gunda, N. S. K., Bera, B., Karadimitriou, N. K., Mitra, S. K., and Hassanizadeh, S. M. Reservoir-on-a-chip (roc): A new paradigm in reservoir engineering. Lab on a Chip - Miniaturisation for Chemistry and Biology, 11(22):37853792.

5. Bera, B. and Mitra, S. K. Use of micro-CT images to reconstruct porous media for pore network model. In ASME 2010 8th International Conference on Nanochannels, Microchannels, and Minichannels Collocated with 3rd Joint USEuropean Fluids Engineering Summer Meeting, ICNMM2010, pages 1435-1439. 


\section{About the Author}

Bijoyendra Bera was born on 20th of November, 1987 in Jagacha, Howrah, the twin city of the easterly metropolis Calcutta in India. After finishing higher secondary education at South Point High School, Calcutta (the city came to be known as Kolkata recently) in 2005, he went on to pursue a Bachelor of Engineering in Mechanical Engineering at Jadavpur University, Kolkata. Graduation in 2009 saw Bijoy move all the way to University of Alberta, Edmonton, Canada, where he started a research M.Sc. in Applied Physics. The research involved extracting pore network structure in a real porous medium, and re-creating this network in a 3D micromodel (Lab-ona-Chip) to investigate multiphase flow properties experimentally and numerically. The master thesis was completed in Summer 2011.

In Fall of 2011, Bijoy started as a PhD candidate at Physics of Complex Fluids (PCF) Group within MESA+ Institute of Nanotechnology, under the supervision of Prof. Dr. Frieder Mugele and Dr. Michèl H.G. Duits. The research was focussed upon wetting properties of charged substrates in the presence of two other liquid phases. The doctoral research culminates with this disseration. 



\section{Acknowledgements}

Two roads diverged in a wood, and I-

I took the one less traveled by,

And that has made all the difference.

—Robert Frost

Albeit less travelled, this road has been trodden before. And some of the special people, wise of this road, were there for me, making this difficult and different journey a memorable one.

First of all, I would like to thank my PhD supervisor Prof. Frieder Mugele for giving me the opportunity of carrying out this research. Frieder, I learned a lot from you in these years, but what stands out is realizing what it should take to be a scientist. Your door was always open for any question. Thank you for your patience and for having faith in me in the difficult times. As long as I will do scientific work, I will not have a problem keeping an example in mind.

I am grateful to my daily supervisor Dr. Michèl Duits, for a host of reasons. Michèl, you not only told me how research is firmly related to critical reflection, questioning the uncertain results and tireless effort, but always demonstrated these points yourself.

Very special notes of thanks go to Dr. Dirk van den Ende and Prof. Martien Cohen-Stuart. Dirk, on a fine summer morning (as fine as the dutch weather allows, I'm guessing) in 2011, someone decided that I am to be your office-mate for the next four and a half years. Unbeknownst to me, this was a wonderful day in my life. Soon after joining PCF, I realized that many of the answers to the most difficult physics/research related questions are barely a meter away from me. Thank you for listening to me (at least 5 times a day) and guiding me through the steps of so many interesting problems. 
Martien, when you started looking into my projects, suddenly every experiment had a possible explanation and every theoretical interpretation had a direction, had the scope of becoming something new. Thank you so much for the help and your kind guidance! Verder wil ik graag je ook bedanken voor je bereidheid om met mij alle discussies in het Nederlands te houden, hoewel het jou meer moeite en tijd kostte. Danzij deze discussies, kan ik nu al het technische werk gewoon in het Nederlands doen.

This research was made possible with the financial support from British Petroleum plc., and throughout the years, I met many people at the ExploRe meetings and learned a lot. Thank you Ian, Isabella, Pete Salino, Ann, Prof. Stephan Herminghaus, Prof. Martin Brinkmann, Prof. Ralf Seemann, Prof. Susan Stipp.

I am grateful to Prof. Ann Muggeridge, Prof. Daniel Bonn, Prof. Kitty Nijmeijer, Prof. Juriaan Huskens and Prof. Rene van Roij, for agreeing to be a part of my PhD examination committee.

I would like to thank Andrea for having a significant impact in my learning, work-ethic and $\mathrm{PhD}$ proceedings. Grazie mille, Andrea! Chandra, without your help and support at the very beginning of this project, this work would not fly. Armando, thank you for the crucial measurements in ellipsometry and your positive attitude towards the project and research in general. Igor, thank you for always showing me and the other group members, what modern research is all about!

I would like to take this opportunity to thank all the current PCF members, for providing me with a pleasant work-environment, and fun-filled moments. DanielW, thank you for introducing me to Nanolab, cross-channel project and Bedrijfssport futsal. Thanks to Zhantao, Duc, Rudy, Kartikeya, Davood, Stelian, Fei, Martin, Nicolao, Simone, Adithya, Carla, Edoardo, Olena, Lei, Naveen, Chamy, Cunlu, Jorick. Arjen (our special greeting mode on), dankjewel. Je bent een topper en bedankt voor alles, vooral de goede figuren in dit boek. Aram: Ja man!...PCF was nooit saai dankzij grotendeels jou!

And the past PCF colleagues: thank you all! Riëlle, bedankt voor het luisteren van alle mijn vragen en de keurige adviezen. Jolet, ook voor dezelfde. Burak, thanks for the cool and awesome advice always. Words 
of thanks go to: Sissi, Daniel Ebeling, Alberto, Agata, DanielH, Ivo, Jung, DanielH, Omkar, Somnathda, Yan. Mariska, bedankt voor je hulp met de experimenten. Isabel, Edith, Lisette \& Annelies, thank you for your help with all the administrative work.

The month-long summer school at Ecole de Physique, Les Houches was a significant event in my PhD years. I met the 'creme de la creme's of theoretical colloidal physics, a bunch of extremely smart and nice people, from whom I had much to learn. Jolet and Riëlle, Wilco, Claas-Willem, Sander, Vinzenz, Maxime, Matteo Nicoli, Rob, and of course, the professors: Tom Witten, David Quere, Howard Stone and many others.

Teaching Dynamica for three years has been so much more than assisting in a course: it has been a sheer experience of learning (dynamics and dutch). Thank you Michèl for the opportunity, and thank you Arjen, Jorick, Aram. And of course: thanks to all the students!

Thank you Jorrit and Timen for taking the time to be my paranymphs. Jorrit, het was altijd mogelijk om over alles met jou te praten dankzij je open opvatting naar deze onderwerpen. Ik heb echt veel geleert van je enthousiasme over natuurkunde, ruimtekunde en natuurlijk, voetbal. Timen, wij waren vrienden geworden op mijn eerste weekend in Nederland, en ik heb van jou het belang van rust geleert. Bedankt voor alles! Echt alles!

The four and a half years have seen a whirlpool of activities and fun, and many of them outside the professional chores. I want to thank everybody for these moments of conviviality: Matthias, vielen dank! Vera, thanks for helping me out more than a few times with organizations of events and parties and valuable advice on many occasions. Felix, Thanks for many fun-filled activity or beer evenings. Thanks for the opportunity to play with Lokomotive Döbling. Csaba, Sahar, Edo, Paolo, Daniela, Corina, Gijs, Koen, Laura, Wilbert, Bettina, Feb, Andrei, Aura, Andrea Leoncini, Matteo, Milos, Ivana.............the list is unending .......and so are the nice memories. Olga, Peter, thank you for always staying in touch, and having real good interest at your hearts.

Football has always played a very special part in my daily life....and boy...did it play a very important part in these PhD years! Thanks to everybody at zaalvoetbal: all the opponents I got into a fight with...... or the 
people with whom in the end decided not to: Daniel, Cris, Ruben, Frank $M$, Harmen, Roger, Matthias and innumerable others. As for veldvoetbal: Drienerlo people, you were awesome despite the clear fact that I was not very good. So thank you Frank E, Matthijs, Jeroen, Sander, William, Tukker, Felix (for the amazing football and encouranegment on the pitch), Dennis, Sido, Jelle B, Jelle S, Martijn, David D, David R, Jesse, Auke, Darius, Simon, Martin.......how many do I name? From all over the world, people dropped by to be part of this amazing experience. KTBFFH....Thank you for 19 May, 2012! Yes I was right there! And I will always be! If people would ask me if I had any regret over these four years, I would probably say: yes. I could have squeezed in a couple of more hours of football every week.

I would like to thank my parents for their immense and unconditional care, love and support. You have been my building blocks, and have inspired me (from halfway across the world) everyday through your honesty, perseverence, and passion for work. I have learnt from you that every academic degree or even the highest honour is futile, unless one became a good human being.

Mihaela....I started these words of thanks and gratitude with the mention of a special journey.....and this journey would have been nothing without you. Every moment with you has been worth re-living. You have always been there for me. Your love and support literally saw me through these years. Te Iubesc, and with you at my side, we are up for many such special journeys! 

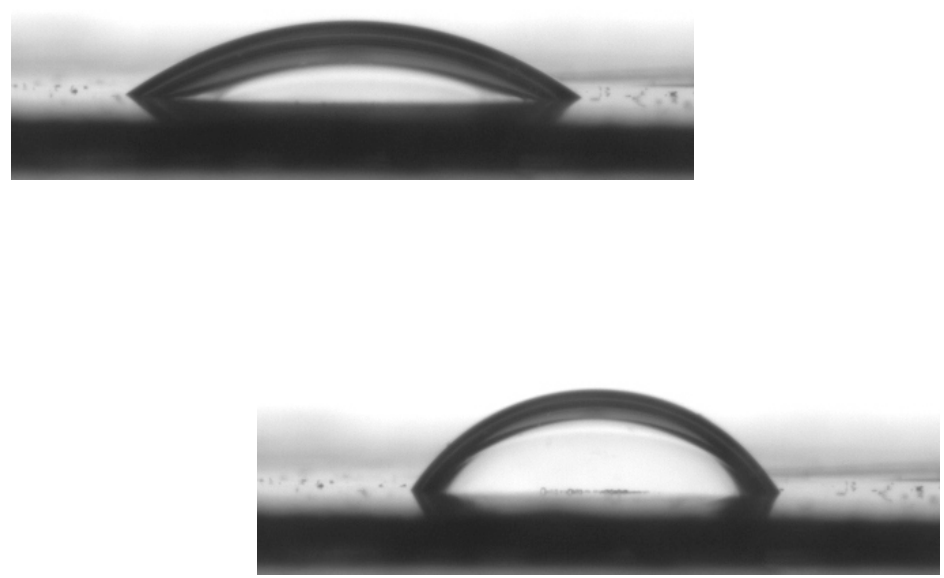

ISBN: 978-90-365-4049-0

\section{UNIVERSITY OF TWENTE.}

